\title{
Theoretical Insights into the Catalytic Hydrogenolysis of Biomass-Derived Cyclic Ethers and Polyols over Metal and Alloy-Promoted Particles in Aqueous Systems
}

\author{
A Dissertation \\ Present to \\ the faculty of the School of Engineering and Applied Science \\ University of Virginia \\ In Partial Fulfillment \\ of the requirement for the Degree of \\ Doctor of Philosophy
}

by

Qiaohua Tan

August

2014 
APPROVAL SHEET

The dissertation

is submitted in partial fulfillment of the requirements

for the degree of

Doctor of Philosophy

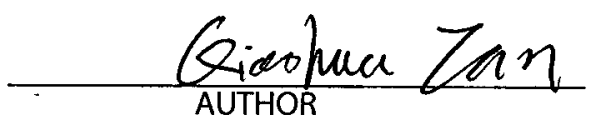

The dissertation has been read and approved by the examining committee:

\begin{tabular}{c} 
Matthew Neurock \\
\hline $\begin{array}{c}\text { Advisor } \\
\text { Robert Davis }\end{array}$ \\
\hline lan Harrison \\
\hline Roseanne Ford \\
\hline Gary Koenig, Jr. \\
\hline
\end{tabular}

Accepted for the School of Engineering and Applied Science:

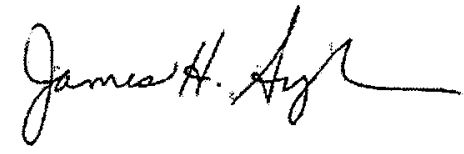

Dean, School of Engineering and Applied Science

August

2014 


\section{Abstract}

The increasing demand for scarce petroleum resources have generated tremendous interest in the development of sustainable strategies that can convert biomass as well as other renewable feed sources into fuels and chemicals. Plant-based sugars and other biomass sources can be deconstructed into polyols and cyclic ethers that contain excess oxygen which must be removed in order to synthesize useful chemical intermediates. This requires rejection of oxygen as either $\mathrm{CO}_{2}, \mathrm{CO}$ or water and the efficient use of hydrogen. In this work, we have used first-principles quantum chemical calculations along with detailed kinetic analyses to examine the fundamental mechanisms that control the selective hydrogenolysis of biomass-derived cyclic ethers and polyols in aqueous media.

Recent experimental efforts have shown that metal alloys comprised of reducible and oxophilic metals such as Re-promoted Rh, Pt, Ir or Pd catalysts selectively activate biomassderived cyclic ethers and polyols such as tetrahydrofurfural alcohol (THFA) at the more substituted carbon to form $\alpha, \omega$-diols with high selectivity and activity. The reaction pathways observed over the non-promoted Rh, Pt, Ir and Pd catalysts are markedly different as they demonstrate high catalytic selectivities to activate the $\mathrm{C}-\mathrm{O}$ bonds of the least-substituted carbon centers which tend to form $\alpha, \beta$-diols. First principle density functional theory (DFT) calculations clearly show that the non-promoted Rh catalyst preferentially activates cyclic ethers and polyols such as THFA and 1,2-propanediol, respectively at the less-substituted carbon center in order to reduce steric repulsion that occurs in activating at the more-substituted carbon center. The direct activation via the metal as was found for $\mathrm{Rh}$ cannot be used to explain the very different catalytic behavior of the Re-promoted Rh system. Our theoretical results together with detailed kinetic experiments strongly suggest that the presence of the very oxophilic Re sites on the surface of 
the Rh-Re catalyst can form strong acid sites in the presence of water. These sites catalyze an acid mechanism that controls the hydrogenolysis of THFA and other cyclic ethers and polyols on the Rh-Re catalysts.

The nature of the active site for hydrogenolysis is still actively debated in the literature. Some studies suggest that Re is partially oxidized and that the active sites may be Re-OH groups while others indicate that the Re is fully reduced. We show that hydroxyl groups as well as water are strongly bound to the Re sites and result in Brønsted acid sites that can catalyze the ring opening of THFA. The unoccupied metallic Re sites on the Rh-Re surface act as Lewis acid sites and can also catalyze the ring opening of THFA. The activation barriers and overall reaction energies for the adsorption, desorption and the dissociation of water are used together with microkinetic models in order to try to predict the relative amounts of the different acid sites on the $\mathrm{Rh}$-Re surface under the reaction condition to elucidate the most plausible active acid sites on the Rh-Re catalyst.

In aqueous solution, solid acids can dissociate to form the hydronium ions, which can influence the activity for reactions that need to occur on the surface of the catalyst. The heterolytic dissociation of the adsorbed water and hydroxyl at the Re sites on Rh-Re surface in water are examined to understand the form of solid Brønsted acid sites on the Rh-Re surface in water by exploring both the dissociated hydronium ions as well as the non-dissociated surface solid acids. Constrained ab initio molecular dynamics simulations are used to quantify the free energy changes for the dissociation reactions, as well as the ring opening reaction of THFA at the acid sites on the Rh-Re surface to further understand the effects of entropy. 


\section{Contents}

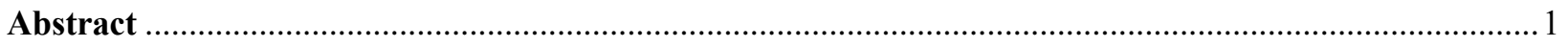

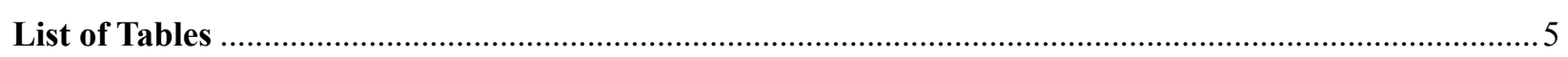

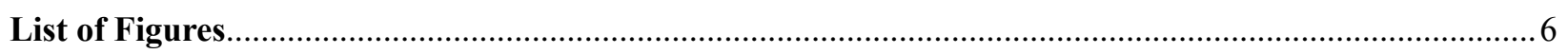

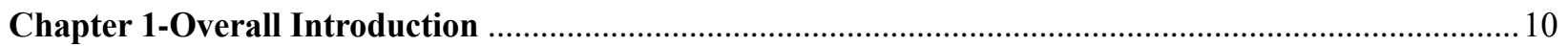

Chapter 2-Hydrogenolysis of 1,2-Propendiol over Rh(111) Surface...................................................... 16

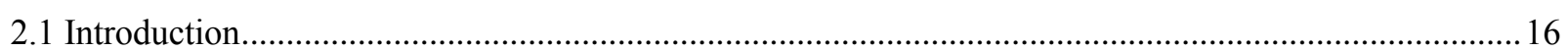

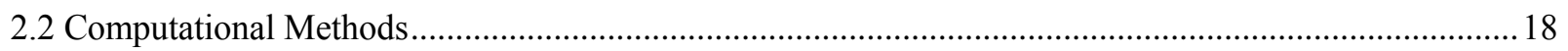

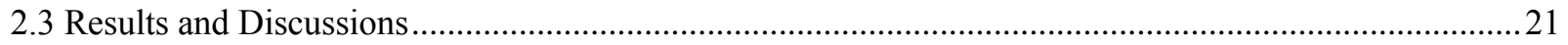

2.3.1 Hydrogenolysis of 1,2-PDO at the less substituted terminal carbon ............................................21

2.3.2 Hydrogenolysis of 1,2-PDO at the more substituted center carbon ...................................................28

2.3.3 Kinetics Analysis of the hydrogenolysis of 1,2-PDO ..................................................................... 33

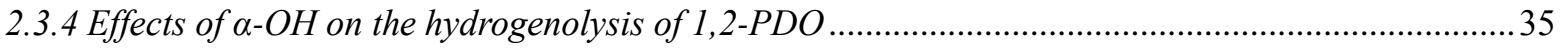

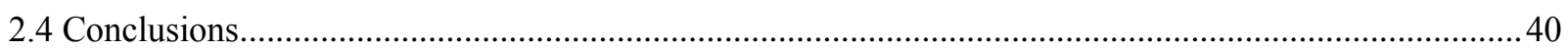

Chapter 3-Selective Hydrogenolysis of Tetrahydrofurfural Alcohol over Rh-Re Catalyst .......................42

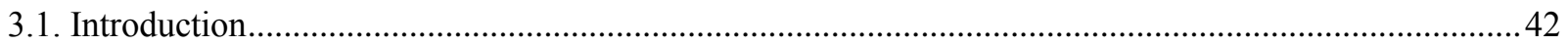

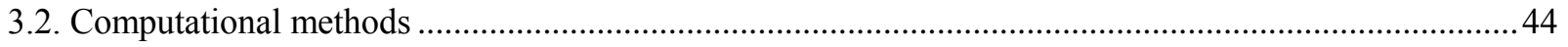

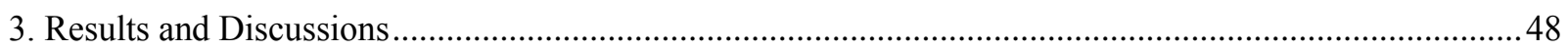

3.1 Water activation on the Rh-Re alloy surface to generate acid site ........................................................ 48

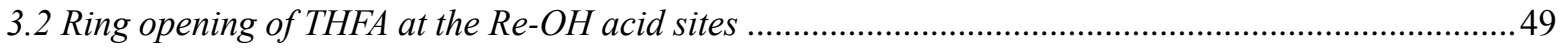

3.3 Hydrogenation of $\delta$-hydroxyvaleraldehyde at the metal sites .............................................................5

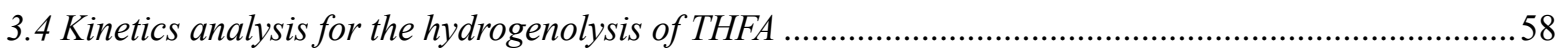

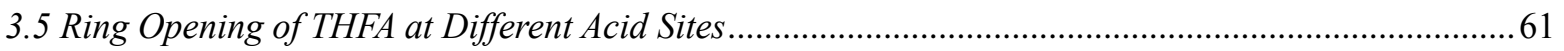

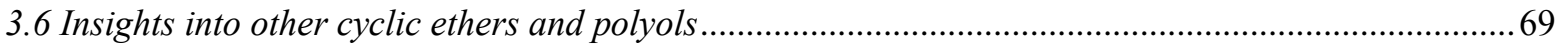

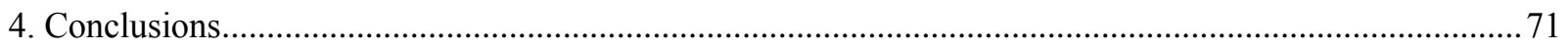

Chapter 4-The Nature of the Active Acid Site: Brønsted vs. Lewis Acid .................................................... 74

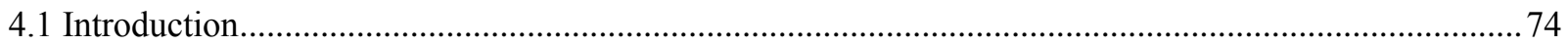

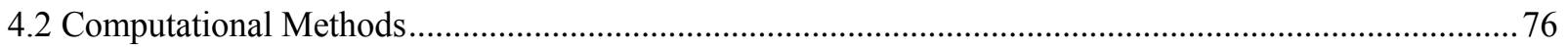

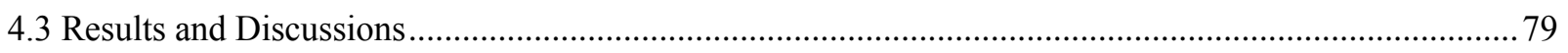

4.3.1 Acid properties of $\mathrm{Re}-\mathrm{OH}_{2}$ and unoccupied Re site on the Rh-Re alloy surface ................................79

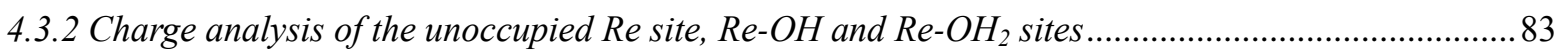

4.3.3 Ring Opening of THFA at the unoccupied Re site and the $\mathrm{Re}-\mathrm{OH}_{2}$ site ........................................... 85

4.3.4 Coverage of the unoccupied Re site, $\mathrm{Re}-\mathrm{OH}_{2}$ and $\mathrm{Re}-\mathrm{OH}$ sites .....................................................92

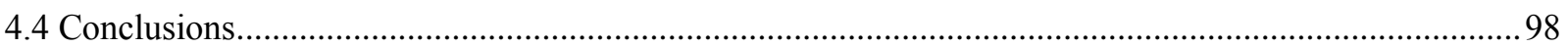

Chapter 5-Entropy Effects on Dissociation of Water and Hydroxyl, and Ring Opening of THFA over the

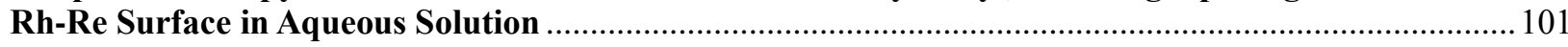

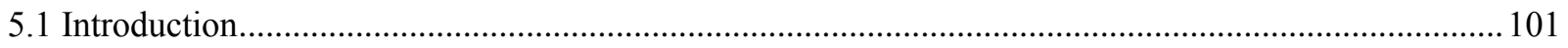




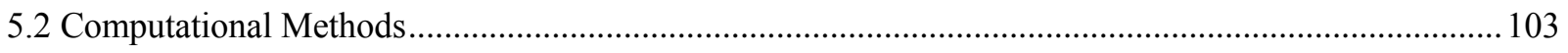

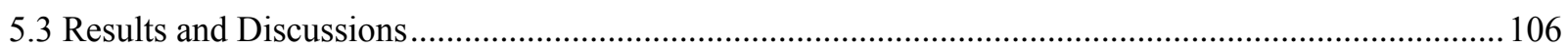

5.3.1 Dissociation of water and hydroxyl at the Re atom on Rh-Re alloy surface ................................... 106

5.3.2 Entropy effects on the dissociation of water and hydroxyl on Rh-Re surface ................................. 110

5.3.3 Dissociation constants for the adsorbed water and hydroxyl on Rh-Re surface............................. 115

5.3.4 Entropy changes for the Ring opening of THFA at the acid sites on Rh-Re surface ........................ 117

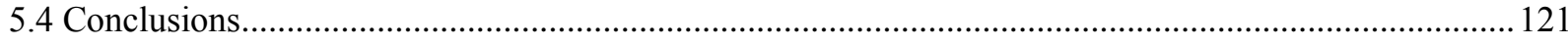

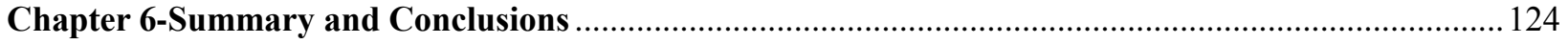

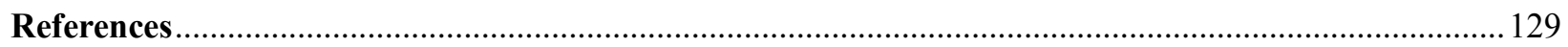




\section{List of Tables}

Table 3.1 DFT-calculated DPE of different Re-OH acid sites on Rh-Re particle, the adsorption energies of THFA and the activation barriers for its ring opening at these different acid sites, together with the binding energies of $\mathrm{O}^{*}$ and $\mathrm{OH}^{*}$ at the Re sites related to the acidity of Re-OH. The units are in $\mathrm{kJ} / \mathrm{mol}$. 62

Table 4.1 DFT calculated DPE of the Brønsted acid Re-OH 2 , Re-OH sites on the Rh-Re particle, ammonia adsorption energies at the $\mathrm{Re}-\mathrm{OH}_{2}, \mathrm{Re}-\mathrm{OH}$ sites and the unoccupied Re sites, and the adsorption energies of $\mathrm{H}_{2} \mathrm{O}, \mathrm{OH}$ and $\mathrm{O}$ at the Re sites on the $\mathrm{Rh}$-Re particle. The units are in $\mathrm{kJ} / \mathrm{mol}$. 80

Table 4.2 Charge analyses of the unoccupied Re atoms, the adsorbed water $\mathrm{Re}-\mathrm{OH}_{2}$ and hydroxyl $\mathrm{Re}-\mathrm{OH}$ with Re located at the terrace, edge and corner sites of the Rh-Re particle ${ }^{*}$...... 83

Table 4.3 Adsorption energies of THFA and activation barriers for its ring opening at Re-OH, $\mathrm{Re}-\mathrm{OH}_{2}$ and unoccupied Re sites. The units are in $\mathrm{kJ} / \mathrm{mol}$. 87

Table 4.4 Free energy changes and equilibrium constants for the reaction steps including the adsorption of $\mathrm{H}_{2} \mathrm{O}$, the activation of $\mathrm{H}_{2} \mathrm{O}^{*}$ to form $\mathrm{OH}^{*}$ and the activation of $\mathrm{OH}^{*}$ to form $\mathrm{O}^{*}$ at the $\mathrm{Re}$ atoms on the Rh-Re surface. The units for $\Delta G$ are in $\mathrm{kJ} / \mathrm{mol}$. 95

Table 4.5 Coverage of the unoccupied Re site, adsorbed water, adsorbed hydroxyl and adsorbed oxygen at the Re atoms on the Rh-Re surface. 96

Table 4.6 Free energy changes calculated in gas phase and in water solvent for the adsorption of $\mathrm{H}_{2} \mathrm{O}$, the activation of $\mathrm{H}_{2} \mathrm{O}^{*}$ to form $\mathrm{OH}^{*}$ and the activation of $\mathrm{OH}^{*}$ to form $\mathrm{O}^{*}$ at the Re atoms on the Rh-Re particle with Re atoms located at the terrace, edge and corner site. The units are in $\mathrm{kJ} / \mathrm{mol}$.

Table 4.7 Equilibrium constants calculated in water solvent for the adsorption of $\mathrm{H}_{2} \mathrm{O}$, the activation of $\mathrm{H}_{2} \mathrm{O}^{*}$ to form $\mathrm{OH}^{*}$ and the activation of $\mathrm{OH}^{*}$ to form $\mathrm{O}^{*}$ at the $\mathrm{Re}$ atoms on the $\mathrm{Rh}-\mathrm{Re}$ particle with Re atoms located at the terrace, edge and corner site. 98

Table 4.8 Coverage of unoccupied Re, adsorbed water, adsorbed hydroxyl and adsorbed oxygen at the different $\mathrm{Re}$ atoms on the $\mathrm{Rh}-\mathrm{Re}$ particle. 98

Table 5.1 Charge analyses of the reactant, transition state and product for the dissociation of the adsorbed water at the Re site on the Rh-Re surface in water solvent. 108

Table 5.2 Charge analyses of the reactant, transition state and product for the dissociation of the adsorbed hydroxyl at the Re site on the Rh-Re surface in water solvent. 110

Table 5.3 Fixed values of the set of constrained reaction coordinates and the calculated mean forces on the constraints calculated by the constrained molecular dynamics simulations on the ring opening of THFA at the Re-OH acid site on the Rh-Re surface in water. The units for the constrained reaction coordinates $\xi$ are in $\AA$, and the units for the mean forces are in $\mathrm{eV} / \AA$.......119 


\section{List of Figures}

Figure 2.1 C-O hydrogenolysis of 1,2-propenediol to form 1-propanol and 2-propanol and the continuing $\mathrm{C}-\mathrm{O}$ hydrogenolysis of 1-propanol and 2-propanol to form propane. 18

Figure 2.2 Possible paths for the hydrogenolysis of 1,2-propenediol at the terminal carbon on $\mathrm{Rh}(111)$ surface. The elementary steps in the red frame are paths that proceed via $\alpha-\mathrm{C}-\mathrm{H}$ elimination intermediates, while the others are paths that proceed via $\beta-\mathrm{C}-\mathrm{H}$ elimination intermediates. The activation energies and reaction energies for steps are listed in the form of $\Delta E_{a c t} / \Delta E_{r x n}$ in the unit of $\mathrm{kJ} / \mathrm{mol}$. The * represent sites on the molecule that are bound to the metal surface. The steps in red represent $\mathrm{C}-\mathrm{H}$ or $\mathrm{O}-\mathrm{H}$ activation steps whereas the steps in blue involve $\mathrm{C}-\mathrm{O}$ activation.

Figure 2.3 DFT calculated reactant, transition state and product structures for the $\mathrm{O}-\mathrm{H}$ bond and the first $\mathrm{C}-\mathrm{H}$ bond activation of 1,2-PDO at the terminal carbon. 23

Figure 2.4 DFT calculated reactant, transition state and product structures for the $\mathrm{O}-\mathrm{H}$ activation (A), C-H activation (B) and $\mathrm{C}-\mathrm{O}$ activation $(\mathrm{C})$ of the $\mathrm{CH}_{3} \mathrm{CH}(\mathrm{OH}) \mathrm{CH}^{*} \mathrm{OH}$ intermediate and the $\mathrm{C}-\mathrm{O}$ activation of the aldehyde intermediate $\mathrm{CH}_{3} \mathrm{CH}(\mathrm{OH}) \mathrm{CH}^{*}=\mathrm{O}^{*}$ (D). 25

Figure 2.5 DFT-calculated reaction energy profiles for the different paths involved in the activation of the $\mathrm{C}-\mathrm{O}$ bond of 1,2-PDO at the terminal carbon through different intermediates formed via $\mathrm{C}-\mathrm{H}$ and $\mathrm{O}-\mathrm{H}$ activation. 27

Figure 2.6 Possible paths for the hydrogenolysis of 1,2-PDO at the center carbon on $\mathrm{Rh}(111)$ surface. The activation energies and reaction energies for steps are listed in the form of $\Delta E_{a c t} / \Delta E_{r x n}$ in the unit of $\mathrm{kJ} / \mathrm{mol}$. The * represent sites on the molecule that are bound to the metal surface. The steps in red represent $\mathrm{C}-\mathrm{H}$ or $\mathrm{O}-\mathrm{H}$ activation steps whereas the steps in blue involve $\mathrm{C}-\mathrm{O}$ activation. 29

Figure 2.7 DFT calculated reactant, transition state and product structures for the $\mathrm{O}-\mathrm{H}$ and $\mathrm{C}-\mathrm{H}$ activation of 1,2-PDO at the center carbon......

Figure 2.8 DFT calculated reactant, transition state and product structures for the $\mathrm{C}$ - $\mathrm{H}$ activation of the alkoxy intermediate $\mathrm{CH}_{3} \mathrm{CH}\left(\mathrm{O}^{*}\right) \mathrm{CH}_{2} \mathrm{OH}(\mathrm{A}), \mathrm{C}-\mathrm{O}$ activation of the ketone intermediate $\mathrm{CH}_{3} \mathrm{C}^{*}=\mathrm{O} * \mathrm{CH}_{2} \mathrm{OH}(\mathrm{B}), \mathrm{H}$ addition of the ketone intermediate $\mathrm{CH}_{3} \mathrm{C}^{*}=\mathrm{O}^{*} \mathrm{CH}_{2} \mathrm{OH}(\mathrm{C})$ and the C-O activation of the intermediate $\mathrm{CH}_{3} \mathrm{C} *(\mathrm{OH}) \mathrm{CH}_{2} \mathrm{OH}(\mathrm{D})$. 31

Figure 2.9 Energy diagrams for the $\mathrm{C}-\mathrm{O}$ breaking of the ketone intermediate $\mathrm{CH}_{3} \mathrm{C}^{*}=\mathrm{O} * \mathrm{CH}_{2} \mathrm{OH}$ through the direct path (blue) and the $\mathrm{H}^{*}$ assisted path (red)...... 32

Figure 2.10 Energy diagrams for the plausible path of C-O breaking of 1,2-PDO at the center carbon. 33

Figure 2.11 Reaction energy diagrams for the plausible paths of the hydrogenolysis of 1,2-PDO at the terminal carbon to form 2-PO (red) and at the center carbon to form 1-PO (black)..... 35

Figure 2.12 Coverage of several important intermediates on the $\mathrm{Rh}(111)$ surface (left) and the 
yield of 2-PO and 1-PO (right) during the kinetics simulation of the hydrogenolysis of 1,2-PDO.

Figure 2.13 DFT calculated reactant and transition state structures for the $\mathrm{O}-\mathrm{H}$ activation of 1,2PDO at the terminal carbon with intramolecular hydrogen bond (left-A), without intramolecular hydrogen bond (left-B) and O-H activation of 1-PO (left-C); O-H activation of 1,2-PDO at the center carbon with intramolecular hydrogen bond (right-A), without intramolecular hydrogen bond (right-B) and $\mathrm{O}-\mathrm{H}$ activation of 2-PO (right-C). 37

Figure 2.14 DFT-calculated reactant and transition state structures for the $\mathrm{C}-\mathrm{H}$ activation of 1,2PDO at the terminal carbon with intramolecular hydrogen bond (left-A), without intramolecular hydrogen bond (left-B) and the $\mathrm{C}-\mathrm{H}$ activation of 1-PO at the terminal carbon (left-C); C-O activation of the intermediate $\mathrm{CH}_{3} \mathrm{CH}(\mathrm{OH}) \mathrm{CH}^{*} \mathrm{OH}$ at the terminal carbon with intramolecular hydrogen bond (right-A), without intramolecular hydrogen bond (right-B) and $\mathrm{C}-\mathrm{O}$ activation of the intermediate $\mathrm{CH}_{3} \mathrm{CH}_{2} \mathrm{CH}^{*} \mathrm{OH}$ (right-C). 39

Figure 3.1 The $4 \mathrm{x} 4 \mathrm{Rh}(111)$ surface with well dispersed $\mathrm{Re}-\mathrm{OH}$ site (left) and the 201-atom cubo-octahedral Rh particle with Re-OH located at different sites (right) used to model the acid sites on Rh-Re catalyst. 47

Figure 3.2 DFT calculated reactant, transition state and product structures for the dissociation of water on the $\mathrm{Rh}-\mathrm{Re}$ and pure $\mathrm{Rh}(111)$ surfaces. 49

Figure 3.3 DFT calculated gas-phase carbenium ion formation energies of MTHF and THFA. 51

Figure 3.4 DFT-calculated reactant and transition state structures for ring opening of THFA and MTHF at the Re-OH acid site on the Rh-Re surface. 52

Figure 3.5 DFT calculated ring opening process of THFA at the Re-OH acid site on Rh-Re surface in aqueous system: A) THFA adsorption at the acid site; B)transition state of the ring opening; C);oxocarbenium ion formation; D) proton shuttling in water; E) regeneration of acid sites; and the charge analyses for the ring opening process...... 53

Figure 3.6 DFT-calculated reactant, transition state and product structures for the hydrogenation of $\delta$-hydroxyvaleraldehyde at the metal sites of Rh-Re surface in aqueous solution through the hydroxylalkyl path: top) first $\mathrm{H}$ addition to the carbonyl oxygen through direct metal insertion mechanism; center) first $\mathrm{H}$ addition to the carbonyl oxygen via PCET mechanism to form a hydroxylalkyl intermediate; bottom) second $\mathrm{H}$ addition to the carbon of the hydroxyalkyl

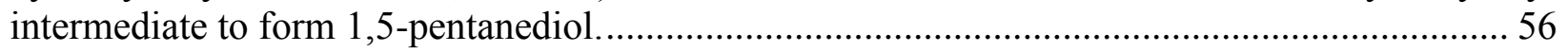

Figure 3.7 DFT-calculated reactant, transition state and product structures for the hydrogenation of $\delta$-hydroxyvaleraldehyde at the metal sites of Rh-Re surface in aqueous solution through the alkoxide path: top) first $\mathrm{H}$ addition to the carbonyl oxygen to form an alkoxide intermediate; bottom) second $\mathrm{H}$ addition to the oxygen of the alkoxide intermediate to form 1,5-pentanediol. 57

Figure 3.8 Potential energy diagrams for the two different paths for the hydrogenation of $\delta$ hydroxyvaleraldehyde at the metal sites on the Rh-Re surface in aqueous solution: (red)

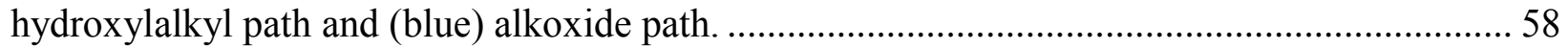


Figure 3.9 Potential energy profiles for the hydrogenolysis of THFA on the bifunctional Rh-Re surface in aqueous solution.

Figure 3.10 Possible elementary steps that occur in the hydrogenolysis process of THFA on RhRe catalyst in aqueous solution. The solid acid sites on Re are denoted as ReOH whereas the metal sites involved in the hydrogenation are listed as *.

Figure 3.11 Different models of the possible acid sites on the Rh-Re catalyst: left) $\mathrm{Re}-\mathrm{OH}$ located at different positions of the Rh cluster (terrace, edge and corner); right) $\mathrm{OH}$ binding 3fold to three adjacent $\mathrm{Re}$ atoms that can be in the $\mathrm{Rh}$ surface or above the Rh surface.

Figure 3.12 DFT-calculated reactant and transition state structures for ring opening of THFA at different Re-OH acid sites on the Rh-Re particle: (A) (111) terrace; (B) edge; (C) corner. 64

Figure 3.13 Correlation of the activation energies for the ring opening of THFA at three different acid sites with $\mathrm{Re}-\mathrm{OH}$ located at the terrace, edge and corner sites of $\mathrm{Rh}_{201}$ particle versus the binding energies of methanol at the vicinal Rh atoms of the acid sites. 65

Figure 3.14 Correlation of the adsorption energies of THFA and activation energies for its ring opening at the three different acid sites $\left(\mathrm{ReOH}, \mathrm{Re}_{3} \mathrm{OH}-\mathrm{IN}\right.$ and $\mathrm{Re}_{3} \mathrm{OH} \_\mathrm{OV}$ ) versus the deprotonation energies of the acid sites.

Figure 3.15 The adsorption energies of THFA and the energy barriers for its ring-opening at different $\mathrm{MOH}$ sites $(\mathrm{M}=\mathrm{Ru}, \mathrm{Mo}, \mathrm{Re}, \mathrm{W})$ embedded in $\mathrm{Ru}, \mathrm{Rh}, \mathrm{Pt}$ and $\mathrm{Au}$ (111) metal surfaces plotted against the $D P E$ of the $\mathrm{MOH}$ sites. 68

Figure 3.16 DFT-calculated reactant and transition state structures for the $\mathrm{C}-\mathrm{O}$ bond activation of 1,2-propanediol at the less substituted terminal carbon (top), at the more substituted center carbon (middle) and the $\mathrm{C}-\mathrm{O}$ bond activation of mono-functional alcohol 2-propanol at the Re$\mathrm{OH}$ acid sites on $\mathrm{Rh}-\mathrm{Re}$ surface (bottom). 70

Figure 3.17 The correlation of the activation barriers for $\mathrm{C}-\mathrm{O}$ bond activation of various cyclic ethers and linear polyols or mono-functional alcohols at the $\mathrm{Re}-\mathrm{OH}$ acid sites on the $\mathrm{Rh}-\mathrm{Re}$ surface versus their corresponding gas phase carbenium ion formation energies. 71

Figure 4.1 Possible acid sites on the Rh-Re alloy surface: the unoccupied Re site, the adsorbed hydroxyl Re-OH and the adsorbed water $\mathrm{Re}-\mathrm{OH}_{2}$ 75

Figure 4.2 The 4x4 Rh(111) surface with well dispersed Re site (left) and the 201-atom cubooctahedral Rh particle with Re located at different sites (right) used to model Rh-Re catalyst. The adsorbed species such as $\mathrm{H}_{2} \mathrm{O}$ and $\mathrm{OH}$ at the Re sites are not shown. 79

Figure 4.3 Linear correlation of the deprotonation energies of $\mathrm{Re}-\mathrm{OH}_{2}$ with $\mathrm{Re}$ atom located at the terrace, edge and corner site of the $\mathrm{Rh}-\mathrm{Re}$ particle versus the difference of the binding energies of $\mathrm{OH}$ and $\mathrm{H}_{2} \mathrm{O}$ at the Re atoms $\left[\Delta E_{a d s}(\mathrm{OH})-\Delta E_{a d s}\left(\mathrm{H}_{2} \mathrm{O}\right)\right]$ 82

Figure 4.4 DFT calculated reactant, transition state and product structures for the ring opening of THFA at the Re-OH, Re-OH 2 and unoccupied Re sites on the Rh-Re surface. 86 
Figure 4.5 DFT calculated reactant and transition state structures for the ring opening of THFA at the terrace, edge and corner $\mathrm{Re}-\mathrm{OH}_{2}$ sites on the $\mathrm{Rh}-\mathrm{Re}$ particle.

Figure 4.6 Charge analyses of the reactant and transition state structures for the ring opening of THFA at the Re site on the Rh-Re alloy surface.

Figure 4.7 DFT calculated reactant and transition state structures for the ring opening of THFA at the terrace, edge and corner Lewis acid Re sites on the Rh-Re particle.

Figure 4.8 Linear correlation of the adsorption energy of THFA and the activation barriers for its ring opening at different $\mathrm{Re}$ sites on the $\mathrm{Rh}$ - $\mathrm{Re}$ particle versus $\mathrm{NH}_{3}$ adsorption energies at these Re sites. 91

Figure 4.9 Reactions that govern the equilibrium between the unoccupied $\mathrm{Re}$ site, $\mathrm{Re}-\mathrm{OH}_{2}, \mathrm{Re}-$ $\mathrm{OH}$ and $\mathrm{Re}-\mathrm{O}$ sites on the Rh-Re surface. 93

Figure 5.1 Static DFT calculated reactant, transition state and product structures for the dissociation of the adsorbed water at the Re site on the Rh-Re surface in water solvent. 107

Figure 5.2 Charge changes from the reactant to the transition state and product for the dissociation of the adsorbed water (left) and hydroxyl (right) at the Re sites on the Rh-Re surface in water solvent. 108

Figure 5.3 Static DFT calculated reactant, transition state and product structures for the dissociation of the adsorbed hydroxyl at the Re site on the Rh-Re surface in water solvent. .... 109

Figure 5.4 Constrained reaction coordinates for the constrained molecular dynamic simulations on the dissociation of the adsorbed water and hydroxyl at the Re site on the Rh-Re surface in water. 111

Figure 5.5 Mean forces on the constraints (left) and the free energy profiles (right) along the reaction coordinate calculated by the constrained AIMD simulations for the dissociation of adsorbed water on the Re site on the Rh-Re surface aqueous solution.

Figure 5.6 Mean forces on the constraints (left) and the free energy profiles (right) along the reaction coordinate calculated by the constrained AIMD simulations for the dissociation of adsorbed hydroxyl intermediates on the Re site on the Rh-Re surface in water.....

Figure 5.7 Constrained reaction coordinates for the constrained molecular dynamics simulations on the ring opening of THFA at the $\mathrm{Re}-\mathrm{OH}$ acid site on the $\mathrm{Rh}-\mathrm{Re}$ surface in water. .118

Figure 5.8 Free energy profiles calculated by the constrained molecular dynamics and thermodynamic integration for the ring opening of THFA at the $\mathrm{Re}-\mathrm{OH}$ acid site on the $\mathrm{Rh}-\mathrm{Re}$ surface in water. 120 


\section{Chapter 1-Overall Introduction}

The use of biomass as a renewable feedstock for the chemical industry is essential to fulfill the long-term demand for carbon-based products. A promising approach for meeting this goal is to convert biomass-derived carbohydrates to platform molecules, which in turn serve as building blocks for commercially-valuable end products. To provide guidance towards the selection of target biorenewable chemicals, the US Department of Energy (DOE) released the DOE 'Top 10' report ${ }^{[1]}$ in 2004 , which outlined a list of potential species including polyols deemed as the most promising platform molecules for a bio-refinery. Dumesic et al. ${ }^{[2,3]}$ expanded on this notion and suggested an array of biologically-derived platform chemicals such as 2-pyrones offering unique opportunities for the conversion of renewable carbohydrates to families of commodity chemicals used by society. We're interested in understanding the catalytic processes involved in converting various biomass-derived platform chemicals into different value-added chemicals and thus establishing a beginning foundation of key intermediates that can used in the production to a wide range of other chemical intermediates.

The most commonly studied biomass feedstocks are plant-based sugars that can produce various polyols and cyclic ethers that contain significant amounts of oxygen which make them unsuitable for fuels and undesirable as chemical intermediates. ${ }^{[4-20]}$ The production of valueadded chemicals from these feeds therefore requires catalytic processes that can selectively remove oxygen either as $\mathrm{CO}_{2}, \mathrm{CO}$ or as water. Catalytic deoxygenation can be accomplished by various different reactions including decarboxylation, decarbonylation, dehydration and $\mathrm{C}-\mathrm{O}$ hydrogenolysis. Of these reactions, selective $\mathrm{C}-\mathrm{O}$ hydrogenolysis over heterogeneous catalysts represents an important class of reactions for the production of high value chemicals without carbon loss. Supported metal catalysts are often used to carry out C-O hydrogenolysis. For 
example, glycerol hydrogenolysis to form a mixture of diols has been studied over various supported-metal catalysts including $\mathrm{Cu}, \mathrm{Ru}, \mathrm{Rh}, \mathrm{Pt}$ and $\mathrm{Pd} .{ }^{[21-50]}$ Recent experimental work suggests that Rh, Pt, and Ir catalysts promoted with Re or Mo display not only high activity, but also high selectivity in the hydrogenolysis of cyclic ethers such as tetrahydrofurfural alcohol (THFA) and polyols such as glycerol and 1,2,6-hexanetriol to their corresponding $\alpha, \omega$-diols. ${ }^{[51-}$ ${ }^{74]}$ Also direct hydrogenolysis of plant-based sugars and sugar polyols such as sorbitol and xylitol on Re promoted Ir catalysts can yield the corresponding $\alpha, \omega$-diol or even n-alkanes with high activity and selectivity. ${ }^{[75,76]}$

Very different reaction patterns are observed, however, in the hydrogenolysis of these cyclic ethers and polyols over non-promoted metal catalysts. Take THFA as an example, over nonpromoted Rh catalyst, about 68\% 1,2-pentanediol and 18\% 1,5-pentanediol are formed, while over Re-promoted Rh catalyst, nearly $94 \%$ 1,5-pentanediol is formed with essentially no formation of 1,2-pentanediol. ${ }^{[51]}$ Despite the growing body of experimental work, the understanding of the fundamental reaction mechanisms that govern the rate and selectivity of CO bond scission reactions of cyclic ethers and polyols over both non-promoted and Re-promoted metal catalysts is still poorly understood. Little is known about the promotional role of Re or the mechanisms for the hydrogenolysis of cyclic ethers and polyols over the non-promoted metal catalysts.

In addition to the unique changes that occur upon alloying with oxophilic promoters, the actual structure and make-up of cyclic ethers and polyols can also significantly influence the hydrogenolysis of cyclic ethers and polyols. A detailed analysis of the hydrogenolysis of various cyclic ethers and polyols carried out over the supported Rh-Re catalysts indicates hydroxyl groups that sit $\alpha$ to the $\mathrm{C}-\mathrm{O}$ bond that is activated can significantly influence the selectivity and 
activity for hydrogenolysis. ${ }^{[51,72]}$ Cyclic ethers such as THFA and polyols such as 1,2propanediol, glycerol and 1,2,6-hexanetriol with $\alpha-\mathrm{OH}$ demonstrate high reactivity and high selectivity over the $\mathrm{C}-\mathrm{O}$ bonds with more substituted carbon sites, while without $\alpha-\mathrm{OH}$ substituent cyclic ethers such as methyl-tetrahydrofuran (MTHF) and polyols such as 1,5pentanediol shows 30 fold decrease in hydrogenolysis activity as well as a marked difference in product selectivity. ${ }^{[51,72]}$ Still, how the $\alpha-\mathrm{OH}$ influences the reactivity of the cyclic ethers and polyols is unknown.

In order to elucidate the nature of the active sites and the mechanisms that control hydrogenolysis, we have carried out a first principle quantum chemical calculations to examine in detail the effects the base metal, the influence of the second metal and oxophilic promoter, the specific structure and composition of the active site, as well as the role of the cyclic ether and polyol reactants on the catalytic reactivity and selectivity.

Unlike the petroleum-based reactions that typically occur in gas phase or in hydrocarbon solvents, the conversions of biomass-based feedstocks are often carried out in aqueous media. ${ }^{[4,}$ 5, 20] Protic solvents can play an important role in carrying reactions by stabilizing polar or charged transition states and can in some cases co-catalyze reactions by allowing for proton transfer. For example, in the hydrogenolysis of THFA over the Rh-Re, the rate of reaction is very high if the reaction is carried out in aqueous solution and negligible if the reaction is carried out in 1,4-dioxane. ${ }^{[63]}$ Thus, to understand the fundamental mechanisms of the catalytic conversion of biomass-based feedstocks in aqueous solutions, both the enthalpic as well as the entropic changes that result due to the presence of solution have to be considered. The entropy effects can be just as important as the enthalpic effects as they require significant changes to local structure and solvent environment which can require the re-organization of the solvent molecules. As such 
entropic effects become important, the changes in the elementary steps that can occur can alter the overall potential energy surface and our understanding of which elementary steps control the rate. Ab initio molecular dynamics simulations more accurately treat the change in free energy than the traditional DFT methods and can be used to more carefully assess the influence of the reaction environment. ${ }^{[77-83]}$ These simulations, however, are significantly more computationally intensive. In this work, we use the more rigorous ab initio molecular dynamics methods to examine the effects of temperature and entropy on the catalytic reactions that occur in solution and at the solvent/metal interfaces in the hydrogenolysis of cyclic ethers and polyols over Rh-Re catalysts. As far as we know, there are no reported studies in the literature on the effects of entropy for reactions that occur at the solvent/metal interfaces.

The mechanisms involved in the selective hydrogenolysis of the model cyclic ether THFA over non-promoted model Rh surfaces were examined in my Master's thesis. ${ }^{[84]}$ Chapter 2 extends this work on ethers to polyols and examines C-O hydrogenolysis of 1,2-propanediol over non-promoted Rh surface. More specifically we examine the possible paths for the C-O bond activation of 1,2-propanediol at both the less substituted terminal carbon to form 2-propanol and the more substituted center carbon to form 1-propanol. Kinetics analyses are performed to understand which step governs the reactivity and selectivity in the hydrogenolysis process. Structural effects on the reactivity of polyols are explored by comparing 1,2-propanediol with the corresponding mono-functional alcohols 1-propanol and 2-propanol.

Chapter 3 explores the mechanisms for the hydrogenolysis of THFA and other cyclic ethers and polyols on the Re-promoted Rh catalyst. As the selectivity for the hydrogenolysis of THFA over Rh-Re catalyst is not consistent with the metal catalyzed mechanism, experimental results along with our previous DFT calculations suggest that acid sites are formed on the Rh-Re 
catalyst ${ }^{[72,73]}$ and participate in a bifunctional mechanism. Herein, we examine in detail the plausible paths and mechanisms for the hydrogenolysis of THFA at the bifunctional Rh-Re catalyst including the ring opening step at the acid sites and the hydrogenation steps at the metal sites. Different acid sites on the Rh-Re catalyst are investigated for the ring opening of THFA to understand the influence of the acidity of the acid sites. The structural effects of the $\alpha-\mathrm{C}-\mathrm{OH}$ in the molecule of THFA and the solvent effects on the reactivity of THFA are also illustrated. The paths and mechanism are then extended to other cyclic ethers and polyols. In addition, we extend the efforts to a range of other bimetallic surfaces/particles comprised of a reducible metal $(\mathrm{Rh}$, $\mathrm{Pt}$, Ir and Pd) to carry out the hydrogenation reactions and an oxophilic promoter metal ( $\mathrm{Re}, \mathrm{Mo}$, $\mathrm{W}$ and $\mathrm{Ru}$ ) to carry out acid catalyzed steps.

Chapter 4 more thoroughly examines the possible acid sites on the Rh-Re surfaces as there are different interpretations of the XANES and EXAFS results which result in different suggestions as to the nature of the active Re site. Detailed studies by Davis et al. ${ }^{[85]}$ suggest that the $\mathrm{Re}$ is partially oxidized and that the active sites may be $\mathrm{Re}-\mathrm{OH}$ groups which might suggest the presence of Brønsted acid sites. Studies by Miller et al. ${ }^{[73]}$ report very similar XANES results but suggest that the Re is fully reduced which might suggest that the Re act as Lewis acid sites or that adsorbed water at these sites behaves as a Brønsted acid. We show that hydroxyl groups as well as water are strongly bound to the Re sites and result in Brønsted acid sites that can catalyze the ring opening of THFA. The unoccupied metallic Re sites on the Rh-Re surface act as Lewis acid sites and can also catalyze the ring opening of THFA. The activation barriers and overall reaction energies for the adsorption, desorption and the dissociation of water are used together with microkinetic models in order to try to predict the relative amounts of the different acid sites on the Rh-Re surface under the reaction condition to elucidate the most plausible active 
acid sites on the Rh-Re catalyst.

In Chapter 5, we continue to study the heterolytic dissociation of the adsorbed water and hydroxyl at the Re sites on Rh-Re surface in water to understand the form of solid Brønsted acid site on the Rh-Re surface in water by exploring both the dissociated hydronium ion as well as the non-dissociated surface solid acids. Constrained molecular dynamics simulations are used to quantify the free energy changes for the dissociation reactions, as well as the ring opening reaction of THFA at the acid sites on the Rh-Re surface to further understand the effects of entropy. 


\section{Chapter 2-Hydrogenolysis of 1,2-Propendiol over Rh(111) Surface}

\subsection{Introduction}

The mechanisms involved in the selective hydrogenolysis of cyclic ethers such as THFA over non-promoted Rh catalysts were examined in detail in my Master's thesis. ${ }^{[84]}$ The results showed that the activation of the C-O bond of THFA at the less-substituted secondary and moresubstituted tertiary carbon centers occur after all the hydrogens on the carbon that is activated are eliminated. The more substituted carbons present steric constraints that result in higher $\mathrm{C}-\mathrm{H}$ activation barriers than those on the less-substituted carbon sites. The ring opening of THFA therefore was found to be favored at the less substituted carbon resulting in higher selectivity of 1,2-pentanediol over that of 1,5-pentanediol which agrees with experimental results. ${ }^{[51]}$ In this work, we extend these ideas to $\mathrm{C}-\mathrm{O}$ hydrogenolysis of polyols over non-promoted $\mathrm{Rh}$ surfaces before examining the promoter effects of Re or Mo in the next chapter.

In general, the hydroxyl groups in sugar polyols can be characterized by the degree of substitution of the carbon at which they are bound thus resulting in terminal primary $\mathrm{OH}$ groups and central secondary or tertiary $\mathrm{OH}$ groups. The most studied polyol is glycerol, which contains two $-\mathrm{OHs}$ at the two symmetric terminal carbons and one $-\mathrm{OH}$ at the center carbon. The hydrogenolysis of glycerol has been examined over various monometallic catalysts. ${ }^{[21-50]}$ The main products that form on the monometallic systems tend to result from the activation of the terminal C-O bond to form 1,2-propanediol (1,2-PDO), the scission of the C-C bond to form ethylene glycol and minor amounts of central bond $\mathrm{C}-\mathrm{O}$ scission to form 1,3-propanediol and other over-hydrogenolysis products. Most of these studies focused on controlling the selectivity between 1,2-propanediol and ethylene glycol by altering the solution $\mathrm{pH}$ or by adding cocatalysts. There has been somewhat less focus on altering the selectivity between the breaking of 
the terminal $-\mathrm{OH}$ and the center $-\mathrm{OH}$. While there is a higher selectivity of 1,2-propanediol than 1,3-propanediol in the hydrogneolysis of glycerol, we are not able to tell which of C-OH bonds is more reactive since there're two times more terminal $\mathrm{OH}$ groups, and due to the loss of both diol products via subsequent hydrogenolysis paths that lead to other by-products. Even though glycerol hydrogenolysis has been studied in detail in the literature, the mechanism for C-O bond hydrogenolysis remains in question. Thus, here, we use 1,2-propenediol instead of glycerol as a simple model polyol compound with only one terminal $\mathrm{C}-\mathrm{OH}$ and one center $\mathrm{C}-\mathrm{OH}$ in the structure to explore the mechanism of the selective hydrogenolysis of polyols on Rh catalysts. 1,2-PDO is also a valuable biomass-based platform chemical as it can be used to form 1- and 2propanol which are also valuable chemical intermediates used in solvents, printing inks and in the production of n-propyl acetate. ${ }^{[86,87]}$ Compared with the mono-functional alcohols, there is the extra $-\mathrm{OH}$ groups that resides alpha to the $\mathrm{C}-\mathrm{OH}$ bond that is activated that may influence the C-O hydrogenolysis activity. Herein we compare the hydrogenolysis of 1,2-propanediol with its corresponding mono-functional alcohol 1-propanol and 2-propanol to explore the effects of the $\alpha-\mathrm{OH}$.

The hydrogenolysis of 1,2-PDO at the terminal carbon results in the formation of 2propanol (2-PO) whereas the hydrogenolysis at the central carbon atom results in the formation of 1-propanol (1-PO) as shown in Figure 2.1. The subsequent C-O hydrogenolysis of the resulting propanol products ultimately lead to formation of propane. Various studies indicate that dehydrogenation of polyols and monofunctional alcohols tends to precede C-O bond hydrogenolysis over metal catalysts. ${ }^{[88]}$ Glycerol hydrogenolysis is thought to proceed via the formation of glyceraldehyde which can further react to form 1,2 and 1,3 propanediol as well as other intermediates on $\mathrm{Ru}$ and $\mathrm{Pt}$ catalysts. ${ }^{[39]}$ In the theoretical calculations for the 
hydrogenolysis of 1,2-PDO, we examined all the possible pathways for the hydrogenolysis of 1,2-PDO through different intermediates that result from the different $\mathrm{H}$ elimination steps in order to establish the most plausible paths for the hydrogenolysis of 1,2-PDO at the terminal as well as the secondary -C-OH sites.

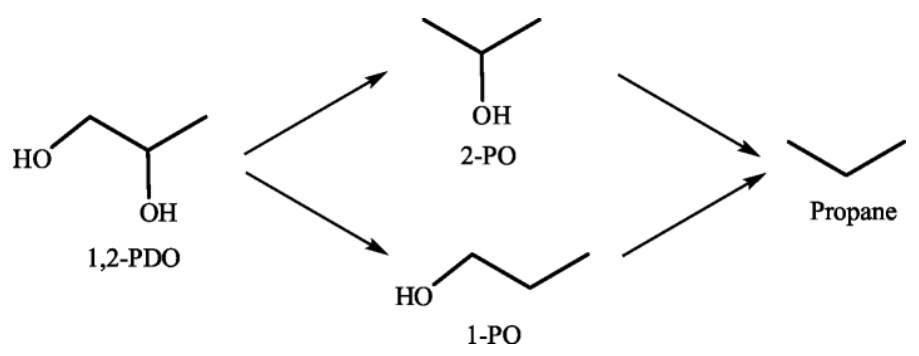

Figure 2.1 C-O hydrogenolysis of 1,2-propenediol to form 1-propanol and 2-propanol and the continuing $\mathrm{C}-\mathrm{O}$ hydrogenolysis of 1-propanol and 2-propanol to form propane.

\subsection{Computational Methods}

All of the calculations reported herein were carried out using periodic plane-wave gradientcorrected density functional theory methods as implemented in the Vienna ab initio Simulation Package (VASP). ${ }^{[89-92]}$ The Perdew-Wang 91 (PW91) ${ }^{[93]}$ form of generalized gradient approximation (GGA) exchange correlation functional was used to provide the non-local gradient-corrections to exchange and correlation energies. Wave functions were constructed by using projector augmented wave potentials (PAW) ${ }^{[94,95]}$ within a cutoff energy of $396.0 \mathrm{eV}$.

The close-packed $3 \times 3 \mathrm{Rh}$ (111) surface with four metal layers and $15 \AA$ of vacuum separating slabs in the z-direction was used for all of the calculations reported herein. The top two metal layers were allowed to relax in the calculations whereas the bottom two layers were held fixed to their bulk position. The electronic energies were converged to within $10^{-6} \mathrm{eV}$. The geometric structures for all of the calculations were optimized until the forces were converged to 
within $0.05 \mathrm{eV} / \AA$ using a $6 \times 6 \times 1$ Monkhorst-Pack ${ }^{[96]} \mathrm{k}$-point mesh to sample the first Brillouin zone. The convergence criteria of $0.05 \mathrm{eV} / \AA$ was verified by further optimizing a set of structures presented in this paper to a convergence criteria of $0.01 \mathrm{eV} / \AA \AA$. This tighter convergence criteria resulted in changes to the overall energy of $<0.02 \mathrm{eV}$, which is believed to be smaller than the intrinsic error in the method. The energies of the adsorbates in vacuum were calculated spin-polarized using an $18 \times 18 \times 18 \AA$ unit cell with the $\Gamma$ only k-point mesh.

The nudged elastic band (NEB) method ${ }^{[97]}$ was used to find reaction coordinates by linearly interpolating 16 images between the initial and final states and then minimizing the energy of the string of images until the forces perpendicular to the reaction path converged within $0.25 \mathrm{eV} / \AA$. Reliable estimations of the transition state and reaction mode were obtained from the NEB results and used in the dimer method to isolate the transition states and establish the lowest energy path. ${ }^{[98]}$ The dimer methods use two images that close to the transition state to form a 'dimer' structure. The transition state was located by moving the 'dimer' uphill along the potential energy surface. The 'dimer' is also rotated along this path in order to follow the lowest curvature mode of the potential energy. The dimer calculations were carried out until the forces perpendicular to the reaction mode were converged to within $0.05 \mathrm{eV} / \AA$ at a $6 \times 6 \times 1 \mathrm{k}$-point mesh to sample the first Brillouin zone.

Charge analysis was performed by using the QUAMBO ${ }^{[99-102]}$ method, which transforms the wave-functions of the VASP calculations into spatially localized non-orthogonal quasi-atomic orbitals. This transformation provides information about the atomic orbitals of the system, and permits a Löwdin charge and bond order analysis. ${ }^{[103,104]}$

The binding energies of the surface species were calculated as:

$$
\Delta E_{a d s}=E_{M+i}-E_{M}-E_{i}
$$


where $E_{M+i}$ is the total energy of the metal surface with the adsorbate $i$ bound to the metal, $E_{M}$ is the total energy of the bare metal surface and $E_{i}$ is the total energy of the adsorbate in gas phase.

The activation barriers and reaction energies were calculated as:

$$
\begin{gathered}
\Delta E_{A C T}=E_{T S}-E_{R} \\
\Delta E_{r x n}=E_{P}-E_{R}
\end{gathered}
$$

respectively where $E_{T S}$ is the total energy of the transition state, $E_{R}$ is the total energy of the reactant state and $E_{P}$ is the total energy of the product state. For $E_{R}$ and $E_{P}$, if more than one species are adsorbed on the surface, they were calculated as:

$$
E_{R} \text { or } E_{P}=E_{M+1}+E_{M+2}+\cdots E_{M+n}-(\mathrm{n}-1) E_{M}
$$

where $E_{M+1}$ is the total energy of the metal surface with adsorbed species 1 and similarly $E_{M+2}$ and $E_{M+n}$ are the total energies of the metal surface with adsorbed species 2 and species $n$ with the assumption that there is no interaction between species on the metal surface.

In the kinetics analyses, the reaction rate for each surface reaction elementary step is calculated using transition state theory, with the rate given by

$$
r_{i}=v_{i} \exp \left(-\frac{\Delta E_{i}}{R T}\right)
$$

where $v_{i}$ is the pre-exponential factor, $R$ is the gas constant, $T$ is the temperature, and $\Delta E_{i}$ is the activation barrier for the elementary reaction $i$. The pre-exponential factor $v_{i}$ were chosen herein to be standard statistical mechanical estimates for surface processes. For unimolecular surface reactions and (immobile) bimolecular reactions, the pre-exponential factors were estimated to be $10^{13} \cdot[105,106]$ The pre-exponential factors for desorption were also estimated from statistical mechanics to be on the order of $10^{13} \cdot{ }^{[105,106]}$ According to the literature, the simulated results were not significantly affected by changes in the pre-exponential factors. ${ }^{[105]}$

The adsorption rates were calculated by ${ }^{[107-109]}$ 


$$
r_{a d s, i}=s_{0} \cdot P_{i} \cdot A_{S} \cdot\left(2 \pi \cdot m_{i} \cdot k T\right)^{-0.5} \cdot \exp \left(-\frac{\Delta E_{i}}{R T}\right)
$$

where $s_{0}$ is the sticking coefficient, $P_{i}$ is the partial pressure of species $i, A_{S}$ is the area of one surface site, and $m_{i}$ is the molecular weight of species $i$. Here, we take the sticking coefficient of hydrogen to be 0.1 based on the literature ${ }^{[110]}$ and the sticking coefficient of 1,2-PDO to be 1.0 for simplification as there's no experimental results reported. The influence of the choice of the sticking coefficients in the range of $0 \sim 1.0$ is small according to the literature. ${ }^{[105]}$ The kinetics analyses herein were performed at the temperature of $300 \mathrm{~K}$ with the $\mathrm{H}_{2}$ pressure of 1.0 bar. 1,2PDO is taken as gas phase reactant with a gas phase pressure of 1.0 bar.

The ordinary differential equations in the kinetics analyses were solved by Matlab ${ }^{[111]}$ using ode45 function.

\subsection{Results and Discussions}

\subsubsection{Hydrogenolysis of 1,2-PDO at the less substituted terminal carbon}

The possible overall paths for the hydrogenolysis of 1,2-PDO at the terminal carbon shown in Figure 2.2 involve $\mathrm{C}-\mathrm{H}$ activation as well as $\mathrm{O}-\mathrm{H}$ bond activation steps, and ultimately $\mathrm{C}-\mathrm{O}$ activation. The sequence of steps can be different and as such result in the formation of various intermediates. First principle DFT calculations were used to calculate the overall reaction energies as well as the activation barriers for each of these steps and to probe the most favorable paths for the hydrogenolysis of 1,2-PDO at the terminal carbon as well as the central carbon. 


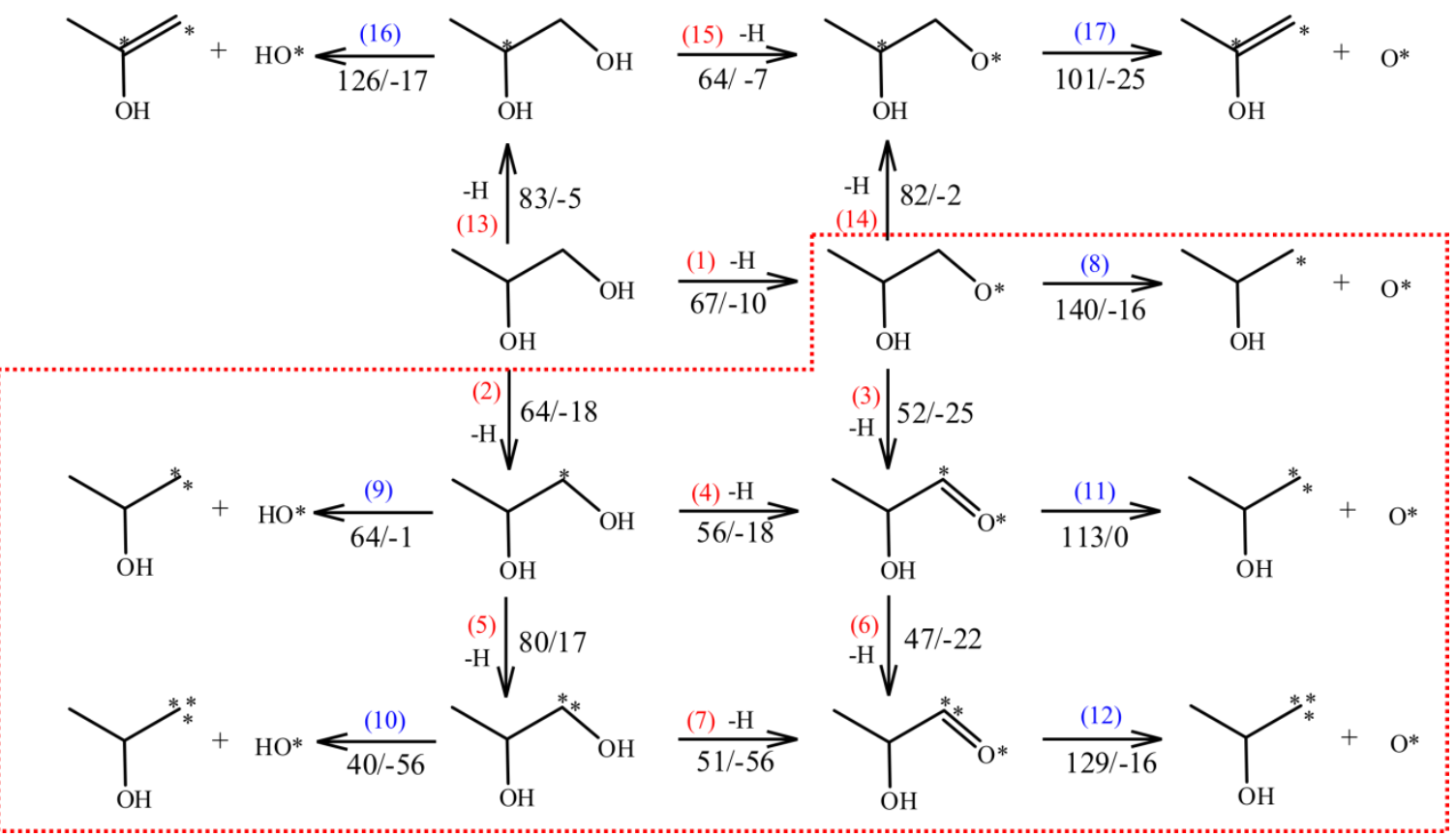

Figure 2.2 Possible paths for the hydrogenolysis of 1,2-propenediol at the terminal carbon on $\mathrm{Rh}(111)$ surface. The elementary steps in the red frame are paths that proceed via $\alpha-\mathrm{C}-\mathrm{H}$ elimination intermediates, while the others are paths that proceed via $\beta-\mathrm{C}-\mathrm{H}$ elimination intermediates. The activation energies and reaction energies for steps are listed in the form of $\Delta E_{a c t} / \Delta E_{r x n}$ in the unit of $\mathrm{kJ} / \mathrm{mol}$. The * represent sites on the molecule that are bound to the metal surface. The steps in red represent $\mathrm{C}-\mathrm{H}$ or $\mathrm{O}-\mathrm{H}$ activation steps whereas the steps in blue involve $\mathrm{C}-\mathrm{O}$ activation.

As shown in Figure 2.3, 1,2-PDO adsorbs on the $\mathrm{Rh}(111)$ surface through the two hydroxyl oxygen atop two adjacent Rh atoms with one Rh-O (at the terminal carbon) distance of $2.38 \AA$ and another Rh-O (at the central carbon) distance of $2.53 \AA$. A hydrogen bond is also formed between the two hydroxyl group with $\mathrm{OH} \cdots \mathrm{OH}$ distance of $2.18 \AA$. The adsorption energy is calculated to be $-45 \mathrm{~kJ} / \mathrm{mol}$, which is slightly higher than the reported adsorption energy of ethanol on $\mathrm{Rh}(111)$ surface through only one hydroxyl group $(38 \mathrm{~kJ} / \mathrm{mol}){ }^{[26]}$ The adsorbed 1,2PDO can then activate the $\mathrm{O}-\mathrm{H}$ bond at the terminal carbon to form an alkoxy intermediate $\mathrm{CH}_{3} \mathrm{CH}(\mathrm{OH}) \mathrm{CH}_{2} \mathrm{O}$ * The $\mathrm{O}-\mathrm{H}$ activation of 1,2-PDO at the terminal carbon proceeds through the typical metal insertion mechanism via a classic 3-center $\mathrm{Rh}-\mathrm{O}-\mathrm{H}$ transition state which has an 
Rh-O distance of $2.14 \AA$, an O-H distance of $1.46 \AA$ and an $\mathrm{Rh}-\mathrm{H}$ distance of $2.08 \AA$ as shown in Figure 2.3. It is noted that in the transition state, the hydrogen bond between the central secondary $\mathrm{OH}$ group and the oxygen of the terminal $\mathrm{OH}$ group is still intact with an $\mathrm{OH} \cdots \mathrm{OH}$ distance of $2.15 \AA$. The activation energy is calculated to be $67 \mathrm{~kJ} / \mathrm{mol}$, which is the same with the reported energy barrier for the $\mathrm{O}-\mathrm{H}$ activation of glycerol at the terminal carbon on $\mathrm{Rh}(111)$ surface $(67 \mathrm{~kJ} / \mathrm{mol}){ }^{[26]}$

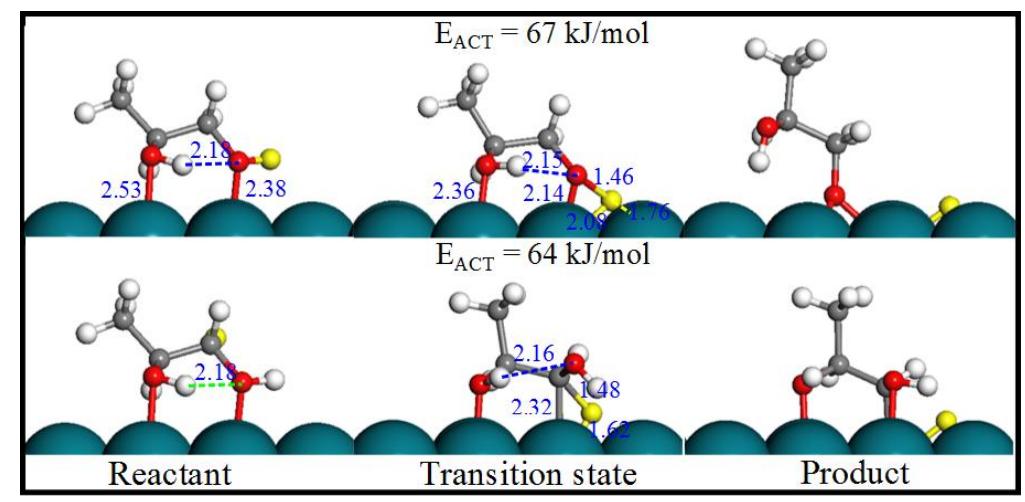

Figure 2.3 DFT calculated reactant, transition state and product structures for the $\mathrm{O}-\mathrm{H}$ bond and the first $\mathrm{C}-\mathrm{H}$ bond activation of 1,2-PDO at the terminal carbon.

1,2-PDO can also activate one of the $\mathrm{C}-\mathrm{H}$ bonds at the terminal carbon to form the $\mathrm{CH}_{3} \mathrm{CH}(\mathrm{OH}) \mathrm{CH}^{*}-\mathrm{OH}$ intermediate as shown in Figure 2.3. This step also proceeds through a metal insertion with a 3-center Rh-C-H triangle state structure with an Rh-C distance of $2.32 \AA$, a C-H distance of $1.48 \AA$ and an Rh-H distance of $1.62 \AA$. The activation energy was calculated to be $64 \mathrm{~kJ} / \mathrm{mol}$, which is somewhat lower than the reported $\mathrm{C}-\mathrm{H}$ activation of glycerol at the terminal carbon $(74 \mathrm{~kJ} / \mathrm{mol}) .{ }^{[26]}$ The higher energy barrier for the $\mathrm{C}-\mathrm{H}$ bond activation of glycerol at the terminal carbon is likely due to the change in the intramolecular hydrogen bond between the central $-\mathrm{OH}$ group and the terminal $-\mathrm{OH}$ group. The energy barrier for the $\mathrm{C}-\mathrm{H}$ activation is slightly lower than the $\mathrm{O}-\mathrm{H}$ activation at the terminal carbon of 1,2-PDO, indicating 
the $\mathrm{C}-\mathrm{H}$ bond activation is slightly more favorable than the $\mathrm{O}-\mathrm{H}$ activation.

After the $\mathrm{CH}_{3} \mathrm{CH}(\mathrm{OH}) \mathrm{CH}^{*}-\mathrm{OH}$ hydroxyalkyl intermediate is formed, it can continue to react via the activation of the $\mathrm{O}-\mathrm{H}$ bond at the terminal carbon to form the aldehyde intermediate $\mathrm{CH}_{3} \mathrm{CH}(\mathrm{OH}) \mathrm{CH}^{*}=\mathrm{O}^{*}$ with the energy barrier of $56 \mathrm{~kJ} / \mathrm{mol}$. The hydroxyalkyl intermediate can also react by activating the second $\mathrm{C}-\mathrm{H}$ bond to form the $\mathrm{CH}_{3} \mathrm{CH}(\mathrm{OH}) \mathrm{C}^{* *}-\mathrm{OH}$ intermediate that binds at the bridge site of the $\mathrm{Rh}(111)$ surface with the energy barrier of $80 \mathrm{~kJ} / \mathrm{mol}$. The barrier is $\sim 16 \mathrm{~kJ} / \mathrm{mol}$ higher than the activation of the first $\mathrm{C}-\mathrm{H}$ bond $(64 \mathrm{~kJ} / \mathrm{mol})$. This trend is different from what we found in the $\mathrm{C}-\mathrm{H}$ activation of cyclic ether tetrahydrofurfural alcohol (THFA) where the activation of the second $\mathrm{C}-\mathrm{H}$ bond at the secondary ether carbon was found to be easier than that of the first $\mathrm{C}-\mathrm{H}$ bond. ${ }^{[84]}$ The results shown in Figure 2.4 indicate that upon breaking the second $\mathrm{C}-\mathrm{H}$ bond, the terminal carbon moves from the atop site to the bridge site on the $\mathrm{Rh}(111)$ surface, which forces the $-\mathrm{OH}$ at the center carbon to change from the bound state to the unbound state. From the reactant to the transition state, the $\mathrm{Rh}-\mathrm{O}$ distance for the $-\mathrm{OH}$ at the center carbon increases from $2.26 \AA$ to $2.43 \AA$, indicating the weaker binding of the $-\mathrm{OH}$ at the center carbon in the transition state. As well, the distance for the intramolecular hydrogen bond between the two -OHs increases from $2.27 \AA$ in the reactant to $3.03 \AA$ in the transition state, indicating weaker hydrogen bond in the transition state. It is the weaker binding of the $-\mathrm{OH}$ at the center carbon to the metal surface and the weaker intramolecular hydrogen bond in the transition state that results in the higher energy barrier for the second $\mathrm{C}-\mathrm{H}$ bond activation at the terminal carbon. 


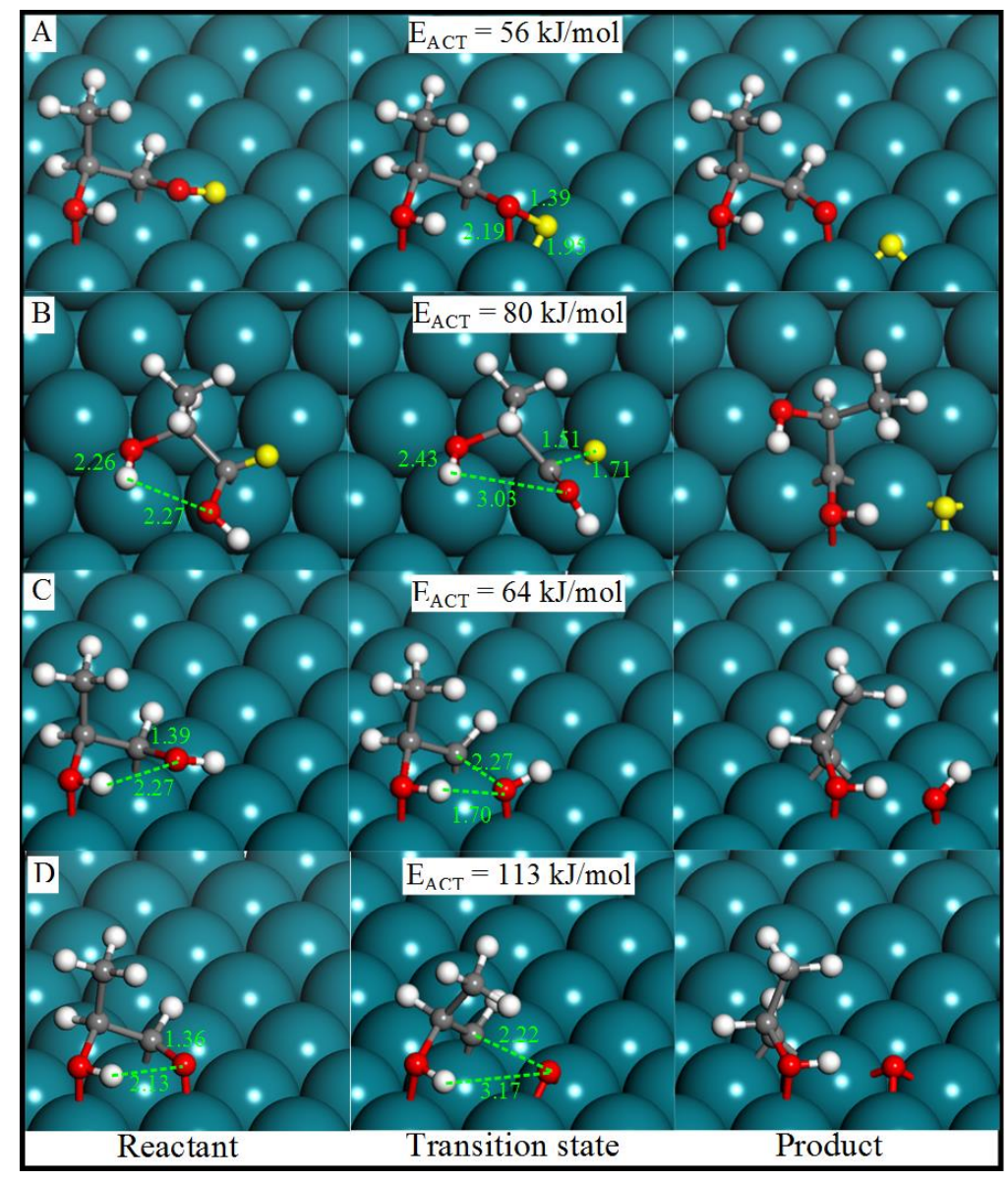

Figure 2.4 DFT calculated reactant, transition state and product structures for the $\mathrm{O}-\mathrm{H}$ activation (A), C-H activation (B) and $\mathrm{C}-\mathrm{O}$ activation (C) of the $\mathrm{CH}_{3} \mathrm{CH}(\mathrm{OH}) \mathrm{CH}^{*} \mathrm{OH}$ intermediate and the $\mathrm{C}-\mathrm{O}$ activation of the aldehyde intermediate $\mathrm{CH}_{3} \mathrm{CH}(\mathrm{OH}) \mathrm{CH}^{*}=\mathrm{O}^{*}$ (D).

Besides continuing to activate the $\mathrm{O}-\mathrm{H}$ bond or the secondary $\mathrm{C}-\mathrm{H}$ bond, the $\mathrm{CH}_{3} \mathrm{CH}(\mathrm{OH}) \mathrm{CH}^{*}-\mathrm{OH}$ intermediate can also directly activate the $\mathrm{C}-\mathrm{O}$ bond to form the $\mathrm{CH}_{3} \mathrm{CH}(\mathrm{OH}) \mathrm{CH}^{* *}$ and $\mathrm{OH}^{*}$ intermediates as shown in Figure 2.4. We can see that in the transition state, the $\mathrm{C}-\mathrm{O}$ bond elongates from $1.39 \AA$ to $2.27 \AA$ and the distance for the intramolecular hydrogen bond between the two -OHs decreases from $2.27 \AA$ to $1.70 \AA$, indicating stronger hydrogen bond in the transition state. The energy barrier is calculated to be only $64 \mathrm{~kJ} / \mathrm{mol}$. Comparison of these $\mathrm{O}-\mathrm{H}, \mathrm{C}-\mathrm{H}$ and $\mathrm{C}-\mathrm{O}$ activation steps of $\mathrm{CH}_{3} \mathrm{CH}(\mathrm{OH}) \mathrm{CH}^{*}$ $\mathrm{OH}$ shows that the $\mathrm{O}-\mathrm{H}$ activation to form the aldehyde intermediate $\mathrm{CH}_{3} \mathrm{CH}(\mathrm{OH}) \mathrm{CH}^{*}=\mathrm{O}^{*}$ is the 
most favorable with the lowest energy barrier. The C-O double bond activation of the aldehyde intermediate $\mathrm{CH}_{3} \mathrm{CH}(\mathrm{OH}) \mathrm{CH}^{*}=\mathrm{O}^{*}$ at the terminal carbon to form $\mathrm{CH}_{3} \mathrm{CH}(\mathrm{OH}) \mathrm{CH}^{* *}$ and $\mathrm{O}^{*}$ however is difficult with the energy barrier of $113 \mathrm{~kJ} / \mathrm{mol}$ as shown in Figure 2.4 .

The reaction energy diagrams for the different paths involved in the activation of the terminal $\mathrm{C}-\mathrm{O}$ bond of 1,2-PDO result in different intermediates depending upon whether the reaction proceeds via C-H, O-H or C-OH bond activation as is shown in Figure 2.5. The most favorable path for the activation of 1,2 PDO involves the activation of the terminal $\mathrm{C}-\mathrm{H}$ bond to form the $\mathrm{CH}_{3} \mathrm{CH}(\mathrm{OH})-\mathrm{CH}^{*} \mathrm{OH}$ hydroxyalkyl intermediate which can subsequently undergo $\mathrm{C}-\mathrm{OH}, \mathrm{O}-\mathrm{H}$ or C-H bond scission. The results shown in Figure 2.5 indicate that the hydroxyalkyl intermediate preferentially reacts via $\mathrm{O}-\mathrm{H}$ scission to form the $\mathrm{CH}_{3} \mathrm{CH}(\mathrm{OH})-\mathrm{CH}^{*}=\mathrm{O}^{*}$ aldehyde $(56 \mathrm{~kJ} / \mathrm{mol})$. While the aldehyde readily forms, it cannot undergo the subsequent $\mathrm{C}-\mathrm{O}$ activation to result in the hydrogenolysis of the $\mathrm{C}-\mathrm{O}$. It instead reacts via $\mathrm{C}-\mathrm{OH}$ activation to form the deoxygenated $\mathrm{CH}_{3} \mathrm{CH}(\mathrm{OH})-\mathrm{CH}^{* *}$ along with $* \mathrm{OH}$ with a barrier of only $64 \mathrm{~kJ} / \mathrm{mol}$, which is only $8 \mathrm{~kJ} / \mathrm{mol}$ higher than that to form the aldehyde. The hydroxyalkyl intermediate can also undergo a second $\mathrm{C}-\mathrm{H}$ activation step. The barrier for this step however is rather prohibitive at $80 \mathrm{~kJ} / \mathrm{mol}$. This is rather different than what we found in the hydrogenolysis of cyclic ether THFA on $\mathrm{Rh}(111)$ surface which proceeds via two subsequent $\mathrm{C}-\mathrm{H}$ activation steps prior to $\mathrm{C}-\mathrm{O}$ scission. ${ }^{[84]}$ 

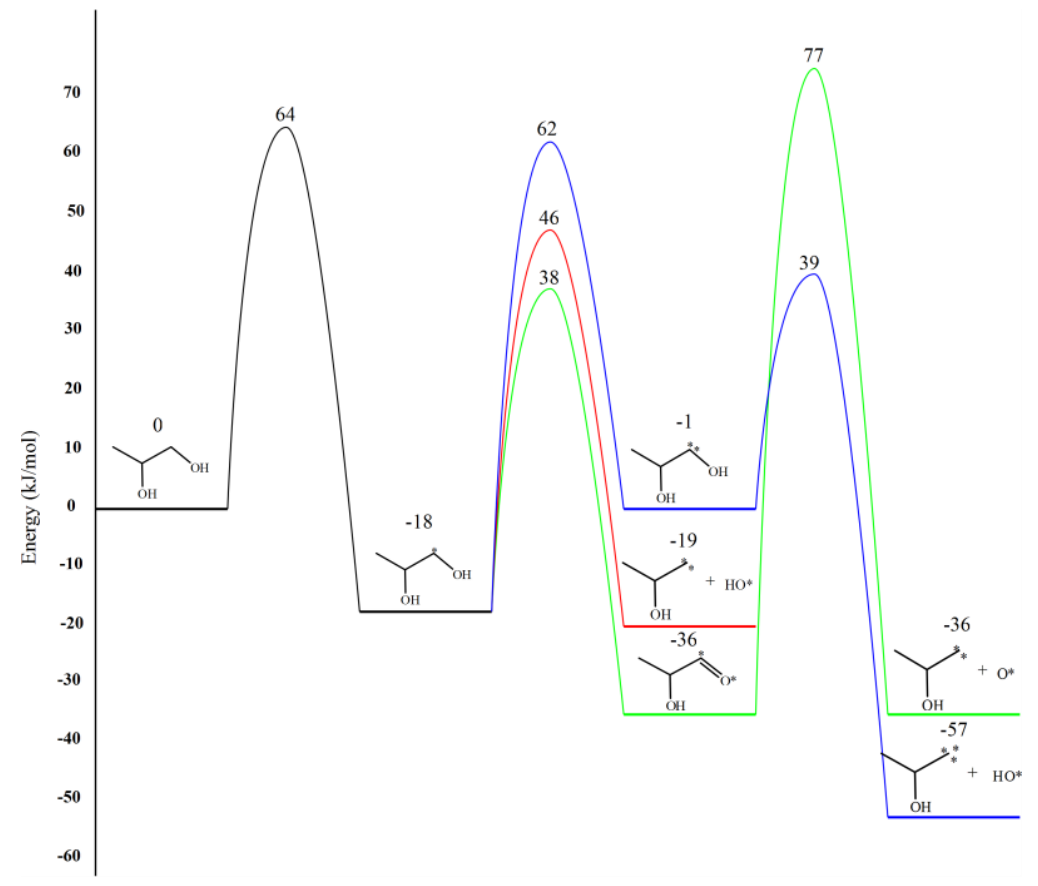

Figure 2.5 DFT-calculated reaction energy profiles for the different paths involved in the activation of the $\mathrm{C}-\mathrm{O}$ bond of 1,2-PDO at the terminal carbon through different intermediates formed via $\mathrm{C}-\mathrm{H}$ and $\mathrm{O}-\mathrm{H}$ activation.

Oxametallacycle intermediates formed via $\beta-\mathrm{C}-\mathrm{H}$ activation have been reported experimentally on $\mathrm{Rh}(111)$ in the decomposition of ethanol. ${ }^{[88]}$ Thus, we also examined the paths of the hydrogenolysis of 1,2-PDO at the terminal carbon on $\mathrm{Rh}(111)$ surface through $\beta-\mathrm{C}-\mathrm{H}$ activation intermediates as shown in Figure 2.2 (Rxn 13-17). It was found that the most favorable path for the $\mathrm{C}-\mathrm{O}$ bond breaking of 1,2-PDO at the terminal carbon through $\beta-\mathrm{C}-\mathrm{H}$ activation intermediates is that 1,2-PDO activates the $\mathrm{C}-\mathrm{H}$ bond at the center carbon to form the intermediate $\mathrm{CH}_{3} \mathrm{C} *(\mathrm{OH}) \mathrm{CH}_{2} \mathrm{OH}$, followed by the subsequent activation of the terminal $\mathrm{O}-\mathrm{H}$ bond to form the oxametallacycle intermediate $\mathrm{CH}_{3} \mathrm{C}^{*}(\mathrm{OH}) \mathrm{CH}_{2} \mathrm{O}^{*}$, which then breaks the C-O bond to form an enol intermediate $\mathrm{CH}_{3} \mathrm{C}^{*}(\mathrm{OH})=\mathrm{CH}_{2}{ }^{*}$ and $\mathrm{O}^{*}$. The activation energy associated with activating the $\beta-\mathrm{C}-\mathrm{H}$ bond at the more substituted center carbon $(83 \mathrm{~kJ} / \mathrm{mol})(R x n 13)$ is higher than activating the $\alpha-\mathrm{C}-\mathrm{H}$ bond (64 kJ/mol) (Rxn 2) at the less substituted terminal carbon. 
The paths associated with the hydrogenolysis of 1,2-PDO through the $\beta-\mathrm{C}-\mathrm{H}$ activation is not as favorable as the paths through $\alpha-\mathrm{C}-\mathrm{H}$ bond activation intermediates as discussed above.

\subsubsection{Hydrogenolysis of 1,2-PDO at the more substituted center carbon}

The reaction paths associated with the hydrogenolysis of 1,2-PDO at the more substituted center carbon were also examined. The results which are reported in Figure 2.6 indicate that 1,2PDO can react either via the activation of the central O-H bond to form the $\mathrm{CH}_{3} \mathrm{CHO}^{*}-\mathrm{CH}_{2} \mathrm{OH}$ alkoxide $(71 \mathrm{~kJ} / \mathrm{mol})$, the central $\mathrm{C}-\mathrm{H}$ bond to form the secondary $\mathrm{CH}_{3} \mathrm{C}^{*} \mathrm{OH}-\mathrm{CH}_{2} \mathrm{OH}$ hydroxyalkyl $(83 \mathrm{~kJ} / \mathrm{mol})$. The activation of the $\mathrm{O}-\mathrm{H}$ bond proceeds over the $\mathrm{Rh}(111)$ surface via metal insertion into the $\mathrm{O}-\mathrm{H}$ bond as shown in Figure 2.7 resulting in a barrier of $71 \mathrm{~kJ} / \mathrm{mol}$, which is slightly higher than that for the activation of the $\mathrm{O}-\mathrm{H}$ bond at the terminal carbon atom (67 kJ/mol). 1,2-PDO can also react via the scission of the $\mathrm{C}-\mathrm{H}$ bond at the central carbon center to form central secondary $\mathrm{CH}_{3} \mathrm{C}^{*}(\mathrm{OH})-\mathrm{CH}_{2} \mathrm{OH}$ hydroxyalkly intermediate shown in Figure 2.7 with a barrier of $83 \mathrm{~kJ} / \mathrm{mol}$, which is $19 \mathrm{~kJ} / \mathrm{mol}$ higher than that required to activate the terminal secondary $\mathrm{C}-\mathrm{H}$ bond at the terminal carbon $(64 \mathrm{~kJ} / \mathrm{mol})$. This higher barrier to activate the $\mathrm{C}-\mathrm{H}$ bond at the more substituted carbon results from steric repulsion between the - $\mathrm{CHOH}$ substituent and vicinal $\mathrm{Rh}$ atoms that result as the active $\mathrm{Rh}$ atom inserts into the $\mathrm{C}-\mathrm{H}$ bond. Similar results which demonstrate the selective activation at the least substituted $\mathrm{C}-\mathrm{H}$ bond have also been reported in the hydrogenolysis of THFA and glycerol. ${ }^{[26,84]}$ 


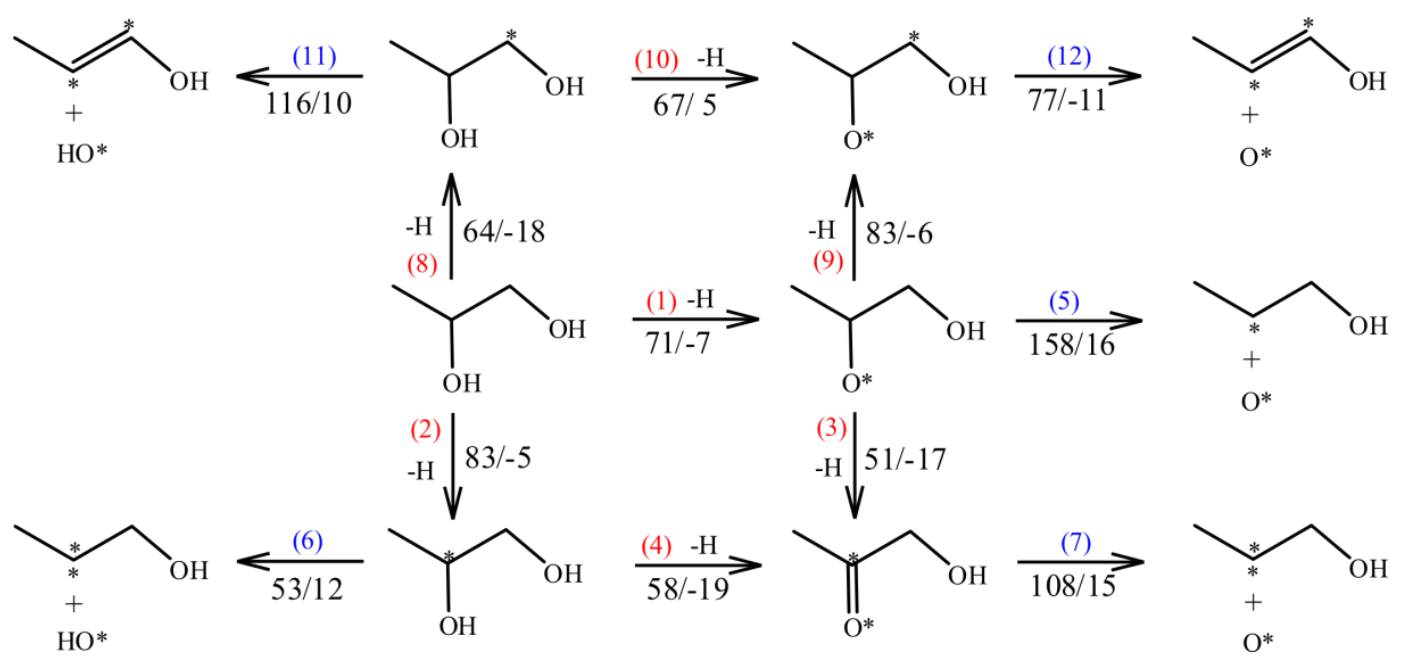

Figure 2.6 Possible paths for the hydrogenolysis of 1,2-PDO at the center carbon on $\mathrm{Rh}(111)$ surface. The activation energies and reaction energies for steps are listed in the form of $\Delta E_{a c t} / \Delta E_{r x n}$ in the unit of $\mathrm{kJ} / \mathrm{mol}$. The * represent sites on the molecule that are bound to the metal surface. The steps in red represent $\mathrm{C}-\mathrm{H}$ or $\mathrm{O}-\mathrm{H}$ activation steps whereas the steps in blue involve $\mathrm{C}-\mathrm{O}$ activation.

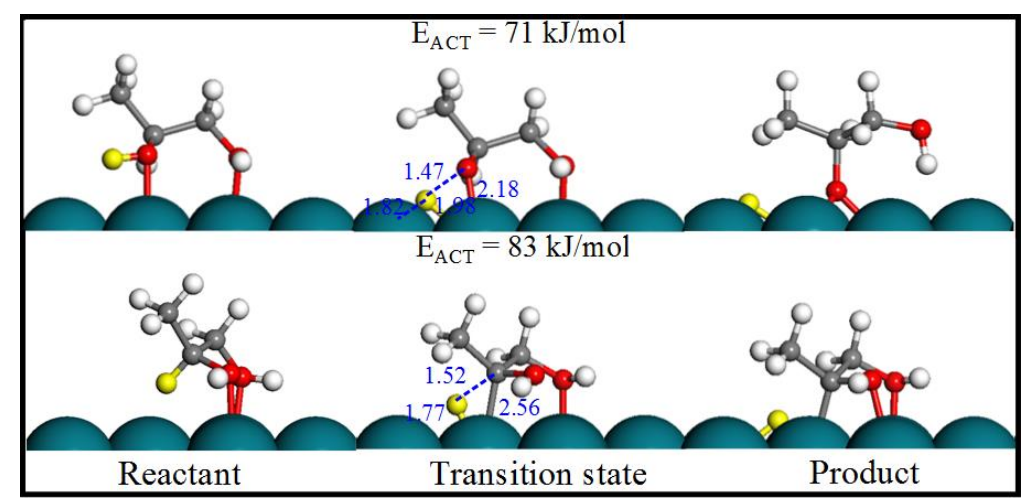

Figure 2.7 DFT calculated reactant, transition state and product structures for the $\mathrm{O}-\mathrm{H}$ and $\mathrm{C}-\mathrm{H}$ activation of 1,2-PDO at the center carbon.

The results in Figure 2.7 show that the activation of the $\mathrm{O}-\mathrm{H}$ bond of at the central carbon of 1,2 PDO is actually more favorable than the activation of the $\mathrm{C}-\mathrm{H}$ bond at this same $\mathrm{C}$ center as the barrier for $\mathrm{O}-\mathrm{H}$ activation is $12 \mathrm{~kJ} / \mathrm{mol}$ lower than that for $\mathrm{C}-\mathrm{H}$ activation. This is characteristically different from the activation of the 1,2 PDO at the terminal carbon which proceeds via $\mathrm{C}-\mathrm{H}$ bond activation. The $\mathrm{CH}_{3} \mathrm{CH}\left(\mathrm{O}^{*}\right)-\mathrm{CH}_{2} \mathrm{OH}$ alkoxide that forms upon the 
secondary $\mathrm{CO}-\mathrm{H}$ activation of 1,2-PDO can either react via the scission of the $\mathrm{C}-\mathrm{O}$ bond to form the $\mathrm{CH}_{3} \mathrm{CH}-\mathrm{CH}_{2} \mathrm{OH}^{*}+\mathrm{O}^{*}$ or via the scission of the secondary $\mathrm{C}-\mathrm{H}$ bond to form the $\mathrm{CH}_{3} \mathrm{C}^{*}=\mathrm{O}^{*}-\mathrm{CH}_{2} \mathrm{OH}$ ketone. The barrier to break the $\mathrm{C}-\mathrm{O}$ bond of the secondary alkoxide was calculated to be very high at $158 \mathrm{~kJ} / \mathrm{mol}$ and as such does not occur. The alkoxide instead preferentially activates its center $\mathrm{C}-\mathrm{H}$ bond with a barrier of $51 \mathrm{~kJ} / \mathrm{mol}$ to form $\mathrm{CH}_{3} \mathrm{C}^{*}=\mathrm{O}^{*}-\mathrm{CH}_{-}$ ${ }_{2} \mathrm{OH}$ ketone as shown in Figure 2.8. This barrier is $\sim 32 \mathrm{~kJ} / \mathrm{mol}$ lower than that for the $\mathrm{C}-\mathrm{H}$ bond activation of 1,2-PDO at the center carbon $(83 \mathrm{~kJ} / \mathrm{mol})$ thus indicating that the $\mathrm{O}-\mathrm{H}$ bond activation at the center carbon facilitates the subsequent $\mathrm{C}-\mathrm{H}$ bond activation.

The subsequent activation of the $\mathrm{C}=\mathrm{O}$ bond of the ketone to form $\mathrm{CH}_{3} \mathrm{C}^{*}-\mathrm{CH}_{2} \mathrm{OH}$ is very difficult with the energy barrier of $108 \mathrm{~kJ} / \mathrm{mol}$, which is similar to the energy barrier for the $\mathrm{C}-\mathrm{O}$ double bond breaking of the aldehyde intermediate shown above $(113 \mathrm{~kJ} / \mathrm{mol})$. The energies reported in Figure 2.8 indicate that ketone can instead react with hydrogen via the H-addition to the $\mathrm{O}^{*}$ to form the $\mathrm{CH}_{3} \mathrm{C}^{*}(\mathrm{OH})-\mathrm{CH}_{2} \mathrm{OH}$ hydroxyalkyl intermediate with a barrier of $77 \mathrm{~kJ} / \mathrm{mol}$. The hydroxyalkyl intermediate can then undergo direct $\mathrm{C}-\mathrm{O}$ bond scission with a much lower activation barrier of $53 \mathrm{~kJ} / \mathrm{mol}$. The energy diagrams for the direct $\mathrm{C}-\mathrm{O}$ double bond activation and the $\mathrm{H}^{*}$ assisted $\mathrm{C}-\mathrm{O}$ double bond activation of the ketone intermediate $\mathrm{CH}_{3} \mathrm{C}^{*}=\mathrm{O}^{*}-\mathrm{CH}_{2} \mathrm{OH}$ were shown in Figure 2.9. We can see the overall energy barrier for the $\mathrm{H}^{*}$ assisted C-O double bond activation path is $77 \mathrm{~kJ} / \mathrm{mol}$, which is much lower than the energy barrier for the direct C-O double bond activation path $(134 \mathrm{~kJ} / \mathrm{mol})$. 


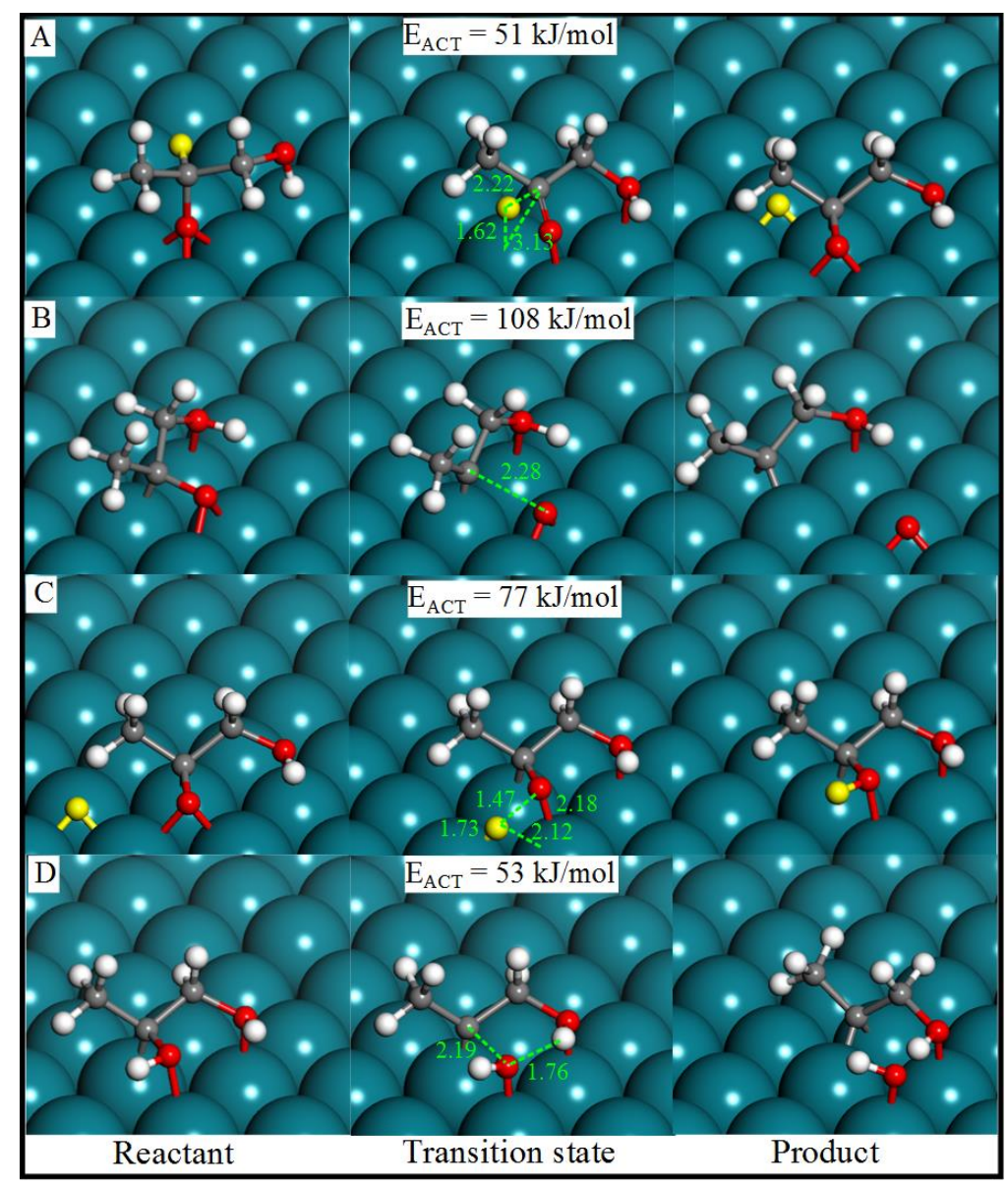

Figure 2.8 DFT calculated reactant, transition state and product structures for the $\mathrm{C}$ - $\mathrm{H}$ activation of the alkoxy intermediate $\mathrm{CH}_{3} \mathrm{CH}\left(\mathrm{O}^{*}\right) \mathrm{CH}_{2} \mathrm{OH}(\mathrm{A}), \mathrm{C}-\mathrm{O}$ activation of the ketone intermediate $\mathrm{CH}_{3} \mathrm{C}^{*}=\mathrm{O} * \mathrm{CH}_{2} \mathrm{OH}(\mathrm{B}), \mathrm{H}$ addition of the ketone intermediate $\mathrm{CH}_{3} \mathrm{C}^{*}=\mathrm{O}^{*} \mathrm{CH}_{2} \mathrm{OH}(\mathrm{C})$ and the $\mathrm{C}-\mathrm{O}$ activation of the intermediate $\mathrm{CH}_{3} \mathrm{C}^{*}(\mathrm{OH}) \mathrm{CH}_{2} \mathrm{OH}(\mathrm{D})$.

The hydrogenolysis of the central $\mathrm{C}-\mathrm{OH}$ can also proceed via the initial activation of the $\beta$ $\mathrm{C}-\mathrm{H}$ bond which resides at the terminal carbon as shown in Figure 2.6 (Rxn 8-12). As was discussed earlier, the activation of the terminal $\mathrm{C}-\mathrm{H}$ bond of $1,2-\mathrm{PDO}(64 \mathrm{~kJ} / \mathrm{mol})$ is $19 \mathrm{~kJ} / \mathrm{mol}$ lower than that for the activation of the central $\mathrm{C}-\mathrm{H}$ bond due to the avoidance of the steric repulsion of the terminal $\mathrm{C}-\mathrm{H}$ bond. The barrier to activate the terminal $\mathrm{C}-\mathrm{H}$ bond is also slightly $(7 \mathrm{~kJ} / \mathrm{mol}$ ) lower than that for activating the $\mathrm{O}-\mathrm{H}$ bond at the central secondary carbon. As such 1,2-PDO can activate the $\beta$ (terminal)-C-H bond to form the terminal hydroxyalkyl intermediate 
$\left(\mathrm{CH}_{3} \mathrm{CH}(\mathrm{OH}) \mathrm{CH}^{*} \mathrm{OH}\right)$ and can subsequently activate the central $\mathrm{O}-\mathrm{H}$ bond at the secondary carbon to form the $\mathrm{CH}_{3} \mathrm{CH}\left(\mathrm{O}^{*}\right) \mathrm{CH}^{*} \mathrm{OH}$ oxametallacycle intermediate. The $\mathrm{C}-\mathrm{O}$ bond of the oxametallacyle can subsequently rupture to form a $\mathrm{CH}_{3} \mathrm{CH}^{*}=\mathrm{C} * \mathrm{HOH}$ enol intermediate and $\mathrm{O}^{*}$ with a barrier of $77 \mathrm{~kJ} / \mathrm{mol}$. The $\mathrm{CH}_{3} \mathrm{CH}^{*}=\mathrm{C}^{*} \mathrm{HOH}$ enol and $\mathrm{O}^{*}$ can then be hydrogenated to form the products, 1-PO and water. $\mathrm{O}^{*}$, however, is strongly bound to $\mathrm{Rh}(111)$ and as such is difficult to hydrogenate as it must overcome a barrier of $119 \mathrm{~kJ} / \mathrm{mol}$. As such, the paths for the hydrogenolysis of 1,2-PDO at the center carbon through the activation of the $\beta-\mathrm{C}-\mathrm{H}$ bond is not a favorable path.

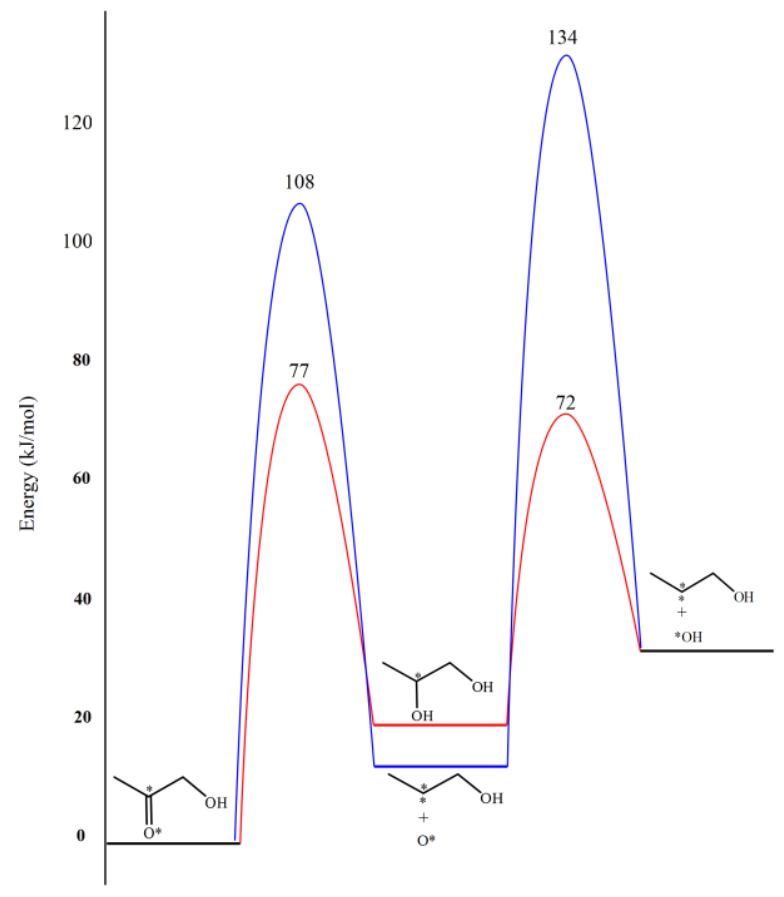

Figure 2.9 Energy diagrams for the $\mathrm{C}-\mathrm{O}$ breaking of the ketone intermediate $\mathrm{CH}_{3} \mathrm{C}^{*}=\mathrm{O}^{*} \mathrm{CH}_{2} \mathrm{OH}$ through the direct path (blue) and the $\mathrm{H}^{*}$ assisted path (red).

We thus concluded that the $\mathrm{C}-\mathrm{O}$ breaking of 1,2-PDO at the center carbon proceeds via the formation of the $\mathrm{CH}_{3} \mathrm{C}^{*}(\mathrm{OH})-\mathrm{CH}_{2} \mathrm{OH}$ hydroxyalkyl intermediate, which cannot be formed by the direct activation of the $\alpha-\mathrm{C}-\mathrm{H}$ bond due to the steric repulsion that results from the $\mathrm{CHOH}$ 
substitute. It is formed instead via the activation of the central $\mathrm{O}-\mathrm{H}$ bond which promotes the subsequent $\mathrm{C}-\mathrm{H}$ activation and equilibrates the dehydrogenation of 1,2-PDO to the corresponding $\mathrm{CH}_{3}\left(\mathrm{C}^{*}=\mathrm{O}^{*}\right)-\mathrm{CH}_{2} \mathrm{OH}$. The ketone can hydrogenate to form the hydroxyalkyl intermediate which is the active intermediate for breaking the $\mathrm{C}-\mathrm{O}$ bond. The path for the hydrogenolysis of the secondary $\mathrm{C}-\mathrm{OH}$ bond of 1,2 PDO summarized in Figure 2.10 is characteristically different from that for the hydrogenolysis of the terminal $\mathrm{C}-\mathrm{OH}$ as the terminal $\mathrm{C}-\mathrm{H}$ bond is not sterically hindered and can react to directly form the hydroxyalkyl intermediate which subsequently dissociates.

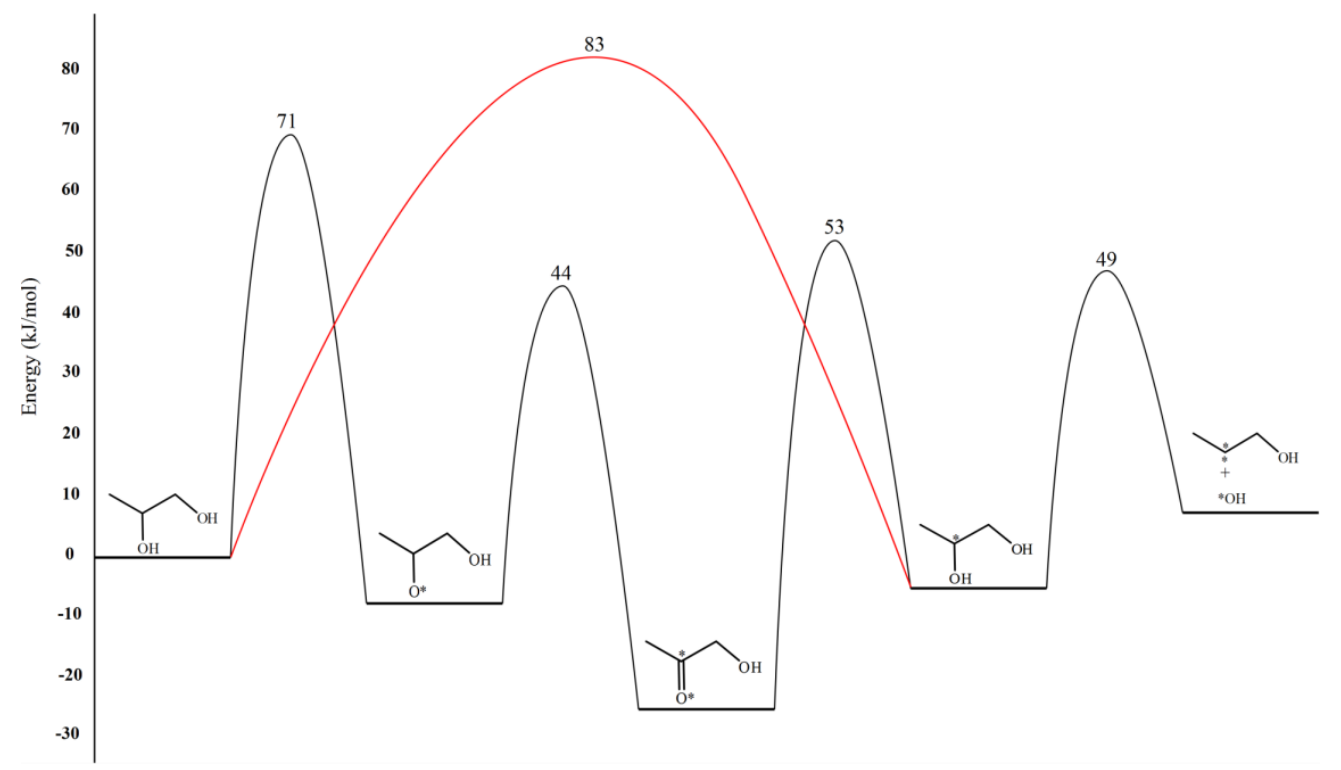

Figure 2.10 Energy diagrams for the plausible path of C-O breaking of 1,2-PDO at the center carbon.

\subsubsection{Kinetics Analysis of the hydrogenolysis of 1,2-PDO}

The energy diagrams for the plausible paths of the hydrogenolysis of 1,2-PDO at both the less substituted terminal carbon and the more substituted center carbon were shown in Figure 2.11. The adsorption energies, overall reaction energies and activation barriers for these 
elementary steps were used along with the estimates of the pre-exponential factors for each step to calculate the corresponding rate constants $(k)$ for each step. The elementary steps together with the corresponding rate constants were used along with the temperatures and partial pressures of the reactants as input to a microkinetic model to simulate the hydrogenolysis of 1,2-PDO to form 1-PO and 2-PO on the $\mathrm{Rh}(111)$ surface as a function of time.

The results from the simulations which are shown in Figure 2.12 indicate that at low $\mathrm{H}_{2}$ pressures the $\mathrm{Rh}(111)$ surface is covered by $\mathrm{OH}^{*}, \mathrm{CH}_{3} \mathrm{CH}(\mathrm{OH})-\mathrm{CH}^{*} \mathrm{OH}$ and $\mathrm{CH}_{3} \mathrm{C}^{*}=\mathrm{O}^{*}$ $\mathrm{CH}_{2} \mathrm{OH}$ intermediates with coverages of $0.56,0.41$ and 0.03 , respectively. This suggests that the hydrogenation of $\mathrm{OH}^{*}$ and the activation of the $\mathrm{C}-\mathrm{O}$ bond of $\mathrm{CH}_{3} \mathrm{CH}(\mathrm{OH})-\mathrm{CH}^{*} \mathrm{OH}$ are likely rate-limiting steps in the hydrogenolysis of 1,2-PDO at the terminal carbon and that the hydrogenation of $\mathrm{CH}_{3}\left(\mathrm{C}^{*}=\mathrm{O}^{*}\right)-\mathrm{CH}_{2} \mathrm{OH}$ limits the hydrogenolysis of 1,2-PDO at the center carbon. The higher selectivity to 2-PO over 1-PO indicates that the less substituted terminal carbon is more reactive than the more substituted center carbon of 1,2-PDO, which is consistent with the hydrogenolysis of cyclic ethers such as THFA over $\mathrm{Rh}$ where the $\mathrm{C}-\mathrm{O}$ bond is preferentially activated at the least-substituted carbon. ${ }^{[84]}$

Increasing the $\mathrm{H}_{2}$ pressure increases the $\mathrm{H}^{*}$ coverage on the $\mathrm{Rh}(111)$ surface. At high enough pressures, hydrogen will fully cover the entire surface, and block the hydrogenolysis reactions as the hydrogenolysis proceeds via dehydrogenation which requires empty sites to proceed. It is noted that these microkinetics analyses are based on simplisitic Langmuirian models and do not take into account coverage effects and multi-binding of the surface species. A more rigorous analysis would require kinetics Monte Carlo simulations. ${ }^{[105,106]}$ 


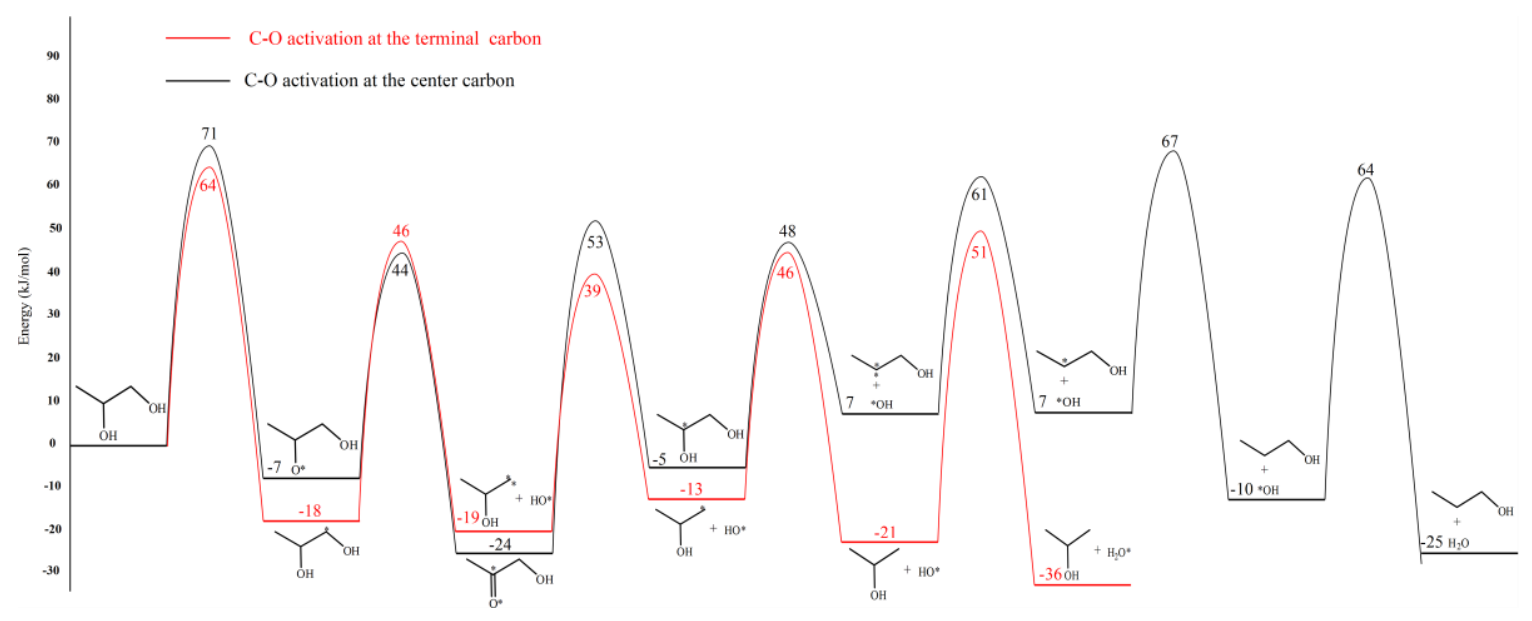

Figure 2.11 Reaction energy diagrams for the plausible paths of the hydrogenolysis of 1,2-PDO at the terminal carbon to form 2-PO (red) and at the center carbon to form 1-PO (black).
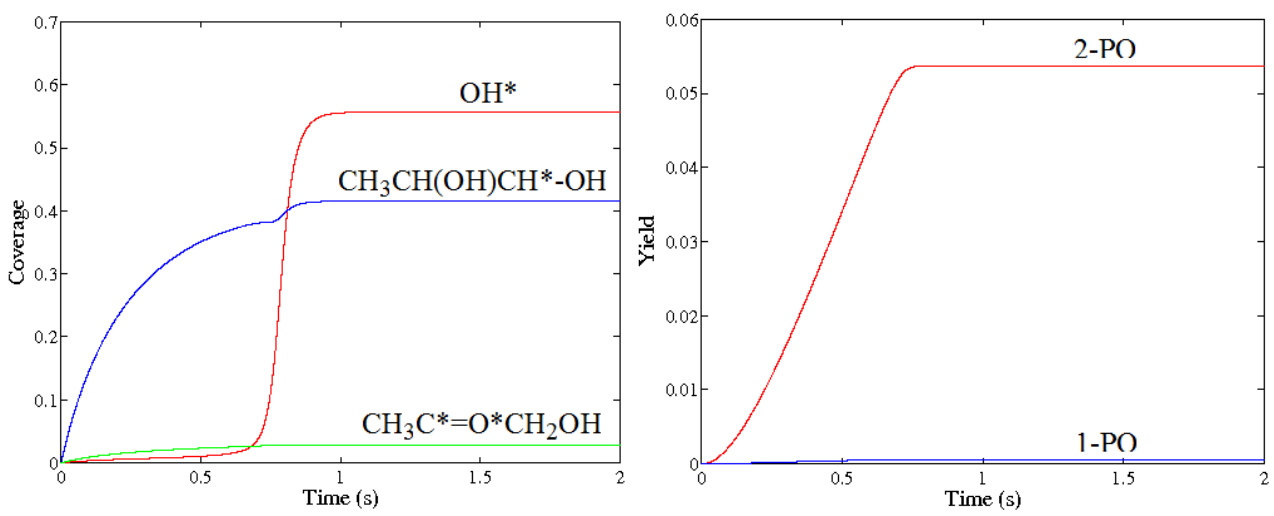

Figure 2.12 Coverage of several important intermediates on the $\mathrm{Rh}(111)$ surface (left) and the yield of 2-PO and 1-PO (right) during the kinetics simulation of the hydrogenolysis of 1,2-PDO.

\subsubsection{Effects of $\alpha-O H$ on the hydrogenolysis of 1,2-PDO}

The hydrogenolysis of mono-functional alcohol 1-PO and 2-PO were also examined and compared to the hydrogenolysis of the polyol 1,2-PDO, to gain insights as to the effects of the $\alpha$ $\mathrm{OH}$ substituents on the hydrogenolysis of polyols. We predominantly examined the representative $\mathrm{O}-\mathrm{H}$ activation, $\mathrm{C}-\mathrm{H}$ activation and $\mathrm{C}-\mathrm{O}$ activation steps discussed above for the hydrogenolysis of both 1-PO and 2-PO for comparison. 
1-PO adsorbs via its $\mathrm{OH}$ group and reacts via a metal insertion into the $\mathrm{O}-\mathrm{H}$ bond as shown in Figure 2.13. The transition state structure is very similar to that for the $\mathrm{O}-\mathrm{H}$ activation of 1,2PDO at the terminal carbon. The energy barrier is calculated to be $81 \mathrm{~kJ} / \mathrm{mol}$, which is about 14 $\mathrm{kJ} / \mathrm{mol}$ higher than the activation of the terminal $\mathrm{O}-\mathrm{H}$ bond of 1,2-PDO. The lower barrier for 1,2-PDO is likely the result of the intramolecular hydrogen bond that forms with the terminal O$\mathrm{H}$ group to help stabilize the transition state. By rotating the $\mathrm{OH}$ group in the 1,2-PDO we can artificially eliminate the hydrogen bond. While the terminal $\mathrm{O}-\mathrm{H}$ activation barrier increases to $70 \mathrm{~kJ} / \mathrm{mol}$, it is still $\sim 11 \mathrm{~kJ} / \mathrm{mol}$ lower than that for the $\mathrm{O}-\mathrm{H}$ bond activation of 1 -PO. From Figure 2.13, we can see that the activation of the terminal $\mathrm{O}-\mathrm{H}$ of 1,2-PDO, results in a significant decrease in the $\mathrm{Rh}-\mathrm{OH}$ distance at the central $\mathrm{C}$ from $2.53 \AA$ in the reactant to about $2.36 \AA$ in the transition state. This suggests a strong stabilization of the transition state via the interaction of the central $\mathrm{OH}$ bond and the $\mathrm{Rh}$ surface.

A detailed charge analysis along the reaction coordinate for the activation of the terminal $\mathrm{O}$ $\mathrm{H}$ bond of 1,2-PDO indicates that the charge of the metal surfaces changes from -0.23 in the reactant to +0.02 in the transition state as a result of electron transfer from the metal into the antibonding $\mathrm{O}-\mathrm{H}$ state to activate the terminal $\mathrm{O}-\mathrm{H}$ bond of 1,2-PDO in the transition state. The charge of the $\mathrm{Rh}$ atom which inserts into the terminal $\mathrm{O}-\mathrm{H}$ bond changes from +0.04 in the reactant to +0.13 in the transition state as result of the back-donation. The charge on the vicinal $\mathrm{Rh}$ atom which binds to the oxygen at the center carbon, changes from +0.03 to +0.08 . This increase in positive charge at the vicinal Rh atom allows for increased electron donation from the central $\mathrm{O}$ and thus stronger interaction between the secondary $\mathrm{C}-\mathrm{OH}$ group and the vicinal $\mathrm{Rh}$ site. This increased interaction in the transition state acts to lower the barrier for the activation of the terminal O-H bond of 1,2-PDO. 

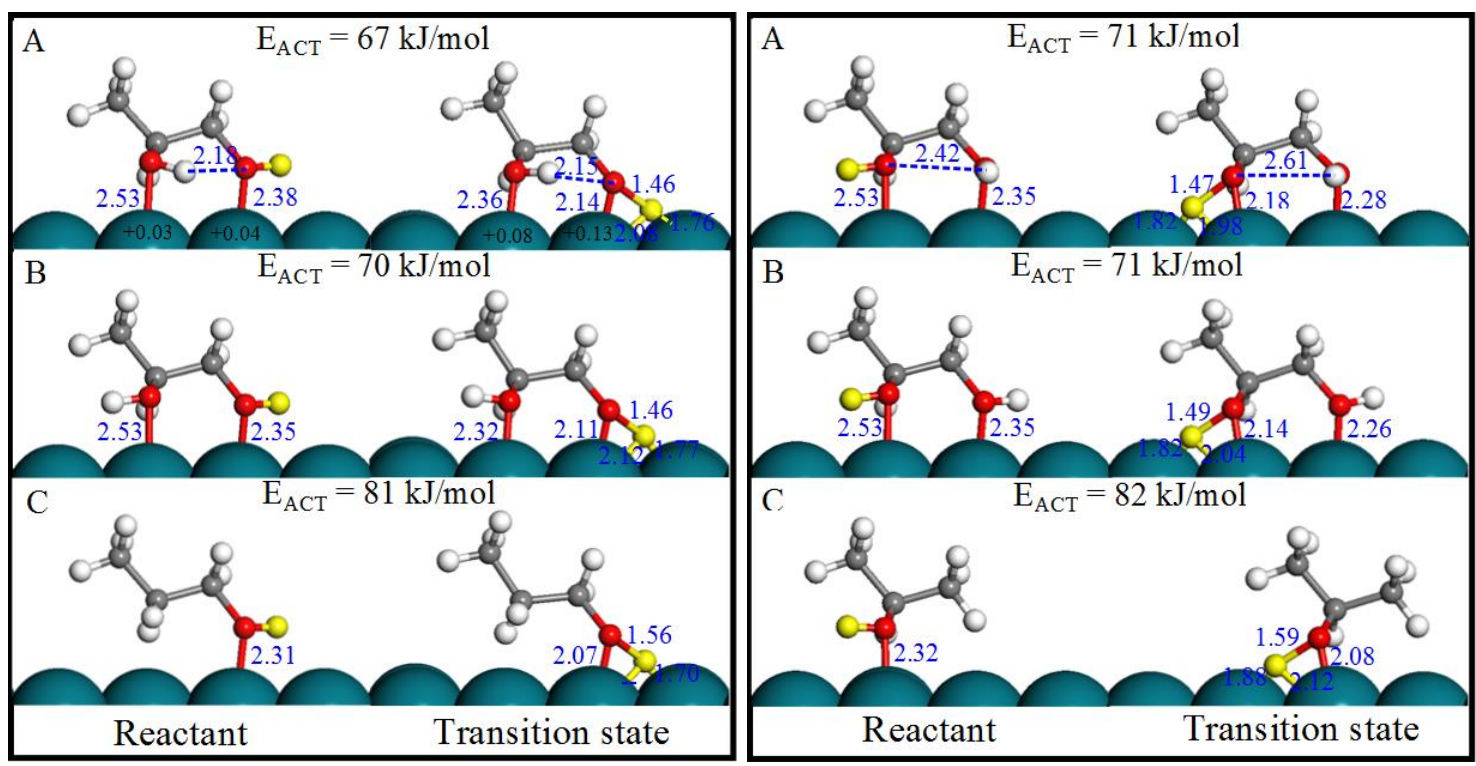

Figure 2.13 DFT calculated reactant and transition state structures for the O-H activation of 1,2PDO at the terminal carbon with intramolecular hydrogen bond (left-A), without intramolecular hydrogen bond (left-B) and $\mathrm{O}-\mathrm{H}$ activation of 1-PO (left-C); O-H activation of 1,2-PDO at the center carbon with intramolecular hydrogen bond (right-A), without intramolecular hydrogen bond (right-B) and $\mathrm{O}-\mathrm{H}$ activation of 2-PO (right-C).

The barrier to activate the $\mathrm{O}-\mathrm{H}$ bond of $2-\mathrm{PO}$ was calculated to be $82 \mathrm{~kJ} / \mathrm{mol}$, which is 11 $\mathrm{kJ} / \mathrm{mol}$ higher than that to activate the central $\mathrm{O}-\mathrm{H}$ of 1,2-PDO. A close analysis of the structures shown in Figure 2.13 reveals a decrease in the $\mathrm{Rh}-\mathrm{OH}$ bond distance between the vicinal $\mathrm{Rh}$ and the terminal $\mathrm{OH}$ group as the central $\mathrm{O}-\mathrm{H}$ bond is activated. This results in a stronger $\mathrm{Rh}-\mathrm{OH}$ bond between the vicinal $\mathrm{Rh}$ and the terminal $\mathrm{OH}$ group in the transition state which stabilizes the transition state and lowers the barrier.

The transition state structure for the activation of the $\alpha-\mathrm{C}-\mathrm{H}$ bond of $1-\mathrm{PO}(74 \mathrm{~kJ} / \mathrm{mol})$ is similar to that for the activation of the $\alpha-\mathrm{C}-\mathrm{H}$ bond of $1,2-\mathrm{PDO}(64 \mathrm{~kJ} / \mathrm{mol})$ at the terminal carbon as shown in Figure 2.14 but the barrier is $\sim 12 \mathrm{~kJ} / \mathrm{mol}$ higher. If we reorient the terminal $\mathrm{OH}$ group to eliminate the intramolecular hydrogen bond between the two - OHs as shown in Figure 2.14, the barrier to activate the $\alpha-\mathrm{C}-\mathrm{H}$ bond of 1,2-PDO increases to $78 \mathrm{~kJ} / \mathrm{mol}$, which is 
very close to that for the activation of the $\alpha-\mathrm{C}-\mathrm{H}$ bond of 1-PO. This indicates that the lower barrier to activate the $\alpha-\mathrm{C}-\mathrm{H}$ bond of 1,2-PDO at the terminal carbon is due to the stabilization that results from the formation of an intramolecular hydrogen bond that stabilizes the transition state.

The same trend was found in comparing the activation of the central $\mathrm{C}-\mathrm{H}$ bond of 2-PO and 1,2-PDO. The barrier to activate the central $\mathrm{C}-\mathrm{H}$ bond of $1,2-\mathrm{PDO}(83 \mathrm{~kJ} / \mathrm{mol})$ is $8 \mathrm{~kJ} / \mathrm{mol}$ lower than that to activate the central $\mathrm{C}-\mathrm{H}$ bond of $2-\mathrm{PO}(91 \mathrm{~kJ} / \mathrm{mol})$. The decrease is due to the stabilization of the transition state for the 1,2-PDO via the formation of an intramolecular hydrogen bond.

The barrier to activate the second $\alpha-\mathrm{C}-\mathrm{H}$ bond of $1-\mathrm{PO}$ was calculated to be $49 \mathrm{~kJ} / \mathrm{mol}$, which is much lower than barrier to activate the second $\mathrm{C}-\mathrm{H}$ bond activation of 1,2-PDO at the terminal carbon $(80 \mathrm{~kJ} / \mathrm{mol})$. As discussed earlier, the higher barrier associated with activating the second $\mathrm{C}-\mathrm{H}$ bond of $1,2-\mathrm{PDO}$ at the terminal carbon is due to the weak binding of $-\mathrm{OH}$ at the center carbon to the metal surface as well as the weak intramolecular hydrogen bonding in the transition state than in the reactant. This is due to the fact that we must break the strong interaction of the $-\mathrm{OH}$ at central carbon and the $\mathrm{Rh}$ surface as we move from the reactant to the transition state. The lower barrier for the activation of the second $\alpha-\mathrm{C}-\mathrm{H}$ bond in 1-PO helps to promote the subsequent activation of the $\mathrm{C}-\mathrm{O}$ breaking which is similar to the results found for the C-O activation of cyclic ether THFA that we reported previously. ${ }^{[84]}$ 


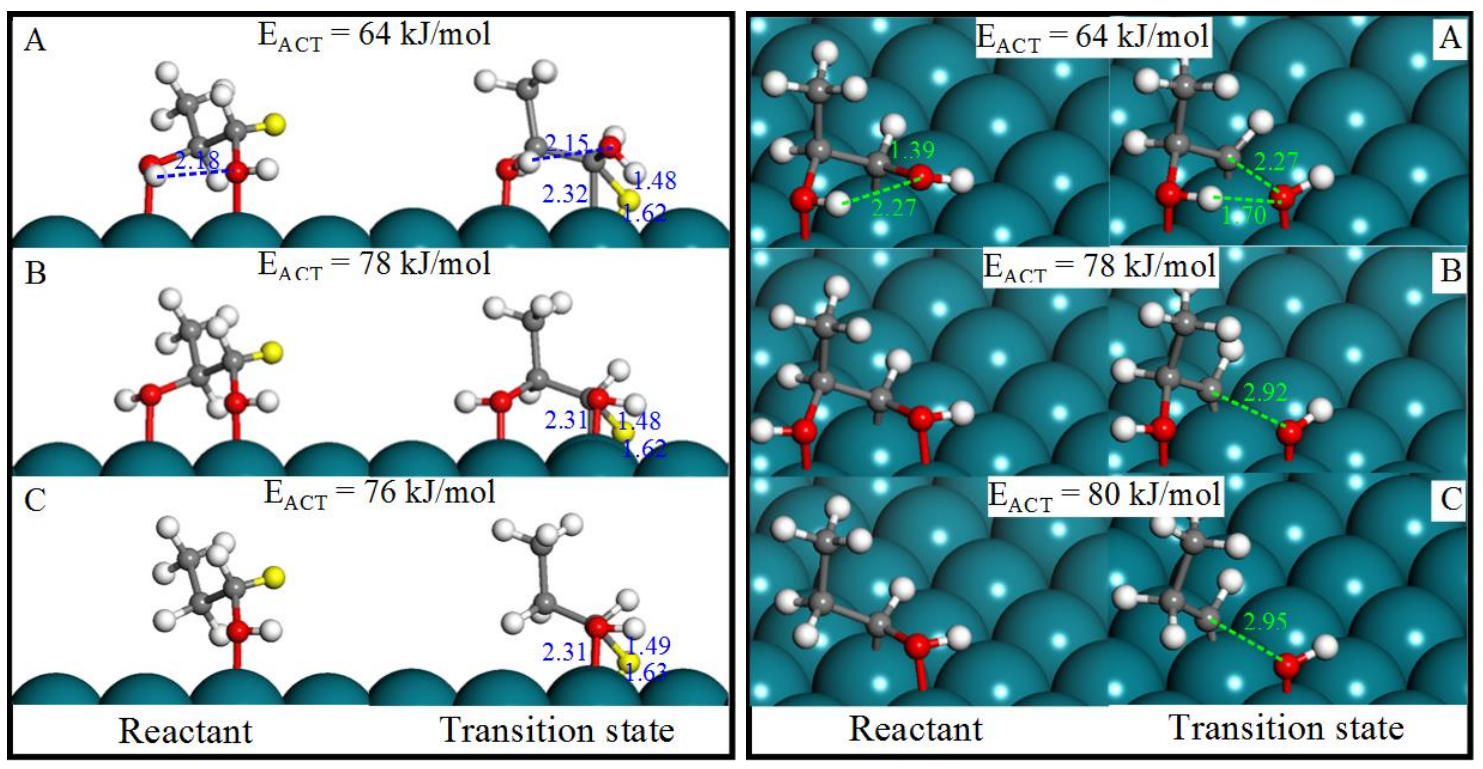

Figure 2.14 DFT-calculated reactant and transition state structures for the C-H activation of 1,2PDO at the terminal carbon with intramolecular hydrogen bond (left-A), without intramolecular hydrogen bond (left-B) and the $\mathrm{C}-\mathrm{H}$ activation of 1-PO at the terminal carbon (left-C); C-O activation of the intermediate $\mathrm{CH}_{3} \mathrm{CH}(\mathrm{OH}) \mathrm{CH}^{*} \mathrm{OH}$ at the terminal carbon with intramolecular hydrogen bond (right-A), without intramolecular hydrogen bond (right-B) and $\mathrm{C}-\mathrm{O}$ activation of the intermediate $\mathrm{CH}_{3} \mathrm{CH}_{2} \mathrm{CH}^{*} \mathrm{OH}$ (right-C).

The activation of the $\mathrm{C}-\mathrm{OH}$ bond of the hydroxylalkyl intermediate $\mathrm{CH}_{3} \mathrm{CH}_{2} \mathrm{CH}^{*}-\mathrm{OH}$ formed via activation of the terminal $\mathrm{C}-\mathrm{H}$ bond of 1-PO was examined as shown in Figure 2.14. The activation barrier of $80 \mathrm{~kJ} / \mathrm{mol}$ is $16 \mathrm{~kJ} / \mathrm{mol}$ higher than the barrier to activate the terminal C$\mathrm{OH}$ bond of the $\mathrm{CH}_{3} \mathrm{CH}(\mathrm{OH}) \mathrm{CH}^{*}-\mathrm{OH}$ hydroxyalkyl intermediate $(64 \mathrm{~kJ} / \mathrm{mol})$ formed via $\mathrm{C}-\mathrm{H}$ activation of 1,2-PDO. The lower barrier involved in the activating the $\mathrm{C}-\mathrm{OH}$ from the 1,2-PDO intermediate is due to the stabilization that results from the intermolecular hydrogen bonding between two -OHs of 1,2-PDO that stabilizes the transition state as shown in Figure 2.14. By rotating the secondary $\mathrm{O}-\mathrm{H}$ group, we remove the intramolecular hydrogen bond, and the activation energy to activate the terminal $\mathrm{C}-\mathrm{OH}$ bond of the $\mathrm{CH}_{3} \mathrm{CH}(\mathrm{OH}) \mathrm{CH}^{*}$-OH hydroxyalkyl intermediate increases to $78 \mathrm{~kJ} / \mathrm{mol}$, which is very close to that for the activation of the $\mathrm{C}-\mathrm{OH}$ bond of the $\mathrm{CH}_{3} \mathrm{CH}_{2} \mathrm{CH}^{*}-\mathrm{OH}$ hydroxyalkyl intermediate formed from 1-PO. 
By comparing the mono-functional 1-PO and 2-PO alcohols with that for the 1,2-PDO polyol, we can see that the presence of neighboring $\alpha-\mathrm{OH}$ groups can facilitate the $\mathrm{O}-\mathrm{H}, \mathrm{C}-\mathrm{H}$ as well as $\mathrm{C}-\mathrm{OH}$ bond activation from the polyols as a result of the intermolecular hydrogen bonding and via the formation of stronger metal-OH bond at the $\alpha-\mathrm{OH}$ position thus aiding in the hydrogenolysis of the polyols over the monofunctional alcohols.

\subsection{Conclusions}

We systematically examined the possible pathways that control the hydrogenolysis of polyol 1,2-PDO over the $\mathrm{Rh}(111)$ surface, and determined the most plausible paths to form the 1-PO and 2-PO products. The results show that the hydrogenolysis of 1,2-PDO predominantly proceeds at the terminal carbon to form 2-PO. The aldehyde intermediate $\mathrm{CH}_{3} \mathrm{CH}(\mathrm{OH}) \mathrm{CH}=\mathrm{O}$ is readily formed via the activation of the of the $\mathrm{C}-\mathrm{H}$ and $\mathrm{O}-\mathrm{H}$ bonds of the terminal carbon. The aldehyde, however, does not appear to favorably undergo C-O bond breaking. The C-O bond breaking of 1,2-PDO at the terminal carbon proceeds instead via the initial activation of the terminal $\mathrm{C}-\mathrm{H}$ bond of 1,2-PDO to form the reactive $\mathrm{CH}_{3} \mathrm{CH}(\mathrm{OH}) \mathrm{CH}^{*} \mathrm{OH}$ hydroxyalkyl intermediate which subsequently undergoes $\mathrm{C}-\mathrm{OH}$ activation. The activation of the center $\mathrm{C}-\mathrm{OH}$ bond of 1,2-PDO to form 1-PO also proceeds through the formation of the reactive $\mathrm{CH}_{3} \mathrm{C}^{*}(\mathrm{OH})$ $\mathrm{CH}_{2} \mathrm{OH}$ hydroxyalkyl intermediate. The reaction cannot occur via the direct activation of the secondary $\mathrm{C}-\mathrm{H}$ bond due to the steric inhibition of the central $\mathrm{C}-\mathrm{H}$ bond. The reaction instead proceeds via the dehydrogenation of the central $\mathrm{C}-\mathrm{OH}$ bond of 1,2-PDO followed by the subsequent hydrogen addition to the ketone intermediate to form the secondary hydroxyalkyl intermediate $\left(\mathrm{CH}_{3} \mathrm{C}^{*}(\mathrm{OH})-\mathrm{CH}_{2} \mathrm{OH}\right)$. Microkinetic analyses for the hydrogenolysis of 1,2-PDO on the $\mathrm{Rh}(111)$ surface at low hydrogen pressures indicate that $\mathrm{OH}^{*}$ as well as 
$\mathrm{CH}_{3} \mathrm{CH}(\mathrm{OH}) \mathrm{CH} * \mathrm{OH}$ cover the $\mathrm{Rh}$ surface and that the hydrogenation of $\mathrm{OH}^{*}$ and the $\mathrm{C}-\mathrm{O}$ activation of $\mathrm{CH}_{3} \mathrm{CH}(\mathrm{OH}) \mathrm{CH} * \mathrm{OH}$ are likely to be the rate-limiting steps. The higher selectivity to form 2-PO over 1-PO results from the fact that the less substituted terminal carbon is more reactive than the central $\mathrm{C}$ in the hydrogenolysis of 1,2-PDO on $\mathrm{Rh}(111)$ surface.

A comparison of the hydrogenolysis of 1,2-PDO with the mono-functional 1-PO and 2-PO indicates that the $\alpha-\mathrm{OH}$ in 1,2-PDO facilitates important $\mathrm{O}-\mathrm{H}, \mathrm{C}-\mathrm{H}$ as well as $\mathrm{C}-\mathrm{OH}$ activation steps in the hydrogenolysis process. 


\section{Chapter 3-Selective Hydrogenolysis of Tetrahydrofurfural Alcohol over Rh-Re Catalyst}

\subsection{Introduction}

The hydrogenolysis of cyclic ethers such as THFA and polyols such as 1,2-propanediol over $\mathrm{Rh}$ and other reducible transition metal catalysts tend to proceed via the activation of $\mathrm{C}-\mathrm{O}$ bonds at the less substituted carbon centers as was just described in Chapter 2 and reported previously in my Master's thesis ${ }^{[84]}$. This trend in selectivity, as well as the activity, is significantly altered by the addition of oxophilic metals such as Re or Mo to the Rh and other reducible transition metal catalysts including $\mathrm{Pt}, \mathrm{Pd}$ and $\mathrm{Ir}^{\left[{ }^{[72]}\right.}$ These alloyed catalysts show increases in the activity and marked changes in the selectivity as $\mathrm{C}-\mathrm{O}$ activation tends to predominantly proceed at the more substituted carbon centers during the hydrogenolysis of THFA, 1,2-propenediol and other cyclic ethers and polyols. ${ }^{[72]}$ Hydrogenolysis over these promoted alloys does not appear to proceed via the metal catalyzed mechanism discussed in Chapter 2 as it cannot describe the observed trends in selectivity. The results suggest that a different mechanism is responsible for the hydrogenolysis of THFA and other cyclic ethers and polyols over the supported Rh-Re catalysts.

In order to explore the role of the Re promoter on the changes that occur in the hydrogenolysis activity and selectivity of cyclic ethers and polyols, we first examine the state of Re in the active catalyst. Detailed characterizations of the Rh-Re catalysts indicate that while $\mathrm{Rh}$ is fully reduced, Re is well dispersed resulting in an Rh-Re alloy but is not fully reduced. ${ }^{[62,}$

${ }^{73]}$ Temperature-programmed $\mathrm{NH}_{3}$ desorption experiments indicate that $\mathrm{NH}_{3}$ binds rather strongly on $\mathrm{Rh}-\mathrm{Re} / \mathrm{C}$ catalysts with adsorption energies of about $-100 \mathrm{~kJ} / \mathrm{mol} .{ }^{[72,73]}$ This suggests that the addition of Re can lead to the formation of acidic sites on the Rh-Re catalysts, which is further 
confirmed by the fact that the addition of a base such as $\mathrm{NaOH}$ quenches the catalytic activity of the Rh-Re catalyst in the hydrogenolysis of THFA. ${ }^{[72]}$ In further support for the presence of acid sites, Dumesic et al. ${ }^{[73]}$ recently showed that the Rh-Re catalysts was quite active in the Brønsted acid catalyzed dehydration of fructose to 5-hydroxymethylfurfural (HMF) and found similar HMF selectivities as compared to the well-known solid acid catalyst ZSM-5. This indicates that the strength of the Brønsted acid sites over the Rh-Re catalyst and ZSM-5 are similar as the HMF selectivity has been suggested to correlate with the strength of Brønsted acid sites over solid acid catalysts. ${ }^{[112,113]}$

While other studies provide some insights into the possible structure and composition of these acid sites, recent work by Dumesic et al. ${ }^{[73]}$ found that water is essential for the activity of the Rh-Re catalyst in the hydrogenolysis of hydroxyl-methyl-tetrahydropyran (HMTHP) and that the reaction rate of the hydrogenolysis of HMTHP on Rh-Re catalyst is linearly correlated to the water concentration of the water-THF mixture solvent. They indicate that there are metallic Re atoms on the surface of the Rh-Re alloy catalyst as a result of XANES and EXAFS information and that the presence of rhenium oxide does not appear to be necessary to impart the acidic functionality to the Rh-Re catalyst. ${ }^{[73]}$ Based on these experimental observations, they suggested that the acid sites on the $\mathrm{Rh}-\mathrm{Re}$ catalyst may be generated from the activation of water molecules over the Re atoms on the surface of metallic Rh-Re particles. ${ }^{[73]}$ Tomishige et al. ${ }^{[69]}$ recently examined the hydrogenolysis of THFA on the Ir-Re catalyst in n-heptane rather than water, and found the reaction rate in the alkane solvent is about 10 times lower than that found in water and the selectivity to 1,5 -pentanediol decreases from about $97 \%$ in water to about $50 \%$ in the alkane solvent. This would also help to confirm that water is necessary to generate the acid sites on the Rh-Re and Ir-Re catalyst. 
In light of these observations, it appears that the higher selectivity to activate the $\mathrm{C}-\mathrm{O}$ bonds at the more substituted C-centers may be the result of an acid catalyzed mechanism on the Rh-Re catalyst. At present, little is known about the molecular structure of the responsible acid site or the mechanism by which it catalyzes hydrogenolysis of cyclic ethers and polyols. The current focus of the work is then to explore these two issues using theoretical methods to model the acid site and the reaction mechanism at the atomic level.

In our initial theoretical calculations, we suggested that the acid sites of the Rh-Re catalyst may be the result of hydroxyl groups that bind strongly to the Re sites on the Rh-Re alloy surface, which can be generated via the activation of water on the surface of Rh-Re alloy. ${ }^{[72]}$ This is consistent with XANES results which indicates that the Re atoms maintain a partial positive charge ${ }^{[62]}$ The DFT calculations also showed the deprotonation energies (DPE) of the Re-OH site on the Rh-Re alloy surface is comparable to that of the acidic zeolites, which is consistent with the experimental observations as the deprotonation energy is a measure of the acidity of Brønsted acids. ${ }^{[73]}$ Herein, we examine the mechanisms for the hydrogenolysis of THFA over the model $\mathrm{Rh}-\mathrm{Re}$ surfaces where $\mathrm{Re}-\mathrm{OH}$ ensembles were used as models of the acid site. The mechanistic insights for the hydrogenolysis of THFA were subsequently extended to a range of other cyclic ethers and polyols.

\subsection{Computational methods}

All of the calculations reported herein were carried out using periodic plane-wave gradientcorrected density functional theory methods implemented in the Vienna ab initio Simulation Package (VASP). ${ }^{[89-92]}$ The PW91 ${ }^{[93]}$ form of generalized gradient approximation (GGA) exchange correlation functional was used to calculate the non-local gradient-corrections to 
exchange and correlation energies. Wave functions were constructed by using projector augmented wave potentials (PAW) ${ }^{[94,95]}$ within a cutoff energy of $396.0 \mathrm{eV}$.

Herein we used the model $\mathrm{Rh}(111)$ surface along with well-dispersed $\mathrm{Re}-\mathrm{OH}$ groups as shown in Figure 3.1 (left) to provide a simple model of the Brønsted acid promoted metal surfaces that occur on the supported Rh-Re catalysts. The Rh(111) surface was modeled using a 4x4 unit cell with four metal layers and a $15 \AA$ vacuum region above the metal layers, where the top two layers were allowed to relax and the bottom two held fixed to their lattice constant for $\mathrm{Rh}$. The solution phase above the Rh surface was modeled by allowing 40 water molecules to fill the vacuum region above the surface in order to establish a density of water that is close to 1 $\mathrm{g} / \mathrm{cm}^{3}$. Three water molecules around the acid site were removed to provide the space necessary to activate the $\mathrm{C}-\mathrm{O}$ bond of THFA. Ab initio molecular dynamics (NVT ensemble) simulations with all the metal atoms fixed were carried out at $400 \mathrm{~K}$ for $2.0 \mathrm{ps}$ with the time step of $1.0 \mathrm{fs}$ to

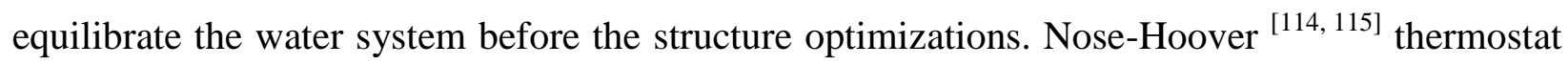
was used to control the temperature during the run. A 6x6x1 Monkhorst-Pack ${ }^{[96]} \mathrm{k}$-point mesh to sample the first Brillouin zone and the electronic energies were converged to a tolerance of $1 \times 10^{-6} \mathrm{eV}$. All of the structural optimizations were carried out until the forces on all of the atoms were optimized to within $0.05 \mathrm{eV} \AA^{-1}$. For the ab initio molecular dynamics simulations, a $3 \times 3 \times 1$ Monkhorst-Pack ${ }^{[96]} \mathrm{k}$-point mesh was used, and the electronic energies were converged to within $1 \times 10^{-4} \mathrm{eV}$.

The $\mathrm{Rh}_{201}$ cubo-octahedral particle was further used to provide a more rigorous and reliable model of the acid sites on the Rh-Re catalysts where we explore the $\mathrm{Re}-\mathrm{OH}$ site at various positions including the 111 and 100 terrace, edge and corner sites on the $\mathrm{Rh}_{201}$ particle shown in Figure 3.1. The calculations on the metal particles were all carried out without spin-polarization 
in a $30.44 \AA$ cubic unit cell with a minimum of $12 \AA$ of vacuum separating particles between periodic cells. The electronic and geometric structures were converged to within the same criterion as those reported above for the $\mathrm{Re}-\mathrm{OH}$ promoted $\mathrm{Rh}(111)$ surfaces. These calculations were performed using the $\gamma$-point version of VASP and the $\Gamma$ k-point mesh was used to sample the first Brillouin zone. The dipole moments of the cell were calculated and used to correct the energy. For charged calculations, quadrupole corrections were also applied. The energies of the adsorbates in vacuum were calculated spin-polarized in an 18x18x18 ̊̊ cubic unit cell.

The transition states were isolated using the nudged elastic band (NEB). ${ }^{[98]}$ and dimer simulations. ${ }^{[97]}$ The minimum energy path was estimated using the NEB calculations with 16 intermediates converged until the force normal to the reaction path was less than $0.25 \mathrm{eV} \AA^{-1}$. The results from the NEB simulations were used to initiate the dimer calculation, which uses a pair of closely-spaced structures to search for a saddle point on the potential energy surface. The dimer calculations were converged until the normal force was less than $0.05 \mathrm{eV} \AA^{-1}$ with the same k-point mesh and the same convergence criterion for electronic energies as the optimization calculations.

Detailed charge analyses were carried out by using the QUAMBO method, ${ }^{[99-102]}$ which transforms the wave-functions of the VASP calculation into spatially localized non-orthogonal quasi-atomic orbitals. This transformation provides information about the atomic orbitals of the system, and permits a Löwdin charge and bond order analysis. ${ }^{[103,104]}$

The binding energies of the adsorbates at the acid sites or metal sites were calculated as:

$$
\Delta E_{a d s}=E_{M+i}-E_{M}-E_{i}
$$

where $E_{M+\mathrm{i}}$ is the total energy of the acid or metal with the adsorbate bound to it, $E_{M}$ is the total energy of the bare acid or metal and $E_{i}$ is the total energy of the adsorbate $i$ in gas phase. 
The activation barriers and reaction energies were calculated as:

$$
\begin{gathered}
\Delta E_{A C T}=E_{T S}-E_{R} \\
\Delta E_{r x n}=E_{P}-E_{R}
\end{gathered}
$$

respectively, where $E_{T S}$ is the total energy of the transition state, $E_{R}$ is the total energy of the reactant state and $E_{P}$ is the total energy of the product state.

The gas phase carbenium ion formation energies for ring structures such as THFA and MTHF were calculated as:

$$
\Delta E_{\mathrm{rxn}(\mathrm{R}+)}=E_{R O R H^{+}}-E_{\mathrm{ROR}}-E_{H^{+}}
$$

where $E_{R O R H^{+}}$is the total energy of the ring opened carbenium ion, $E_{R O R}$ is the total energy of the initial ring structure before the ring opening, $E_{H^{+}}$is the total energy of the proton.

Similarly, the gas phase carbenium ion formation energies for polyols and mono-functional alcohols such as 1,2-propanediol and 2-propanol were calculated as:

$$
\Delta E_{\mathrm{rxn}(\mathrm{R}+)}=E_{\mathrm{RH}}+E_{\mathrm{H} 2 \mathrm{O}}-E_{\mathrm{ROH}}-E_{\mathrm{H}^{+}}
$$

where $E_{R H^{+}}$is the total energy for the dehydrated carbenium ion, $E_{R O H}$ is the total energy of polyols or alcohols before dehydration, $E_{\mathrm{H} 2 \mathrm{O}}$ is the total energy of water and $E_{\mathrm{H}^{+}}$is the total energy of proton.
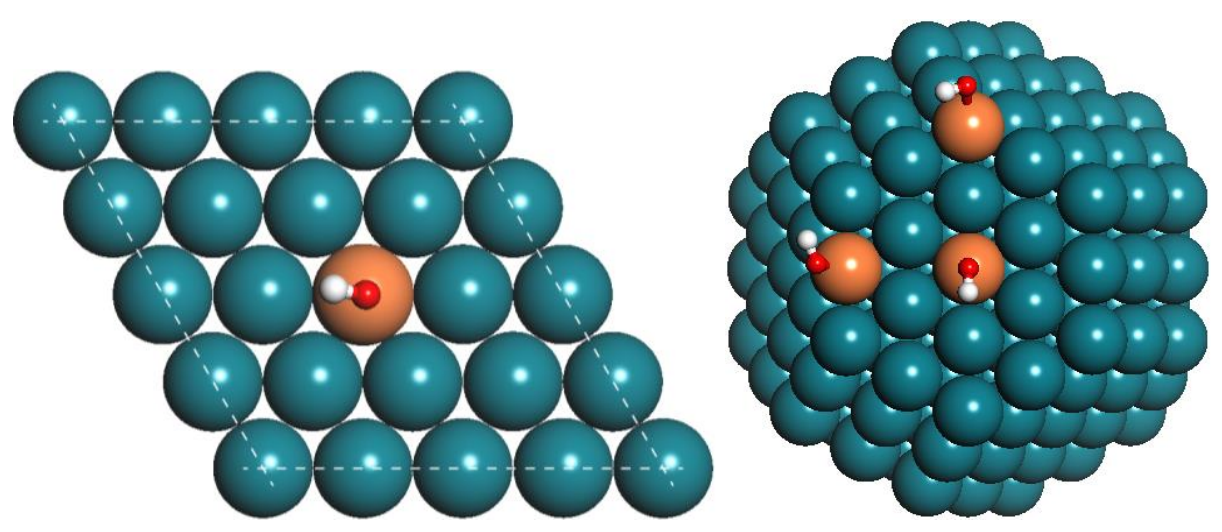

Figure 3.1 The $4 \times 4 \mathrm{Rh}(111)$ surface with well dispersed $\mathrm{Re}-\mathrm{OH}$ site (left) and the 201-atom cubo-octahedral Rh particle with Re-OH located at different sites (right) used to model the acid sites on Rh-Re catalyst. 


\section{Results and Discussions}

\subsection{Water activation on the Rh-Re alloy surface to generate acid site}

Water binds at the Re atom on the Rh-Re alloy surface through its oxygen with the binding energy of $-42 \mathrm{~kJ} / \mathrm{mol}$ as shown in Figure 3.2. The adsorbed water is activated by metal atom insertion into the $\mathrm{O}-\mathrm{H}$ bond resulting in the formation of a hydroxyl that binds at the $\mathrm{Re}$ atom with rather low activation barrier of $54 \mathrm{~kJ} / \mathrm{mol}$ as shown in Figure 3.2. For comparison, the barrier to activate water on the metal $\mathrm{Rh}(111)$ surface alone is considerably higher at $92 \mathrm{k} / \mathrm{mol}$, thus indicating that the oxophilic Re site in the Rh-Re alloy significantly promotes the activation of water to generate acid sites. In the presence of solution, the adsorbed water can dissociate heterolytically to form an $\mathrm{OH}(-)$ intermediate bound to a $\mathrm{Re}$ site on the $\mathrm{Rh}-\mathrm{Re}$ alloy, and proton in solution with a much lower activation barrier $(13 \mathrm{~kJ} / \mathrm{mol})$ as will be discussed in more detail in the next chapter. The strong adsorption of water and the very low activation barrier for its heterolytic dissociation to form $\mathrm{Re}-\mathrm{OH}$ indicates that the acid sites are readily formed at the exposed Re centers on the Rh-Re alloy surface. The easy oxidation of the metallic Re on the bimetallic alloy surface such as Pt-Re by water has also been observed in the water-gas-shift reaction experiments. ${ }^{[116,117]}$ 


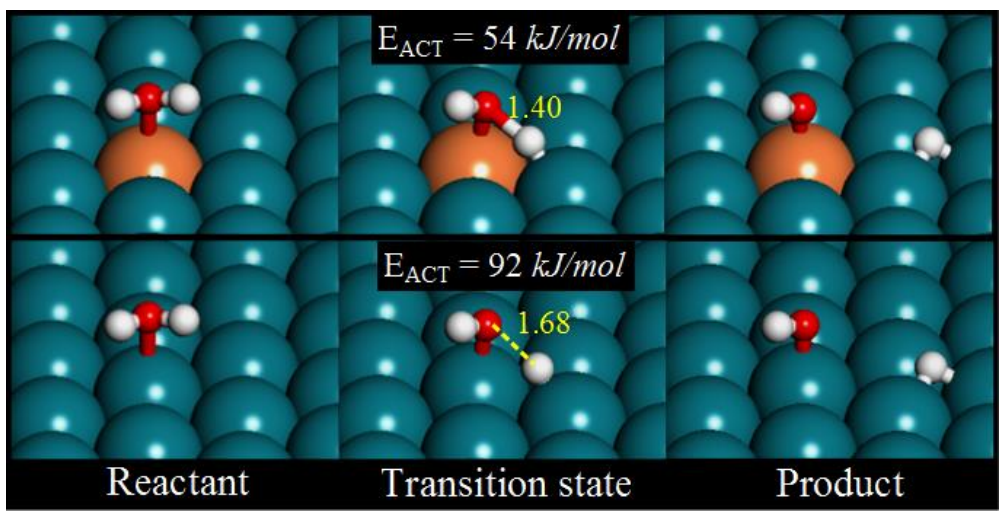

Figure 3.2 DFT calculated reactant, transition state and product structures for the dissociation of water on the $\mathrm{Rh}-\mathrm{Re}$ and pure $\mathrm{Rh}(111)$ surfaces.

\subsection{Ring opening of THFA at the Re-OH acid sites}

A detailed analysis of the hydrogenolysis of various cyclic ethers and polyols carried out over the supported Rh-Re catalysts indicates hydroxyl groups that sit $\alpha$ to the $\mathrm{C}-\mathrm{O}$ bond that is activated can significantly influence the selectivity and activity for hydrogenolysis. In comparing methyl-tetrahydrofuran (MTHF) and tetrahydrofurfural alcohol (THFA), there is a 30 fold increase in the hydrogenolysis activity that results from changing the $\alpha-\mathrm{H}$ on MTHF to an $\mathrm{OH}$ group to form THFA as well as a marked difference in product selectivity over the Rh-Re catalyst. ${ }^{[72]}$ To better understand the mechanism for the ring opening of THFA and the influence of the $\alpha-\mathrm{C}-\mathrm{OH}$ groups, we carry out Born-Haber cycle analysis which allows us to relate the changes in the activation barrier to the changes in the catalyst or changes in the molecules reacted. More specifically, the energy barrier $\left(\Delta E_{\mathrm{ACT}}\right)$ for a reaction can be written in terms of the deprotonation energy $(D P E)$ of the solid acid, which provides a measure of acidity, the gas-phase carbenium ion formation energy $\left(\Delta E_{\mathrm{rxn}(\mathrm{R}+)}\right)$, and the interaction energy $\left(\Delta E_{\text {int }}\right)$ between the gasphase carbenium ion and the negative charge of the conjugate base that forms in the transition state: ${ }^{[118,119]}$ 


$$
\Delta E_{\mathrm{ACT}}=D P E+\Delta E_{\mathrm{rxn}(\mathrm{R}+)}+\Delta E_{\mathrm{int}}
$$

Different reactions carried out over the same catalyst can then be described simply by the changes in the gas-phase carbenium ion formation energies $\left(\Delta E_{\mathrm{rxn}(\mathrm{R}+)}\right)$ and changes in the interaction energies $\left(\Delta E_{\text {int }}\right)$. The changes in the interaction energies that result from different reactants are often linearly related to the changes in the gas-phase carbenium ion formation energies at the same solid acid catalyst. ${ }^{[120]}$ The changes of activation barriers for different reactants $\Delta\left(\Delta E_{a c t}\right)$ can then be linearly correlated to the changes in their gas phase carbenium ion formation energies $\Delta\left(\Delta E_{\mathrm{rxn}(\mathrm{R}+)}\right)$. As shown in Figure 3.3, the high selectivity for the ring opening of THFA to the $\alpha, \omega$-diol 1,5-pentanediol observed by the experiments can be attributed to the greater stability $(30 \mathrm{~kJ} / \mathrm{mol})$ of the secondary carbenium ion formed by ring opening at the more substituted carbon versus the primary carbenium ion formed by ring opening at the less substituted carbon. Furthermore, the presence of a hydroxyl group at the $\alpha$ position allows for a more facile hydride transfer to form the more stable oxocarbenium $\left(\mathrm{RCH}_{2}-\mathrm{CH}=\mathrm{O}(+) \mathrm{H}\right)$ ion intermediate. The presence of the hydroxyl substituent $\alpha$ to the $\mathrm{C}-\mathrm{O}$ bond that is activated increases the stability of the carbenium ion, such that the carbenium ion formation energy is -852 $\mathrm{kJ} / \mathrm{mol}$, while the corresponding structure for MTHF which does not have the $\alpha-\mathrm{OH}$ substituent is significantly less stable at $-743 \mathrm{~kJ} / \mathrm{mol}$.

The trend in the gas-phase carbenium ion formation energies for the acid catalyzed ring opening of THFA and MTHF was confirmed by calculations at the Re-OH acid site on the Rh-Re surface as shown in Figure 3.4. THFA adsorbs at the Re-OH acid site through the ether oxygen to form a hydrogen bond with the acid proton resulting in a calculated adsorption energy of -53 $\mathrm{kJ} / \mathrm{mol}$. The adsorbed THFA then ring opens at the more substituted carbon via the concerted protonation, ring opening and $\mathrm{H}$ transfer mechanism. In the transition state, the acid proton is 
transferred to the ether oxygen of THFA as the C-O bond of THFA is fully cleaved with the C-O distance of $2.38 \AA$. The $\mathrm{H}$ at the $\alpha-\mathrm{C}$ of the $\mathrm{C}-\mathrm{O}$ bond is also activated in the transition state and is shared between the $\alpha-\mathrm{C}$ and the $\mathrm{C}$ of the activated $\mathrm{C}-\mathrm{O}$ bond. The $\alpha-\mathrm{C}-\mathrm{H}$ bond distance increases from $1.10 \AA$ in the reactant to $1.27 \AA$ in the transition state while the distance between the $\mathrm{H}$ and the ether carbon decreases from $2.12 \AA$ in the reactant to $1.61 \AA$ in the transition state.

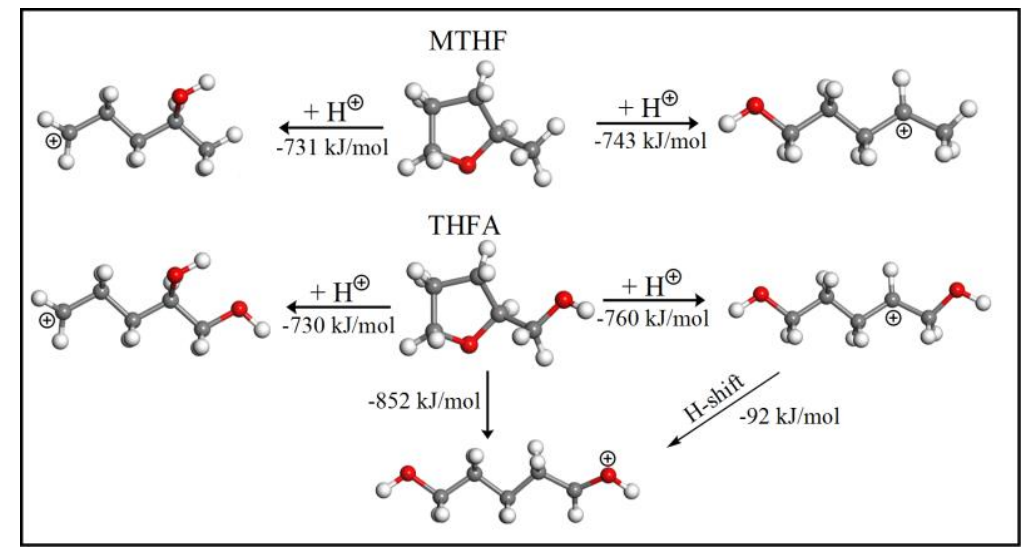

Figure 3.3 DFT calculated gas-phase carbenium ion formation energies of MTHF and THFA.

The presence of water can further stabilize the transition state as indicated by the shorter distance between the shifted $\mathrm{H}$ at the $\alpha-\mathrm{C}$ and the water oxygen in the transition state (1.70 $\AA$ ) than in the reactant $(2.40 \AA)$. The energy barrier is calculated to be $84 \mathrm{~kJ} / \mathrm{mol}$. The activation barrier for the ring opening of MTHF which does not have a $\alpha-\mathrm{OH}$ substituent is considerably higher at $118 \mathrm{~kJ} / \mathrm{mol}$. This is consistent with the trends of the gas phase carbenium ion formation energies presented above. The preferential hydrogenolysis of MTHF at the less substituted carbon along with the 30 fold decrease in activity reported experimentally on the $\mathrm{Rh}-\mathrm{Re}$ catalyst cannot be explained through acid chemistry as it would result in the formation of a highly unstable primary carbenium ion. The hydrogenolysis and ring opening of MTHF instead appears to proceed via a metal-catalyzed route similar to the ring opening of THFA over Rh surface 
shown previously in my Master's work. ${ }^{[84]}$

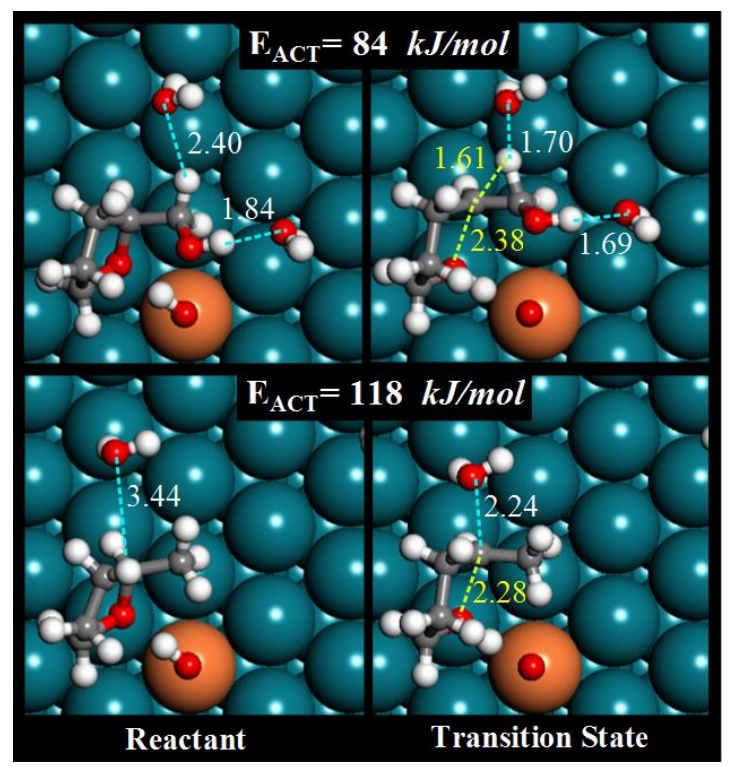

Figure 3.4 DFT-calculated reactant and transition state structures for ring opening of THFA and MTHF at the Re-OH acid site on the Rh-Re surface.

More rigorous calculations of the ring opening of THFA at the Rh-Re acid site were carried out using 40 water molecules at a density of $\sim 1 \mathrm{~g} / \mathrm{cm}^{3}$ to simulate the aqueous interface and provide a more realistic environment of the aqueous/metal interface. As shown in Figure 3.4B, the transition state of the ring opening of THFA at the more extensive aqueous solution/metal interface is very similar to that found with only 2 water molecules shown above. The energy barriers between the two are also similar, $88 \mathrm{~kJ} / \mathrm{mol}$ for the aqueous/metal interface versus 84 $\mathrm{kJ} / \mathrm{mol}$ ( 2 water molecules), indicating that the main solvent effects on the ring opening of THFA at the $\mathrm{Re}-\mathrm{OH}$ acid site come from the local water molecules and the outer shell of solvent water play little role here. 


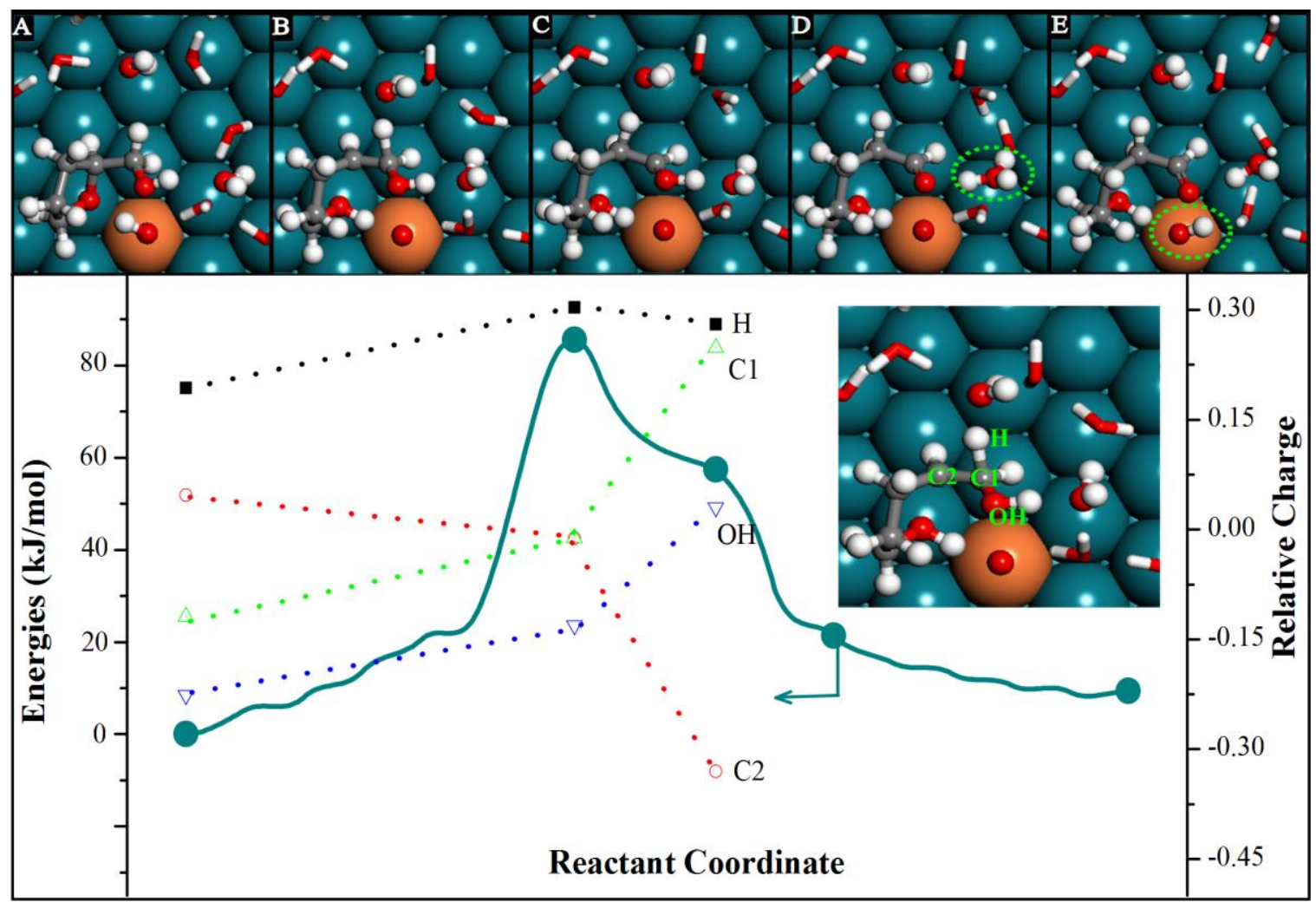

Figure 3.5 DFT calculated ring opening process of THFA at the $\mathrm{Re}-\mathrm{OH}$ acid site on $\mathrm{Rh}-\mathrm{Re}$ surface in aqueous system: A) THFA adsorption at the acid site; B)transition state of the ring opening; C); oxocarbenium ion formation; D) proton shuttling in water; E) regeneration of acid sites; and the charge analyses for the ring opening process.

A more detailed charge analysis was carried out to understand how water molecules stabilize the transition states in the ring opening of THFA. The results which are shown in Figure 3.5 indicate that the charge on $\mathrm{C} 2$ decreases from +0.04 in the reactant to -0.33 in the transition state while the charge on $\mathrm{C} 1$ increases from -0.12 in the reactant to +0.25 in the transition state indicating electron transfer from $\mathrm{C} 1$ to $\mathrm{C} 2$. The positive charge on the $\mathrm{H}$ that transfers from $\mathrm{C} 1$ to C2 also increases slightly from +0.19 in the reactant to +0.30 in the transition state. As such the hydrogen transfer is not proceed by a direct hydride transfer between $\mathrm{C} 1$ and $\mathrm{C} 2$ but instead via proton coupled electron transfer (PCET) mechanism where activation of the $\mathrm{C}-\mathrm{H}$ bond allows for an electron to transfer from $\mathrm{C} 1$ to $\mathrm{C} 2$ while the resulting proton transfers from $\mathrm{C} 1$ to $\mathrm{C} 2$. Such 
proton-coupled electron transfer processes are pervasive throughout enzymatic as well as inorganic catalytic reduction reactions. ${ }^{[121]}$ As such the hydrogen transfers as a proton and can be readily facilitated by the presence of water as water can greatly stabilize the transition state through the lone pair electrons on the oxygen. The charge on the $\alpha-\mathrm{OH}$ also changes from -0.22 in the reactant to -0.13 in the transition state and further to +0.03 in the oxocarbenium ion structure as the $\mathrm{H}$ shift is completed, which thus leads to the stabilization of the transition state by another water molecule forming hydrogen bonds with the $\alpha-\mathrm{C}-\mathrm{OH}$.

The coupled ring opening and $\mathrm{H}$ shift of THFA results in the formation of the oxocarbenium ion intermediate as shown above in Figure 3.4C. The oxocarbenium intermediate that forms, however, is not stable in aqueous solution, and will spontaneously transfer the proton on the $\mathrm{CH}=\mathrm{O}(+) \mathrm{H}$ group to the water solution with no energy barrier to form a stable intermediate $\delta$ hydroxyvaleraldehyde. The proton can readily shuttle locally in 3-dimensional hydrogen bond network of water, ${ }^{[122,123]}$ and recombine with $\mathrm{Re}-\mathrm{O}^{-}$on the $\mathrm{Rh}-\mathrm{Re}$ surface to regenerate the acid site as shown in Figure 3.4D and Figure 3.4E. The reaction energy for the ring opening of THFA to form $\delta$-hydroxyvaleraldehyde is calculated to be about $18 \mathrm{~kJ} / \mathrm{mol}$. It is worth noting that the proton shuttling in the water solvent is important for the regeneration of the acid site, which may be another reason besides the transition state stabilization by water for the experimental observed high activity of the ring opening of THFA on Rh-Re catalysts in aqueous solution as opposed to the inactivity of this reaction carried out in aprotic solvents such as 1,4-dioxane. ${ }^{[57]}$

\subsection{Hydrogenation of $\delta$-hydroxyvaleraldehyde at the metal sites}

The $\delta$-hydroxyvaleraldehyde intermediate that results from the ring opening of THFA likely binds to the Rh metal sites and can hydrogenate to form the final product 1,5-pentanediol. The 
resulting DFT calculations indicate that $\delta$-hydroxyvaleraldehyde intermediate binds at the $\mathrm{Rh}$ metal sites through the carbonyl group $(-\mathrm{C}=\mathrm{O})$. A number of studies in the literature have examined the hydrogenation of the $\mathrm{C}=\mathrm{O}$ bonds of aldehydes and ketones over metal catalysts. ${ }^{[124-127]}$ Hydrogenation proceeds via two sequential $\mathrm{H}$ addition steps to saturate the $\mathrm{C}=\mathrm{O}$ double bond to form the corresponding alcohol. As such the hydrogenation can proceed via the addition of hydrogen to the oxygen to form the hydroxyalkyl intermediate or via the addition of hydrogen to the carbon to form the alkoxide intermediate which are thus termed the hydroxyalkyl path and alkoxide path, respectively. We explored both of these paths for the hydrogenation of $\delta$ hydroxyvaleraldehyde in aqueous solution.

The addition of $\mathrm{H}$ to the carbonyl oxygen of $\delta$-hydroxyvaleraldehyde to form the hydroxyalkyl intermediate proceeds via hydrogen insertion into the Rh-O bond to form the 3center Rh-H-O transition state structure shown in Figure 3.6. The activation barrier for this path was calculated to be $84 \mathrm{~kJ} / \mathrm{mol}$. However, in aqueous solution, a more favorable path for $\mathrm{H}$ addition to the carbonyl oxygen of an aldehyde can occur via a PCET mechanism involving proton shuttling where the adsorbed $\mathrm{H}$ which acts as hydride $(\mathrm{H}-)$ transfers its electron into the metal and comes off into solution as a proton. ${ }^{[128]}$ The resulting proton can then transfer via local hydrogen bond networks to the adsorbed aldehyde where simultaneously recombines with the electron from the metal as it adds to the oxygen of the carbonyl to form the hydroxyalkyl intermediate as shown in Figure 3.6. The activation barrier for the path was calculated to be 70 $\mathrm{kJ} / \mathrm{mol}$, which is about $14 \mathrm{~kJ} / \mathrm{mol}$ lower and thus more favorable than that via the metal insertion path. This $\mathrm{H}$ addition step to form the hydroxyalkyl intermediate was calculated to be exothermic with the reaction energy of $-12 \mathrm{~kJ} / \mathrm{mol}$. The subsequent addition of hydrogen to the carbon of the hydroxyalkyl proceeds via a hydrogen insertion into the metal-C bond to form the 1,5- 
pentanediol product. ${ }^{[126,127]}$ The activation energy for this step was calculated to be $91 \mathrm{~kJ} / \mathrm{mol}$ which is significantly higher than that for $\mathrm{H}$-addition to the oxygen. The overall activation barrier for the hydrogenation of $\delta$-hydroxyvaleraldehyde to form 1,5-pentanediol through this hydroxyalkyl intermediate path is $\sim 79 \mathrm{~kJ} / \mathrm{mol}$ as shown in the energy diagram in Figure 3.8 .

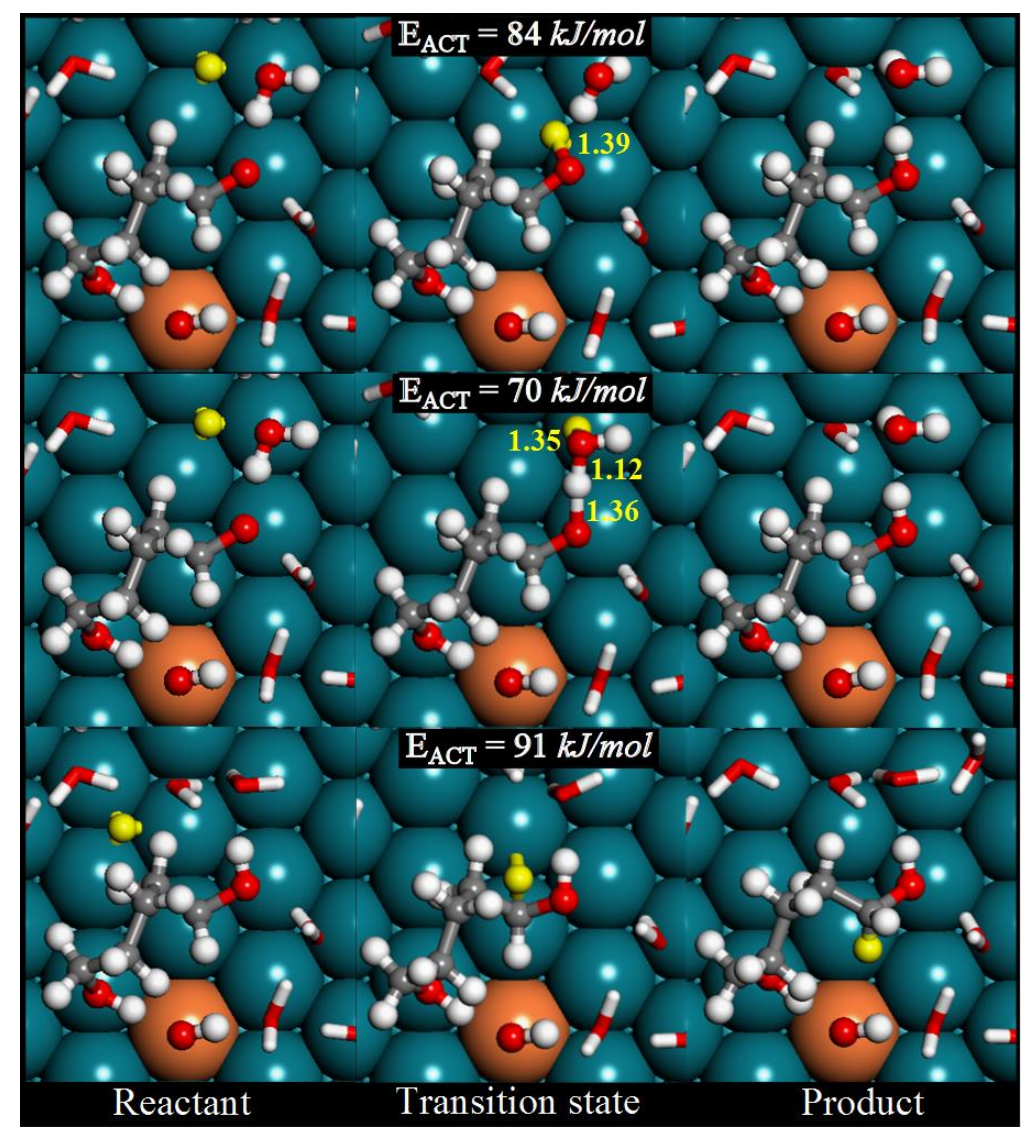

Figure 3.6 DFT-calculated reactant, transition state and product structures for the hydrogenation of $\delta$-hydroxyvaleraldehyde at the metal sites of Rh-Re surface in aqueous solution through the hydroxylalkyl path: top) first $\mathrm{H}$ addition to the carbonyl oxygen through direct metal insertion mechanism; center) first $\mathrm{H}$ addition to the carbonyl oxygen via PCET mechanism to form a hydroxylalkyl intermediate; bottom) second $\mathrm{H}$ addition to the carbon of the hydroxyalkyl intermediate to form 1,5-pentanediol.

The hydrogenation of $\delta$-hydroxyvaleraldehyde can also proceed via the initial addition of hydrogen to the $\mathrm{C}$ of the carbonyl to form the alkoxide intermediate. The activation barrier and 
the overall reaction energy for the addition of $\mathrm{H}$ to the carbon of the carbonyl group to form an alkoxide intermediate were calculated to be 81 and $20 \mathrm{~kJ} / \mathrm{mol}$, respectively. The activation energy for the subsequent addition of $\mathrm{H}$ to the alkoxide intermediate to form 1,5-pentanediol was calculated to be $80 \mathrm{~kJ} / \mathrm{mol}$. Thus, the overall apparent barrier for the hydrogenation of $\delta$ hydroxyvaleraldehyde to form 1,5-pentanediol through the alkoxide path was calculated to be $104 \mathrm{~kJ} / \mathrm{mol}$ as shown in the energy diagram in Figure 3.8. The lower overall energy barrier that results from the hydroxyalkyl path than that for the alkoxide path suggests that the former is more favorable for the hydrogenation of $\delta$-hydroxyvaleraldehyde at the metal sites of Rh-Re catalyst in aqueous solution.

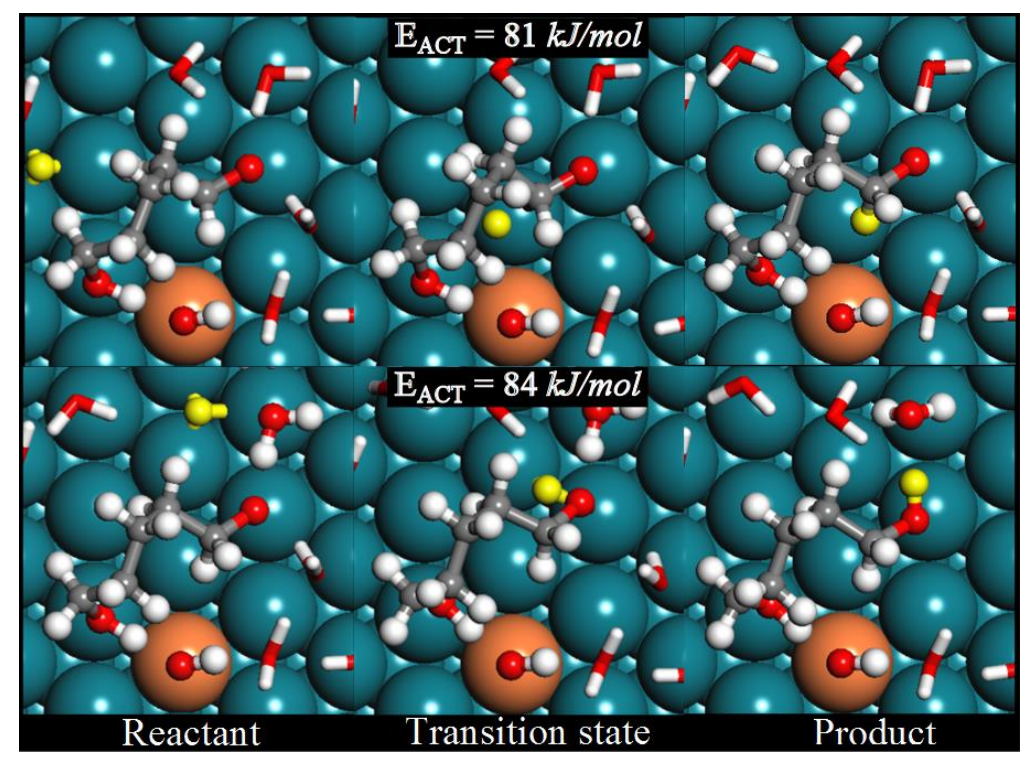

Figure 3.7 DFT-calculated reactant, transition state and product structures for the hydrogenation of $\delta$-hydroxyvaleraldehyde at the metal sites of Rh-Re surface in aqueous solution through the alkoxide path: top) first $\mathrm{H}$ addition to the carbonyl oxygen to form an alkoxide intermediate; bottom) second $\mathrm{H}$ addition to the oxygen of the alkoxide intermediate to form 1,5-pentanediol. 


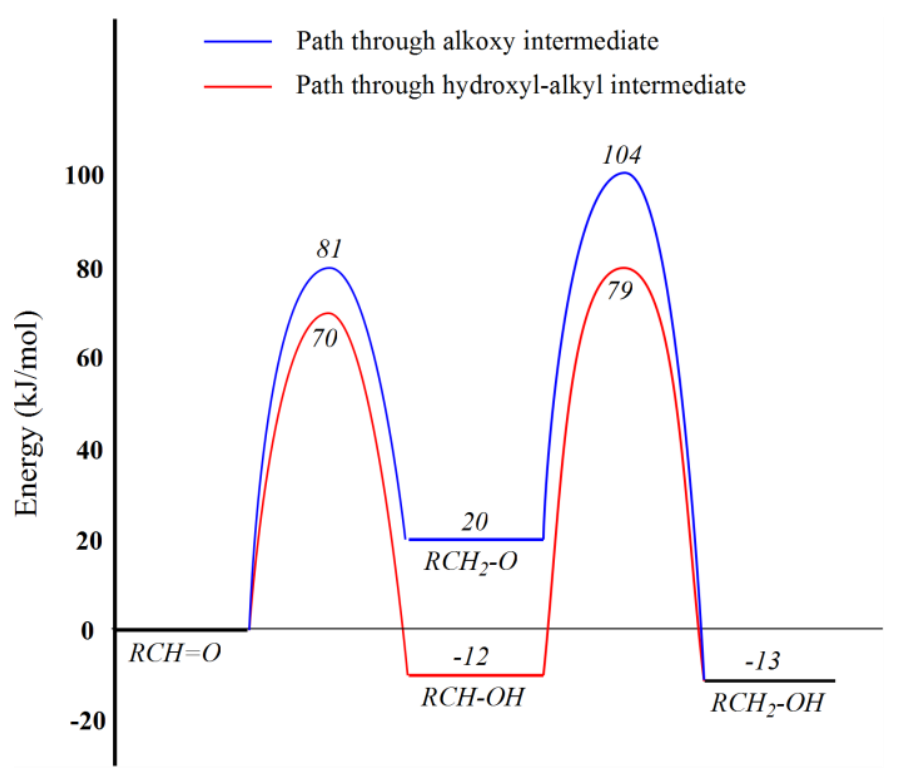

Figure 3.8 Potential energy diagrams for the two different paths for the hydrogenation of $\delta$ hydroxyvaleraldehyde at the metal sites on the Rh-Re surface in aqueous solution: (red) hydroxylalkyl path and (blue) alkoxide path.

\subsection{Kinetics analysis for the hydrogenolysis of THFA}

By combining the results for the ring opening of THFA at the Re-OH site along with the results for the hydrogenation on the Rh sites on Rh-Re surface, we can construct the complete reaction energy diagram shown in Figure 3.9 for the hydrogenolysis of THFA to form 1,5pentanediol over the bifunctional Rh-Re catalyst in aqueous solution. Both the ring opening and the second $\mathrm{H}$ addition steps are the highest in energy along the path thus suggesting that one or both of these steps may be rate limiting. We note, however, that the well-dispersed single $\mathrm{Re}-\mathrm{OH}$ acid sites embedded in the $\mathrm{Rh}(111)$ surface model that was used herein is rather simple, and may underestimate the acidity of the real acid sites on the Rh-Re catalyst. The presence of stronger acid would significantly lower the reported activation barrier as will be shown later. In addition, experimental kinetic studies showed that the rate of the hydrogenolysis of THFA over the Rh-Re catalyst has a first order dependence on the hydrogen pressure. ${ }^{[54,63,72]}$ The experimental 
observations along with the initial DFT results indicate that the acid catalyzed C-O activation is quasi-equilibrated and that the rate of THFA hydrogenolysis over the Rh-Re catalyst is controlled by the second hydrogenation step.

The possible elementary reactions in the hydrogenolysis of THFA over the bifunctional RhRe catalyst were presented in Figure 3.10. The first three steps which involve the adsorption of THFA, the ring opening of THFA and the regeneration of the Brønsted acid site all occur at the acid sites. The last 7 steps occur including the adsorption of the ring opened $\delta$ hydroxyvaleraldehyde intermediate, the dissociative adsorption of $\mathrm{H}_{2}$, the hydrogenation of the adsorbed $\delta$-hydroxyvaleraldehyde and the desorption of the 1,5-pentanediol product all occur on the metal sites. It is noted that the reactant THFA and the water in the solution phase can both adsorb and compete for the metal sites.

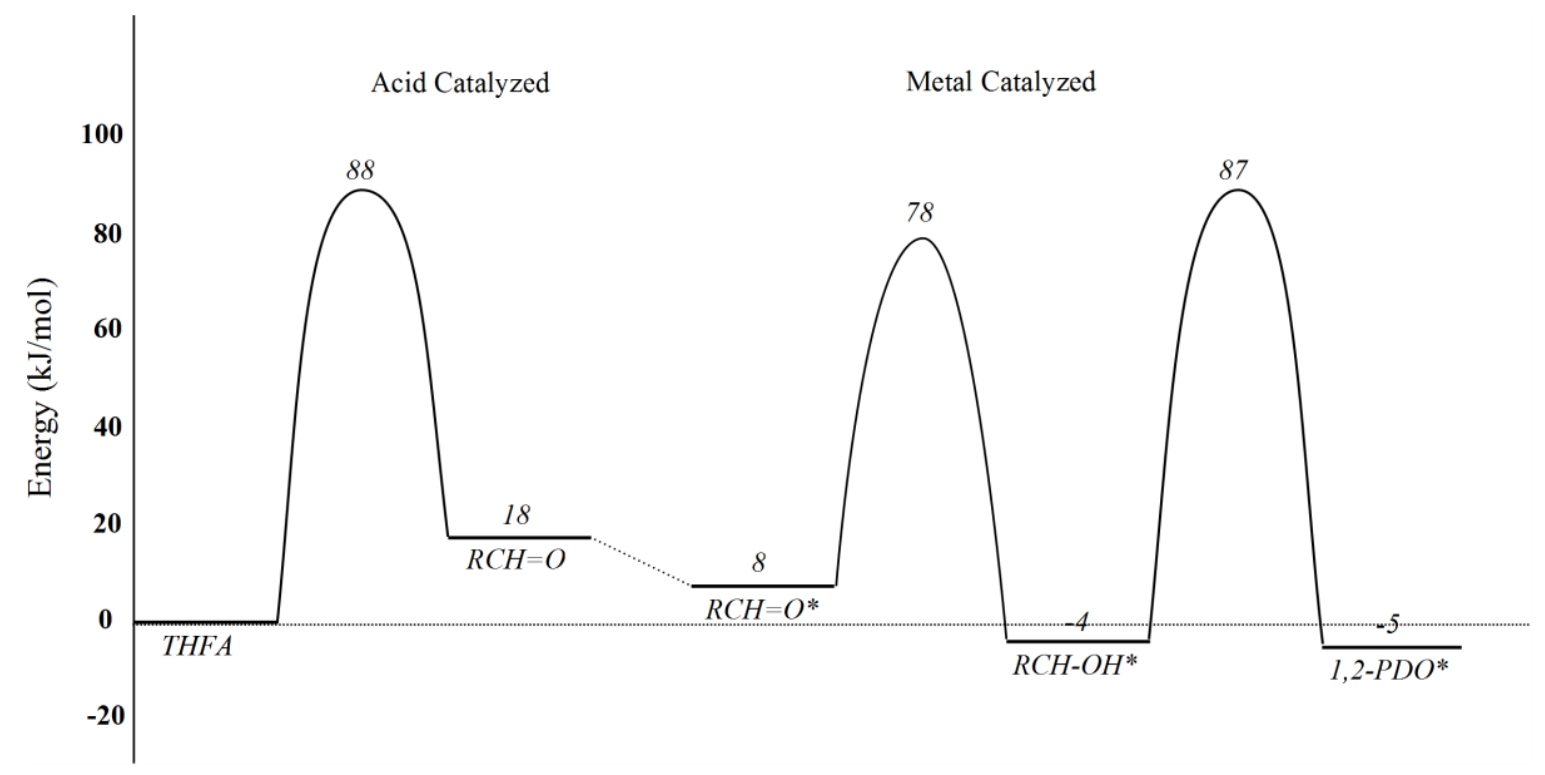

Figure 3.9 Potential energy profiles for the hydrogenolysis of THFA on the bifunctional Rh-Re surface in aqueous solution. 


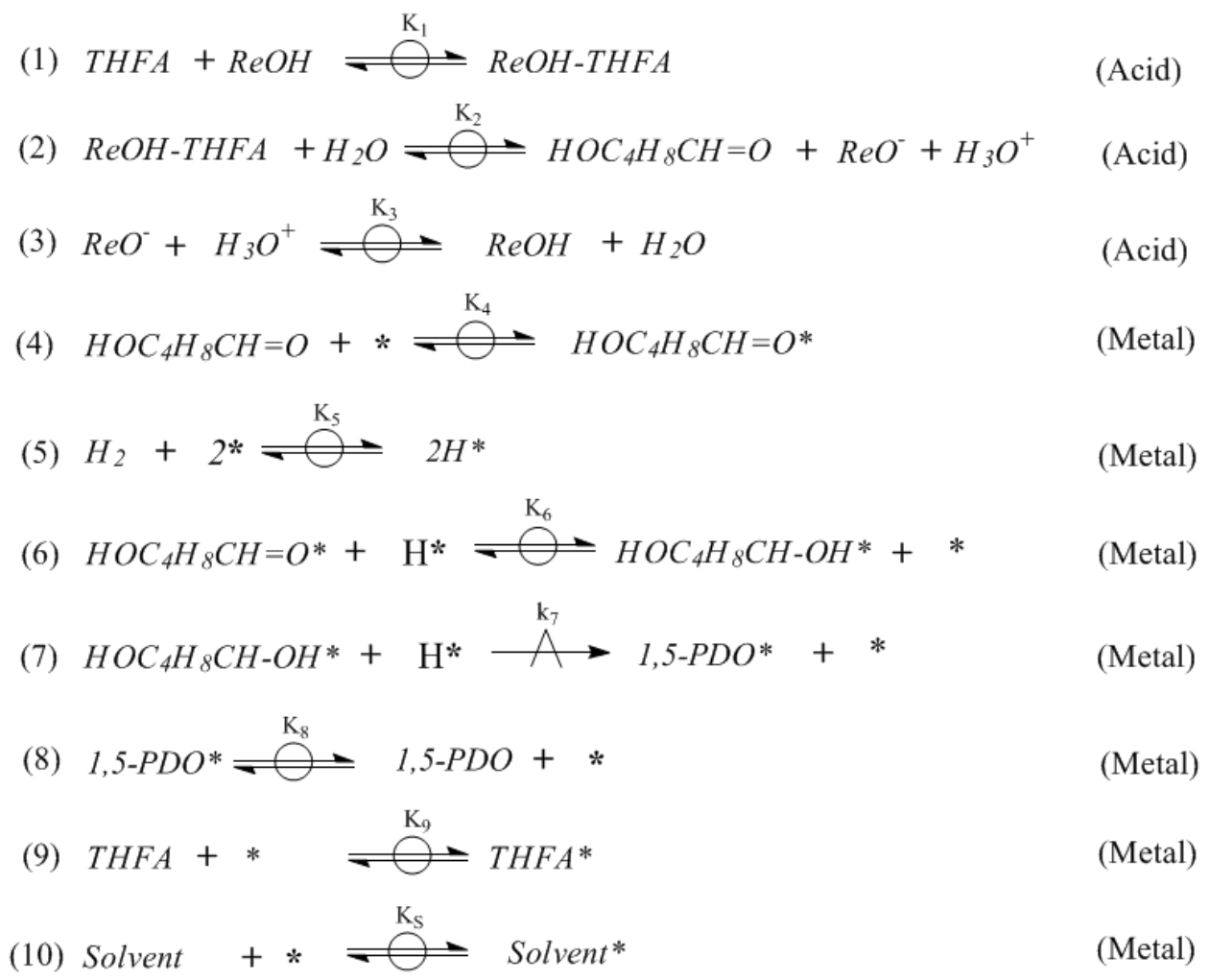

Figure 3.10 Possible elementary steps that occur in the hydrogenolysis process of THFA on RhRe catalyst in aqueous solution. The solid acid sites on Re are denoted as $\mathrm{ReOH}$ whereas the metal sites involved in the hydrogenation are listed as *.

As discussed above, the DFT-results suggest that the second $\mathrm{H}$ addition to the $\mathrm{C}$ of the hydroxyalkyl intermediate to form the 1-5 pentanediol product may be the rate limiting step and that all of the other reactions are quasi-equilibrated. By making these assumptions we can derive the rate expression presented in Equation 3.6 for the hydrogenolysis of THFA over Rh-Re. The different terms in the denominator of Equation 3.6 correspond to the coverage of different adsorbed intermediates on the metal surface. Under the reaction conditions, the water solution is present in the greatest amounts and is strongly held to the surface and as a result, it will likely cover the surface. Hence, the rate expression of the hydrogenolysis of THFA on Rh-Re catalyst described in Equation (3.6) can be simplified as shown in Equation (3.7). From the rate expression in Equation (3.7), we can see that the rate of THFA hydrogenolysis is first order of the 
hydrogen pressure, first order in the concentration of THFA, which is consistent with the experimental observations by Dumesic et al. ${ }^{[72]}$ The first order THFA kinetic behavior reported herein and by Dumesic et al., however, is higher than that observed by Tomishige et al. ${ }^{[54]}$ who report a THFA reaction order of $\sim 0.2$ It is noted that the concentration of the reactant THFA in the kinetics study by Tomishige et al. ${ }^{[54]}$ is $\sim 10$ times higher than that in the kinetics study by Dumesic et al. ${ }^{[72]}$ At higher THFA concentrations, the terms involving $C_{\mathrm{THFA}}$ in the denominator of the rate expression Equation (3.6) are no longer negligible and thus act to decrease the dependency of the rate on the concentration of THFA, which would explain the weaker THFA dependencies on the rate observations by Tomishige et al. ${ }^{[54]}$

$$
\begin{gathered}
r=\frac{k_{7} K_{1} K_{2} K_{3} K_{4} K_{5} K_{6} p_{H_{2}} C_{\text {THFA }}}{\left(1+K_{1} K_{2} K_{3} K_{4} C_{\text {THFA }}+K_{5}^{1 / 2} p_{H_{2}}^{1 / 2}+K_{1} K_{2} K_{3} K_{4} K_{5}^{1 / 2} K_{6} P_{H_{2}}^{1 / 2} C_{\text {THFA }}+\frac{C_{1.5 P D O}}{K_{8}}+K_{9} C_{\text {THFA }}+K_{S} C_{S}\right)^{2}} \\
r=\frac{k_{7} K_{1} K_{2} K_{3} K_{4} K_{5} K_{6} p_{H_{2}} C_{\text {THFA }}}{\left(K_{S} C_{S}\right)^{2}}
\end{gathered}
$$

\subsection{Ring Opening of THFA at Different Acid Sites}

The model that we used above to examine the acid site on the Rh-Re surface was rather simple. Herein we expand these ideas to other possible acid sites present on the Rh-Re catalyst. The experiments were carried out over Rh-Re metal particles of $\sim 2.2-2.3 \mathrm{~nm} .{ }^{[72]}$ We therefore extended our initial calculations carried out over $\mathrm{Rh}(111)$ surfaces to the $\mathrm{Rh}_{201}$ cubo-octahedral cluster to establish the influence from the more coordinatively-unsaturated sites. As shown in Fig. 3.11, the hydroxyl group that form as a result of the dissociation of water can sit at the terrace, edge or the corner Re site of the Rh-Re particle and result in three different acid sites. 
The deprotonation energy (DPE) of these sites were calculated and listed in Table 3.1. We see that the DPE for all three sites are similar at $\sim 1145 \mathrm{~kJ} / \mathrm{mol}$, indicating the same acid strength of these three sites. This seemed rather surprising as the metal atom coordination number decreased from 9,8 to 7 in moving from the terrace to the edge to the corner site.
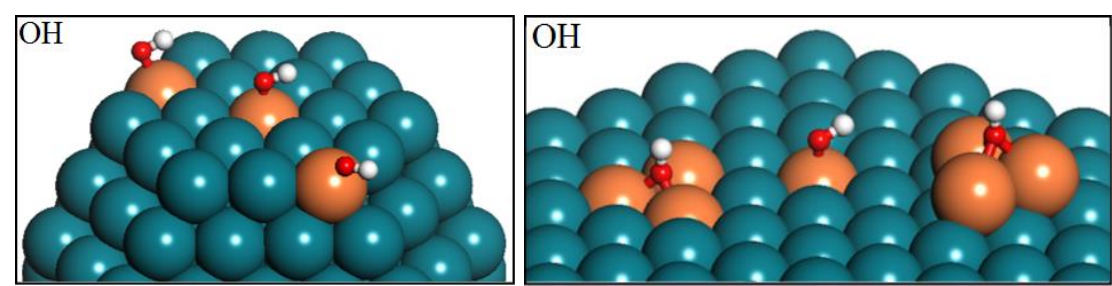

Figure 3.11 Different models of the possible acid sites on the Rh-Re catalyst: left) Re-OH located at different positions of the Rh cluster (terrace, edge and corner); right) $\mathrm{OH}$ binding 3fold to three adjacent Re atoms that can be in the Rh surface or above the Rh surface.

Table 3.1 DFT-calculated DPE of different Re-OH acid sites on Rh-Re particle, the adsorption energies of THFA and the activation barriers for its ring opening at these different acid sites, together with the binding energies of $\mathrm{O}^{*}$ and $\mathrm{OH}^{*}$ at the Re sites related to the acidity of Re-OH. The units are in $\mathrm{kJ} / \mathrm{mol}$.

\begin{tabular}{lccccc}
\hline & $\begin{array}{c}\mathrm{Re}_{1} \mathrm{OH} \\
\text { (terrace) }\end{array}$ & $\begin{array}{c}\mathrm{Re}_{1} \mathrm{OH} \\
(\text { edge })\end{array}$ & $\begin{array}{c}\mathrm{Re}_{1} \mathrm{OH} \\
\text { (corner) }\end{array}$ & $\mathrm{Re}_{3} \mathrm{OH}-\mathrm{IN}$ & $\mathrm{Re}_{3} \mathrm{OH}-\mathrm{OV}$ \\
\hline$D P E$ & 1145 & 1146 & 1145 & 1060 & 1112 \\
$\Delta E_{\text {ads }}(\mathrm{OH})$ at Re & -314 & -385 & -450 & -298 & -369 \\
$\Delta E_{\text {ads }}(\mathrm{O})$ at $\mathrm{Re}$ & -549 & -617 & -682 & -613 & -634 \\
$\Delta E_{\text {ads }}(\mathrm{O})-\Delta E_{\text {ads }}(\mathrm{OH})$ & -235 & -232 & -232 & -315 & -265 \\
\hline$\Delta E_{\text {ads }}($ THFA $)$ at $\mathrm{Re}-\mathrm{OH}$ & -53 & -54 & -55 & -93 & -67 \\
$\Delta E_{A C T}(\mathrm{THFA})$ at $\mathrm{Re}-\mathrm{OH}$ & 88 & 38 & 56 & 50 & 72 \\
\hline
\end{tabular}

In previous work, ${ }^{[129]}$ we showed that the DPE of the hydroxyl group bound at the Rh-Re and Pt-Re alloys is not directly linearly correlated with the $\mathrm{O}^{*}$ binding energy but instead with 
the difference between the binding energies of $\mathrm{O}^{*}$ and $\mathrm{OH}^{*}$ at the alloy site. While the $\mathrm{O}^{*}$ binding energy increases as we move from terrace to edge to corner as is shown in Table 3.1, the $\mathrm{OH}^{*}$ binding energies increase by essentially the same amount which results in values for $\left[\Delta E_{a d s}\right.$ (O) $\left.-\Delta E_{a d s}(\mathrm{OH})\right]$ that are nearly the same at these three sites, and thus similar deprotonation energies.

The ring opening of THFA at these three Re-OH acid sites was subsequently examined to see if there were any differences in the activation barriers. The results are presented in Figure 3.12. As shown above, the solvent stabilization of the transition state of the ring opening of THFA predominantly results from the presence of two local water molecules. For simplicity, we used only two water molecules to solvate the ring opening of THFA over the Re-promoted $\mathrm{Rh}_{201}$ cluster in solution. The results show similar binding modes and adsorption strengths for THFA and structures for the ring opening transition states at the terrace, edge and corner $\mathrm{Re}-\mathrm{OH}$ acid sites on the Rh-Re particle as shown in Figure 3.12. The binding energy of THFA at the terrace Re-OH site was calculated to be $-50 \mathrm{~kJ} / \mathrm{mol}$, which is very similar to that found at the $\mathrm{Re}-\mathrm{OH}$ sites embedded into the $\mathrm{Rh}(111)$ surface $(-53 \mathrm{~kJ} / \mathrm{mol})$. The activation energy for the ring opening of THFA at the terrace $\mathrm{Re}-\mathrm{OH}$ site on the $\mathrm{Re}-\mathrm{OH}$ promoted $\mathrm{Rh}_{201}$ cluster was calculated to be 88 $\mathrm{kJ} / \mathrm{mol}$, which is also in very good agreement with the results for the Re-OH sites in the $\mathrm{Rh}(111)$ surface $(84 \mathrm{~kJ} / \mathrm{mol})$. This suggests that the much simpler surface Re-OH acid site model can provide a reasonably good representation of the (111) terrace $\mathrm{Re}-\mathrm{OH}$ acid sites on the 201-atom particle. Similarly, the ring opening of THFA at the edge and corner acid sites are also shown in Figure 3.12 and the energetics are listed in Table 3.1. We see that the binding energies of THFA at the edge and corner Re-OH sites are similar to that at the terrace site $(-53 \sim-54 \mathrm{~kJ} / \mathrm{mol})$, which is reasonable as the binding energy of an adsorbate at the solid acid is reported to be related to 
the acidity of the solid acid ${ }^{[120]}$ and the acidity of these three Re-OH sites is similar as shown above.

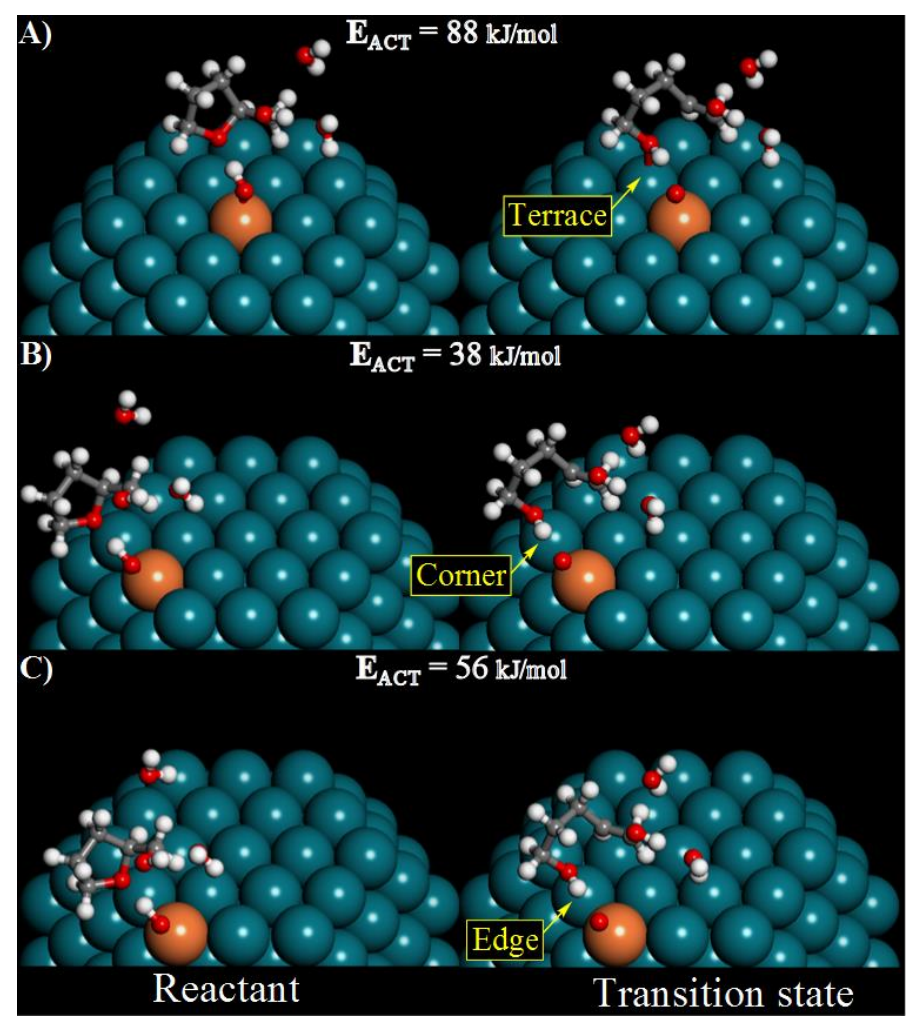

Figure 3.12 DFT-calculated reactant and transition state structures for ring opening of THFA at different Re-OH acid sites on the Rh-Re particle: (A) (111) terrace; (B) edge; (C) corner.

The activation energies for the ring opening of THFA at the edge $\mathrm{Re}-\mathrm{OH}$ site $(38 \mathrm{~kJ} / \mathrm{mol})$ and the corner Re-OH site $(56 \mathrm{~kJ} / \mathrm{mol})$, however, were calculated to be much lower than at the terrace $\mathrm{Re}-\mathrm{OH}$ site $(88 \mathrm{~kJ} / \mathrm{mol})$. As shown in Figure 3.12, after the proton-transfer from the Re$\mathrm{OH}$ acid site to the ether oxygen of THFA and ring opening, an alcohol hydroxyl group is formed in the transition state, which binds to a neighboring $\mathrm{Rh}$ atom that is adjacent to the $\mathrm{Re}-\mathrm{OH}$ site. When THFA ring opens at the terrace $\mathrm{Re}-\mathrm{OH}$ site, the hydroxyl group in the transition state binds to a neighboring terrace $\mathrm{Rh}$ atom, while when THFA ring opens at the edge or corner acid sites, the hydroxyl group binds to a corner $\mathrm{Rh}$ atom or an edge $\mathrm{Rh}$ atom respectively. The binding 
energies of the hydroxyl oxygen in the ring-opened transition state at the different vicinal $\mathrm{Rh}$ atom sites (terrace, edge or corner) can be linearly related to the changes in the reported activation barriers for ring opening of THFA at these three $\mathrm{Re}-\mathrm{OH}$ acid sites even though they have the same acidity.

The simplest alcohol methanol was used to estimate the binding energies of an alcohol hydroxyl group at different neighboring metal atoms of these three Re-OH acid sites. As shown in Fig. 3.13, the activation energies for the ring opening of THFA at the corner, edge and terrace Re-OH acid sites can be linearly correlated to the binding energies of methanol at the neighboring $\mathrm{Rh}$ atoms of these $\mathrm{Re}-\mathrm{OH}$ acid sites and the slope is about 1.0, indicating that the different activation energies for the ring opening of THFA at these terrace, edge and corner Re$\mathrm{OH}$ acid sites which have similar acidities is the direct result of the different binding energies of the hydroxyl group in the transition state at the neighboring metal atoms of these acid sites. The ring opening of THFA has the lowest activation barrier at the $\mathrm{Re}-\mathrm{OH}$ acid sites that reside at the edges of the $\mathrm{Rh}_{201}$ cluster. This is due to the strongest binding and stabilization of the $\mathrm{OH}$ group in the transition state to the vicinal Rh corner site.

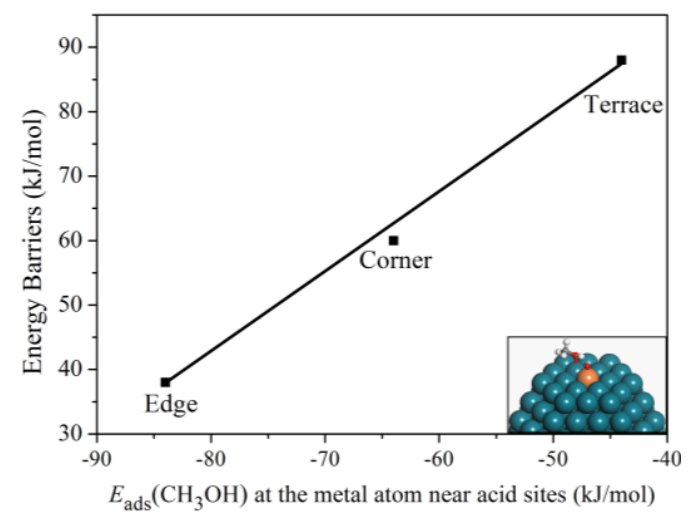

Figure 3.13 Correlation of the activation energies for the ring opening of THFA at three different acid sites with $\mathrm{Re}-\mathrm{OH}$ located at the terrace, edge and corner sites of $\mathrm{Rh}_{201}$ particle versus the binding energies of methanol at the vicinal $\mathrm{Rh}$ atoms of the acid sites. 
To this point we have only considered isolated Re sites that sit within the surface of the RhRe particle. The active sites could also be comprised of larger clusters of Re that sit either within or upon the $\mathrm{Rh}$ cluster. We first examine the acidity of $\mathrm{OH}$ bound the 3 -fold site of 3 or more $\mathrm{Re}$ atom clusters within the $\mathrm{Rh}$ surface. As shown in Figure 3.11, the $D P E$ of this $\mathrm{Re}_{3} \mathrm{OH}$ site embedded into the $\mathrm{Rh}$ surface $\left(\mathrm{Re}_{3} \mathrm{OH} \_\mathrm{IN}\right)$ was calculated to be $1060 \mathrm{~kJ} / \mathrm{mol}$, indicating that it is more acidic than the single $\mathrm{Re}-\mathrm{OH}$ acid sites shown above.

The active Re sites can also sit on top of the Rh surface rather than within the surface. This is consistent with recent suggestions in the literature which suggest such supported clusters. ${ }^{[64]}$ We examined herein the 3 Re atom cluster bound to adjacent three-fold sites on the Rh surface as a simple model as is shown in Figure 3.11. The $D P E$ of this acid site $\left(\mathrm{Re}_{3} \mathrm{OH} \_\mathrm{OV}\right)$ was calculated to be $1112 \mathrm{~kJ} / \mathrm{mol}$, indicating it is more acidic than the Re-OH acid sites that reside in the surface $(1145 \mathrm{~kJ} / \mathrm{mol})$, but less acidic than the $\mathrm{Re}_{3} \mathrm{OH} \_\mathrm{IN}$ acid site $(1060 \mathrm{~kJ} / \mathrm{mol})$. The lower $D P E$ of these two acid sites $\left(\mathrm{Re}_{3} \mathrm{OH}_{-} \mathrm{IN}\right.$ and $\left.\mathrm{Re}_{3} \mathrm{OH} \_\mathrm{OV}\right)$ is due to the relatively stronger binding of $\mathrm{O}$ but weaker binding of $\mathrm{OH}$ at the 3-fold metal sites than the atop metal sites, which leads to the more negative value of $\left[\Delta E_{a d s}(\mathrm{O})-\Delta E_{a d s}(\mathrm{OH})\right]$ as shown in Table 3.1.

The adsorption and ring opening of THFA at the more acidic $\mathrm{Re}_{3} \mathrm{OH}$ IN and $\mathrm{Re}_{3} \mathrm{OH} \mathrm{OHV}_{-}$ acid sites were examined in detail. The binding energy of THFA at the $\mathrm{Re}_{3} \mathrm{OH}$ IN and $\mathrm{Re}_{3} \mathrm{OH} \_\mathrm{OV}$ acid sites were calculated to be $-93 \mathrm{~kJ} / \mathrm{mol}$ and $-67 \mathrm{~kJ} / \mathrm{mol}$ respectively, which are appreciably stronger than the adsorption of THFA at the terrace Re-OH site presented earlier $(-55$ $\mathrm{kJ} / \mathrm{mol}$ ). The activation barriers for the ring opening of THFA at the $\mathrm{Re}_{3} \mathrm{OH} \_\mathrm{IN}$ and $\mathrm{Re}_{3} \mathrm{OH} \mathrm{OV}_{-}$ acid sites were calculated to be $50 \mathrm{~kJ} / \mathrm{mol}$ and $72 \mathrm{~kJ} / \mathrm{mol}$ respectively, which are lower than that at the terrace Re-OH acid site. The binding energies for THFA as well as the activation barriers for THFA ring opening were found to be linearly correlated to the $D P E$ of the different acid sites 
examined as is shown in Figure 3.14, which thus directly relates the change in solid acid strength $(D P E)$ to the ring opening activity.
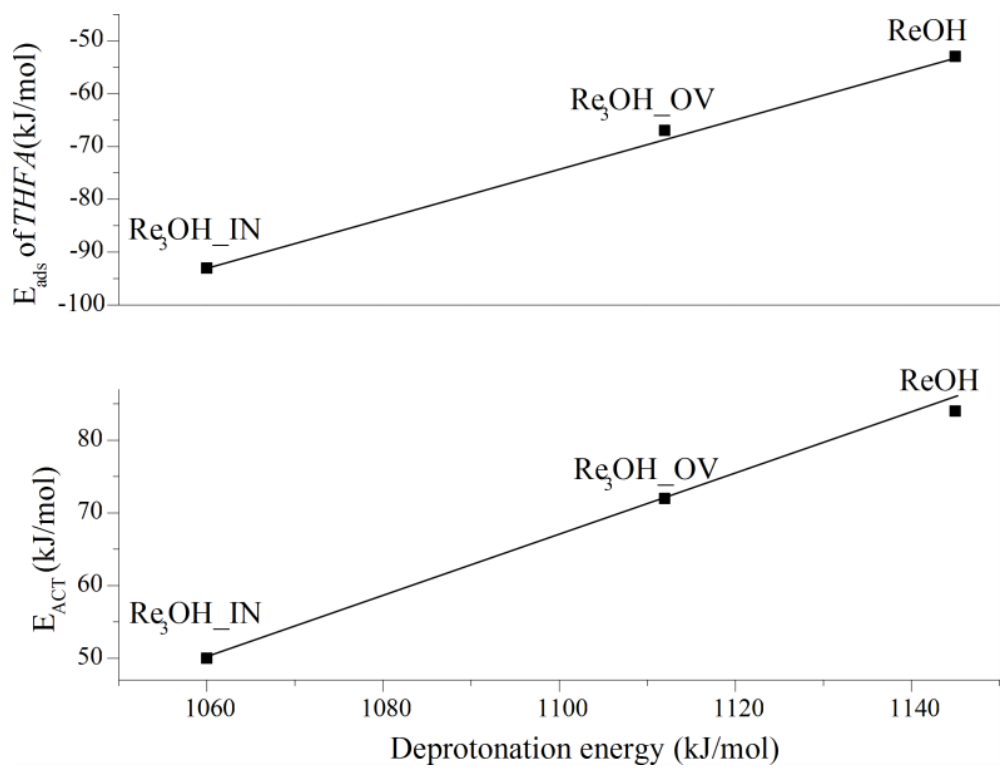

Figure 3.14 Correlation of the adsorption energies of THFA and activation energies for its ring opening at the three different acid sites ( $\mathrm{ReOH}, \mathrm{Re}_{3} \mathrm{OH}-\mathrm{IN}$ and $\mathrm{Re}_{3} \mathrm{OH}$-OV) versus the deprotonation energies of the acid sites.

We have previously ${ }^{[129]}$ examined the influence of the metal cluster and the alloy promoter on the acid site strength for a series of different bimetallic alloys comprised of a reducible metal such as Rh, Pt, Ir and Pd that carry out hydrogenation reactions and an oxophilic promoter metal such as $\mathrm{Re}, \mathrm{Mo}, \mathrm{W}$ and $\mathrm{Ru}$ which are partially oxidized under reaction condition and result in strongly acidic $\mathrm{OH}$ groups. We extend these efforts here by calculating the activation barriers for the ring opening of THFA at the M-OH acid sites on these different alloy surfaces. We show that there is a direct linear relationship between the adsorption energies of THFA and the ring opening activation barriers of THFA at these different acid sites. The adsorption energies as well as the activation barriers were also both found to be correlated with the deprotonation energies of 
the acid sites as is shown in Figure 3.15.

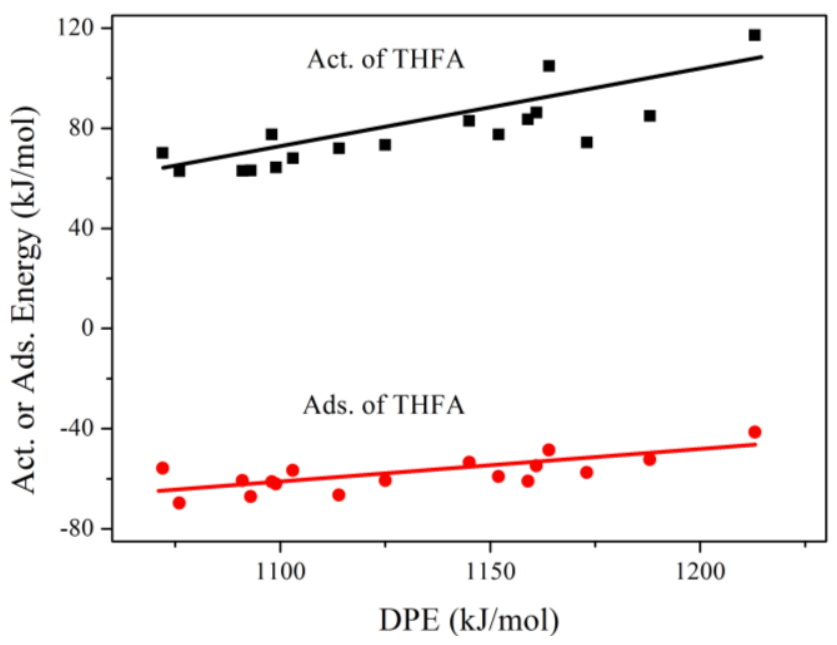

Figure 3.15 The adsorption energies of THFA and the energy barriers for its ring-opening at different $\mathrm{MOH}$ sites $(\mathrm{M}=\mathrm{Ru}, \mathrm{Mo}, \mathrm{Re}, \mathrm{W})$ embedded in $\mathrm{Ru}, \mathrm{Rh}, \mathrm{Pt}$ and $\mathrm{Au}$ (111) metal surfaces plotted against the DPE of the $\mathrm{MOH}$ sites.

The deprotonation energy for the formation of $\mathrm{Mo}-\mathrm{OH}$ at a Mo site at a terrace site within the surface of the Rh-Mo alloy particle, for example, was calculated to be $1162 \mathrm{~kJ} / \mathrm{mol}$, thus indicating the acid strength of this $\mathrm{Mo}-\mathrm{OH}$ is weaker than the acid strength of the $\mathrm{Re}-\mathrm{OH}$ site within the surface of the Rh-Re alloy particle that was reported earlier. The weaker Mo-OH acid site strength weakens the binding of THFA at this terrace Mo-OH site which is consistent with the decrease in the THFA adsorption energy from $-53 \mathrm{~kJ} / \mathrm{mol}$ at the terrace Re-OH acid site to $48 \mathrm{~kJ} / \mathrm{mol}$ for the Mo-OH acid site. The decreased acidity along with the decrease in the adsorption energy of THFA ultimately results in an increase in the activation barrier of $88 \mathrm{~kJ} / \mathrm{mol}$ for the ring opening of THFA at the Rh-Re terrace site to $95 \mathrm{~kJ} / \mathrm{mol}$ for the ring opening of THFA at the Rh-Mo site.

The general linear relation between the adsorption energies of THFA at different acid sites and the deprotonation energies of the acid sites indicates that even though the acid catalyzed ring 
opening step may not be the rate limiting step as we showed earlier in section 3.4, the acidity of the acid sites can still affect the reaction rate in the hydrogenolysis of THFA by changing the value of equilibrium constant $K_{l}$ in Equation (3.6) and Equation (3.7). Lower reaction rate in the hydrogenolysis of THFA over the Rh-Mo catalysts than over the Rh-Re catalysts has been confirmed by experiments. ${ }^{[72]}$

\subsection{Insights into other cyclic ethers and polyols}

The ideas concerning the bifunctional nature of the Rh-Re catalysts and the types of potential active sites in the hydrogenolysis of THFA over Rh-Re catalysts can be extended to other cyclic ethers and polyols. The dehydration of the polyol 1,2-propanediol (discussed previously in Chapter 2 over the monometallic Rh surface) at both the more substituted central $\mathrm{C}-\mathrm{OH}$ and the less substituted terminal $\mathrm{C}-\mathrm{OH}$ bonds and the mono-functional 2-propanol at the acid sites of Rh-Re surface are compared in Figure 3.16. Similar to the ring opening of THFA, the dehydration of 1,2-propanediol at both the less substituted terminal $\mathrm{C}-\mathrm{OH}$ and the more substituted center $\mathrm{C}-\mathrm{OH}$ proceed via the concerted protonation of the alcohol, $\mathrm{C}-\mathrm{O}$ bond scission and a $\mathrm{H}$ transfer step which proceeds via proton coupled electron transfer and is facilitated by water which stabilizes the transition state. The energy barrier for the $\mathrm{C}-\mathrm{O}$ activation of 1,2propanediol at the tertiary center carbon was calculated to be $95 \mathrm{~kJ} / \mathrm{mol}$, which is $11 \mathrm{~kJ} / \mathrm{mol}$ higher than THFA. This is in consistence with the trends reported from the gas phase carbenium ion formation energies for 1,2-propanediol and THFA. ${ }^{[72]}$ The barrier for the activation of the CO bond of 1,2-propanediol at the less substituted terminal carbon was calculated to be 108 $\mathrm{kJ} / \mathrm{mol}$, which is about $13 \mathrm{~kJ} / \mathrm{mol}$ higher than the barrier to activate $\mathrm{C}-\mathrm{O}$ bond at the more substituted carbon center. This is in agreement with the experiments that a higher selectivity in 
activating the more substituted central $\mathrm{C}-\mathrm{OH}$ bond of 1,2-propanediol thus resulting in the formation of $87.8 \%$ 1-pentanol. ${ }^{[18]}$ The activation barrier for the dehydration of the monofunctional 2-propanol which does not have a neighboring $\alpha-\mathrm{C}-\mathrm{OH}$ to stabilize the transition state was calculated to be $120 \mathrm{~kJ} / \mathrm{mol}$, which is $\sim 25 \mathrm{~kJ} / \mathrm{mol}$ higher than that for the dehydration of 1,2propanediol at the center carbon, which contains an $\mathrm{OH}$ substituent at the $\alpha$-C. This is in agreement with the experimental results that show a rather low hydrogenolysis activity for the polyols and alcohols that do not contain $\mathrm{OH}$ substituents that sit $\alpha$ to the $\mathrm{C}-\mathrm{OH}$ bond that is cleaved over the Rh-Re catalytic surfaces. ${ }^{[72]}$

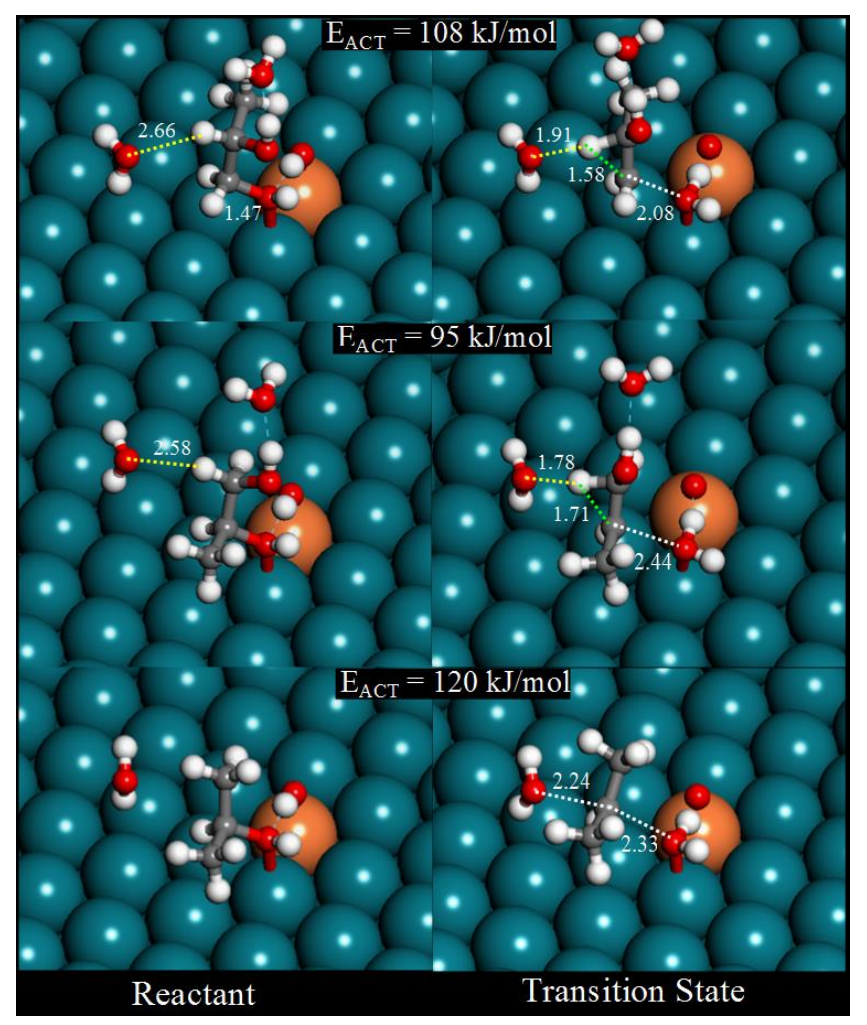

Figure 3.16 DFT-calculated reactant and transition state structures for the C-O bond activation of 1,2-propanediol at the less substituted terminal carbon (top), at the more substituted center carbon (middle) and the $\mathrm{C}-\mathrm{O}$ bond activation of mono-functional alcohol 2-propanol at the Re$\mathrm{OH}$ acid sites on $\mathrm{Rh}$-Re surface (bottom). 
Other cyclic ethers and polyols were also examined. As shown in Figure 3.17, the barriers for the activation of the $\mathrm{C}-\mathrm{O}$ activation for different cyclic ethers and polyols at the $\mathrm{Re}-\mathrm{OH}$ acid site within the Rh-Re alloy surface can be linearly correlated to their corresponding gas phase carbenium ion formation energies, which confirms the relationship presented in Equation (3.5) that was derived from a Born-Haber cycle analysis. This suggests that the reactivity of different oxygenates over the Rh-Re catalyst can be estimated by using only simple gas-phase carbenium ion formation energy calculations.

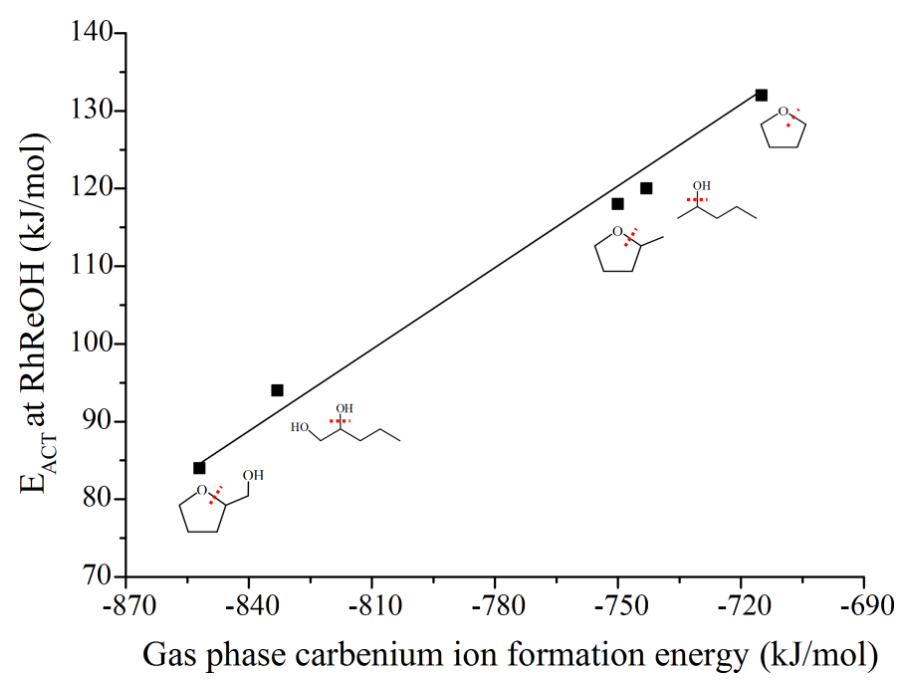

Figure 3.17 The correlation of the activation barriers for $\mathrm{C}-\mathrm{O}$ bond activation of various cyclic ethers and linear polyols or mono-functional alcohols at the Re-OH acid sites on the Rh-Re surface versus their corresponding gas phase carbenium ion formation energies.

\section{Conclusions}

In this chapter, the mechanism for the hydrogenolysis of cyclic ether THFA at the bifunctional Rh-Re catalyst was systematically investigated with the modeled Re-OH acid sites. The results indicate that the ring opening of THFA at the acid site of the Rh-Re catalyst proceeds through the concerted protonation of the oxygen that is to be activated, ring opening and 
hydrogen transfer that proceed via a PCET process and the formation of an oxocarbenium ion intermediate. The presence of both an $\mathrm{OH}$ substituent that resides $\alpha$ to the $\mathrm{C}-\mathrm{O}$ bond that is activated as well as the presence of the protic water solvent act to stabilize the transition state and lower the energy barrier. In aqueous solution, the oxocarbenium ion is not stable and deprotonates to the water solvent to form $\delta$-hydroxyvaleraldehyde. The proton that forms locally in water can then shuttle back to regenerate the surface acid sites. The $\delta$-hydroxyvaleraldehyde that forms can subsequently hydrogenate at sites on the Rh surface via hydrogen addition to the oxygen end of the molecule to form the hydroxylalkyl intermediate which subsequently reacts to give the final 1,5-pentanediol product.

The DFT calculations combined with the experimental observations suggest that the second hydrogenation step of $\delta$-hydroxyvaleraldehyde at the metal site is likely the rate limiting step. The metal sites on the surface are likely covered by water as the reactions are carried out in aqueous media. The rate expression derived from kinetic analysis indicates that the rate of the hydrogenolysis of THFA has a first order dependency on the hydrogen partial pressure and in addition is first order of the concentration of THFA at the low THFA concentration condition, which agrees with the experiments. At significantly higher concentrations of THFA, the THFA hydrogenolysis rates become less dependent on the concentration of THFA, which is also in agreement with experiments.

Ring opening of THFA at different acid sites on Rh-Re surfaces showed that the acid strength of the acid sites as well as the relative coordination number of the neighboring $\mathrm{Rh}$ sites are important in controlling the activation barriers for $\mathrm{C}-\mathrm{O}$ hydrogenolysis. The activation energies for the ring opening of THFA at the terrace, edge and corner Re-OH acid site of the 201atom $\mathrm{Rh}$ cluster are different from one another even though the deprotonation energies of these 
three acid sites are similar. This is due to that the interaction between the hydroxyl group that forms during the ring opening of THFA and $\mathrm{Rh}$ center which is adjacent to the Re-OH acid site. This $\mathrm{Rh}-\mathrm{OH}$ interaction stabilized the transition state and lowers the activation barrier for ring opening. The optimal acid-metal site pair which allows for the highest acidity and greatest $\mathrm{OH}$ stabilization involves the edge Re site and one of the vicinal Rh corner sites.

The ring opening of THFA at the acids sites on Rh-Re surface with different acidities reveal a linear relationship between the adsorption energies for THFA and the resulting activation energies for THFA ring opening. Both are also linearly correlated with the acidity of the Re-OH site as measured by the deprotonation energies. The sites with the highest acidity are those with the lowest activation energies for THFA ring opening. Similar correlations can be used to follow the ring opening of THFA over different bimetal alloys comprised of an reducible metal such as $\mathrm{Rh}, \mathrm{Pt}$, Ir and Pd and an oxophilic promoter metals such as Re, Mo, W and Ru.

The mechanism of the hydrogenolysis of THFA over the Rh-Re catalyst can be extended to other cyclic ethers and polyols such as 1,2-propanediol. The dehydration of 1,2-propanediol at the Rh-Re acid site also proceeds through the concerted protonation, $\mathrm{C}-\mathrm{O}$ bond activation and $\mathrm{H}$ transfer process. The energy barrier for the dehydration of 1,2-propanediol at the more substituted center carbon is about $13 \mathrm{~kJ} / \mathrm{mol}$ lower than that at the less substituted terminal carbon, leads to higher selectivity towards 1-propanol, which is in agreement with the experiments. The energy barriers for the $\mathrm{C}-\mathrm{O}$ bond activation of various cyclic ethers and polyols at the Rh-Re acid site can be linearly correlated to their gas phase carbenium ion formation energies leading to the possibility of predicting the reactivity of different oxygenates over the Rh-Re catalyst by simply calculating the gas phase carbenium ion formation energies. 


\section{Chapter 4-The Nature of the Active Acid Site: Bronsted vs. Lewis Acid}

\subsection{Introduction}

In the previous chapter, we examined the mechanisms of the acid catalyzed $\mathrm{C}-\mathrm{O}$ bond activation of THFA and other cyclic ethers and polyols on the Rh-Re catalyst by analyzing the Brønsted acidity of hydroxyl groups bound to Re atoms within different Rh-Re alloy models. Previous XANES and EXAFS data by Davis et al. ${ }^{[55]}$ suggest that the Re in Pt-Re alloys is reduced under reaction conditions but is never fully reduced to $\operatorname{Re}(0)$. The results suggest that $\operatorname{Re}$ is in an average oxidation state of +2 and is of the form Re-OH. The ideas were subsequently extended to the Rh-Re alloys. The XANES and EXAFS carried out by Tomishige et al. ${ }^{[62]}$ on RhRe catalyst also suggest that $\operatorname{Re}$ is partially reduced and in an average oxidation state of $+1 \sim+2$. Recent XANES and EXAFS data by Miller et al. ${ }^{[73]}$ show nearly identical trends as the data reported by Davis et al. and Tomishige et al.. However, they have different interpretations of the data. They think the Re XANES edge energy and white-line intensity is distorted by using the Re powder as the reference for $\operatorname{Re}(0)$ due to the non-uniform sample pellet preparation and signal re-adsorption by large Re particles. Instead, they focus on the EXAFS spectra and find no evidence of Re-O coordination after the pretreatment of Rh-Re catalyst at the temperature higher than $363 \mathrm{~K}$. Thus, they suggest that the Re is fully reduced and that the active sites are either metallic $\mathrm{Re}$ atoms or adsorbed water $\left(\mathrm{Re}-\mathrm{OH}_{2}\right)$ for the hydrogenolysis of cyclic ethers and polyols.

While the state of the $\mathrm{Re}$ in the $\mathrm{Rh}-\mathrm{Re}$ and Pt-Re catalysts and the active sites that are responsible for the hydrogenolysis of cyclic ethers and polyols are still under debate by experimental groups, herein we use theory to examine and compare three different models of the active acid sites which are consistent with partially oxidized and fully reduced Re sites. They 
include our initial Re-OH Brønsted acid site model, unoccupied Re Lewis acid site or $\mathrm{Re}-\mathrm{OH}_{2}$ Brønsted acid site model where the protons on the adsorbed water act as the active sites as shown in Figure 4.1.

The Brønsted acidity of bound water to metal ions is well-established in the metal aquo ion complexes, ${ }^{[130]}$ and the presence of similar $\mathrm{Re}-\mathrm{OH}_{2}$ species could carry out catalysis in the alloys. In addition, the presence of unoccupied $R e$ centers on the $\mathrm{Rh}-\mathrm{Re}$ surface can also function as Lewis acids as low valence $\operatorname{Re}(\mathrm{I})$ complexes are known Lewis acids. ${ }^{[131-137]}$

Herein we examine the acid properties and ring opening of THFA for the unoccupied Re sites and the adsorbed water $\mathrm{Re}-\mathrm{OH}_{2}$ on the $\mathrm{Rh}-\mathrm{Re}$ alloy surface and compare with our previous results on the acidity and reactivity of the Re-OH sites. We will also examine the adsorption and reactivity of water on the Re centers and analyze the relative amounts of these different sites on the Rh-Re alloy surface at the reaction conditions. This work will help to provide a more complete understand the hydrogenolysis of cyclic ethers and polyols over the Rh-Re catalysts. It will provide insights into other reactions such as the aqueous phase reforming of glycerol, ${ }^{[138-140]}$ water-gas-shift ${ }^{[117,141]}$ and carboxylic acid hydrogenation reactions ${ }^{[142,143]}$ that have been reported over the Pt-Re, Ru-Re and Ir-Re bimetallic catalysts.

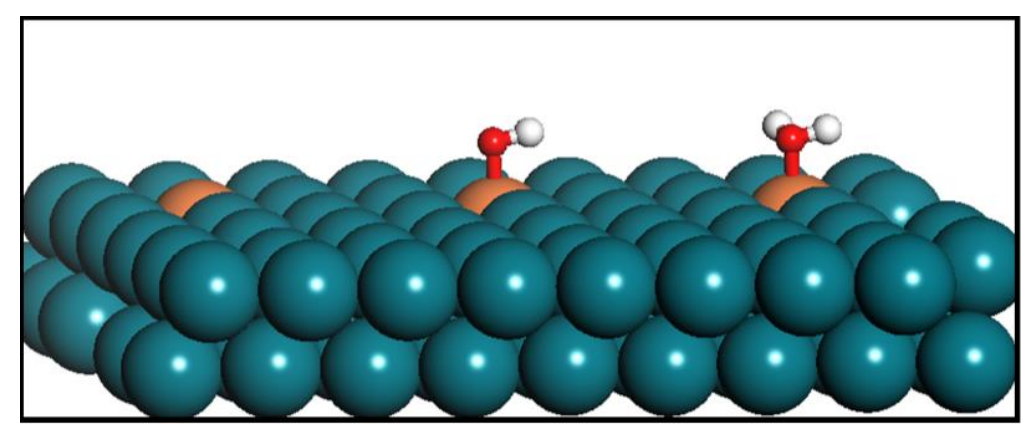

Figure 4.1 Possible acid sites on the Rh-Re alloy surface: the unoccupied Re site, the adsorbed hydroxyl Re-OH and the adsorbed water $\mathrm{Re}-\mathrm{OH}_{2}$. 


\subsection{Computational Methods}

All of the calculations reported herein were carried out using periodic plane-wave gradientcorrected density functional theory methods implemented in the Vienna ab initio Simulation Package (VASP). ${ }^{[89-92]}$ The PW91 ${ }^{[93]}$ form of GGA exchange correlation functional was used to provide the non-local gradient-corrections to exchange and correlation energies. Wave functions were constructed by using projector augmented wave potentials (PAW) ${ }^{[94,95]}$ within a cutoff energy of $396.0 \mathrm{eV}$.

The Rh-Re alloy surface was modeled by using a $\mathrm{Rh}(111)$ surface where well dispersed $\mathrm{Re}$ atom are substituted for $\mathrm{Rh}$ centers to create the $\mathrm{Rh}_{8} \mathrm{Re}_{1} / \mathrm{Rh}(111)$ surface alloy shown in Figure 4.2. The $\mathrm{Rh}(111)$ substrate was modeled using a $3 \times 3$ unit cell with four metal layers and a $15 \AA$ vacuum region above the metal layers, where the top two layers were allowed to relax and the bottom two held fixed to their lattice constant for $\mathrm{Rh}$. The solution phase above the $\mathrm{Rh}$ surface was modeled by filling the vacuum region with 24 water molecules that are randomly placed so that the density of water between the metal surfaces is close to $1 \mathrm{~g} / \mathrm{cm}^{3}$. Ab initio molecular dynamics (NVT ensemble) simulation were carried out at $400 \mathrm{~K}$ for $2.0 \mathrm{ps}$ with the time step of $1.0 \mathrm{fs}$ to equilibrate the water system before the structure optimizations. All of the metal atoms were fixed in the ab initio MD studies carried out here. Nose-Hoover ${ }^{[114,115]}$ thermostat was used to control the temperature during the run. For the structure optimizations, a 6x6x1 MonkhorstPack ${ }^{[96]} \mathrm{k}$-point mesh was used to sample the first Brillouin zone, the electronic energies were converged to within $1 \times 10^{-6} \mathrm{eV}$ and forces on each atom were optimized to within $0.05 \mathrm{eV} / \AA$. For the ab initio molecular dynamics simulations, a $3 \times 3 \times 1$ Monkhorst-Pack ${ }^{[6]}$ k-point mesh was used, and the electronic energies were converged to within $1 \times 10^{-4} \mathrm{eV}$.

The metal particles were modeled using 201-atom cubo-ocoahedral clusters as shown in 
Figure 4.2. Calculations on metal particles were carried non-spin-polarized in a cubic unit cell with a minimum of $12 \AA$ of vacuum separating particles between periodic cells. All structure optimizations were carried out until the maximum force upon any atom was less than $0.05 \mathrm{eV} / \AA$. Electronic energies were converged to within $1 \times 10^{-6} \mathrm{eV}$. These calculations were performed using the $\gamma$-point version of VASP. Monopole and dipole moments of the cell were calculated and used to correct the energy. For charged calculations, quadrupole corrections were also applied.

Transition state calculations for ring-opening of $T H F A$ on the metal surfaces and metal clusters were carried out by first determining the minimum energy path (MEP) using the NEB method ${ }^{[97]}$ using 16 images along the reaction coordinate and converged to $0.25 \mathrm{eV} / \AA$. The results were used to generate initial structures for the dimer method ${ }^{[98]}$ which was then run to the same level of accuracy as the optimizations described above in order to isolate the transition state.

Charge analyses were performed by using the QUAMBO method, ${ }^{[99-102]}$ which transforms the wave-functions of the VASP calculation into spatially localized non-orthogonal quasi-atomic orbitals. This transformation provides information about the atomic orbitals of the system, and permits a Löwdin charge and bond order analysis. ${ }^{[103,104]}$

The deprotonation energy (DPE) of a general Brønsted acid $\mathrm{MOH}\left(\mathrm{MOH}\right.$ can be $\mathrm{Re}-\mathrm{OH}_{2}$ or Re-OH here) was calculated by:

$$
D P E=E\left(M O^{-}\right)+E\left(H^{+}\right)-E(M O H)
$$

where $E\left(\mathrm{MO}^{-}\right)$is the total energy of the conjugate base of the Brønsted acid $\mathrm{MOH}$ after removing a proton, $E\left(H^{+}\right)$is the total energy of the proton calculated in the vacuum. $E(M O H)$ is the total energy of the Brønsted acid $M O H$.

The binding energies of the adsorbates at the acid sites or metal sites were calculated as: 


$$
\Delta E_{a d s}=E_{M+i}-E_{M}-E_{i}
$$

where $E_{M+\mathrm{i}}$ is the total energy of the acid or metal with the adsorbate bound to it, $E_{M}$ is the total energy of the bare acid or metal and $E_{i}$ is the total energy of the adsorbate $i$ in gas phase.

The activation barriers and reaction energies were calculated as:

$$
\begin{gathered}
\Delta E_{A C T}=E_{T S}-E_{R} \\
\Delta E_{r x n}=E_{P}-E_{R}
\end{gathered}
$$

respectively, where $E_{T S}$ is the total energy of the transition state, $E_{R}$ is the total energy of the reactant state and $E_{P}$ is the total energy of the product state.

In order to determine the surface coverages of the $\mathrm{OH}_{\mathrm{x}}{ }^{*}$ intermediates at the Re sites on the Rh-Re surface under reaction conditions we examined the overall free energies of reaction. The free energies for each state were calculated as:

$$
G=E+Z P V E+G_{v i b}+G_{\text {trans }}+G_{\text {rot }}
$$

where $E$ is the DFT calculated total energy, $Z P V E$ is the zero point energy. $G_{v i b}, G_{t r a n s}, G_{r o t}$ are the vibrational, translational and rotational free energy. For calculations of adsorbed species $\left(\mathrm{H}_{2} \mathrm{O}^{*}, \mathrm{OH}^{*}\right.$ and $\left.\mathrm{O}^{*}\right)$ on the metal surface, we assume that there are no translational or rotational degrees of freedom and as such set $G_{\text {trans }}$ and $G_{r o t}$ to zero. The $Z P V E$ and $G_{v i b}$ were calculated by vibrational frequencies derived from DFT frequency calculations in which the electronic energies were converged to within $1 \times 10^{-6} \mathrm{eV}$. The translational and rotational degrees of freedom and $G_{\text {trans }}$ and $G_{\text {rot }}$ for $\mathrm{H}_{2}$ and $\mathrm{H}_{2} \mathrm{O}$ were calculated by statistical thermodynamics. 

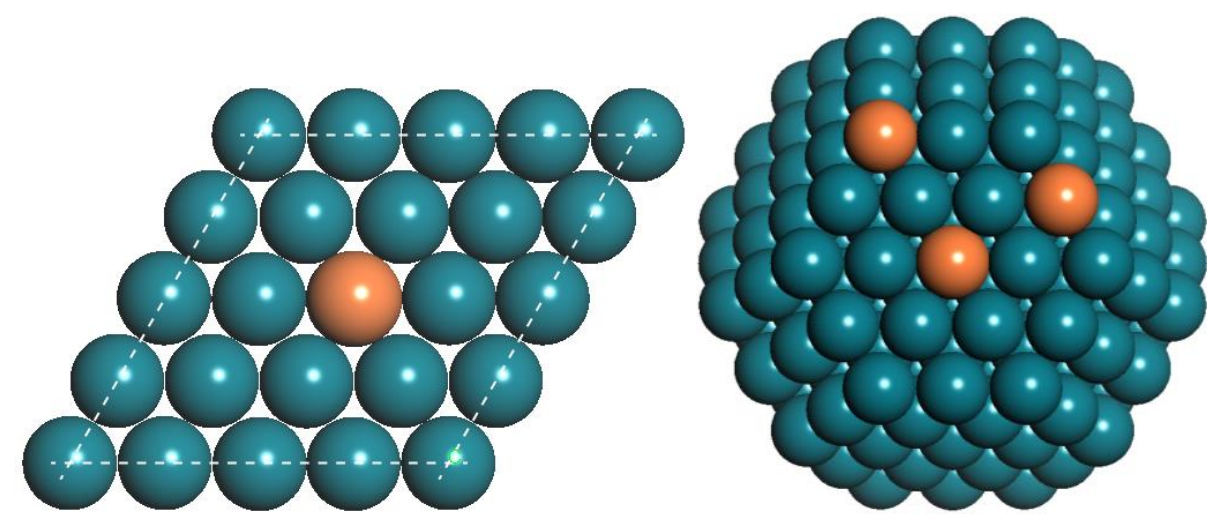

Figure 4.2 The $4 \times 4 \mathrm{Rh}(111)$ surface with well dispersed Re site (left) and the 201-atom cubooctahedral Rh particle with Re located at different sites (right) used to model Rh-Re catalyst. The adsorbed species such as $\mathrm{H}_{2} \mathrm{O}$ and $\mathrm{OH}$ at the Re sites are not shown.

\subsection{Results and Discussions}

\subsubsection{Acid properties of $\mathrm{Re}-\mathrm{OH}_{2}$ and unoccupied Re site on the Rh-Re alloy surface}

The deprotonation energy $(D P E)$ of the surface hydroxyl provides a direct probe of the Brønsted acidity of a solid acid. We calculated the $D P E$ of the adsorbed water $\left(\mathrm{Re}-\mathrm{OH}_{2}\right)$ as well as adsorbed hydroxyl intermediates $(\mathrm{Re}-\mathrm{OH})$ bound to the Re sites on the Rh-Re alloy surface where the Re atoms were placed at the terrace, edge and corner site of the Rh particle in order to examine the effects of the coordinative unsaturated metal on its acidity. The $D P E$ values for water adsorbed to Re located at the (111) terrace site $\left(\mathrm{Re}-\mathrm{OH}_{2}\right)$ was calculated to be $1174 \mathrm{~kJ} / \mathrm{mol}$, which is about $19 \mathrm{~kJ} / \mathrm{mol}$ higher and somewhat less acidic than the $D P E$ of the adsorbed $\mathrm{OH}$ at the same Re terrace site on the Rh-Re surface $(1145 \mathrm{~kJ} / \mathrm{mol})$. While the $D P E$ for the adsorbed water is somewhat less acidic than the $\mathrm{OH}^{*}$, it is within the range of $1050-1200 \mathrm{~kJ} / \mathrm{mol}$ for other well-known solid acids including heteropolyacids (HPAs) ${ }^{[144]}$ and zeolites ${ }^{[118]}$ indicating the adsorbed water $\mathrm{Re}-\mathrm{OH}_{2}$ can act as a Brønsted acid. The DPE for water bound to Re at the edge of the Rh particle (edge $\mathrm{Re}-\mathrm{OH}_{2}$ ) was calculated to be $1116 \mathrm{~kJ} / \mathrm{mol}$ and thus more acidic than that at the terrace site. The acidity for water bound to the corner Re site was found the strongest 
resulting in a $D P E$ of $1076 \mathrm{~kJ} / \mathrm{mol}$. It is noted that the $D P E$ values for water bound to Re at the corner and edge sites are actually lower than those for adsorbed $\mathrm{OH}$ at these same sites as shown in Table 4.1, thus indicating that $\mathrm{Re}-\mathrm{OH}_{2}$ is actually more acidic than $\mathrm{Re}-\mathrm{OH}$ at these sites.

Table 4.1 DFT calculated DPE of the Brønsted acid $\mathrm{Re}-\mathrm{OH}_{2}, \mathrm{Re}-\mathrm{OH}$ sites on the Rh-Re particle, ammonia adsorption energies at the $\mathrm{Re}-\mathrm{OH}_{2}, \mathrm{Re}-\mathrm{OH}$ sites and the unoccupied Re sites, and the adsorption energies of $\mathrm{H}_{2} \mathrm{O}, \mathrm{OH}$ and $\mathrm{O}$ at the Re sites on the $\mathrm{Rh}-\mathrm{Re}$ particle. The units are in $\mathrm{kJ} / \mathrm{mol}$.

\begin{tabular}{llll}
\hline & Terrace & Edge & Corner \\
\hline$D P E\left(\mathrm{Re}-\mathrm{OH}_{2}\right)$ & 1174 & 1116 & 1076 \\
$D P E(\mathrm{Re}-\mathrm{OH})$ & 1145 & 1146 & 1145 \\
\hline $\mathrm{NH}_{3}-E_{a d s}\left(\mathrm{Re}-\mathrm{OH}_{2}\right)$ & -60 & -95 & -107 \\
$\mathrm{NH}_{3}-E_{a d s}(\mathrm{Re}-\mathrm{OH})$ & -70 & -69 & -70 \\
$\mathrm{NH}_{3}-E_{a d s}(\mathrm{Re})$ & -95 & -114 & -141 \\
\hline$\Delta E_{a d s}\left(\mathrm{H}_{2} \mathrm{O}\right)$ at $\mathrm{Re}$ & -43 & -55 & -80 \\
$\Delta E_{a d s}(\mathrm{OH})$ at $\mathrm{Re}$ & -314 & -385 & -450 \\
$\Delta E_{a d s}(\mathrm{O})$ at $\mathrm{Re}$ & -549 & -617 & -682 \\
$\Delta E_{a d s}(\mathrm{OH})-\Delta E_{a d s}\left(\mathrm{H}_{2} \mathrm{O}\right)$ & -270 & -330 & -370 \\
$\Delta E_{a d s}(\mathrm{O})-\Delta E_{a d s}(\mathrm{OH})$ & -235 & -232 & -232
\end{tabular}

The adsorption energy for a base molecule such as ammonia is often used experimentally to evaluate the solid acidity for both Brønsted as well as Lewis acids. The methods, however, often measure (experimentally) other properties and as such cannot be used to faithfully examine solid acidity alone. We can often omit these other interactions to create theoretical probes of the ideal acid site strength. As shown in Table 4.1, the ammonia adsorbs at the terrace, edge and corner $\mathrm{Re}-\mathrm{OH}_{2}$ site with the adsorption energy of $-60 \mathrm{~kJ} / \mathrm{mol},-95 \mathrm{~kJ} / \mathrm{mol}$ and $-107 \mathrm{~kJ} / \mathrm{mol}$ respectively. 
This trend for the ammonia adsorption energy at the terrace, edge and corner $\mathrm{Re}-\mathrm{OH}_{2}$ site is consistent with the DPE calculations shown above.

Comparing the deprotonation energies of the adsorbed water $\mathrm{Re}-\mathrm{OH}_{2}$ with the adsorbed hydroxyl Re-OH, we can see that the DPEs of $\mathrm{Re}-\mathrm{OH}_{2}$ at the terrace, edge and corner sites are markedly different while the DPEs of Re-OH at these three sites are almost the same. We have shown previously ${ }^{[129]}$ that the DPE of the adsorbed $\mathrm{OH}$ at different bimetallic alloy sites can be decomposed to the dehydrogenation energy (DHE) of the adsorbed $\mathrm{OH}$ and the electron affinity $(E A)$ of the metal particles, where the dehydrogenation energy can further be correlated to the difference of the binding energy of $\mathrm{O}$ and the binding energy of $\mathrm{OH}$ at the alloy site $\left[\Delta E_{\text {ads }}(\mathrm{O})-\right.$ $\left.\triangle E_{\text {ads }}(\mathrm{OH})\right]$. Similarly we found that the $D P E$ of the adsorbed $\mathrm{H}_{2} \mathrm{O}$ at the different Re sites on the $\mathrm{Rh}-\mathrm{Re}$ surface can be correlated to the dehydrogenation energy of the adsorbed $\mathrm{H}_{2} \mathrm{O}$ and as a result to the difference between the binding energy of $\mathrm{OH}$ and that of $\mathrm{H}_{2} \mathrm{O}$ at the Re sites $\left[\Delta E_{a d s}\right.$ $\left.(\mathrm{OH})-\Delta E_{a d s}\left(\mathrm{H}_{2} \mathrm{O}\right)\right]$. As shown in Table 4.1, $\mathrm{H}_{2} \mathrm{O}, \mathrm{OH}$ and $\mathrm{O}$ all show a decrease in the binding energy in moving from:

Re corner site (strongest) $>$ Re edge site $>$ Re 111 terrace site (weakest).

The changes for the binding energy of $\mathrm{H}_{2} \mathrm{O}^{*}$ which is a stable and saturated molecule at these three different sites are not as significant as the changes that result for the unsaturated $\mathrm{OH}^{*}$ and $\mathrm{O} *$ intermediates. This results in different values of $\left[\Delta E_{a d s}(\mathrm{OH})-\Delta E_{a d s}\left(\mathrm{H}_{2} \mathrm{O}\right)\right]$ and thus different deprotonation energies at the terrace, edge and corner $\mathrm{Re}-\mathrm{OH}_{2}$ sites.

In the case of $\mathrm{OH}^{*}$ adsorption, the changes of the binding energy of $\mathrm{OH}^{*}$ at the terrace, edge and corner sites are offset by the changes of the binding energy of $\mathrm{O}^{*}$, which results in values for $\left[\Delta E_{a d s}(\mathrm{O})-\Delta E_{a d s}(\mathrm{OH})\right]$ that are similar at each site and thus deprotonation energies that are similar. In the case for water adsorption, there is a much greater dependence of $D P E$ on 
the $\left[\Delta E_{a d s}(\mathrm{OH})-\Delta E_{a d s}\left(\mathrm{H}_{2} \mathrm{O}\right)\right]$ values as the changes in $\Delta E_{a d s}\left(\mathrm{H}_{2} \mathrm{O}\right)$ do not offset the changes in $\Delta E_{a d s}(\mathrm{OH})$. As a result there is strong linear relationship between $D P E$ and the values of $\left[\Delta E_{a d s}\right.$ $\left.(\mathrm{OH})-\Delta E_{a d s}\left(\mathrm{H}_{2} \mathrm{O}\right)\right]$ as is shown in Figure 4.3.

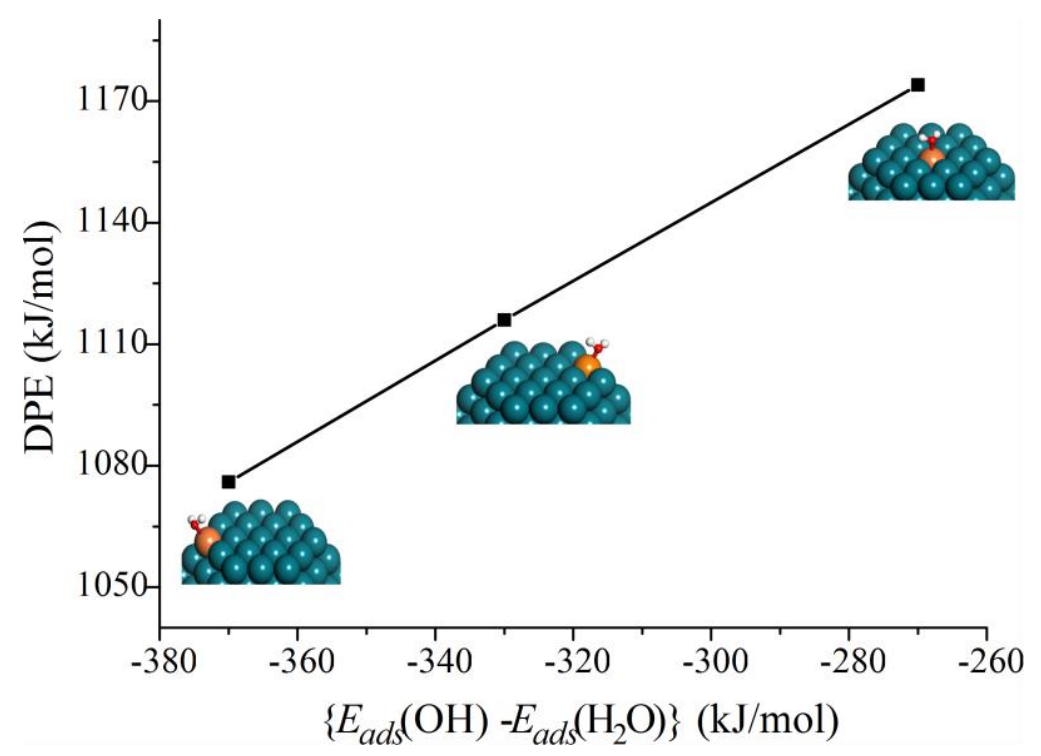

Figure 4.3 Linear correlation of the deprotonation energies of $\mathrm{Re}-\mathrm{OH}_{2}$ with $\mathrm{Re}$ atom located at the terrace, edge and corner site of the $\mathrm{Rh}-\mathrm{Re}$ particle versus the difference of the binding energies of $\mathrm{OH}$ and $\mathrm{H}_{2} \mathrm{O}$ at the Re atoms $\left[\Delta E_{a d s}(\mathrm{OH})-\Delta E_{a d s}\left(\mathrm{H}_{2} \mathrm{O}\right)\right]$.

We subsequently examined the adsorption of ammonia which acts as a base and can be used to further examine the acid properties of the $\mathrm{Re}-\mathrm{OH}_{2}, \mathrm{Re}-\mathrm{OH}$ and Re sites. The results for the ammonia adsorption studies reported in Table 4.1, indicate that ammonia strongly binds to the Brønsted acid sites for $\mathrm{OH}$ and $\mathrm{H}_{2} \mathrm{O}$ on Re as well as the Lewis acid Re sites. The adsorption of ammonia to Re sites that reside at the terrace, edge and corner sites were calculate to be -95 $\mathrm{kJ} / \mathrm{mol},-114 \mathrm{~kJ} / \mathrm{mol}$ and $-141 \mathrm{~kJ} / \mathrm{mol}$, respectively. Similar trends were also found for the adsorption of $\mathrm{H}_{2} \mathrm{O}, \mathrm{OH}$ and $\mathrm{O}$ at these same three sites. The strong oxophilicity of $\mathrm{Re}$ and the low occupancy of the d-states on Re, together with the strong binding of ammonia to the Re sites within the Rh-Re alloy may suggest that Re acts as a Lewis acid. The Brønsted acidity of the 
$\mathrm{H}_{2} \mathrm{O}$ and $\mathrm{OH}$ that bind at the Re atoms on the Rh-Re alloy surface also suggest that vacant $\mathrm{Re}$ sites will act as a Lewis acid. The deprotonation of $\mathrm{H}_{2} \mathrm{O}$ or $\mathrm{OH}$ intermediates that bind to Re sites result in the formation of a proton and a negative charged conjugate base which is characteristic of a Lewis acid catalyzed reaction where the negative charged center at the Lewis acid site and a positively charged carbenium ion site result in the reported Lewis acid acidity. If the Re atoms on the $\mathrm{Rh}$-Re alloy surface facilitate the deprotonation of $\mathrm{H}_{2} \mathrm{O}$ or $\mathrm{OH}$, they should also facilitate the Lewis acid catalyzed C-O bond activation of cyclic ethers and polyols.

\subsubsection{Charge analysis of the unoccupied Re site, $\mathrm{Re}-\mathrm{OH}$ and $\mathrm{Re}-\mathrm{OH}_{2}$ sites}

Detailed charge analyses of the unoccupied $\mathrm{Re}, \mathrm{Re}-\mathrm{OH}$ and the $\mathrm{Re}-\mathrm{OH}_{2}$ sites on the $\mathrm{Rh}-\mathrm{Re}$ particle surface were carried out to probe the nature of the active Re sites. The results are shown in Table 4.2. Previous results reported by Miller et al. ${ }^{[73]}$ indicate that Re in the active Rh-Re catalysts is fully reduced whereas results from Tomishige et al. ${ }^{[62]}$ for Rh-Re and Davis et al. ${ }^{[85]}$ for Pt-Re indicate that the Re is partially charged. These differences have led to different speculations as to the nature of the active site and its charge state under reaction conditions.

Table 4.2 Charge analyses of the unoccupied Re atoms, the adsorbed water $\mathrm{Re}-\mathrm{OH}_{2}$ and hydroxyl $\mathrm{Re}-\mathrm{OH}$ with Re located at the terrace, edge and corner sites of the Rh-Re particle*

\begin{tabular}{lcccccc}
\hline & \multicolumn{2}{c}{ Terrace } & \multicolumn{2}{c}{ Edge } & \multicolumn{2}{c}{ Corner } \\
\hline & $\mathrm{Re}$ & $\mathrm{Rh}_{200}$ & $\mathrm{Re}$ & $\mathrm{Rh}_{200}$ & $\mathrm{Re}$ & $\mathrm{Rh}_{200}$ \\
\hline Unoccupied Re & +0.39 & -0.39 & +0.34 & -0.34 & +0.29 & -0.29 \\
$\mathrm{Re}-\mathrm{OH}$ & +0.64 & -0.35 & +0.62 & -0.34 & +0.60 & -0.33 \\
$\mathrm{Re}-\mathrm{OH}_{2}$ & +0.49 & -0.62 & +0.43 & -0.58 & +0.38 & -057 \\
& & & & & & \\
\hline
\end{tabular}

* The charges on the adsorbates $\mathrm{H}_{2} \mathrm{O}$ or $\mathrm{OH}$ are not listed, as they can be calculated by taking the negative of the sum of the charges on the $\mathrm{Rh}$ and $\mathrm{Re}$ atoms. 
The results in Table 4.2 clearly indicate that Re is positively charged for all three of the active site models and that the charge on the Re increases from:

Lewis acid $\mathrm{Re}(+0.39)<$ Brønsted acid Re- $\mathrm{OH}_{2}(+0.49)<$ Brønsted acid Re-OH $(+0.64)$

We see that even in the metallic $\mathrm{Rh}-\mathrm{Re}$ alloy, the bare Re site is partially positive charged at +0.39 for $\mathrm{Re}$ embedded within the $\mathrm{Rh}$ terraces. The local $\mathrm{Rh}$ atoms become more negatively charged as there is a direct charge transfer. The coordination number of the Re atom which directly reflects the number of bonds to neighboring $\mathrm{Rh}$ atoms influences charge transfer. The charge on Re decreases by 0.1 in moving from the terrace site with $9 \mathrm{Rh}$ neighbors $(+0.39)$ to a corner site with $7 \mathrm{Rh}$ neighbors $(+0.29)$.

The positive charge at the Re site, increases upon the adsorption of water. Water donates electrons to the metal particle thus leading to a more negative charge on the overall $\mathrm{Rh}-\mathrm{Re}$ particle. The charge on the Re atom to which water is adsorbed, however is more positively charged $\left(+0.38\right.$ for the corner $\mathrm{Re}-\mathrm{OH}_{2}$ site and +0.49 for the terrace $\mathrm{Re}-\mathrm{OH}_{2}$ site) as shown in Table 4.2. The charge on the Re increase further upon the adsorption of $\mathrm{OH}$ as $\mathrm{OH}$ withdraws electrons from the metal particle thus resulting in a more positive charge on the $\mathrm{Re}$ in the $\mathrm{Rh}-\mathrm{Re}$ particle $(+0.60 \sim+0.64)$.

XANES characterization results for the Rh-Re catalyst by Tomishige et al ${ }^{[62]}$ indicate that the $\operatorname{Re}$ is positive charged with the oxidation state of about $+1 \sim+2$. Our charge analyses show that all the three forms of Re atom (unoccupied Re, the adsorbed hydroxyl Re-OH and the adsorbed water $\mathrm{Re}-\mathrm{OH}_{2}$ ) are all positively charged to varying degrees with $\mathrm{Re}-\mathrm{OH}$ being the largest. It is noted that the charge that is calculated is a partial charge. It is difficult to formally assign shared electron density to specific atoms. As a result the calculated charges are typically less than the formal charge established from experiments. In addition, the experiments measure 
the mixed oxidation states of the Re atoms and cannot distinguish between the different Re atoms on the $\mathrm{Rh}-\mathrm{Re}$ catalysts. The partial positive charge on the metallic $\mathrm{Re}$ atoms on the $\mathrm{Rh}-\mathrm{Re}$ alloy surface would suggest that Re atoms in the Rh-Re surface allow is Lewis acidic similar to many low valence $\operatorname{Re}(\mathrm{I})$ complexes reported experimentally ${ }^{[131-137]}$ and adsorb or activate water to form Brønsted acid sites.

\subsubsection{Ring Opening of THFA at the unoccupied Re site and the $\mathrm{Re}-\mathrm{OH}_{2}$ site}

The reaction path and the corresponding reaction energies for the acid-catalyzed ring opening of the cyclic ether THFA at the $\mathrm{Re}-\mathrm{OH}$ acid sites examined in Chapter 3 indicate that THFA ring opens at the $\mathrm{Re}-\mathrm{OH}$ acid sites via a concerted protonation, ring opening and $\mathrm{H}-$ transfer mechanism to form an oxocarbenium ion. We extend these studies here to examine the ring opening of THFA at the Brønsted acid $\mathrm{Re}-\mathrm{OH}_{2}$ sites and the Lewis acid unoccupied Re sites on a $\mathrm{Rh}$ (111) surface model with well dispersed Re in the surface. As shown in Figure 4.4, THFA binds at the $\mathrm{Re}-\mathrm{OH}_{2}$ site through the ether oxygen with the binding energy of $-43 \mathrm{~kJ} / \mathrm{mol}$, which is about $10 \mathrm{~kJ} / \mathrm{mol}$ weaker than at the $\mathrm{Re}-\mathrm{OH}$ site $(-53 \mathrm{~kJ} / \mathrm{mol})$. The transition state for the ring opening of THFA at the $\mathrm{Re}-\mathrm{OH}_{2}$ site is similar to that at the $\mathrm{Re}-\mathrm{OH}$ site as shown in Figure 4.4. The presence of water from the aqueous media can help to stabilize the transition state. The energy barrier calculated with additional water was calculated to be $96 \mathrm{~kJ} / \mathrm{mol}$, which is about 12 $\mathrm{kJ} / \mathrm{mol}$ higher than at the Re-OH site. The weaker binding of THFA and the higher energy barrier for its ring opening at the $\mathrm{Re}-\mathrm{OH}_{2}$ site than at the $\mathrm{Re}-\mathrm{OH}$ acid site is due to the weaker acidity of the terrace $\mathrm{Re}-\mathrm{OH}_{2}$ than the terrace $\mathrm{Re}-\mathrm{OH}$ as shown above. 


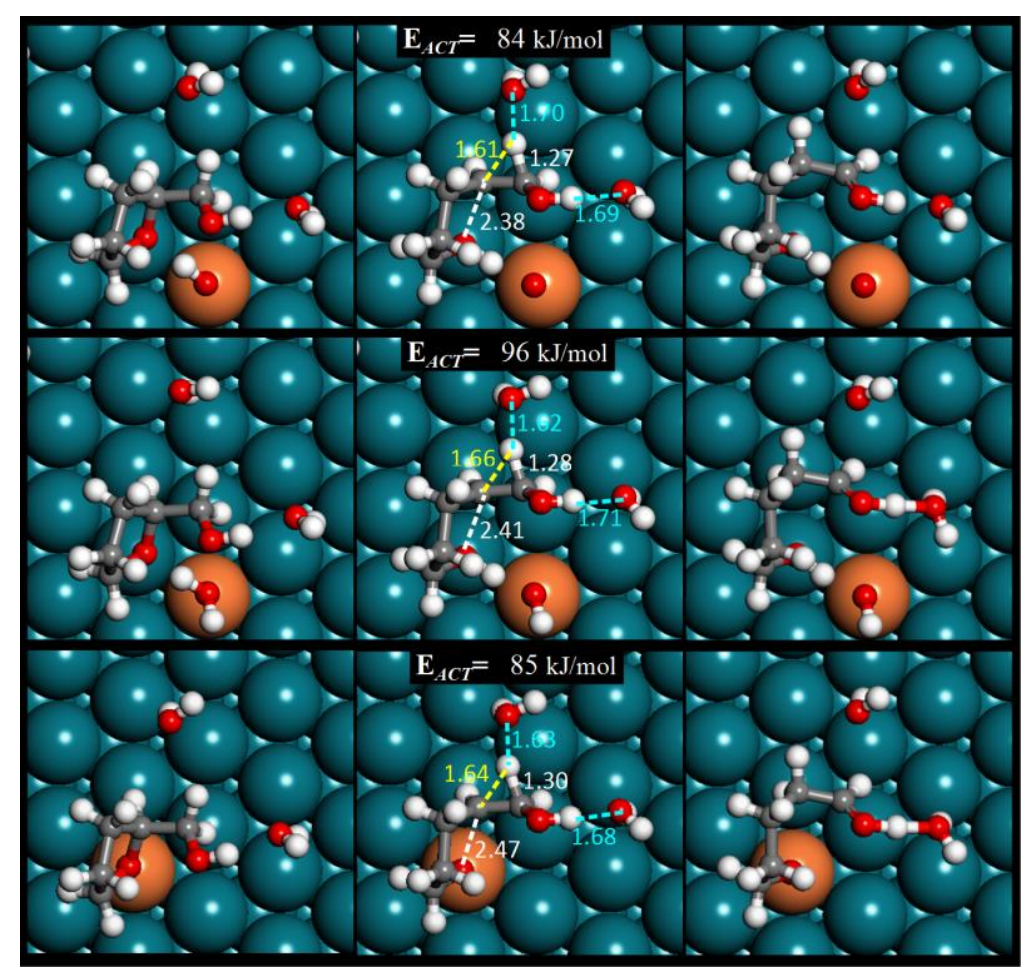

Figure 4.4 DFT calculated reactant, transition state and product structures for the ring opening of THFA at the Re-OH, Re- $\mathrm{OH}_{2}$ and unoccupied Re sites on the Rh-Re surface.

The ring opening of THFA at the $\mathrm{Re}-\mathrm{OH}_{2}$ sites were subsequently carried out on the more representative 201-atom cubo-octahedral cluster model as shown in Figure 4.5 with Re atom locating at the terrace, edge and corner of the particle. As shown in Table 4.3, the binding energy of THFA at the terrace $\mathrm{Re}-\mathrm{OH}_{2}$ site is calculated to be $-42 \mathrm{~kJ} / \mathrm{mol}$, which is similar to the surface model $\mathrm{Re}-\mathrm{OH}_{2}$ site reported above. The energy barrier is also similar $(98 \mathrm{~kJ} / \mathrm{mol})$, indicating the simple surface modelled $\mathrm{Re}-\mathrm{OH}_{2}$ provides a good representation of the (111) terrace sites on $\mathrm{Rh}$ metal particles. THFA was calculated to bind more strongly to the uncoordinatively saturated edge and corner $\mathrm{Re}-\mathrm{OH}_{2}$ sites with adsorption energies of $-65 \mathrm{~kJ} / \mathrm{mol}$ and $-85 \mathrm{~kJ} / \mathrm{mol}$ respectively, which is consistent with the increasing acidity of $\mathrm{Re}-\mathrm{OH}_{2}$ at the terrace $<$ edge $<$ corner site. 
Table 4.3 Adsorption energies of THFA and activation barriers for its ring opening at $\mathrm{Re}-\mathrm{OH}$, $\mathrm{Re}-\mathrm{OH}_{2}$ and unoccupied Re sites. The units are in $\mathrm{kJ} / \mathrm{mol}$.

\begin{tabular}{lllll}
\hline & & Terrace & Edge & Corner \\
\hline \multirow{2}{*}{ Re-OH } & $\Delta E_{\text {ads }}$ & -53 & -54 & -55 \\
& $\Delta E_{A C T}$ & 88 & 38 & 56 \\
& $\Delta E_{A C T}{ }^{r e f^{*}}$ & 76 & 25 & 43 \\
Re-OH & $\Delta E_{\text {ads }}$ & -42 & -65 & -85 \\
& $\Delta E_{A C T}$ & 98 & 17 & 28 \\
& $\Delta E_{A C T}{ }^{r e f^{*}}$ & 84 & -2 & 7 \\
Unoccupied Re & $\Delta E_{a d s}$ & -57 & -80 & -112 \\
& $\Delta E_{A C T}$ & 104 & 60 & 25 \\
& $\Delta E_{A C T}{ }^{r e f^{*}}$ & 90 & 35 & -7
\end{tabular}

* The $\Delta E_{A C T}{ }^{\text {ref }}$ is the energy barrier in reference to the state: THFA ${ }^{\text {gas }}+$ Acid $\cdots \mathrm{H}_{2} \mathrm{O}$.

The activation barriers for the ring opening of THFA at the edge and corner Re- $\mathrm{OH}_{2}$ sites were calculated to be $17 \mathrm{~kJ} / \mathrm{mol}$ and $28 \mathrm{~kJ} / \mathrm{mol}$ respectively, which are much lower than that calculated at the terrace $\mathrm{Re}-\mathrm{OH}_{2}$ site $(98 \mathrm{~kJ} / \mathrm{mol})$. The much lower energy barriers are attributed to the stronger acidity at the edge and corner sites as well of the stronger binding of the hydroxyl group in the transition states to the vicinal coordinatively unsaturated $\mathrm{Rh}$ sites as was discussed for the ring opening of THFA at the different Re-OH sites in Chapter 3. From Figure 4.5, we can see that in the transition state for the ring opening of THFA at the edge $\mathrm{Re}-\mathrm{OH}_{2}$ acid site, the hydroxyl group binds at a corner Rh atom, which is stronger than at a terrace $\mathrm{Rh}$ atom or an edge $\mathrm{Rh}$ atom that is adjacent to the terrace or corner $\mathrm{Re}-\mathrm{OH}_{2}$ acid sites. This neighboring metal site effects is stronger than the effect of acidity at these sites. As a result, the activation energy for the ring opening of THFA at the edge $\mathrm{Re}-\mathrm{OH}_{2}$ acid site is lower than that at the corner $\mathrm{Re}-\mathrm{OH}_{2}$ acid 
site, even though the acidity of $\mathrm{Re}-\mathrm{OH}_{2}$ at the edge site is weaker than that at the corner.

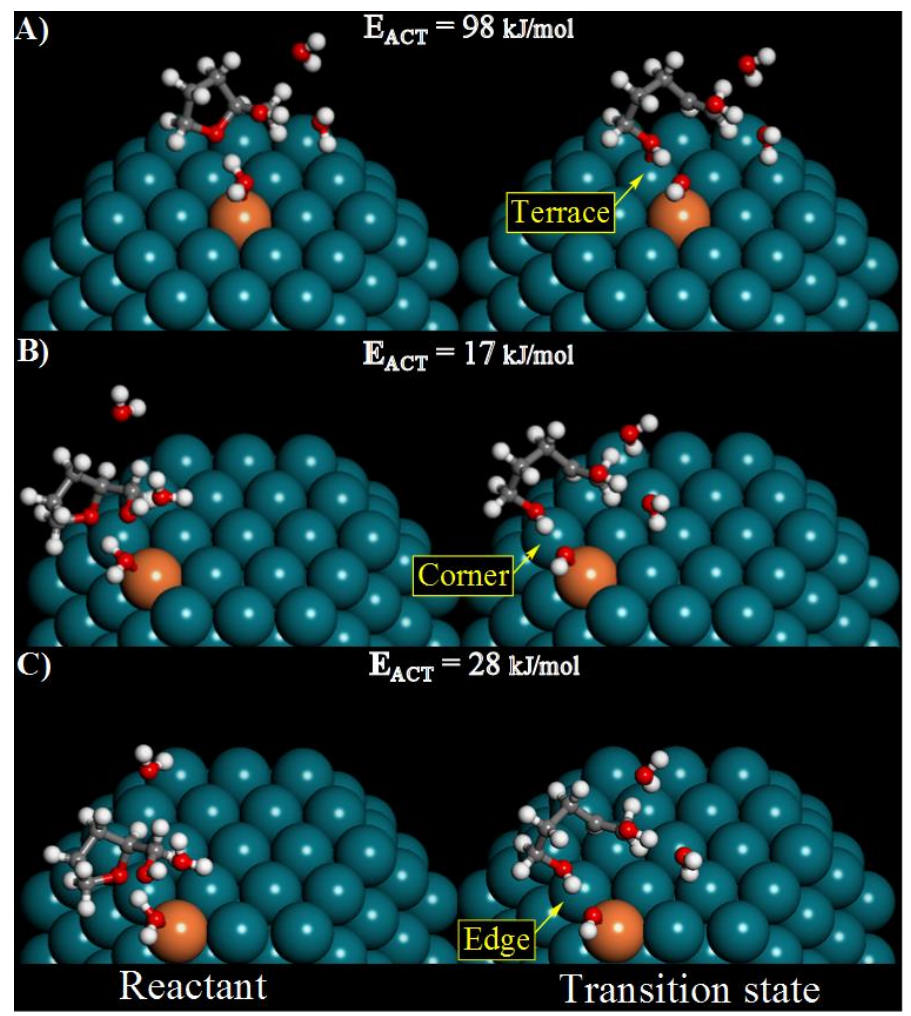

Figure 4.5 DFT calculated reactant and transition state structures for the ring opening of THFA at the terrace, edge and corner $\mathrm{Re}-\mathrm{OH}_{2}$ sites on the $\mathrm{Rh}-\mathrm{Re}$ particle.

The ring opening of THFA at the unoccupied Re atoms were also investigated as they are Lewis acid in character. As shown in Fig 4.4, THFA adsorbs at the Re site in the Rh(111) surface through the ether oxygen with an adsorption energy of $-68 \mathrm{~kJ} / \mathrm{mol}$, which is about $-24 \mathrm{~kJ} / \mathrm{mol}$ stronger than the adsorption of THFA at the pure Rh (111) surface $(-44 \mathrm{~kJ} / \mathrm{mol})$ that we reported previously. ${ }^{\left[{ }^{84}\right]}$ The $\mathrm{Re}^{\delta+}$ Lewis acid site plays that same role as the $\mathrm{H}^{+}$of the Brønsted acid site in that it adds to the oxygen on THFA and promotes the scission of the $\mathrm{C}-\mathrm{O}$ bond. The ring opening reaction proceeds in a concerted manner involving the heterolytic scission of the C-O bond together with a simultaneous $\mathrm{H}$-transfer from $\mathrm{C} 1$ to $\mathrm{C} 2$ to form the oxocarbenium ion, as 
was the case in the ring opening at the Brønsted acid $\mathrm{Re}-\mathrm{OH}$ and $\mathrm{Re}-\mathrm{OH}_{2}$ sites shown in Figure 4.4. The activation barrier for ring opening at the Lewis acid Re site was calculated to be 85 $\mathrm{kJ} / \mathrm{mol}$, which is very similar to that calculated for the ring opening of THFA at the Re-OH Brønsted acid site $(84 \mathrm{~kJ} / \mathrm{mol})$.

A charge analysis showed THFA donates electrons to the Rh-Re metal surface upon adsorption as shown in Figure 4.6 leading to negative charge on the Rh-Re metal (-0.43) and positive charge on the THFA adsorbate $(+0.43)$. Similar to the adsorption of water as discussed above, the charge on the Re atom to which THFA is adsorbed, however is more positively charged (from +0.37 on bare Re to +0.49 on Re-THFA). The scission of the $\mathrm{C}-\mathrm{O}$ proceeds in a heterolytic manner and as such there is a transfer of negative charge into the metal. The negative charge on the Rh-Re alloy increases from -0.43 in the reactant to -0.65 in the transition state as shown in Figure 4.6. This results in an increase in the positive charge on the THFA in the reactant state $(+0.43)$ to $(+0.65)$ in the transition state. This heterolytic charge distribution is very similar to that reported Lewis acid catalyzed ring opening of epoxides. ${ }^{[145]}$

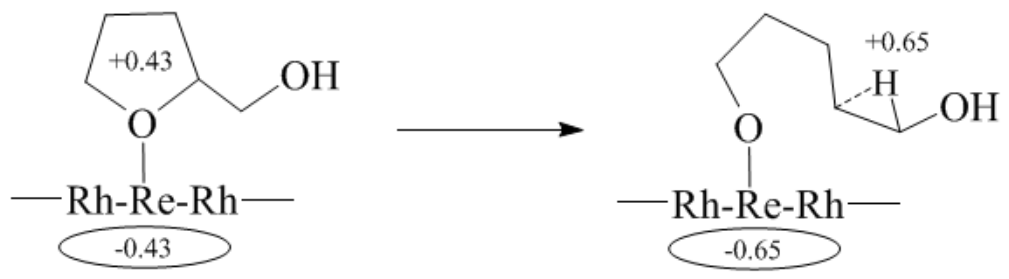

Figure 4.6 Charge analyses of the reactant and transition state structures for the ring opening of THFA at the Re site on the Rh-Re alloy surface.

The ring opening of THFA at the Re site on the Rh-Re alloy surface were also carried out on the more representative 201-atom particle models as shown in Figure 4.7 with Re atom locating at the terrace, edge and corner sites of the particle. The binding energy of THFA at the terrace Re 
site was calculated to be $-57 \mathrm{~kJ} / \mathrm{mol}$, which is $\sim 11 \mathrm{~kJ} / \mathrm{mol}$ weaker than at the Re sites within the $\mathrm{Rh}$ (111) surface model reported above. The activation barrier for the ring opening of THFA at the terrace Re site was calculated to be $104 \mathrm{~kJ} / \mathrm{mol}$, which is also about $19 \mathrm{~kJ} / \mathrm{mol}$ higher than at the Re sites within the $\mathrm{Rh}(111)$ surface reported above. The results here suggest the simple model for the Lewis acid Re sites within the $\mathrm{Rh}(111)$ surface is not a very accurate model for the Re sites within the (111) surface of the 201 atom metal particle. This is different than the results reported above for the $\mathrm{Re}-\mathrm{OH}$ and $\mathrm{Re}-\mathrm{OH}_{2}$ cases where the simple (111) surface was found to be a very good model for the 201 metal particles.

THFA was calculated to bind to the coordinatively less edge and corner Re sites in the RhRe 201 atom cluster much stronger than the terrace Re site with the adsorption energy of $-80 \mathrm{~kJ} / \mathrm{mol}$ and $-112 \mathrm{~kJ} / \mathrm{mol}$ respectively. The activation energies for the ring opening of THFA at the edge and corner Re sites were calculated to be $60 \mathrm{~kJ} / \mathrm{mol}$ and $25 \mathrm{~kJ} / \mathrm{mol}$ respectively, which are much lower than that at the terrace $\mathrm{Re}$ site $(104 \mathrm{~kJ} / \mathrm{mol})$. Earlier we showed the direct correlation between the acidity as measured by the deprotonation energy and the calculated barriers for different Brønsted acid sites. In order to establish the relationship between acidity and activity for the different Lewis acid sites we use ammonia adsorption as a measure of the Lewis acid. As shown in Figure 4.8, the adsorption energy of THFA and the energy barriers for its ring opening at the terrace, edge and corner Re sites can be linearly correlated to the adsorption energy of $\mathrm{NH}_{3}$ at these three sites. As a result, the activation barriers for the ring opening of THFA at these three sites can also be linearly correlated to the adsorption energy of THFA at these three sites, thus direct linear relationship between the acidity of the Re Lewis acid site and its acid catalyzed activity. 


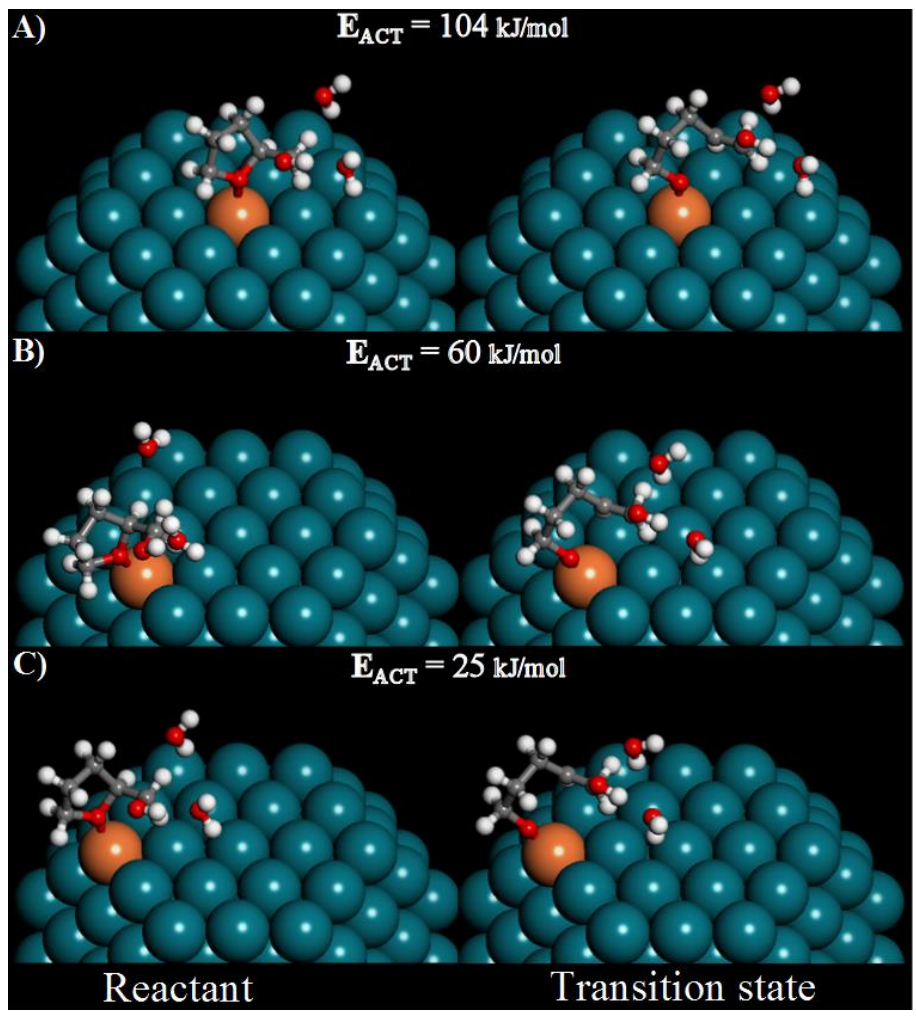

Figure 4.7 DFT calculated reactant and transition state structures for the ring opening of THFA at the terrace, edge and corner Lewis acid Re sites on the Rh-Re particle.

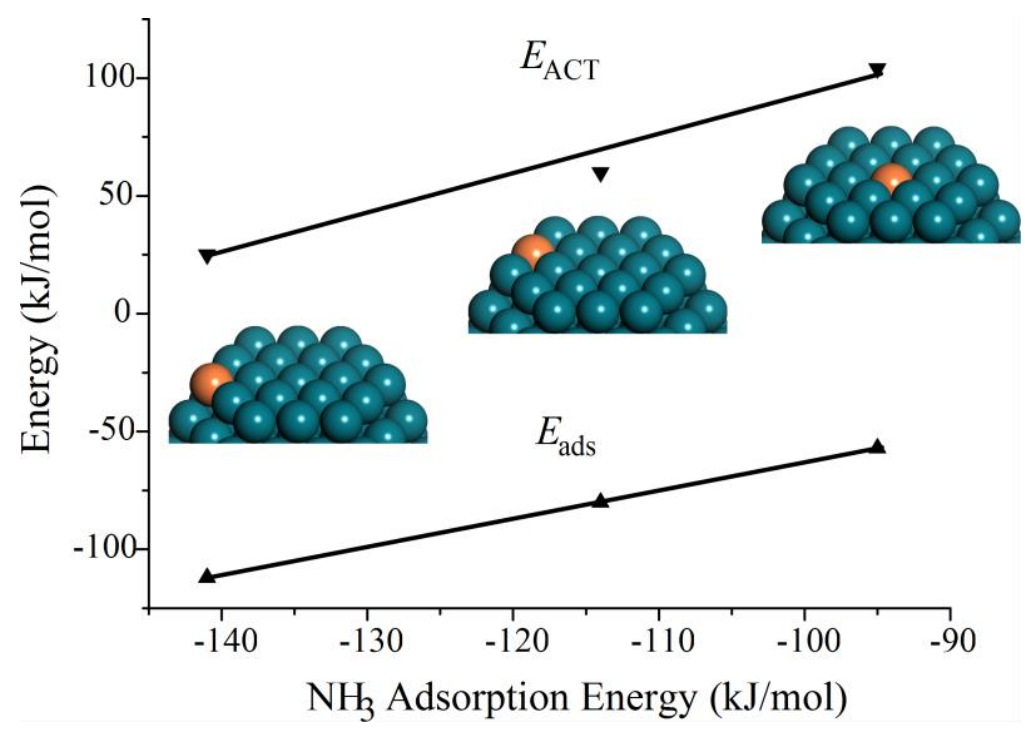

Figure 4.8 Linear correlation of the adsorption energy of THFA and the activation barriers for its ring opening at different $\mathrm{Re}$ sites on the $\mathrm{Rh}$ - $\mathrm{Re}$ particle versus $\mathrm{NH}_{3}$ adsorption energies at these Re sites. 
In order to appropriately compare the energetics for the ring opening of THFA at the three different forms of possible acid sites on the $\mathrm{Rh}-\mathrm{Re}$ alloy particle ( the unoccupied $\mathrm{Re}, \mathrm{Re}-\mathrm{OH}$ and Re- $-\mathrm{OH}_{2}$ ), we must appropriately account for the state of the surface under working conditions. As the reactions are carried out in an aqueous solution, the surface is likely covered with either water or hydroxyl intermediates. We examine detail the state of the surface under working conditions in the next section. For simplicity, we will choose for now a reference state where water is adsorbed to the acid site as the reaction is carried out in aqueous media and THFA is in solution which we have modeled thus far as a gas phase with THFA and water molecules which we can write as: THFA ${ }^{\text {gas }}+\mathrm{Acid}^{\cdots} \mathrm{H}_{2} \mathrm{O}$. As such the reaction at each of the acid sites will require the desorption of water from the site and the adsorption of THFA. The activation energies for the ring opening of THFA at different $\mathrm{Re}-\mathrm{OH}, \mathrm{Re}-\mathrm{OH}_{2}$ and unoccupied Re sites calculated with respect to the reference state defined here $\left(E_{A C T}{ }^{\text {ref }}\right)$ were listed in Table 4.3. We can see that the most active site for the ring opening of THFA is the corner Lewis acid Re site with $E_{A C T}{ }^{\text {ref }}$ at about $-7 \mathrm{~kJ} / \mathrm{mol}$. The edge and corner Brønsted acid $\mathrm{Re}-\mathrm{OH}_{2}$ sites and the edge $\mathrm{Re}-\mathrm{OH}$ acid sites

are also very active for the ring opening of THFA with $E_{A C T}{ }^{r e f}$ at about $-2 \mathrm{~kJ} / \mathrm{mol}, 7 \mathrm{~kJ} / \mathrm{mol}$ and $25 \mathrm{~kJ} / \mathrm{mol}$ respectively.

\subsubsection{Coverage of the unoccupied Re site, $\mathrm{Re}-\mathrm{OH}_{2}$ and Re-OH sites}

Even though we showed above that the unoccupied Re sites, the adsorbed water $\mathrm{Re}-\mathrm{OH}_{2}$ and the adsorbed hydroxyl Re-OH on the Rh-Re alloy surface can all be possible acid sites that can catalyze the ring opening of THFA, the reaction activity also depends on the number of each site on the Rh-Re catalyst under working conditions. Thus here, we try to calculate the coverage of each species at the Re atoms on the Rh-Re surface under the reaction condition at the 
temperature of $393 \mathrm{~K}$ and with $\mathrm{H}_{2}$ pressure of 34 bar. The coverage of these three sites (unoccupied $\mathrm{Re}, \mathrm{Re}-\mathrm{OH}_{2}$ and $\mathrm{Re}-\mathrm{OH}$ ) together with the fully deprotonated Re-O sites are set by the equilibrium reactions shown in Figure 4.9, which include: (1) the adsorption/desorption of $\mathrm{H}_{2} \mathrm{O}$ at the Re sites; (2) the activation of the adsorbed $\mathrm{H}_{2} \mathrm{O}$ to form the adsorbed $\mathrm{OH}$ and gas phase $\mathrm{H}_{2}$; (3) the continuing activation of the adsorbed $\mathrm{OH}$ to form the adsorbed $\mathrm{O}$ and the gas phase $\mathrm{H}_{2}$. The expressions for the coverage of unoccupied $\mathrm{Re}, \mathrm{Re}-\mathrm{OH}_{2}, \mathrm{Re}-\mathrm{OH}$ and $\mathrm{Re}-\mathrm{O}$ sites can be derived as shown in Equation $(4.8 \sim 4.11)$ where the equilibrium constants for each step $\left(K_{i}\right)$ are calculated from the free energy change $\Delta G_{i}$ by Equation 4.12 .

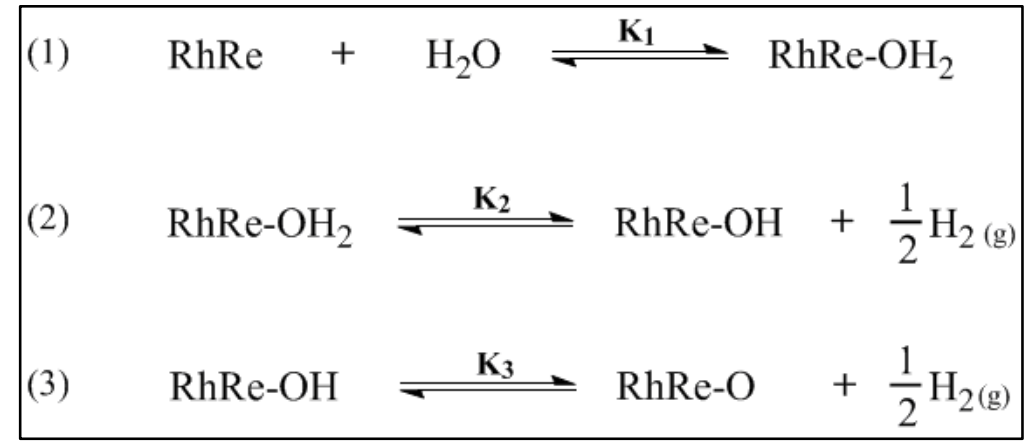

Figure 4.9 Reactions that govern the equilibrium between the unoccupied $\mathrm{Re}$ site, $\mathrm{Re}-\mathrm{OH}_{2}, \mathrm{Re}-$ $\mathrm{OH}$ and $\mathrm{Re}-\mathrm{O}$ sites on the $\mathrm{Rh}-\mathrm{Re}$ surface.

As shown in Table 4.4, in the gas phase, at the temperature of $393 \mathrm{~K}$, the adsorption energy of $\mathrm{H}_{2} \mathrm{O}$ at the $\mathrm{Re}$ atom on the $\mathrm{Rh}$-Re surface is calculated to be $15 \mathrm{~kJ} / \mathrm{mol}$, indicating $\mathrm{H}_{2} \mathrm{O}$ tends not to be adsorbed due to the entropy loss after adsorption. However, this calculation in the gas phase over-predicts the entropy of water in the condensed phase. From the literature, ${ }^{[146]}$ the entropy of water in the condensed phase is about $92 \mathrm{~J} /(\mathrm{mol} \cdot \mathrm{K})$ at $393 \mathrm{~K}$, which is much lower than the entropy of water in the gas phase $(199 \mathrm{~J} /(\mathrm{mol} \cdot \mathrm{K}))$. With the correction of the entropy of water, the adsorption energy of the condensed phase $\mathrm{H}_{2} \mathrm{O}$ at the $\mathrm{Re}$ atom on the $\mathrm{Rh}-\mathrm{Re}$ surface 
$\Delta G_{1}$ is calculated to be $-27 \mathrm{~kJ} / \mathrm{mol}$ at the temperature of $393 \mathrm{~K}$, indicating the condensed-phase water tends to be adsorbed.

$$
\begin{gathered}
{[R h \mathrm{Re}]=\frac{1}{\left(1+K_{1} \times c\left(H_{2} O\right)+K_{1} K_{2} \times \frac{c\left(H_{2} O\right)}{p_{H_{2}}{ }^{0.5}}+K_{1} K_{2} K_{3} \times \frac{c\left(H_{2} O\right)}{p_{H_{2}}}\right)}} \\
{\left[R h \operatorname{Re}-O_{2}\right]=\frac{K_{1} \times c\left(H_{2} O\right)}{\left(1+K_{1} \times c\left(H_{2} O\right)+K_{1} K_{2} \times \frac{c\left(H_{2} O\right)}{p_{H_{2}}{ }^{0.5}}+K_{1} K_{2} K_{3} \times \frac{c\left(H_{2} O\right)}{p_{H_{2}}}\right)}} \\
{[R h \operatorname{Re}-O H]=\frac{K_{1} K_{2} \times \frac{c\left(H_{2} O\right)}{p_{H_{2}}{ }^{2.5}}}{\left(1+K_{1} \times c\left(H_{2} O\right)+K_{1} K_{2} \times \frac{c\left(H_{2} O\right)}{p_{H_{2}}{ }^{0.5}}+K_{1} K_{2} K_{3} \times \frac{c\left(H_{2} O\right)}{p_{H_{2}}}\right)}} \\
{[R h \operatorname{Re}-O]=\frac{\left.K_{1} K_{2} K_{3} \times \frac{c\left(H_{2} O\right)}{p_{H_{2}}}\right)}{\left(1+K_{1} \times c\left(H_{2} O\right)+K_{1} K_{2} \times \frac{c\left(H_{2} O\right)}{p_{H_{2}}{ }^{0.5}}+K_{1} K_{2} K_{3} \times \frac{c\left(H_{2} O\right)}{p_{H_{2}}}\right)}} \\
K_{i}=\exp \left(\frac{-\Delta G_{i}}{R T}\right) \quad(4.12)
\end{gathered}
$$

The free energy change for the activation of the adsorbed water $\mathrm{Re}-\mathrm{OH}_{2}$ to form the adsorbed $\mathrm{OH}$ in the gas phase as the free energy for the dissociation of water $\left(\Delta G_{2}\right)$ is calculated to be $-25 \mathrm{~kJ} / \mathrm{mol}$. The negative value of $\Delta G_{2}$ indicates the equilibrium favors the $\mathrm{Re}-\mathrm{OH}$ rather than $\mathrm{Re}-\mathrm{OH}_{2}$. A more negative value for $\Delta G_{2}(-36 \mathrm{~kJ} / \mathrm{mol})$ is calculated if the reaction is carried out in the condensed water phase as shown in Table 4.4. The difference of $\Delta G_{2}$ between the gas phase and the condensed phase is the result of the different solvation energy of the adsorbed $\mathrm{OH}$ $\left(E_{\text {solv }}(\mathrm{Re}-\mathrm{OH})\right)$ and the adsorbed $\mathrm{H}_{2} \mathrm{O}\left(E_{\text {solv }}\left(\mathrm{Re}-\mathrm{OH}_{2}\right)\right)$ by the water solvent. The difference between the solvation energy of the adsorbed $\mathrm{OH}$ and the adsorbed $\mathrm{H}_{2} \mathrm{O}\left[E_{\text {solv }}(\mathrm{Re}-\mathrm{OH})-E_{\text {solv }}(\mathrm{Re}-\right.$ $\mathrm{OH}_{2}$ )] thus is calculated to be $-11 \mathrm{~kJ} / \mathrm{mol}$, indicating the solvation energy for the Re-OH is larger than the $\mathrm{Re}-\mathrm{OH}_{2}$ in the water solvent. This is likely due to the higher polarity and hydrogen bonding of $\mathrm{OH}$ over that of $\mathrm{H}_{2} \mathrm{O}$. Similarly, the free energy change for the activation of the 
adsorbed $\mathrm{OH}$ to form the adsorbed $\mathrm{O}\left(\Delta G_{3}\right)$ in the gas phase is calculated to be $-8 \mathrm{~kJ} / \mathrm{mol}$ while more positive free energy change $(27 \mathrm{~kJ} / \mathrm{mol})$ is calculated with the water solvent, indicative of the larger solvation energy of the adsorbed $\mathrm{OH}$ than the adsorbed $\mathrm{O}$ by the water solvent due to the increased hydrogen bonding of adsorbed $\mathrm{OH}^{*}$ over that of $\mathrm{O}^{*}$. The difference between the solvation energy of the adsorbed $\mathrm{O}$ and the adsorbed $\mathrm{OH}\left[E_{\text {solv }}(\mathrm{Re}-\mathrm{O})-E_{\text {solv }}(\mathrm{Re}-\mathrm{OH})\right]$ is calculated to be $35 \mathrm{~kJ} / \mathrm{mol}$.

Table 4.4 Free energy changes and equilibrium constants for the reaction steps including the adsorption of $\mathrm{H}_{2} \mathrm{O}$, the activation of $\mathrm{H}_{2} \mathrm{O}^{*}$ to form $\mathrm{OH}^{*}$ and the activation of $\mathrm{OH}^{*}$ to form $\mathrm{O}^{*}$ at the Re atoms on the Rh-Re surface. The units for $\Delta G$ are in $\mathrm{kJ} / \mathrm{mol}$.

\begin{tabular}{lcccccc}
\hline & $\Delta G_{1}$ & $K_{1}$ & $\Delta G_{1}$ & $K_{2}$ & $\Delta G_{1}$ & $K_{3}$ \\
\hline Gas-phase & 15 & - & -25 & - & -8 & - \\
Condensed-phase & -27 & $3.6 \times 10^{3}$ & -36 & $5.4 \times 10^{3}$ & 27 & $2.8 \times 10^{-4}$ \\
\hline
\end{tabular}

The free energy changes for each of the reactions in Figure 4.9 were calculated and along with their corresponding equilibrium constants $K_{1}, K_{2}$ and $K_{3}$ in Table 4.4. By using the value of $K_{1}, K_{2}$ and $K_{3}$ and the concentration of condensed phase water $c\left(\mathrm{H}_{2} \mathrm{O}\right)=1$ and the $\mathrm{H}_{2}$ pressure $p_{\mathrm{H} 2}=34$ bar along with Equations 4.8 4.11, we can solve for the surface coverages of the unoccupied Re, Re- $\mathrm{OH}_{2}$, Re-OH and Re-O sites on the Rh-Re alloy surface as show in Table 4.5. The results indicate that the Re sites within the Rh-Re alloy surface are predominantly covered by $\mathrm{OH}^{*}$ as the coverage of $\mathrm{OH}^{*}$ was calculated to be $\sim 1$ while the coverages of the other sites were very small. 
Table 4.5 Coverage of the unoccupied Re site, adsorbed water, adsorbed hydroxyl and adsorbed oxygen at the Re atoms on the Rh-Re surface.

\begin{tabular}{cccc}
\hline Unoccupied Re & Re-OH & Re-OH & Re-O \\
\hline $3.0 \times 10^{-8}$ & $1.1 \times 10^{-4}$ & 1.00 & $4.8 \times 10^{-5}$ \\
\hline
\end{tabular}

The coverages for the different $\mathrm{OH}_{\mathrm{x}}$ intermediates at each of the different sites Re sites (terrace, edge and corner) within the Rh-Re particle surface were also analyzed. The large computational cost prohibited the simulations of the 201 metal particles in an aqueous solution. The simulations for the Rh-Re particle were carried out instead in the gas phase. If we assume that the solvation energies of the $\mathrm{Re}-\mathrm{OH}_{2}, \mathrm{Re}-\mathrm{OH}$ and $\mathrm{Re}-\mathrm{O}$ at different $\mathrm{Re}$ atoms (terrace, edge and corner) on the Rh-Re particle surface do not change with the location of the Re atom, we can then readily calculate the free energy changes for reaction (2) and reaction (3) (Figure 4.9) in water by adding the appropriate solvation energy contributions $\left[E_{\text {solv }}(\mathrm{OH})-E_{\text {solv }}\left(\mathrm{H}_{2} \mathrm{O}\right)\right]=-11$ $\mathrm{kJ} / \mathrm{mol}$ or $\left[E_{\text {solv }}(\mathrm{O})-E_{\text {solv }}(\mathrm{OH})\right]=35 \mathrm{~kJ} / \mathrm{mol}$ reported above to the calculated gas-phase free energy changes. As shown in Table 4.6, the free energy changes for the activation of the adsorbed $\mathrm{H}_{2} \mathrm{O}$ to form the adsorbed $\mathrm{OH}$ at the terrace, edge and corner $\mathrm{Re}$ atoms on the Rh-Re particle are all negative, indicating the $\mathrm{Re}-\mathrm{OH}$ species that forms is thermodynamically the favorable state. The free energy changes involving the activation of the adsorbed $\mathrm{OH}$ to adsorbed $\mathrm{O}$ at the terrace, edge and corner $\mathrm{Re}$ atoms on the $\mathrm{Rh}$-Re particle in the water solvent were all calculated to be slightly positive, thus indicating the Re-O is not as favorable as $\mathrm{Re}-\mathrm{OH}$ in the water solvent. 
Table 4.6 Free energy changes calculated in gas phase and in water solvent for the adsorption of $\mathrm{H}_{2} \mathrm{O}$, the activation of $\mathrm{H}_{2} \mathrm{O}^{*}$ to form $\mathrm{OH}^{*}$ and the activation of $\mathrm{OH}^{*}$ to form $\mathrm{O}^{*}$ at the $\mathrm{Re}$ atoms on the Rh-Re particle with Re atoms located at the terrace, edge and corner site. The units are in $\mathrm{kJ} / \mathrm{mol}$.

\begin{tabular}{lcccccc}
\hline & \multicolumn{2}{c}{$\Delta G_{1}$} & \multicolumn{2}{c}{$\Delta G_{2}$} & \multicolumn{2}{c}{$\Delta G_{3}$} \\
\hline & Gas-phase & Water solvent & Gas-phase & Water solvent & Gas-phase & Water solvent \\
\hline Terrace & 25 & -16 & -11 & -22 & -29 & 6 \\
Edge & 11 & -31 & -72 & -83 & -33 & 2 \\
Corner & -13 & -55 & -111 & -122 & -34 & 1 \\
\hline
\end{tabular}

The free energy changes were used to determine the equilibrium constants $K_{1}, K_{2}$ and $K_{3}$ reported in Table 4.7, which were subsequently used to calculate the coverage of the unoccupied Re site, $\mathrm{Re}-\mathrm{OH}_{2}, \mathrm{Re}-\mathrm{OH}$ and Re-O sites. The results in Table 4.8 show that at the temperature of $393 \mathrm{~K}$ and $\mathrm{H}_{2}$ pressure of 34 bar, the Re atoms at the terrace, edge and corner sites of the Rh-Re particle are predominantly covered by $\mathrm{OH}$ with about $0.96 \mathrm{ML}$ coverage at the terrace $\mathrm{Re}$ atoms, 0.91 ML coverage at the edge Re atoms and about $0.88 \mathrm{ML}$ coverage at the corner Re atoms. Some Re atoms are covered by oxygen with about 0.03 coverage at the terrace Re atoms, and about 0.09 coverage and 0.12 coverage at the edge and corner Re atoms respectively. The amounts of the unoccupied Re site and the adsorbed water are almost zero. 
Table 4.7 Equilibrium constants calculated in water solvent for the adsorption of $\mathrm{H}_{2} \mathrm{O}$, the activation of $\mathrm{H}_{2} \mathrm{O}^{*}$ to form $\mathrm{OH}^{*}$ and the activation of $\mathrm{OH}^{*}$ to form $\mathrm{O}^{*}$ at the $\mathrm{Re}$ atoms on the $\mathrm{Rh}-\mathrm{Re}$ particle with Re atoms located at the terrace, edge and corner site.

\begin{tabular}{lccc}
\hline & $K_{1}$ & $K_{2}$ & $K_{3}$ \\
\hline Terrace & $1.4 \times 10^{2}$ & $8.2 \times 10^{2}$ & $1.7 \times 10^{-1}$ \\
Edge & $1.2 \times 10^{4}$ & $7.4 \times 10^{10}$ & $5.6 \times 10^{-1}$ \\
Corner & $1.6 \times 10^{7}$ & $1.2 \times 10^{16}$ & $8.1 \times 10^{-1}$ \\
\hline
\end{tabular}

Table 4.8 Coverage of unoccupied Re, adsorbed water, adsorbed hydroxyl and adsorbed oxygen at the different $\mathrm{Re}$ atoms on the Rh-Re particle.

\begin{tabular}{lcccc}
\hline & Unoccupied Re & $\mathrm{Re}^{-O H_{2}}$ & $\mathrm{Re}-\mathrm{OH}$ & $\mathrm{Re}-\mathrm{O}$ \\
\hline Terrace & $4.8 \times 10^{-5}$ & $6.9 \times 10^{-3}$ & 0.96 & 0.03 \\
Edge & $5.8 \times 10^{-15}$ & $7.2 \times 10^{-11}$ & 0.91 & 0.09 \\
Corner & $2.6 \times 10^{-23}$ & $4.2 \times 10^{-16}$ & 0.88 & 0.12 \\
\hline
\end{tabular}

As the Re atoms on the surface of the Rh-Re particle are mainly covered by the hydroxyl species and the number of the unoccupied Re atom and the adsorbed $\mathrm{H}_{2} \mathrm{O}$ are very low, the Re$\mathrm{OH}$ sites are the most plausible active acid sites that catalyze the ring opening of cyclic ethers and polyols such as THFA as we showed in Chapter 3.

\subsection{Conclusions}

Detailed x-ray analyses reported in the literature resulted in different speculations as to the nature of the acid site in the Re-promoted $\mathrm{Rh}$ and Pt systems. In this chapter, we examined properties and the reactivity of unoccupied Re sites which serve as Lewis acids as well as Brønsted acid sites established via the binding or $\mathrm{H}_{2} \mathrm{O}$ or $\mathrm{OH}$ to the Re sites in the $\mathrm{C}-\mathrm{O}$ activation of THFA and other cyclic ethers and polyols on the Rh-Re alloy surface in aqueous systems. 
The results indicate that the adsorbed water $\left(\mathrm{Re}-\mathrm{OH}_{2}\right)$ can behave as Brønsted acid sites analogous to Re-OH sites that we reported previously. The acidities of the edge and corner Re$\mathrm{OH}_{2}$ sites are even stronger than the Re-OH sites with lower deprotonation energies. The DPE of the adsorbed water at the terrace, edge and corner Re atoms were found to be linearly correlated to the difference between the binding energy of $\mathrm{OH}$ and $\mathrm{H}_{2} \mathrm{O}$ at these Re atoms $\left[\Delta E_{\text {ads }}(\mathrm{OH})\right.$ $\left.\Delta E_{a d s}\left(\mathrm{H}_{2} \mathrm{O}\right)\right]$.

$\mathrm{NH}_{3}$ was also shown to strongly adsorb to the free $\mathrm{Re}$ atoms on the $\mathrm{Rh}-\mathrm{Re}$ surface with binding energies of $-95,-114$ and $-141 \mathrm{~kJ} / \mathrm{mol}$ at the terrace, edge and corner Re atom sites, respectively. The strong binding of $\mathrm{NH}_{3}$ to the $\mathrm{Re}$ atoms on the $\mathrm{Rh}$ - $\mathrm{Re}$ alloy surface and the Brønsted acidity of the adsorbed $\mathrm{H}_{2} \mathrm{O}$ and $\mathrm{OH}$ at the Re atoms on the $\mathrm{Rh}$-Re alloy surface suggests the Re atoms on the Rh-Re alloy surface have Lewis acid character. A detailed charge analysis of the Re atoms on the Rh-Re alloy surface showed that even the metallic Re atoms without adsorbed $\mathrm{H}_{2} \mathrm{O}$ or $\mathrm{OH}$ are positively charged. The adsorption of $\mathrm{H}_{2} \mathrm{O}$ or $\mathrm{OH}$ at the $\mathrm{Re}$ atoms increase the positive charge on the Re atoms. The partial positive charge on the metallic Re atoms on the Rh-Re alloy surface suggests that the low positive oxidation state measured by the experiments does not necessarily indicate the formation of oxide of Re on the Rh-Re catalyst.

The ring opening of the cyclic ether THFA at the $\mathrm{Re}-\mathrm{OH}_{2}$ sites on the Rh-Re alloy surface were found to proceed via the same mechanism reported previously at the $\mathrm{Re}-\mathrm{OH}$ sites, which involves the concerted addition of the proton to the oxygen, heterolytic $\mathrm{C}-\mathrm{O}$ activation ring and $\mathrm{H}$ transfer from the $\mathrm{C} 1$ to the $\mathrm{C} 2$ site to form an oxocarbenium ion intermediate. The activation barrier for the ring opening of THFA at the terrace $\mathrm{Re}-\mathrm{OH}_{2}$ site was calculated to be $98 \mathrm{~kJ} / \mathrm{mol}$, while much lower energy barriers were calculated for the ring opening of THFA at the edge and corner Re- $\mathrm{OH}_{2}$ sites with the energy barrier of $17 \mathrm{~kJ} / \mathrm{mol}$ and $28 \mathrm{~kJ} / \mathrm{mol}$ respectively. The lower 
activation barriers for the ring opening of THFA at the edge and corner $\mathrm{Re}-\mathrm{OH}_{2}$ site are the result of the higher acidity of these two acid sites as well as the involvement of neighboring coordinatively-unsaturated $\mathrm{Rh}$ metal atoms in stabilizing the molecular $\mathrm{OH}$ in the transition state for these two acid sites.

The ring opening of THFA at the uncovered Re atoms on the Rh-Re alloy surface also proceeds via the concerted ring opening and $\mathrm{H}$-transfer mechanism, similar to that at the Brønsted acid sites $\mathrm{Re}-\mathrm{OH}_{2}$ and $\mathrm{Re}-\mathrm{OH}$. The charge analysis showed that a negative charged center on the metal particle and a positive charged center on the carbenium ion are formed in the transition state, which is very similar to the transition states of other typical Lewis acid catalyzed ring opening of epoxides. It is found that both the adsorption energy of THFA and the energy barriers for the ring opening of THFA at the terrace, edge and corner Re atoms on the Rh-Re particle can be linearly correlated to the binding energy of $\mathrm{NH}_{3}$ at these different $\mathrm{Re}$ atoms, which can be a measurement of the acidity of Lewis acids.

Even though the adsorbed $\mathrm{H}_{2} \mathrm{O}, \mathrm{OH}$ and uncovered Re atoms on the Rh-Re alloy surface can all be possible active sites that catalyze the ring opening of THFA, the reaction rates will depend on the number of these active sites on the $\mathrm{Rh}$-Re catalysts under working catalytic conditions. DFT results indicate that for reactions carried out at $393 \mathrm{~K}$ and $\mathrm{H}_{2}$ pressures of 34 bar in the aqueous solution, the Re atoms on the Rh-Re alloy particle surface are dominantly covered by the hydroxyl group with $<1 \%$ adsorbed $\mathrm{H}_{2} \mathrm{O}$ and unoccupied Re sites. Thus, the adsorbed $\mathrm{OH}$ at the $\mathrm{Re}$ atoms on the $\mathrm{Rh}-\mathrm{Re}$ alloy surface are likely active acid sites that catalyze the ring opening of THFA and other cyclic ethers and polyols over the Rh-Re catalysts. 


\section{Chapter 5-Entropy Effects on Dissociation of Water and Hydroxyl, and Ring Opening of THFA over the Rh-Re Surface in Aqueous Solution}

\subsection{Introduction}

In the previous chapters, we have examined in some detail the nature of the metal and acid sites, their properties and the governing pathways and potential mechanisms for hydrogenolysis of cyclic ethers and polyols. Our focus was on the changes in the activation barrier and overall reaction energies. We noted that the solution phase plays an active role in the chemistry and the calculated energetic but did not explore in the effects of the solution and the stability of the active sites or the influence of entropy in both the formation of the active sites and in the actual catalytic transformations. These effects are important and will be analyzed in more detailed in this Chapter.

In Chapters 3 and 4, we showed that the active sites for the hydrogenolysis of cyclic ethers and polyols likely involves the presence of the active metal for hydrogenation (Rh) and oxophilic metal $(\mathrm{Re})$ to produce an active acid site which we believe is of the form $\mathrm{Re}-\mathrm{OH}$. In aqueous or protic media, unlike in the gas phase or in the hydrocarbon solvent, Brønsted acids dissociate to form a proton which is typically in the form of a hydronium ion and the conjugate base. The proton which forms can then carry out acid catalyzed chemistry. Similar to the homogeneous Brønsted acid systems, the solid acids can also dissociate the $\mathrm{O}-\mathrm{H}$ bond to form the solvated hydronium ions in water. Previous IR spectra by Lercher et al. ${ }^{[147]}$ show that hydronium ions are formed when water is adsorbed over the ZSM-5 zeolite with higher molar ratio than the zeolitic protons as the IR spectra show bands at 2885 and $2463 \mathrm{~cm}^{-1}$ which are characteristic for hydronium ions. The X-ray and neutron diffraction data by Brown et al. ${ }^{[148]}$ also show $\mathrm{H}_{3} \mathrm{O}^{+}$or $\mathrm{H}_{5} \mathrm{O}_{2}{ }^{+}$ions are generated when water is assimilated by solid heteropolyacid catalysts. The 
dissociation of the solid acids raises the question of whether the bound proton or the solution phase proton carries out catalysis and will influence the activity for reactions that need to occur on the surface of the catalyst. Dumesic et al. ${ }^{[72]}$ have shown that the hydrogenolysis of cyclic ether THFA over the $\mathrm{Rh} / \mathrm{C}$ catalysts in the presence of homogeneous acids such as $\mathrm{HCl}$ or $\mathrm{H}_{2} \mathrm{SO}_{4}$ is over 10x times lower in activity compared to that over the Rh-Re catalysts, which suggests it is the surface acid site on the Rh-Re catalysts rather than the hydronium ion in the solution are required for the ring opening of THFA. Thus to better understand the nature of the acid sites on the Rh-Re catalysts in water solution, the dissociation of the Re-OH acid site on the Rh-Re surface in water solution is studied here.

In the previous two chapters, we also showed that water readily activates at the Re atoms on the Rh-Re alloy surface to form an adsorbed $\mathrm{OH}$ that then function as Brønsted acid site. Desai and Neurock ${ }^{[128]}$ previously showed that the activation of water in an aqueous media on a bifunctional alloy surface such as Pt-Ru surface likely proceeds via a heterolytic path to form the adsorbed hydroxyl intermediates on the surface and protons in the solution. Thus here, we want to understand the heterolytic dissociation of water as well as surface hydroxyl groups on the RhRe surface in solution. It is important to note that similar reactions occur in electrocatalytic reactions such as the oxidation of $\mathrm{CO}^{[128]}$ and hydrogen evolution ${ }^{[149]}$ that occur on the electrode surface and thus may provide important insights in the nature of the active sites and their stability. Previous studies on the activation of water carried out over a metal surface in the presence of solution ${ }^{[128]}$ indicate that the reaction proceeds via the heterolytic dissociation of water but do not consider the changes in entropy that can occur or the overall free energy of the reaction. Herein we use constrained molecular dynamics simulation to calculate the free energy for the activation of water and hydroxyl at the Re atoms on the Rh-Re alloy surface to assess the 
influence of entropy and reaction conditions.

In Chapter 3, we demonstrated that water helped to promote the ring-opening of THFA at an acid site on the Rh-Re carried out by stabilizing the transition state and lower the activation barrier for ring opening. As this reaction requires the reorganization of the water solvent, the entropic changes may be just as important as the enthalpic changes. We use constrained molecular dynamics simulation methods here to analyze the ring opening of THFA at the acid site on the Rh-Re surface in water solution to further understand this reaction with entropy changes considered.

\subsection{Computational Methods}

All of the calculations reported herein were carried out using periodic plane-wave gradientcorrected density functional theory methods implemented in the Vienna ab initio Simulation Package (VASP). ${ }^{[89-92]}$ The PW91 ${ }^{[93]}$ form of GGA exchange correlation functional was used to determine the non-local gradient-corrections to exchange and correlation energies. Wave functions were constructed by using projector augmented wave potentials (PAW) ${ }^{[94,95]}$ within a cutoff energy of $396.0 \mathrm{eV}$. For all structure optimizations, the electronic energies were converged to within $10^{-6} \mathrm{eV}$ and the forces on each atom were optimized to within $0.05 \mathrm{eV} \AA^{-1}$. A 6x6x1 Monkhorst-Pack ${ }^{[96]}$ k-point mesh was used to sample the first Brillouin zone.

In the molecular dynamics simulation, the electronic energies were converged to within $10^{-4}$ using a $3 \times 3 \times 1$ Monkhorst-Pack ${ }^{[96]}$ k-point mesh. The NVT ensemble was used in the molecular dynamics simulations. The temperature of $300 \mathrm{~K}$ was controlled using a Nose-Hoover thermostat. ${ }^{[114,115]}$ An integration step of $1 \mathrm{fs}$ was used. The atomic mass of tritium was used for all $\mathrm{H}$ atoms to avoid thermal separation of 'hard' modes such as $\mathrm{C}-\mathrm{H}$ and $\mathrm{O}-\mathrm{H}$ stretching modes 
in the molecular dynamics simulations. ${ }^{[150]}$

The $\mathrm{Rh}-\mathrm{Re}$ alloy surface was modeled by substituting a $\mathrm{Rh}$ atom in the $\mathrm{Rh}(111)$ surface with Re. For the simulations of the dissociation of water and hydroxyl, the Rh(111) surface was modeled using a $3 \times 3$ unit cell with four metal layers and a $15 \AA$ vacuum region above the metal surface. The solution phase above the metal surface was modeled by filing 24 water molecules randomly into the vacuum region resulting in a density of water that is close to $1 \mathrm{~g} / \mathrm{cm}^{3}$.

The simulation for the ring opening of THFA at the Re-OH acid site on the Rh-Re surface required a large unit cell to space the THFA molecules further apart. As such, these simulations were carried out using a 4x4 unit cell with only two metal layers and a $15 \AA$ vacuum region above the metal layers. The top layer was allowed to relax and the bottom layer held fixed to their lattice constant for Rh. The acid site was modeled by substituting one of the $\mathrm{Rh}$ atoms in the $\mathrm{Rh}(111)$ with a $\mathrm{Re}-\mathrm{OH}$ site. The solution phase above the Rh surface was modeled by filling 40 water molecules randomly in the vacuum region so that the density of water is close to 1 $\mathrm{g} / \mathrm{cm}^{3}$. Three water molecules around the acid site are removed to allow space to put the adsorbate such as THFA. The structural optimizations with solution phase were carried out after molecular dynamics simulations at $400 \mathrm{~K}$ for 2 ps to equilibrate the water system.

Transition state searches were performed using the dimer method ${ }^{[98]}$ and the nudged elastic band (NEB) method ${ }^{[97]}$ as introduced in Chapter 2. Charge analyses were performed by using the QUAMBO method ${ }^{[99-102]}$ as also introduced in Chapter 2.

Constrained ab initio molecular dynamics were carried out using the SHAKE algorithm. ${ }^{[151]}$ In this algorithm, the Lagrangian for the system is extended as shown in Equation 5.1.

$$
L=L_{0}+\sum_{i=1}^{r} \lambda_{i} \sigma_{i}(q)
$$

where the summation is over geometric constraints and $\lambda_{i}$ is a Lagrange multiplier associated 
with a geometric constraint $\sigma_{i}$ defined in Equation 5.2:

$\sigma_{i}(q)=\xi_{i}(q)-\xi_{i}$

with $\xi_{i}(q)$ being a geometric parameter and $\xi_{i}$ is the value of $\xi_{i}(q)$ fixed during the simulation. In the SHAKE algorithm, ${ }^{[151]}$ Lagrangian multipliers $\lambda_{i}$ can be determined in an iterative procedure

In general, the constrained molecular dynamics generates biased statistical averages. It can be shown that the correct average for a quantity $a(\xi)$ can be obtained using the Equation 5.3. ${ }^{[152-}$ 155]

$a(\xi)=\frac{\left\langle|Z|^{-1 / 2} a\left(\xi^{*}\right)\right\rangle_{\xi^{*}}}{\left\langle|Z|^{-1 / 2}\right\rangle_{\xi^{*}}}$

where $\langle\ldots\rangle_{\xi}^{*}$ stands for the statistical average of the quantity enclosed in angular parentheses computed for a constrained ensemble and $Z$ is a mass metric tensor defined as:

$Z=\sum_{i=1}^{3 N} m_{i}^{-1} \nabla_{i} \xi_{\alpha} \cdot \nabla_{i} \xi_{\beta}, \alpha=1, . .2, . . r, \beta=1, . .2, . . r$

Thus, the free energy gradient or mean force can be computed using the equation: ${ }^{[152-155]}$

$\left\langle\frac{\partial A}{\partial \xi}\right\rangle_{\xi^{*}}=\frac{1}{\left\langle Z^{-1 / 2}\right\rangle_{\xi^{*}}}\left\langle Z^{-1 / 2}\left[-\lambda_{k}+\frac{k_{B} T}{2|Z|} \sum_{j=1}^{r}\left(Z^{-1}\right)_{k j} \sum_{i=1}^{3 N} m_{i}^{-1} \nabla_{i} \xi_{j} \cdot \nabla_{i}|Z|\right]\right\rangle_{\xi^{*}}$

where $\lambda_{k}$ is the Lagrange multiplier associated with the parameter $\xi$ used in the SHAKE algorithm.

The free energy profile for a reaction can be calculated by the thermodynamic integration method ${ }^{[154]}$ by integrating the mean forces with respect to the reaction coordinate along the reaction path as shown in Equation 5.6., The constrained molecular dynamics simulations were carried out with different fixed values of $\xi$ for 5 ps with the time step of 1 fs. The simulations were run 2 ps to equilibrate the system first, the mean forces at each of the fixed values of $\xi$ were 
then calculated after continuing the simulations to 3 additional picoseconds. The free energy difference $\Delta A$ given by integral in Equation 5.6 was calculated by using the trapezoid rule as shown in Equation 5.7. The static error of $\Delta A$ due to the convergence of the mean force $\epsilon_{\Delta \mathrm{A}}$ can then be calculated by Equation (5.8).

$$
\begin{aligned}
& \Delta A_{1 \rightarrow 2}=\int_{\xi(1)}^{\xi(2)}\left(\frac{\partial A}{\partial \xi}\right)_{\xi^{*}} d \xi \quad(5.6) \\
& \Delta A=\frac{1}{2} f(0) \cdot(\xi(1)-\xi(0))+\frac{1}{2} \sum_{i=1}^{N-1} f(i) \cdot(\xi(i+1)-\xi(i))+\frac{1}{2} f(N) \cdot(\xi(N)-\xi(N-1))+\mathrm{O}\left(\Delta \xi^{2}\right)
\end{aligned}
$$

$$
\varepsilon_{\Delta A}=\frac{1}{2} \varepsilon_{f(0)} \cdot|\xi(1)-\xi(0)|+\frac{1}{2} \sum_{i=1}^{N-1} \varepsilon_{f(i)} \cdot|\xi(i+1)-\xi(i)|+\frac{1}{2} \varepsilon_{f(N)} \cdot|\xi(N)-\xi(N-1)|
$$

The constrained molecular dynamics simulations were performed at a constant volume rather than at a constant pressure in order to obtain the Helmholtz rather than the Gibbs free energies. For condensed phase systems, the difference between the two should be very small.

\subsection{Results and Discussions}

\subsubsection{Dissociation of water and hydroxyl at the Re atom on Rh-Re alloy surface}

The structures and calculated energies for the dissociation of the adsorbed water at the Re sites on the Rh-Re surface was shown in Figure 5.1. We can see that in the reactant state, the adsorbed water covalently binds to the Re via its oxygen and, in addition, forms hydrogen bonds to neighboring water molecules in the solution. The reaction proceeds via the heterolytic cleavage of the $\mathrm{O}-\mathrm{H}$ bond via proton-coupled electron transfer path where the electrons from the

metal are donated into an anitbonding $\sigma_{\mathrm{O}-\mathrm{H}}{ }^{*}$ bond while the proton is transferred to the oxygen of 
vicinal water molecule in solution to which it was hydrogen-bonded. The solution phase water molecule in turn transfers one of its protons to the next adjacent water molecule. The transition state structure shown in Figure 5.1 is very similar to that is reported for the water heterolytic dissociation on Pt-Ru alloy surface in water solution. ${ }^{[128]}$

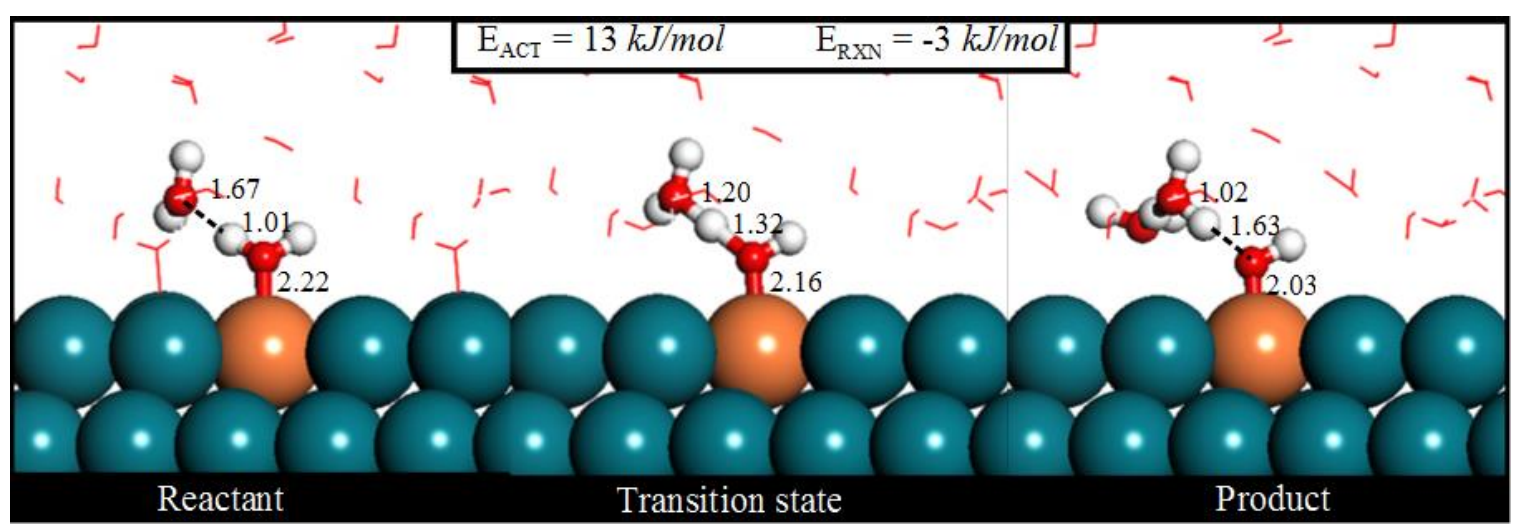

Figure 5.1 Static DFT calculated reactant, transition state and product structures for the dissociation of the adsorbed water at the Re site on the Rh-Re surface in water solvent.

A detailed charge analysis of the reactant, transition state and product structures for the water dissociation process is reported in Table 5.1 and Figure 5.2. As the reaction proceeds, there is an increase in the negative charge that results on the hydroxyl intermediate that forms to the $\mathrm{Re}$ as the reaction proceeds. The charge on the $\mathrm{OH}$ in the adsorbed water reactant $(-0.28)$ increases to -0.33 in the transition state and finally to -0.37 in the product state. The negative charge on the metal surface $\left(\mathrm{Rh}_{35} \mathrm{Re}\right)$ also increases from -0.28 in the reactant state to -0.32 in the transition state and -0.40 in the product state. The dissociation of water thus results in the formation of a positively charge hydronium ion in the solution and the negatively charged surface hydroxyl intermediate in which the charge is located on both the metal as well as on the hydroxyl. 
Table 5.1 Charge analyses of the reactant, transition state and product for the dissociation of the adsorbed water at the Re site on the Rh-Re surface in water solvent.

\begin{tabular}{lccc}
\hline & Reactant & Transition state & Product \\
\hline $\mathrm{OH}^{*}$ & -0.28 & -0.33 & -0.37 \\
$\mathrm{Rh}_{35} \mathrm{Re}$ & -0.28 & -0.32 & -0.40 \\
$\mathrm{Rh}_{35} \mathrm{Re}-\mathrm{OH}$ & -0.56 & -0.65 & -0.77 \\
\hline
\end{tabular}
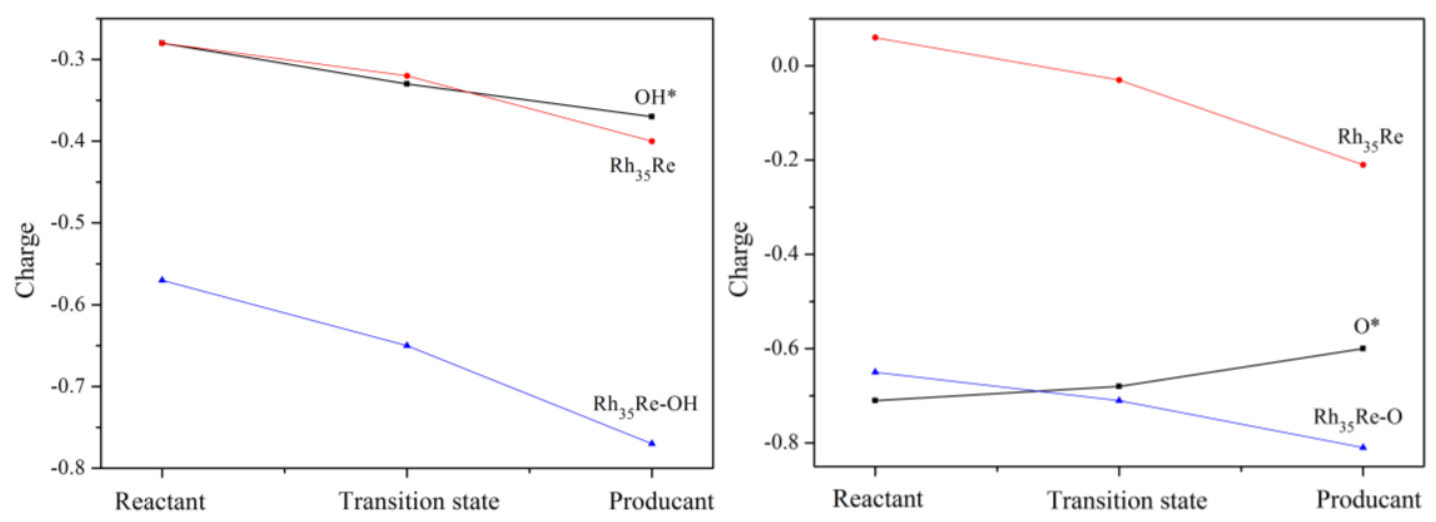

Figure 5.2 Charge changes from the reactant to the transition state and product for the dissociation of the adsorbed water (left) and hydroxyl (right) at the Re sites on the Rh-Re surface in water solvent.

The activation barrier for this heterolytic dissociation path of water was calculated to be 13 $\mathrm{kJ} / \mathrm{mol}$ and the reaction energy is calculated to be slightly exothermic at $-3 \mathrm{~kJ} / \mathrm{mol}$. The much lower energy barrier for this heterolytic activation path than the metal insertion path showed in Chapter $3(54 \mathrm{~kJ} / \mathrm{mol})$ for the activation of water at the Re sites on the $\mathrm{Rh}-\mathrm{Re}$ surface indicates that the heterolytic path is much more favorable. This is consistent with previous simulation results for the activation of water on the Pt-Ru alloy surface. ${ }^{[128]}$

The dissociation of the adsorbed hydroxyl at the solution interface proceeds in a very similar manner as the activation of the $\mathrm{O}-\mathrm{H}$ proceeds heterolytically via a proton-coupled electron transfer reaction resulting in the formation of the $\mathrm{H}_{2} \mathrm{O}_{5}{ }^{+}$Zundel ion intermediate and a 
negatively charged $\mathrm{O} *$ bound to $\mathrm{Re}$ in the $\mathrm{Rh}$-Re surface as is shown in Figure 5.3. The transition state structure is similar to that for the dissociation of water as shown above in Figure 5.1 that covalently bonded hydrogen atom of the adsorbed hydroxyl transfers as proton to an oxygen atom on a vicinal water molecule in the solution thus resulting in a negatively charged $\mathrm{Re}-\mathrm{O}$ surface intermediate.

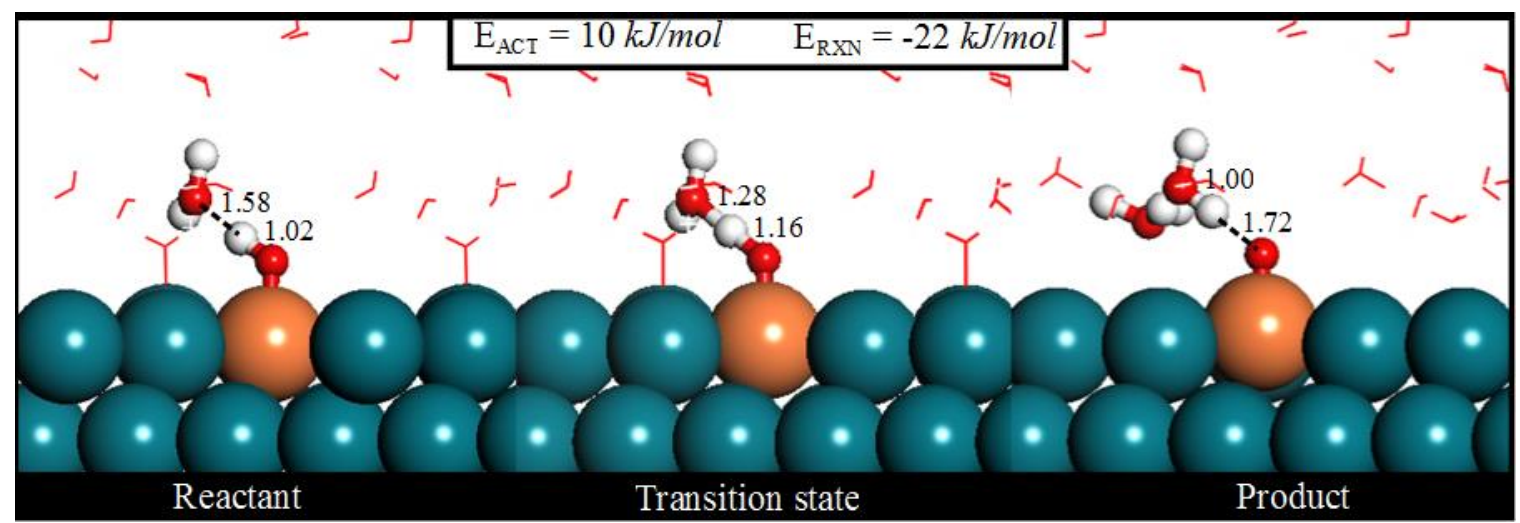

Figure 5.3 Static DFT calculated reactant, transition state and product structures for the dissociation of the adsorbed hydroxyl at the Re site on the Rh-Re surface in water solvent.

A detailed charge analysis for the reactant state, transition state and product state reported in Table 5.2 and Figure 5.2 shows that the negative charge on the metal surface $\left(\mathrm{Rh}_{35} \mathrm{Re}\right)$ increases from +0.06 in the reactant state to -0.03 in the transition state and -0.21 in the product state which is similar to that seen for the activation of water. The change in the charge on the $\mathrm{O}^{*}$ is quite different than that which was found on the $\mathrm{OH}^{*}$. The negative charge was found to increase on the $\mathrm{OH}$ that forms during the activation of water from -0.28 for the reactant to -0.33 for the transition state to -0.37 for the product state, while the negative charge on $\mathrm{O}^{*}$ was found to decrease from -0.71 in the reactant state to -0.68 in the transition state and -0.60 in the product state. These differences are due to the fact that $\mathrm{O}^{*}$ can only withdraw electrons from the metal 
surface where $\mathrm{OH}^{*}$ can withdraw as well as donate electrons to the metal surface. Thus, after the dissociation of the adsorbed hydroxyl, while the positive charge is on the hydronium ions in the solution, the negative charge is located only on the metal surface, but not on the oxygen that is adsorbed on the metal surface.

Table 5.2 Charge analyses of the reactant, transition state and product for the dissociation of the adsorbed hydroxyl at the Re site on the Rh-Re surface in water solvent.

\begin{tabular}{lccc}
\hline & Reactant & Transition state & Product \\
\hline $\mathrm{O}^{*}$ & -0.71 & -0.68 & -0.60 \\
$\mathrm{Rh}_{35} \mathrm{Re}$ & +0.06 & -0.03 & -0.21 \\
$\mathrm{Rh}_{35} \mathrm{Re}-\mathrm{O}$ & -0.65 & -0.71 & -0.81 \\
\hline
\end{tabular}

The activation barrier for the dissociation of $\mathrm{Re}-\mathrm{OH}$ was calculated to be $10 \mathrm{~kJ} / \mathrm{mol}$, which is slightly lower than the activation of water $(13 \mathrm{~kJ} / \mathrm{mol})$. The reaction energy was calculated here to be more exothermic at about $-22 \mathrm{k} / \mathrm{mol}$. The lower activation barrier along with the increased exothermicity for the dissociation of the hydroxide over that for the dissociation of water is consistent with the stronger acidity of the adsorbed hydroxyl than the adsorbed water at the terrace Re sites on the Rh-Re particle as was reported in Chapter 4.

\subsubsection{Entropy effects on the dissociation of water and hydroxyl on Rh-Re surface}

Constrained ab initio molecular dynamics (AIMD) simulations were carried out on the dissociation of the adsorbed water and hydroxyl on the Rh-Re surface to quantify the changes in entropy and free energy between the transition and the reactant states as well as the product and the reactant states. As we found in the static simulations, the dissociation of water proceeds via 
the heterolytic activation of the $\mathrm{O}-\mathrm{H}$ bond via a PCET process where the proton transfers to a vicinal water molecule in solution to form a hydroneium ion intermediate along with the formation of a negatively charged $\mathrm{OH}^{*}$ intermediate, we use $\xi=r(\mathrm{O} 1-\mathrm{H})-r(\mathrm{O} 2-\mathrm{H})$ to represent the constrained reaction coordinate in the constrained AIMD simulations as shown in Figure 5.4. The static DFT optimized structure for non-dissociated water structure with the value of $\xi$ at 0.66 $\AA(r(\mathrm{O} 1-\mathrm{H} 1)=1.67 \AA$ and $r(\mathrm{O} 2-\mathrm{H} 1)=1.01 \AA)$ was used to start the simulations and was taken as the zero point in the free energy profile. We can see in Figure 5.5 that the mean force on the constraint is almost zero, indicating this structure corresponding to a potential-energy minimum is also close to the reactant minimum on the free energy surface. The dissociation reaction was subsequently induced by carrying out dynamics simulations at constraint to fixed values of $\xi$ along the reaction coordinate ranging from $0.66 \AA, 0.51 \AA, 0.35 \AA, 0.21 \AA, 0.07 \AA,-0.07 \AA$, $0.21 \AA,-0.35 \AA,-0.51 \AA$ and $-0.64 \AA$.

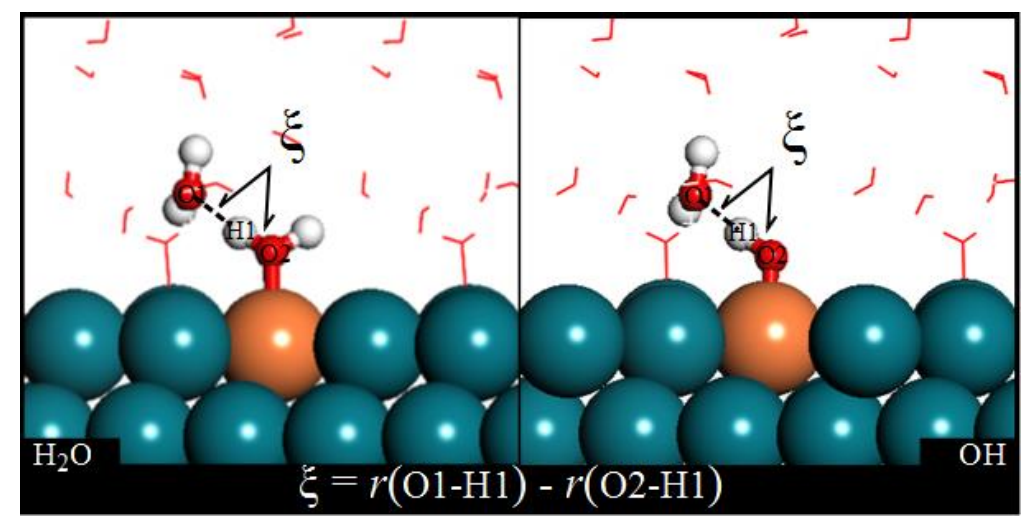

Figure 5.4 Constrained reaction coordinates for the constrained molecular dynamic simulations on the dissociation of the adsorbed water and hydroxyl at the Re site on the Rh-Re surface in water.

The mean forces on the constraints or the average of the gradients of the free energy $<f>$ were calculated after each of the constrained AIMD simulations. The resulting mean forces along 
the reaction coordinate shown in Figure 5.5 indicate that the mean force becomes negative after gradually decreasing the value of $\xi$, and then goes back to zero when $\xi$ is about $-0.17 \AA$ thus indicating the transition state is close to the structure at $\xi=-0.17 \AA$. The mean force changes sign and becomes positive and finally goes back to zero as the value of $\xi$ is decreased further. It ultimately reaches the minimum which corresponds to the product state on the free energy surface.
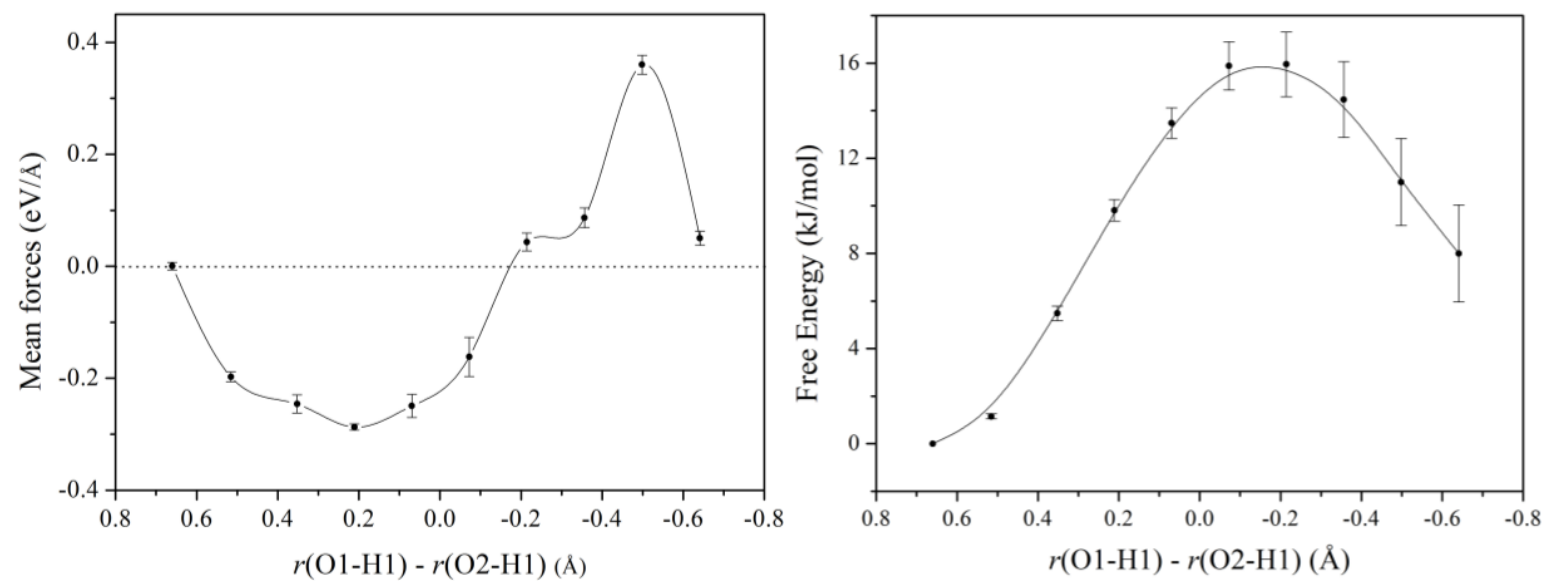

Figure 5.5 Mean forces on the constraints (left) and the free energy profiles (right) along the reaction coordinate calculated by the constrained AIMD simulations for the dissociation of adsorbed water on the Re site on the Rh-Re surface aqueous solution.

The free energy reaction profile for the dissociation of water at the Re site on the $\mathrm{Rh}-\mathrm{Re}$ alloy surface was calculated by thermodynamically integrating the mean forces along the reaction coordinate and the results were reported in Figure 5.5. The free energy profile provides for the clear location of the transition state with $\xi$ at about $-0.17 \AA$, which is slightly different from the transition state structure located by the static DFT calculations with $\xi$ at about $-0.12 \AA$. The free energy difference between the states at $\xi=-0.12 \AA$ to $\xi=-0.17 \AA$, however, is less than 1 $\mathrm{kJ} / \mathrm{mol}$. The free energy barrier and the overall free energy of reaction for the dissociation of 
water at Rh-Re alloy site were calculated to be $16 \pm 1 \mathrm{~kJ} / \mathrm{mol}$ and $8 \pm 2 \mathrm{~kJ} / \mathrm{mol}$, respectively. The constrained AIMD free energy barrier was slightly $(3 \mathrm{~kJ} / \mathrm{mol})$ higher than the activation energy calculated from the static DFT $(13 \mathrm{~kJ} / \mathrm{mol})$. The overall free energy change for the reaction, however was calculated to be $11 \mathrm{~kJ} / \mathrm{mol}$ more endothermic than reaction energies from static DFT calculations. The difference is likely due to entropy effects which are not included in the static DFT calculations that were carried out. Assuming that the activation barriers and the reaction energies for the dissociation of water calculated via the static DFT calculations are equal to the activation and overall reaction enthalpies, the activation entropy $\left(\triangle S_{A C T}\right)$ and the overall reaction entropy $\left(\Delta S_{r x n}\right)$ were calculated to be about $-10 \mathrm{~J} /(\mathrm{mol} \cdot \mathrm{K})$ and $-37 \mathrm{~J} /(\mathrm{mol} \cdot \mathrm{K})$, respectively. This suggests that there is a decrease in entropy in the transition state as well as the product state compared to that of the reactant state, which is reasonable considering that the dissociation of water forms the positive charged proton in the solution, which strongly interacts with the water molecules in solution and tends to order the water molecules in the solvent and thus decreases the entropy of the system. It's been reported that the entropy contribution is always unfavorable for the dissociation of homogenous acids. ${ }^{[156,157]}$

Similar constrained AIMD simulations were carried out to examine the dissociation of the adsorbed hydroxyl at the Re site of the Rh-Re alloy surface. The same constrained reaction coordinate $\xi=r(\mathrm{O} 1-\mathrm{H})-r(\mathrm{O} 2-\mathrm{H})$ was used in the AIMD simulations, which starts from $\xi=0.56$ $\AA$ followed by the fixed value of $\xi$ at $0.41 \AA, 0.27 \AA, 0.13 \AA,-0.01 \AA,-0.16 \AA,-0.30 \AA,-0.44 \AA$, $-0.58 \AA \mathrm{ad}-0.73 \AA$ along the reaction coordinate as is shown in Figure 5.3. The mean forces were calculated from the dynamics simulations at each point along the constrained trajectory and are shown in Figure 5.4. The free energy profile obtained from the thermodynamic integration of the mean forces along the reaction coordinate is also shown in Figure 5.4. The mean forces along 
the reaction coordinate and the free energy profile results indicate that the transition state for the dissociation of the hydroxyl at the Re site of the Rh-Re surface occurs at $\xi=0.11 \AA$, which is similar to the transition state structure located by the static DFT calculations $(\xi=0.12 \AA)$. The free energy barrier was calculated to be $12 \pm 2 \mathrm{~kJ} / \mathrm{mol}$ and the free energy change for the reaction was calculated to be $-13 \pm 4 \mathrm{~kJ} / \mathrm{mol}$.
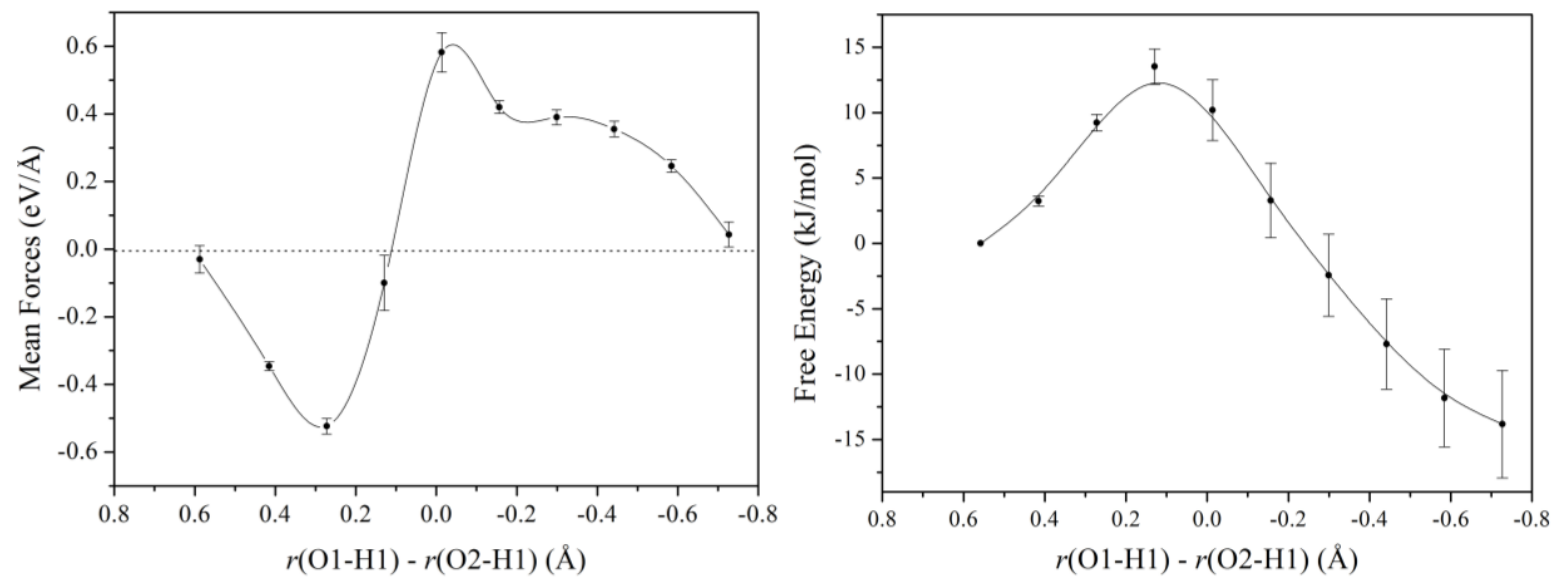

Figure 5.6 Mean forces on the constraints (left) and the free energy profiles (right) along the reaction coordinate calculated by the constrained AIMD simulations for the dissociation of adsorbed hydroxyl intermediates on the Re site on the Rh-Re surface in water.

Compared to the static DFT calculations, the free energy barrier was also found to be slightly $(2 \mathrm{~kJ} / \mathrm{mol}$ ) higher and the free energy change was $\sim 9 \mathrm{~kJ} / \mathrm{mol}$ more endothermic. These differences are likely due to the contributions from entropy which are not included in the static calculations which only consider changes in the potential energy. If we substitute the potential energy changes calculated by the static DFT calculations for the enthalpic changes, we can then calculate the entropy change associated with $\Delta S_{A C T}$ calculated to be about $-7 \mathrm{~J} /(\mathrm{mol} \cdot \mathrm{K})$ and $\Delta S_{r x n}$ is calculated to be about $-30 \mathrm{~J} /(\mathrm{mol} \cdot \mathrm{K})$. This decreased entropy for the dissociation of the adsorbed hydroxyl on the Rh-Re surface in water solution is also consistent with the dissociation 
of water we showed above and the reported dissociation of homogeneous acids in water. ${ }^{[156,157]}$

\subsubsection{Dissociation constants for the adsorbed water and hydroxyl on Rh-Re surface}

The free energy changes for the dissociation of the adsorbed water and hydroxyl intermediates adsorbed to the Rh-Re surface as Brønsted acids can be used to calculate the dissociation constant $K_{a}$ as well as the $p K_{a}$ for this reaction by Equations 5.9 and Equation 5.10, respectively. The dissociation constant for the adsorbed water on the Rh-Re surface was calculated here to be $4.0 \times 10^{-2}$ while the value of the $p K_{a}$ was calculated to be 1.39 in water at the temperature of $300 \mathrm{~K}$. The dissociation constant for the adsorbed hydroxyl on the Rh-Re surface was calculated to be $1.8 \times 10^{2}$ while the value of the $p K_{a}$ was calculated to be -2.26 in water at $300 \mathrm{~K}$.

$$
\begin{gathered}
K_{a}=\exp \left(-\frac{\Delta G}{R T}\right) \\
p K_{a}=\frac{\Delta G}{2.303 R T}
\end{gathered}
$$

The dissociation constant provides a direct measure of the strength of an acid in solution. The value of $p K_{a}$ for adsorbed water (1.39) is comparable to that for the homogeneous acid $\mathrm{H}_{3} \mathrm{PO}_{4}$ in water ${ }^{[158]}$ whereas the acidity of the adsorbed hydroxyl on the Rh-Re surface $(-2.26)$ is comparable to the homogeneous acid $\mathrm{HNO}_{3}$ in water. ${ }^{[159]}$

The negative free energy change and the low $p K_{a}$ calculated for the dissociation of the adsorbed hydroxyl at the $\mathrm{Re}$ sites on the $\mathrm{Rh}-\mathrm{Re}$ surface indicate that the corresponding $\mathrm{O} *$ surface species along with a hydronium ion in the solution phase is thermodynamically more favorable and as such adsorbed hydroxyl intermediates act as the Brønsted acid site on the Rh-Re catalysts tend to dissociate in water solution. 
It is important, however, to note that the calculations and results reported here were carried out with only one adsorbed hydroxyl on the Rh-Re alloy surface. If there is more than just one hydroxyl intermediate (acid site), which is the case for the actual Rh-Re catalyst, the dissociation of the first hydroxyl group will transfer an electron into the metal surface as have shown above which will increase the proton affinity of the metal surface thus making it more difficult to dissociate the second hydroxyl group just as that is found in multi-protic homogeneous acids as well as on supported heteropolyacids. ${ }^{[118,119]}$ Further removal of protons would continue to make it more difficult to undergo further deprotonation.

The heterolytic dissociation of the surface hydroxyl intermediate results in a proton in solution as well as an electron that is partially localize on the metal as well as on the oxygen. This is analogous to electrochemical systems in which the electrochemical potential sets the oxidation or reduction of oxygen-surface intermediates. ${ }^{[160]}$ In our hydrogenolysis system the "potential" is not set by an external potential but instead dictated by the equilibrium between the gas phase hydrogen and the formation of the solution phase hydronium ion and the negatively charged metal surface via the equation:

$$
0.5 \mathrm{H}_{2} \rightarrow \mathrm{H}^{+}+e^{-}
$$

The high pressure $\mathrm{H}_{2}$ that is used will therefore result in a more negatively charged (reduced) surface which will make it more difficult to activate the surface hydroxyl intermediates on the Rh-Re metal surface. The equilibrium between the adsorbed hydroxyl species and the gas phase $\mathrm{H}_{2}$ at the reaction condition examined herein indicate that the Re sites on the Rh-Re surface are predominantly covered by the hydroxyl intermediates with very low concentrations of surface oxygen. The investigation of the dissociation of adsorbed water and hydroxyl on the Rh-Re surface with potentials on the metal surface requires the use of the double reference method ${ }^{[160]}$ 
developed to analyze electrochemical systems and is proposed as future work.

\subsubsection{Entropy changes for the Ring opening of THFA at the acid sites on Rh-Re surface}

The ring opening of the cyclic ether THFA at the Re-OH acid site on the Rh-Re surface in aqueous solution was calculated by static DFT calculations and discussed previously in Chapter 3. The reaction was found to proceed via a concerted protonation, ring opening and $\mathrm{H}$ transfer process. The aqueous media was found to stabilize the transition state. While these calculations provide the changes in potential energy, they neglect the changes in entropy which may be important for such ring opening reactions. They also did not consider the dynamic nature of water and the number of different possible configurations of water. Herein, we carry out the constrained ab initio molecular dynamics (AIMD) simulation to follow the entropy and free energy changes during the course of the reaction. As shown in Figure 5.7, six constrained reaction coordinates are required to follow the ring opening reaction of THFA at the Re-OH acid site on the Rh-Re surface with constrained AIMD simulations: $\xi_{1}=d(\mathrm{O} 1-\mathrm{H} 1)-d(\mathrm{O} 2-\mathrm{H} 1), \xi_{2}=$ $d(\mathrm{C} 2-\mathrm{O} 2), \quad \xi_{3}=d(\mathrm{C} 1-\mathrm{H} 2)-d(\mathrm{O} 3-\mathrm{H} 2), \xi_{4}=d(\mathrm{C} 2-\mathrm{H} 2), \xi_{5}=d(\mathrm{O} 4-\mathrm{H} 3)-d(\mathrm{O} 5-\mathrm{H} 3)$ and $\xi_{6}=d(\mathrm{O} 2-$ $\mathrm{Rh})$.

The structure for the free energy transition state is required to calculate the free energy barrier for the ring opening of THFA at the Re-OH acid site on the Rh-Re surface by the constrained molecular dynamics simulation. The algorithms ${ }^{[161]}$ needed to locate the free energy

transition state have not been implemented yet in the VASP code. We therefore use here instead the potential energy transition state structure located by the static DFT calculations to approximate the free energy transition state structure. It's been reported that the free energy transition state structure is very close to the potential energy transition state at the modest 
temperature of $300 \mathrm{~K}$ for the proton exchange reaction of hydrocarbons over acidic zeolite. ${ }^{[150]}$

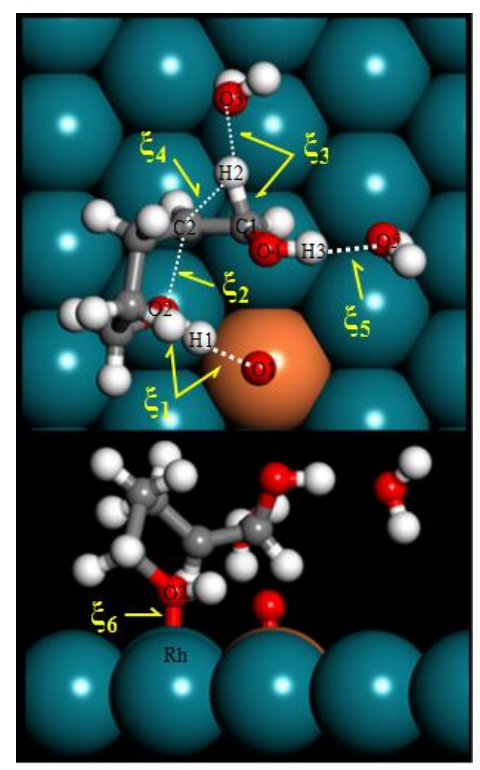

Figure 5.7 Constrained reaction coordinates for the constrained molecular dynamics simulations on the ring opening of THFA at the Re-OH acid site on the Rh-Re surface in water.

The constrained molecular dynamics simulations for the ring opening of THFA at the Re$\mathrm{OH}$ acid site on the Rh-Re surface were carried out by fixing the set of $\left[\xi_{1}, \xi_{2}, \xi_{3}, \xi_{4}, \xi_{5}, \xi_{6}\right]$ in different values along the reaction coordinate as shown in Table 5.3. After the molecular dynamics simulations were finished, the mean forces on the constraints were calculated as also shown in Table 5.3. We can see that the mean forces on the six constrained reaction coordinates of the transition state structure are less than $0.1 \mathrm{eV} / \AA$, which are small enough compared to the system error of the constrained molecular dynamics methods, indicating the transition state structure located by the static DFT calculations is similar to the free energy transition state structure. 
Table 5.3 Fixed values of the set of constrained reaction coordinates and the calculated mean forces on the constraints calculated by the constrained molecular dynamics simulations on the ring opening of THFA at the Re-OH acid site on the Rh-Re surface in water. The units for the constrained reaction coordinates $\xi$ are in $\AA$, and the units for the mean forces are in $\mathrm{eV} / \AA$.

\begin{tabular}{|c|c|c|c|c|c|c|c|c|c|c|c|c|}
\hline & $\xi_{1}$ & $\langle f\rangle_{\xi 1}$ & $\xi_{2}$ & $\langle f\rangle_{\xi 2}$ & $\xi_{3}$ & $\langle f\rangle_{\xi 3}$ & $\xi_{4}$ & $\langle f\rangle_{\xi 4}$ & $\xi_{5}$ & $\langle f\rangle_{55}$ & $\xi_{6}$ & $\langle f\rangle_{\xi 6}$ \\
\hline R & 0.71 & -0.08 & 1.48 & -0.00 & 1.68 & -0.04 & 2.11 & -0.09 & 0.85 & 0.06 & 2.66 & -0.02 \\
& & & & & & & & & & & & \\
\hline IMG1 & 0.57 & -0.20 & 1.58 & 1.06 & 1.57 & -0.10 & 2.03 & -0.36 & 0.81 & 0.18 & 2.61 & -0.12 \\
\hline IMG2 & 0.44 & -0.34 & 1.67 & 1.44 & 1.47 & -0.02 & 1.95 & -0.58 & 0.78 & 0.13 & 2.57 & -0.06 \\
\hline IMG3 & 0.30 & -0.42 & 1.77 & 1.36 & 1.36 & -0.08 & 1.87 & -0.82 & 0.74 & 0.03 & 2.53 & 0.01 \\
\hline IMG4 & 0.17 & -0.39 & 1.86 & 1.23 & 1.25 & -0.08 & 1.79 & -0.89 & 0.70 & -0.02 & 2.49 & -0.01 \\
\hline IMG5 & 0.03 & -0.24 & 1.96 & 0.88 & 1.13 & -0.15 & 1.72 & -0.64 & 0.66 & -0.03 & 2.44 & 0.14 \\
\hline IMG6 & -0.10 & 0.07 & 2.05 & 0.41 & 1.02 & 0.09 & 1.64 & -0.43 & 0.62 & 0.02 & 2.40 & 0.11 \\
\hline IMG7 & -0.24 & 0.16 & 2.15 & 0.22 & 0.91 & -0.02 & 1.57 & -0.31 & 0.58 & -0.04 & 2.36 & 0.05 \\
\hline IMG8 & -0.37 & 0.18 & 2.25 & 0.10 & 0.80 & 0.09 & 1.50 & -0.15 & 0.54 & 0.02 & 2.32 & 0.02 \\
\hline TST & -0.50 & 0.06 & 2.34 & 0.02 & 0.69 & 0.06 & 1.43 & 0.08 & 0.50 & 0.01 & 2.28 & 0.04 \\
\hline
\end{tabular}

The free energy profile from the reactant state to the transition state for the ring opening of THFA at the Re-OH acid site on the Rh-Re surface was then calculated by the thermodynamic integration of the mean forces along the reaction coordinates as shown in Figure 5.7. The free energy barrier is calculated to be $111 \pm 6 \mathrm{~kJ} / \mathrm{mol}$, which is significant higher than that for the static DFT calculations $(84 \mathrm{~kJ} / \mathrm{mol})$. If using the static DFT calculated potential energy barrier to approximate the enthalpy change, the entropy contribution to the activation barrier is calculated to be about $-90 \mathrm{~J} /(\mathrm{mol} \cdot \mathrm{K})$. The decreased entropy from the reactant to the transition state is due to the stronger interaction between the solvent water and the charged transition state as have been shown in Chapter 3. Thus the solvent water can affect the ring opening reaction of THFA at the Re-OH acid site on the Rh-Re surface in both enthalpy change and entropy change. 


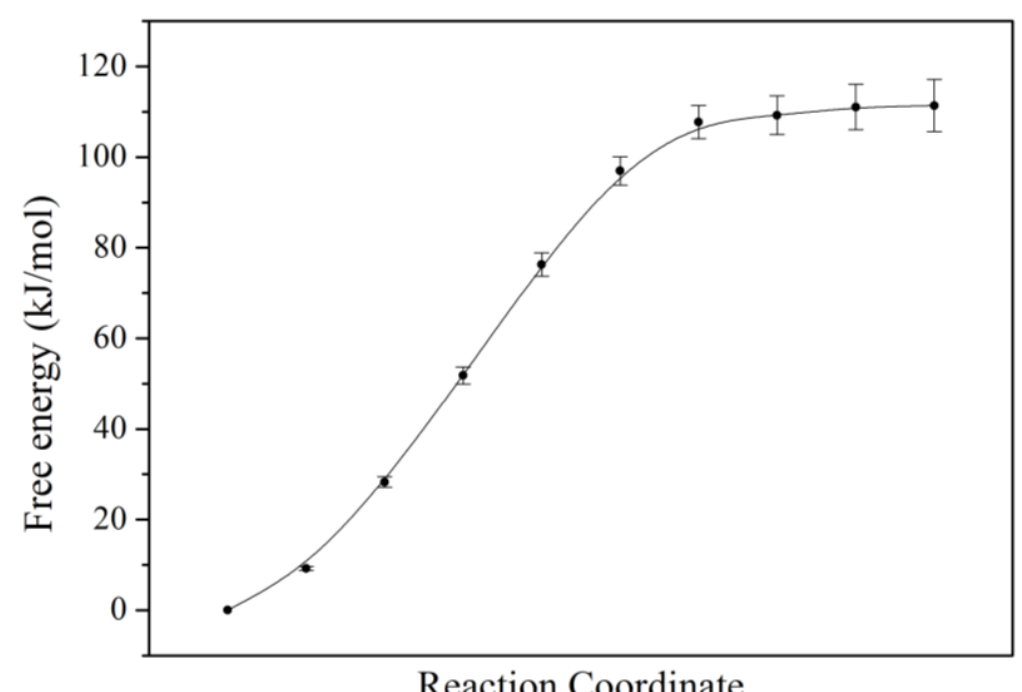

Figure 5.8 Free energy profiles calculated by the constrained molecular dynamics and thermodynamic integration for the ring opening of THFA at the Re-OH acid site on the Rh-Re surface in water.

The static DFT calculations showed that without the solvent water, the activation barrier for the ring opening of THFA at the Re-OH acid site on the Rh-Re surface was calculated to be 160 $\mathrm{kJ} / \mathrm{mol}$. Thus the presence of solvent water can lower the potential energy barrier by about 76 $\mathrm{kJ} / \mathrm{mol}$ due to the stabilization of the transition state by water. The strong stabilization of the transition state by water in turn however will also decrease the entropy of the system, which contributes about $27 \mathrm{~kJ} / \mathrm{mol}$ to the barrier for the ring opening of THFA at the temperature of 300 $\mathrm{K}$. The potential energy contribution to the activation barrier due to the presence of the solvent water is more significant than the entropy contribution, thus the total effect of the solvent water is to facilitate the ring opening reaction of THFA at the Re-OH acid site on the Rh-Re surface.

Considering the entropy changes in the ring opening of THFA at the Re-OH acid site on the $\mathrm{Rh}-\mathrm{Re}$ surface, the free energy barrier is about $27 \mathrm{~kJ} /$ higher than the potential energy barrier we showed in Chapter 3, which changes the energy diagram we showed in Figure 3.8 for the overall hydrogenolysis diagram for THFA hydrogenolysis on the Rh-Re surface including the 
hydrogenation steps on the metal sites and changes our understanding of the kinetics. However, as also shown in Chapter 3, the metal atoms around the acid sites and the acidity of the acid sites can both influence the potential energy barriers for the ring opening reaction. Thus, even with entropy changes considered for the ring opening step of THFA, the rate limiting step at the second hydrogenation step we proposed previously may still not change if the ring opening reaction occurs at the edge or corner acid sites or at the acid sites with stronger acidity on the RhRe catalysts.

\subsection{Conclusions}

In this chapter, we studied the heterolytic dissociation of the adsorbed water at the Re sites on the $\mathrm{Rh}-\mathrm{Re}$ surface to form a locally solvated hydronium ion near the metal and hydroxyl intermediates on the metal surface. The reaction proceeds via proton-coupled electron transfer reaction where the proton goes on to form a hydronium ion stabilized by water molecules at the interface, a negative charge located on both the metal surface and the surface $\mathrm{OH}^{*}$ intermediate. The activation barrier for the heterolytic dissociation of water was calculated to be $13 \mathrm{~kJ} / \mathrm{mol}$, which is much lower than the metal insertion route that proceeds for the gas phase activation of water over a metal surface. Constrained ab initio molecular dynamics simulation showed that the free energy for the dissociation of water at the Re site on the Rh-Re alloy was less favorable than that for the enthalpy due to entropic losses that result in the strong interactions between local water molecules and the charged hydronium ion that forms, which is in consistent with the reported dissociation of homogeneous acids in water.

The activation barrier for the dissociation of the adsorbed hydroxyl that result at the Re site on the $\mathrm{Rh}$-Re surface to form the hydronium ions in water solution and oxygen intermediates on 
the metal surface was found to be lower than that for dissociation of the adsorbed water at these same sites. In addition, the reaction energy for the dissociation of the $\mathrm{O}-\mathrm{H}$ bond for $\mathrm{Re}-\mathrm{OH}$ bond was found to be more exothermic than the $\mathrm{O}-\mathrm{H}$ bond for the dissociation of $\mathrm{Re}-\mathrm{OH}_{2}$. These results are consistent with the trend of the acidities of the adsorbed water and hydroxyl at the terrace $\mathrm{Re}$ atom of the $\mathrm{Rh}-\mathrm{Re}$ particle we showed previously. Constrained ab initio molecular dynamics simulation also showed a loss of entropy for the dissociation of the adsorbed hydroxyl, which is consistent with the dissociation of the adsorbed water on the Rh-Re surface and the dissociation of homogeneous acids.

The negative free energy change for the dissociation of the adsorbed hydroxyl on the Rh-Re surface and the low $p K_{a}$ indicates the adsorbed hydroxyl on the $\mathrm{Rh}$-Re surface tends to dissociate and the hydronium ions in the water solution is thermodynamically more favorable. However, if there are more hydroxyl groups on the Rh-Re surface, the continued dissociation of the second hydroxyl will be more difficult than the first hydroxyl due to the extra charge on the metal surface formed by the dissociation of the first hydroxyl.

Constrained $\mathrm{ab}$ initio molecular dynamics simulations for the ring opening of THFA at the Re-OH acid site on the Rh-Re surface revealed a free energy barrier of $\sim 111 \mathrm{~kJ} / \mathrm{mol}$, which indicates the entropy contribution to the energy barrier is significant at about $27 \mathrm{~kJ} / \mathrm{mol}$ at the temperature of $300 \mathrm{~K}$. The loss in entropy in going from the reactant state to the transition state is due to the stronger interaction of the solvent water with the charged transition state structure. The solvent water molecules were found to lower the enthalpy and decrease the entropy for the ring opening of THFA. The solvent water can stabilize the transition state, which decreases the activation barrier by about $76 \mathrm{~kJ} / \mathrm{mol}$. The stabilization of the transition state by the solvent water however in turn results in a loss in entropy. The increased stabilization due to enthalpy wins out 
over the loss in entropy due to the stabilization of the transition state, thus resulting in an overall stabilization of the transition state and overall lower reaction energy involved in the ring opening of THFA at the Re-OH acid site on the Rh-Re surface. 


\section{Chapter 6-Summary and Conclusions}

First-principle quantum chemical calculations were used along with detailed kinetic analyses in this dissertation to understand the fundamental mechanisms that control the selective hydrogenolysis of biomass-derived cyclic ethers and polyols on non-promoted and Re promoted $\mathrm{Rh}$ catalyst. We examined the reaction paths for the hydrogenolysis of the simple model polyol compound 1,2-propanediol on non-promoted $\mathrm{Rh}$ surface. The results showed that the $\mathrm{C}-\mathrm{O}$ bond breaking of 1,2-propanediol at the less substituted terminal carbon to form 2-propanol proceeds through the formation of the $\mathrm{CH}_{3} \mathrm{CH}(\mathrm{OH}) \mathrm{CH}^{*} \mathrm{OH}$ hydroxyalkyl intermediate which is formed by activating the terminal $\mathrm{C}-\mathrm{H}$ bond. Similarly, the C-O breaking of 1,2-propanediol at the more substituted center carbon to form 1-propanol proceeds through a similar $\mathrm{CH}_{3} \mathrm{C}^{*}(\mathrm{OH})-\mathrm{CH}_{2} \mathrm{OH}$ hydroxyl alkyl intermediate which, however, is not formed by directly activating the central C-H bond at the center carbon of 1,2-PDO, but is formed instead through multiple steps involving the hydrogenation of the ketone intermediate $\mathrm{CH}_{3}\left(\mathrm{C}^{*}=\mathrm{O}^{*}\right)-\mathrm{CH}_{2} \mathrm{OH}$. Kinetics analyses of the hydrogenolysis of 1,2-propanol on the $\mathrm{Rh}(111)$ surface shows that $\mathrm{OH}^{*}$ and $\mathrm{CH}_{3} \mathrm{CH}(\mathrm{OH}) \mathrm{CH} * \mathrm{OH}$ may build up the metal surface and the hydrogenation of $\mathrm{OH}^{*}$ and the $\mathrm{C}-\mathrm{O}$ activation of $\mathrm{CH}_{3} \mathrm{CH}(\mathrm{OH}) \mathrm{CH}^{*} \mathrm{OH}$ steps are likely to be the rate limiting steps at low hydrogen pressure. It's also found that selectivity of 2-propanol is higher than that over 1-propanol, indicating the less substituted terminal $\mathrm{C}-\mathrm{OH}$ is more reactive in the hydrogenolysis of 1,2-PDO on $\mathrm{Rh}(111)$ surface. Comparison of the hydrogenolysis of 1,2-propanediol with the monofunctional alcohol 1-propanol and 2-propanol showed that the $\alpha-\mathrm{OH}$ in the molecule of 1,2propanol can facilitate the $\mathrm{O}-\mathrm{H}$ bond, $\mathrm{C}-\mathrm{H}$ bond and $\mathrm{C}-\mathrm{O}$ bond activation steps that are important in the hydrogenolysis process on the $\mathrm{Rh}(111)$ surface.

We subsequently examined the selective hydrogenolysis of tetrahydrofurfural alcohol 
(THFA) and other cyclic ethers on the Re-promoted Rh catalyst. The selectivity of the hydrogenolysis of THFA on the Rh-Re catalyst does not appear to proceed via the metalcatalyzed mechanism. The reaction instead appears to proceed via acid sites on the Re-promoted $\mathrm{Rh}$ catalyst which were further confirmed by both the experiments and our DFT calculations. The acid catalyzed ring opening of THFA proceeds at Re-OH sites via a proton-coupled electron transfer path involving a concerted protonation, ring opening and $\mathrm{H}$ transfer process to form an oxocarbenium ion intermediate. Both the presence of the $\mathrm{OH}$ group that is $\alpha$ to the $\mathrm{C}-\mathrm{O}$ bond activated and protic solvents such as water can help to stabilize the transition state and lower the activation barrier. In aqueous solution, the oxocarbenium ion is not stable and deprotonates to the water solvent to form the $\delta$-hydroxyvaleraldehyde intermediate. The proton in water then shuttles back to regenerate the surface acid sites and the formed $\delta$-hydroxyvaleraldehyde intermediate then hydrogenates through an hydroxylalkyl intermediate at the $\mathrm{Rh}$ metal sites to form the final product 1,5-pentanediol.

The DFT calculations combined with the experiments showed that the second hydrogenation step of $\delta$-hydroxyvaleraldehyde at the metal site is likely to be rate-limiting in the hydrogenolysis of THFA on the Rh-Re catalyst. The proposed elementary steps were used to establish a detailed rate expression for the hydrogenolysis kinetics for THFA over Rh-Re alloy which reveals a first order dependence on the hydrogen partial pressure as well as a first order dependence on the concentration of THFA at the low THFA concentration conditions which agrees with the results from experiments.

Ring opening of THFA at different acid sites on Rh-Re surfaces showed that the acidity of the Re site as well as the degree of coordination of the vicinal Rh sites influence the activation barriers. The acid site strength enhances the rate of proton transfer and rate of ring opening 
whereas the coordinatively-unsaturated $\mathrm{Rh}$ sites stabilize the $\mathrm{OH}$ group formed in the transition state which lowers the activation barrier for the ring opening of THFA. It was also found that the adsorption energy of THFA and the activation barrier for ring opening at different acid sites on the Rh-Re surface can both linearly correlated to the deprotonation energy of the acid sites. These correlations also appear to hold for the ring opening of THFA at the acid sites on a series of bimetal alloys comprised of other reducible metals such as Rh, Pt, Ir and Pd and other oxophilic promoter metals such as Re, Mo, $\mathrm{W}$ and $\mathrm{Ru}$.

The mechanism for the hydrogenolysis of THFA over the Rh-Re catalyst was extended to other cyclic ethers and polyols through the use of Born-Haber cycle analysis. The activation barriers for the $\mathrm{C}-\mathrm{O}$ bond activation of various cyclic ethers and polyols at the Rh-Re acid site were found to be linearly correlated to their gas phase carbenium ion formation energies.

In order to provide further insights into the nature of active Re sites we examined adsorbed water $\mathrm{Re}-\mathrm{OH}_{2}$, hydroxyl intermediates $\mathrm{Re}-\mathrm{OH}$ and the unoccupied metallic Re sites in aqueous system based on the experimental characterization results reported in the literature. Both the Re$\mathrm{OH}_{2}$ and Re-OH sites appear to behave as Brønsted acid sites. $\mathrm{Re}-\mathrm{OH}_{2}$ sites at the edge and corners of the cluster were found to be even more acidic than their corresponding Re-OH sites. In addition to the Brønsted acid sites, the unoccupied Re sites on the Rh-Re surface were found to act as Lewis acid sites.

Detailed charge analyses showed that even the metallic Re atom sites without adsorbed $\mathrm{H}_{2} \mathrm{O}$ or $\mathrm{OH}$ result in positively charged Re centers as a result of the charge transfer to the neighboring Rh sites. The adsorption of $\mathrm{H}_{2} \mathrm{O}$ or $\mathrm{OH}$ at the Re sites increase the positive charge on the Re atoms. 
The ring opening of THFA at the Re- $\mathrm{OH}_{2}$ sites showed the similar transition state as at the Re-OH acid site with even lower energy barriers at the edge and corner $\mathrm{Re}-\mathrm{OH}_{2}$ sites due to the stronger acidity. The ring opening of THFA at the unoccupied Re sites on the Rh-Re surface proceeds via the Lewis acid catalyzed concerted ring opening and $\mathrm{H}$ transfer mechanism similar to that at the Brønsted acid site $\mathrm{Re}-\mathrm{OH}$ and $\mathrm{Re}-\mathrm{OH}_{2}$. The charge analysis of the transition state showed the negative charged center on the Rh-Re metal surface and the positive charge center on the carbenium ion in the transition state, which is similar to the transition states reported for typical Lewis acid catalyzed ring opening of epoxides, confirmed the ring opening of THFA at the unoccupied metallic Re site on the Rh-Re surface is likely to be a Lewis acid catalyzed chemistry. Both the adsorption energies of THFA and the activation barriers for its ring opening at different unoccupied Re sites on the Rh-Re particle were found to be linearly correlated to the binding of $\mathrm{NH}_{3}$ at these different sites, which is a probe of Lewis acid acidity.

Even though the Re-OH, Re-OH 2 and the unoccupied Re sites on the Rh-Re surface can all be possible acid sites that catalyze the ring opening of THFA and other cyclic ethers and polyols, the analyses of the coverage of these different sites showed Re-OH is the dominant sites on the $\mathrm{Rh}-\mathrm{Re}$ surface at the reaction condition, which suggests the $\mathrm{Re}-\mathrm{OH}$ is more likely to be the acid site on the Rh-Re catalyst that is responsible for the ring opening of THFA and other cyclic ethers and polyols.

In water solutions, Brønsted acid sites are likely to dissociate to form the hydronium ions in the solution, which will influence the reactions that occur at the surface acid sites. The activation of water at Re site of the Rh-Re alloy surface proceeds through the heterolytic path to form a local solvated hydronium intermediate near the metal surface and Re-OH species. The activation of the $\mathrm{O}-\mathrm{H}$ bonds from the $\mathrm{Re}-\mathrm{OH}$ and $\mathrm{Re}-\mathrm{OH}_{2}$ sites were examined in detail using constrained 
ab initio molecular dynamics simulations to establish the free energy changes for reaction. The results confirmed that dissociation proceeds heterolytically at the Re center. The free energy barrier and free energy change for the dissociation of the adsorbed hydroxyl Re-OH on the RhRe surface to form the hydronium ion is about $12 \mathrm{~kJ} / \mathrm{mol}$ and $-13 \mathrm{~kJ} / \mathrm{mol}$ respectively. The dissociation of $\mathrm{Re}-\mathrm{OH}_{2}$ and $\mathrm{Re}-\mathrm{OH}$ both resulted in entropic penalties which is consistent with the reported dissociation of homogenous acids. The negative free energy change for the dissociation of $\mathrm{Re}-\mathrm{OH}$ on the $\mathrm{Rh}-\mathrm{Re}$ surface and the calculated low $p K_{a}$ indicates the $\mathrm{ReOH}$ site on the Rh-Re surface dissociates and the hydronium ion in the water solution is thermodynamically more favorable. However, if there're more hydroxyl groups on the Rh-Re surface, the subsequent dissociation of the second hydroxyl is much more difficult than the first hydroxyl as the negative charge on the metal that results significantly decreases the acidity of the metal which is analogous to what occurs in multi-protic homogeneous acids.

Constrained molecular dynamics simulations on the ring opening of THFA at the Re-OH acids on the Rh-Re surface in water were carried out to understand the entropy effects. The results showed a decrease in entropy in moving from the reactant to the transition state due to the stronger interaction of the solvent water with the charged transition state. The loss of entropy results in a higher free energy barrier for the ring opening of THFA. The water solvent can therefore act to influence both the enthalpy and the entropy for the activation and ring opening of THFA at the Re-OH site. The solvent water can effectively stabilize the ring opening transition state, which lowers the activation energy by $\sim 76 \mathrm{~kJ} / \mathrm{mol}$. The stabilization of the transition state by the solvent water however leads to a loss in entropy. The enthalpy effect by the solvent water is more significant than the entropy change, which leads to the total effect of the solvent water is to facilitate the ring opening reaction of THFA at the $\mathrm{Re}-\mathrm{OH}$ acid site on the $\mathrm{Rh}-\mathrm{Re}$ surface. 


\section{References}

[1] T. Werpy, G. Petersen, Top Value Added Chemicals from Biomass: Volume 1-Results of Screening for Potential Candidates from Sugars and Synthesis Gas, U.S. Department of Energy (DOE), (2004).

[2] M. Chia, T.J. Schwartz, B.H. Shanks, J.A. Dumesic, Triacetic acid lactone as a potential biorenewable platform chemical, Green Chem., 14 (2012) 1850-1853.

[3] M. Chia, M.A. Haider, G. Pollock, G.A. Kraus, M. Neurock, J.A. Dumesic, Mechanistic Insights into RingOpening and Decarboxylation of 2-Pyrones in Liquid Water and Tetrahydrofuran, J. Am. Chem. Soc., 135 (2013) 5699-5708.

[4] G.W. Huber, J.N. Chheda, C.J. Barrett, J.A. Dumesic, Production of Liquid Alkanes by Aqueous-Phase Processing of Biomass-Derived Carbohydrates, Science (Washington, DC, U. S.), 308 (2005) 1446-1450.

[5] J.N. Chheda, J.A. Dumesic, An overview of dehydration, aldol-condensation and hydrogenation processes for production of liquid alkanes from biomass-derived carbohydrates, Catal. Today, 123 (2007) 59-70.

[6] J.N. Chheda, G.W. Huber, J.A. Dumesic, Liquid-phase catalytic processing of biomass-derived oxygenated hydrocarbons to fuels and chemicals, Angew. Chem., Int. Ed., 46 (2007) 7164-7183.

[7] J.N. Chheda, Y. Roman-Leshkov, J.A. Dumesic, Production of 5-hydroxymethylfurfural and furfural by dehydration of biomass-derived mono- and poly-saccharides, Green Chem., 9 (2007) 342-350.

[8] Y. Roman-Leshkov, C.J. Barrett, Z.Y. Liu, J.A. Dumesic, Production of dimethylfuran for liquid fuels from biomass-derived carbohydrates, Nature (London, U. K.), 447 (2007) 982-985.

[9] E.L. Kunkes, D.A. Simonetti, R.M. West, J.C. Serrano-Ruiz, C.A. Gaertner, J.A. Dumesic, Catalytic conversion of biomass to monofunctional hydrocarbons and targeted liquid-fuel classes, Science (Washington, DC, U. S.), 322 (2008) 417-421.

[10] R.M. West, Z.Y. Liu, M. Peter, J.A. Dumesic, Liquid alkanes with targeted molecular weights from biomassderived carbohydrates, ChemSusChem, 1 (2008) 417-424.

[11] D.A. Simonetti, E.L. Kunkes, R.M. West, J.C. Serrano-Ruiz, C.A. Gartner, J.A. Dumesic, Production of monofunctional hydrocarbons from biomass derived carbohydrates viz catalytic conversion on carbon supported platinum-rhenium, Prepr. - Am. Chem. Soc., Div. Pet. Chem., 54 (2009) 35-38.

[12] R.M. West, E.L. Kunkes, D.A. Simonetti, J.A. Dumesic, Catalytic conversion of biomass-derived carbohydrates to fuels and chemicals by formation and upgrading of mono-functional hydrocarbon intermediates, Catal. Today, 147 (2009) 115-125.

[13] R.M. West, M.H. Tucker, D.J. Braden, J.A. Dumesic, Production of alkanes from biomass derived carbohydrates on bi-functional catalysts employing niobium-based supports, Catal. Commun., 10 (2009) 1743-1746.

[14] D.M. Alonso, J.Q. Bond, J.A. Dumesic, Catalytic conversion of biomass to biofuels, Green Chem., 12 (2010) 1493-1513.

[15] J.C. Serrano-Ruiz, D.J. Braden, R.M. West, J.A. Dumesic, Conversion of cellulose to hydrocarbon fuels by progressive removal of oxygen, Appl. Catal., B, 100 (2010) 184-189.

[16] J.C. Serrano-Ruiz, R.M. West, J.A. Dumesic, Catalytic conversion of renewable biomass resources to fuels and chemicals, Annu. Rev. Chem. Biomol. Eng., 1 (2010) 79-100.

[17] J.C. Serrano-Ruiz, J.A. Dumesic, Catalytic routes for the conversion of biomass into liquid hydrocarbon transportation fuels, Energy Environ. Sci., 4 (2011) 83-99.

[18] D.M. Alonso, S.G. Wettstein, J.A. Dumesic, Bimetallic catalysts for upgrading of biomass to fuels and chemicals, Chem. Soc. Rev., 41 (2012) 8075-8098.

[19] S. Murat Sen, C.A. Henao, D.J. Braden, J.A. Dumesic, C.T. Maravelias, Catalytic conversion of lignocellulosic biomass to fuels: Process development and technoeconomic evaluation, Chem. Eng. Sci., 67 (2012) 57-67.

[20] S.G. Wettstein, D.M. Alonso, E.I. Gurbuz, J.A. Dumesic, A roadmap for conversion of lignocellulosic biomass to chemicals and fuels, Curr. Opin. Chem. Eng., 1 (2012) 218-224.

[21] C.-J. Yue, L.-P. Gu, Y. Su, S.-P. Zhu, Selective hydrogenolysis of glycerol to 1,2-propanediol over MgO-nested Raney Cu, React. Kinet., Mech. Catal., 111 (2014) 633-645.

[22] E.S. Vasiliadou, T.M. Eggenhuisen, P. Munnik, P.E. de Jongh, K.P. de Jong, A.A. Lemonidou, Synthesis and performance of highly dispersed $\mathrm{Cu} / \mathrm{SiO} 2$ catalysts for the hydrogenolysis of glycerol, Appl. Catal., B, 145 (2014) 108-119.

[23] S. Zhu, X. Gao, Y. Zhu, Y. Zhu, H. Zheng, Y. Li, Promoting effect of boron oxide on $\mathrm{Cu} / \mathrm{SiO} 2$ catalyst for glycerol hydrogenolysis to 1,2-propanediol, J. Catal., 303 (2013) 70-79.

[24] Z. Wu, Y. Mao, M. Song, X. Yin, M. Zhang, Cu/boehmite: A highly active catalyst for hydrogenolysis of glycerol to 1,2-propanediol, Catal. Commun., 32 (2013) 52-57. 
[25] D. Duran-Martin, M. Ojeda, M.L. Granados, J.L.G. Fierro, R. Mariscal, Stability and regeneration of Cu-ZrO2 catalysts used in glycerol hydrogenolysis to 1,2-propanediol, Catal. Today, 210 (2013) 98-105.

[26] F. Auneau, C. Michel, F. Delbecq, C. Pinel, P. Sautet, Unravelling the Mechanism of Glycerol Hydrogenolysis over Rhodium Catalyst through Combined Experimental-Theoretical Investigations, Chem. - Eur. J., 17 (2011) 14288-14299, S14288/14281-S14288/14288.

[27] M. Checa, F. Auneau, J. Hidalgo-Carrillo, A. Marinas, J.M. Marinas, C. Pinel, F.J. Urbano, Catalytic transformation of glycerol on several metal systems supported on ZnO, Catal. Today, 196 (2012) 91-100.

[28] A. Wolosiak-Hnat, E. Milchert, G. Lewandowski, S. Lenart, Activity of selected catalysts in hydrogenolysis of glycerol to 1,2- and 1,3-propanediol, Oxid. Commun., 35 (2012) 77-91.

[29] S. Wang, K. Yin, Y. Zhang, H. Liu, Glycerol Hydrogenolysis to Propylene Glycol and Ethylene Glycol on Zirconia Supported Noble Metal Catalysts, ACS Catal., 3 (2013) 2112-2121.

[30] V. Pavankumar, C.S. Srikanth, A.N. Rao, K.V.R. Chary, Vapour phase hydrogenolysis of glycerol over nano $\mathrm{Ru} / \mathrm{SBA}-15$ catalysts on the effect of preparatory routes and metal precursors, J. Nanosci. Nanotechnol., 14 (2014) 3137-3146.

[31] Y. Li, L. Ma, H. Liu, D. He, Influence of HZSM5 on the activity of Ru catalysts and product selectivity during the hydrogenolysis of glycerol, Appl. Catal., A, 469 (2014) 45-51.

[32] J. Feng, W. Xiong, B. Xu, W. Jiang, J. Wang, H. Chen, Basic oxide-supported Ru catalysts for liquid phase glycerol hydrogenolysis in an additive-free system, Catal. Commun., 46 (2014) 98-102.

[33] B. Li, J. Wang, Y. Yuan, H. Ariga, S. Takakusagi, K. Asakura, Carbon Nanotube-Supported RuFe Bimetallic Nanoparticles as Efficient and Robust Catalysts for Aqueous-Phase Selective Hydrogenolysis of Glycerol to Glycols, ACS Catal., 1 (2011) 1521-1528.

[34] S.-H. Lee, D.J. Moon, Studies on the conversion of glycerol to 1,2-propanediol over Ru-based catalyst under mild conditions, Catal. Today, 174 (2011) 10-16.

[35] L. Ma, D. He, Hydrogenolysis of Glycerol to Propanediols Over Highly Active Ru-Re Bimetallic Catalysts, Top. Catal., 52 (2009) 834-844.

[36] T. Jiang, Y. Zhou, S. Liang, H. Liu, B. Han, Hydrogenolysis of glycerol catalyzed by Ru-Cu bimetallic catalysts supported on clay with the aid of ionic liquids, Green Chem., 11 (2009) 1000-1006.

[37] M. Balaraju, V. Rekha, P.S.S. Prasad, B.L.A.P. Devi, R.B.N. Prasad, N. Lingaiah, Influence of solid acids as cocatalysts on glycerol hydrogenolysis to propylene glycol over Ru/C catalysts, Appl. Catal., A, 354 (2009) 82-87.

[38] E.P. Maris, W.C. Ketchie, M. Murayama, R.J. Davis, Glycerol hydrogenolysis on carbon-supported PtRu and AuRu bimetallic catalysts, J. Catal., 251 (2007) 281-294.

[39] E.P. Maris, R.J. Davis, Hydrogenolysis of glycerol over carbon-supported Ru and Pt catalysts, J. Catal., 249 (2007) 328-337.

[40] J. Feng, J. Wang, Y. Zhou, H. Fu, H. Chen, X. Li, Effect of base additives on the selective hydrogenolysis of glycerol over Ru/TiO2 catalyst, Chem. Lett., 36 (2007) 1274-1275.

[41] D.G. Lahr, B.H. Shanks, Effect of sulfur and temperature on ruthenium-catalyzed glycerol hydrogenolysis to glycols, J. Catal., 232 (2005) 386-394.

[42] S.N. Delgado, L. Vivier, C. Especel, Polyol hydrogenolysis on supported Pt catalysts: Comparison between glycerol and 1,2-propanediol, Catal. Commun., 43 (2014) 107-111.

[43] S. Zhu, Y. Qiu, Y. Zhu, S. Hao, H. Zheng, Y. Li, Hydrogenolysis of glycerol to 1,3-propanediol over bifunctional catalysts containing Pt and heteropolyacids, Catal. Today, 212 (2013) 120-126.

[44] R. Arundhathi, T. Mizugaki, T. Mitsudome, K. Jitsukawa, K. Kaneda, Highly Selective Hydrogenolysis of Glycerol to 1,3-Propanediol over a Boehmite-Supported Platinum/Tungsten Catalyst, ChemSusChem, 6 (2013) $1345-1347$.

[45] R. Rodrigues, N. Isoda, M. Goncalves, F.C.A. Figueiredo, D. Mandelli, W.A. Carvalho, Effect of niobia and alumina as support for Pt catalysts in the hydrogenolysis of glycerol, Chem. Eng. J. (Amsterdam, Neth.), 198-199 (2012) 457-467.

[46] N. Ueda, Y. Nakagawa, K. Tomishige, Conversion of glycerol to ethylene glycol over Pt-modified Ni catalyst, Chem. Lett., 39 (2010) 506-507.

[47] Y. Li, H. Liu, L. Ma, D. He, Glycerol hydrogenolysis to propanediols over supported Pd-Re catalysts, RSC Adv., 4 (2014) 5503-5512.

[48] J. Ge, Z. Zeng, F. Liao, W. Zheng, X. Hong, S.C.E. Tsang, Palladium on iron oxide nanoparticles: the morphological effect of the support in glycerol hydrogenolysis, Green Chem., 15 (2013) 2064-2069.

[49] M.G. Musolino, L.A. Scarpino, F. Mauriello, R. Pietropaolo, Glycerol Hydrogenolysis Promoted by Supported Palladium Catalysts, ChemSusChem, 4 (2011) 1143-1150.

[50] M.G. Musolino, L.A. Scarpino, F. Mauriello, R. Pietropaolo, Selective transfer hydrogenolysis of glycerol 
promoted by palladium catalysts in absence of hydrogen, Green Chem., 11 (2009) 1511-1513.

[51] S. Koso, I. Furikado, A. Shimao, T. Miyazawa, K. Kunimori, K. Tomishige, Chemoselective hydrogenolysis of tetrahydrofurfuryl alcohol to 1,5-pentanediol, Chem. Commun. (Cambridge, U. K.), (2009) 2035-2037.

[52] S. Koso, N. Ueda, Y. Shinmi, K. Okumura, T. Kizuka, K. Tomishige, Promoting effect of Mo on the hydrogenolysis of tetrahydrofurfuryl alcohol to 1,5-pentanediol over Rh/SiO2, J. Catal., 267 (2009) 89-92.

[53] A. Shimao, S. Koso, N. Ueda, Y. Shinmi, I. Furikado, K. Tomishige, Promoting effect of Re addition to $\mathrm{Rh} / \mathrm{SiO} 2$ on glycerol hydrogenolysis, Chem. Lett., 38 (2009) 540-541.

[54] Y. Amada, S. Koso, Y. Nakagawa, K. Tomishige, Hydrogenolysis of 1,2-Propanediol for the Production of Biopropanols from Glycerol, ChemSusChem, 3 (2010) 728-736.

[55] K. Chen, S. Koso, T. Kubota, Y. Nakagawa, K. Tomishige, Chemoselective Hydrogenolysis of Tetrahydropyran-2-methanol to 1,6-Hexanediol over Rhenium-Modified Carbon-Supported Rhodium Catalysts, ChemCatChem, 2 (2010) 547-555.

[56] Y. Nakagawa, Y. Shinmi, S. Koso, K. Tomishige, Direct hydrogenolysis of glycerol into 1,3-propanediol over rhenium-modified iridium catalyst, J. Catal., 272 (2010) 191-194.

[57] Y. Shinmi, S. Koso, T. Kubota, Y. Nakagawa, K. Tomishige, Modification of $\mathrm{Rh} / \mathrm{SiO} 2$ catalyst for the hydrogenolysis of glycerol in water, Appl. Catal., B, 94 (2010) 318-326.

[58] Y. Amada, Y. Shinmi, S. Koso, T. Kubota, Y. Nakagawa, K. Tomishige, Reaction mechanism of the glycerol hydrogenolysis to 1,3-propanediol over Ir-ReOx/SiO2 catalyst, Appl. Catal., B, 105 (2011) 117-127.

[59] Y. Nakagawa, K. Tomishige, Catalyst Development for the Hydrogenolysis of Biomass-Derived Chemicals to Value-Added Ones, Catal. Surv. Asia, 15 (2011) 111-116.

[60] Y. Nakagawa, K. Tomishige, Heterogeneous catalysis of the glycerol hydrogenolysis, Catal. Sci. Technol., 1 (2011) 179-190.

[61] Y. Amada, H. Watanabe, Y. Hirai, Y. Kajikawa, Y. Nakagawa, K. Tomishige, Production of Biobutanediols by the Hydrogenolysis of Erythritol, ChemSusChem, 5 (2012) 1991-1999, S1991/1991-S1991/1910.

[62] Y. Amada, H. Watanabe, M. Tamura, Y. Nakagawa, K. Okumura, K. Tomishige, Structure of ReOx Clusters Attached on the Ir Metal Surface in Ir-ReOx/SiO2 for the Hydrogenolysis Reaction, J. Phys. Chem. C, 116 (2012) 23503-23514.

[63] K. Chen, K. Mori, H. Watanabe, Y. Nakagawa, K. Tomishige, C-O bond hydrogenolysis of cyclic ethers with OH groups over rhenium-modified supported iridium catalysts, J. Catal., 294 (2012) 171-183.

[64] S. Koso, H. Watanabe, K. Okumura, Y. Nakagawa, K. Tomishige, Stable Low-Valence ReOx Cluster Attached on Rh Metal Particles Formed by Hydrogen Reduction and Its Formation Mechanism, J. Phys. Chem. C, 116 (2012) 3079-3090.

[65] S. Koso, H. Watanabe, K. Okumura, Y. Nakagawa, K. Tomishige, Comparative study of Rh-MoOx and RhReOx supported on $\mathrm{SiO} 2$ for the hydrogenolysis of ethers and polyols, Appl. Catal., B, 111-112 (2012) 27-37.

[66] Y. Nakagawa, X. Ning, Y. Amada, K. Tomishige, Solid acid co-catalyst for the hydrogenolysis of glycerol to 1,3-propanediol over Ir-ReOx/SiO2, Appl. Catal., A, 433-434 (2012) 128-134.

[67] Y. Nakagawa, K. Tomishige, Production of 1,5-pentanediol from biomass via furfural and tetrahydrofurfuryl alcohol, Catal. Today, 195 (2012) 136-143.

[68] Y. Takeda, Y. Nakagawa, K. Tomishige, Selective hydrogenation of higher saturated carboxylic acids to alcohols using a $\mathrm{ReOx}-\mathrm{Pd} / \mathrm{SiO} 2$ catalyst, Catal. Sci. Technol., 2 (2012) 2221-2223.

[69] Y. Nakagawa, K. Mori, K. Chen, Y. Amada, M. Tamura, K. Tomishige, Hydrogenolysis of CO bond over Remodified Ir catalyst in alkane solvent, Appl. Catal., A, 468 (2013) 418-425.

[70] M. Tamura, Y. Amada, S. Liu, Z. Yuan, Y. Nakagawa, K. Tomishige, Promoting effect of Ru on Ir-ReOx/SiO2 catalyst in hydrogenolysis of glycerol, J. Mol. Catal. A: Chem., (2013) Ahead of Print.

[71] S. Liu, Y. Amada, M. Tamura, Y. Nakagawa, K. Tomishige, One-pot selective conversion of furfural into 1,5pentanediol over a Pd-added Ir-ReOx/SiO2 bifunctional catalyst, Green Chem., 16 (2014) 617-626.

[72] M. Chia, Y.J. Pagan-Torres, D. Hibbitts, Q.-H. Tan, H.N. Pham, A.K. Datye, M. Neurock, R.J. Davis, J.A. Dumesic, Selective Hydrogenolysis of Polyols and Cyclic Ethers over Bifunctional Surface Sites on RhodiumRhenium Catalysts, J. Am. Chem. Soc., 133 (2011) 12675-12689.

[73] M. Chia, B.J. O'Neill, R. Alamillo, P.J. Dietrich, F.H. Ribeiro, J.T. Miller, J.A. Dumesic, Bimetallic RhRe/C catalysts for the production of biomass-derived chemicals, J. Catal., 308 (2013) 226-236.

[74] T. Buntara, I. Melian-Cabrera, Q.H. Tan, J.L.G. Fierro, M. Neurock, J.G. de Vries, H.J. Heeres, Catalyst studies on the ring opening of tetrahydrofuran-dimethanol to 1,2,6-hexanetriol, Catal Today, 210 (2013) 106-116.

[75] K. Chen, M. Tamura, Z. Yuan, Y. Nakagawa, K. Tomishige, One-Pot Conversion of Sugar and Sugar Polyols to n-Alkanes without C-C Dissociation over the Ir-ReOx/SiO2 Catalyst Combined with H-ZSM-5, ChemSusChem, 6 (2013) 548 . 
[76] Y. Amada, H. Watanabe, Y. Hirai, Y. Kajikawa, Y. Nakagawa, K. Tomishige, Production of Biobutanediols by the Hydrogenolysis of Erythritol, Chemsuschem, 5 (2012) 1991-1999.

[77] J. Blumberger, B. Ensing, M.L. Klein, Formamide hydrolysis in alkaline aqueous solution: Insight from ab initio metadynamics calculations, Angew Chem Int Edit, 45 (2006) 2893-2897.

[78] M. Galib, G. Hanna, Mechanistic Insights into the Dissociation and Decomposition of Carbonic Acid in Water via the Hydroxide Route: An Ab Initio Metadynamics Study, J Phys Chem B, 115 (2011) 15024-15035.

[79] J.G. Lee, E. Asciutto, V. Babin, C. Sagui, T. Darden, C. Roland, Deprotonation of solvated formic acid: CarParrinello and metadynamics simulations, J Phys Chem B, 110 (2006) 2325-2331.

[80] E. Cauet, S.A. Bogatko, E.J. Bylaska, J.H. Weare, Ion Association in $\mathrm{AlCl} 3$ Aqueous Solutions from Constrained First-Principles Molecular Dynamics, Inorg Chem, 51 (2012) 10856-10869.

[81] N.L. Doltsinis, M. Sprik, Theoretical pK(a) estimates for solvated $\mathrm{P}(\mathrm{OH})(5)$ from coordination constrained CarParrinello molecular dynamics, Phys Chem Chem Phys, 5 (2003) 2612-2618.

[82] T. Ikeda, M. Hirata, T. Kimura, Hydrolysis of Al3 + from constrained molecular dynamics, J Chem Phys, 124 (2006).

[83] I. Ivanov, M.L. Klein, Deprotonation of a histidine residue in aqueous solution using constrained ab initio molecular dynamics, J Am Chem Soc, 124 (2002) 13380-13381.

[84] Q. Tan, First Principle Analysis of the Selective Ring Opening of Tetrahydrofurfural Alcohol over Rh Surface, Master's Thesis, University of Virginia, (2012).

[85] O.M. Daniel, A. DeLaRiva, E.L. Kunkes, A.K. Datye, J.A. Dumesic, R.J. Davis, X-ray Absorption Spectroscopy of Bimetallic Pt-Re Catalysts for Hydrogenolysis of Glycerol to Propanediols, Chemcatchem, 2 (2010) 1107-1114.

[86] J.D. Unruh, D. Pearson, Kirk-Othmer Encyclopedia of Chemical Technology, John Wiley \& Sons, NY, (2000).

[87] J.E. Logsdon, R.A. Loke, Kirk-Othmer Encyclopedia of Chemical Technology, John Wiley \& Sons, NY, (2000).

[88] M. Mavrikakis, M.A. Barteau, Oxygenate reaction pathways on transition metal surfaces, Journal of Molecular Catalysis A: Chemical, 131 (1998) 135-147.

[89] G. Kresse, J. Furthmüller, Efficiency of ab-initio total energy calculations for metals and semiconductors using a plane-wave basis set, Computational Materials Science, 6 (1996) 15-50.

[90] G. Kresse, J. Furthmüller, Efficient iterative schemes for ab initio total-energy calculations using a plane-wave basis set, Physical Review B, 54 (1996) 11169-11186.

[91] G. Kresse, J. Hafner, Ab initio molecular dynamics for liquid metals, Physical Review B, 47 (1993) 558-561.

[92] G. Kresse, J. Hafner, Ab initio molecular-dynamics simulation of the liquid-metal-amorphous-semiconductor transition in germanium, Physical Review B, 49 (1994) 14251-14269.

[93] J.P. Perdew, J.A. Chevary, S.H. Vosko, K.A. Jackson, M.R. Pederson, D.J. Singh, C. Fiolhais, Atoms, molecules, solids, and surfaces: Applications of the generalized gradient approximation for exchange and correlation, Physical Review B, 46 (1992) 6671-6687.

[94] P.E. Blöchl, Projector augmented-wave method, Physical Review B, 50 (1994) 17953-17979.

[95] G. Kresse, D. Joubert, From ultrasoft pseudopotentials to the projector augmented-wave method, Physical Review B, 59 (1999) 1758-1775.

[96] H.J. Monkhorst, J.D. Pack, Special points for Brillouin-zone integrations, Physical Review B, 13 (1976) 51885192.

[97] G. Henkelman, H. Jónsson, Improved tangent estimate in the nudged elastic band method for finding minimum energy paths and saddle points, The Journal of Chemical Physics, 113 (2000) 9978-9985.

[98] G. Henkelman, H. Jónsson, A dimer method for finding saddle points on high dimensional potential surfaces using only first derivatives, The Journal of Chemical Physics, 111 (1999) 7010-7022.

[99] W.C. Lu, C.Z. Wang, T.L. Chan, K. Ruedenberg, K.M. Ho, Representation of electronic structures in crystals in terms of highly localized quasiatomic minimal basis orbitals, Physical Review B, 70 (2004) 041101.

[100] W.C. Lu, C.Z. Wang, M.W. Schmidt, L. Bytautas, K.M. Ho, K. Ruedenberg, Molecule intrinsic minimal basis sets. I. Exact resolution of ab initio optimized molecular orbitals in terms of deformed atomic minimal-basis orbitals, The Journal of Chemical Physics, 120 (2004) 2629-2637.

[101] T.L. Chan, Y.X. Yao, C.Z. Wang, W.C. Lu, J. Li, X.F. Qian, S. Yip, K.M. Ho, Highly localized quasiatomic minimal basis orbitals for Mo from ab initio calculations, Physical Review B, 76 (2007) 205119.

[102] X. Qian, J. Li, L. Qi, C.-Z. Wang, T.-L. Chan, Y.-X. Yao, K.-M. Ho, S. Yip, Quasiatomic orbitals for ab initio tight-binding analysis, Physical Review B, 78 (2008) 245112.

[103] P.-O. Löwdin, On the Nonorthogonality Problem, in: L. Per-Olov (Ed.) Advances in Quantum Chemistry, Academic Press, 1970, pp. 185-199.

[104] P.O. Löwdin, On the Non - Orthogonality Problem Connected with the Use of Atomic Wave Functions in the 
Theory of Molecules and Crystals, The Journal of Chemical Physics, 18 (1950) 365-375.

[105] L.D. Kieken, M. Neurock, D.H. Mei, Screening by kinetic Monte Carlo simulation of Pt-Au(100) surfaces for the steady-state decomposition of nitric oxide in excess dioxygen, J Phys Chem B, 109 (2005) 2234-2244.

[106] D. Mei, P.A. Sheth, M. Neurock, C.M. Smith, First-principles-based kinetic Monte Carlo simulation of the selective hydrogenation of acetylene over Pd(111), J Catal, 242 (2006) 1-15.

[107] E.W. Hansen, M. Neurock, First-principles-based Monte Carlo simulation of ethylene hydrogenation kinetics on Pd, J Catal, 196 (2000) 241-252.

[108] E. Hansen, M. Neurock, First-principles based kinetic simulations of acetic acid temperature programmed reaction on Pd(111), J Phys Chem B, 105 (2001) 9218-9229.

[109] M. Neurock, D.H. Mei, Effects of alloying Pd and Au on the hydrogenation of ethylene: An ab initio-based dynamic Monte Carlo study, Top Catal, 20 (2002) 5-23.

[110] J.E. Rekoske, R.D. Cortright, S.A. Goddard, S.B. Sharma, J.A. Dumesic, Microkinetic Analysis of Diverse Experimental-Data for Ethylene Hydrogenation on Platinum, J Phys Chem-Us, 96 (1992) 1880-1888.

[111] K. Tomishige, S. Kanazawa, M. Sato, K. Ikushima, K. Kunimori, Catalyst design of pt-modified Ni/Al2O3 catalyst with flat temperature profile in methane reforming with CO2 and O-2, Catal Lett, 84 (2002) 69-74.

[112] V.V. Ordomsky, J. van der Schaaf, J.C. Schouten, T.A. Nijhuis, The effect of solvent addition on fructose dehydration to 5-hydroxymethylfurfural in biphasic system over zeolites, J Catal, 287 (2012) 68-75.

[113] V.V. Ordomsky, J. van der Schaaf, J.C. Schouten, T.A. Nijhuis, Fructose Dehydration to 5Hydroxymethylfurfural over Solid Acid Catalysts in a Biphasic System, Chemsuschem, 5 (2012) 1812-1819.

[114] W.G. Hoover, Canonical dynamics: Equilibrium phase-space distributions, Physical Review A, 31 (1985) 1695-1697.

[115] S. Nose, A unified formulation of the constant-temperature molecular-dynamics methods, J. Chem. Phys., 81 (1984) 511-519.

[116] L. Zhang, A.M. Karim, M.H. Engelhard, Z.H. Wei, D.L. King, Y. Wang, Correlation of Pt-Re surface properties with reaction pathways for the aqueous-phase reforming of glycerol, J Catal, 287 (2012) 37-43.

[117] K.G. Azzam, I.V. Babich, K. Seshan, B.L. Mojet, L. Lefferts, Stable and Efficient Pt-Re/TiO2 catalysts for Water-Gas-Shift: On the Effect of Rhenium, Chemcatchem, 5 (2013) 557-564.

[118] M.J. Janik, J. Macht, E. Iglesia, M. Neurock, Correlating Acid Properties and Catalytic Function: A FirstPrinciples Analysis of Alcohol Dehydration Pathways on Polyoxometalates, J Phys Chem C, 113 (2009) 1872-1885.

[119] J. Macht, M.J. Janik, M. Neurock, E. Iglesia, Mechanistic consequences of composition in acid catalysis by polyoxometalate Keggin clusters, J Am Chem Soc, 130 (2008) 10369-10379.

[120] M.J. Janik, R.J. Davis, M. Neurock, The relationship between adsorption and solid acidity of heteropolyacids, Catal Today, 105 (2005) 134-143.

[121] S. Hammes-Schiffer, Comparison of hydride, hydrogen atom, and proton-coupled electron transfer reactions, Chemphyschem, 3 (2002) 33-42.

[122] G.J. Martyna, M.E. Tuckerman, Symplectic Reversible Integrators - Predictor Corrector Methods, J Chem Phys, 102 (1995) 8071-8077.

[123] D. Marx, M.E. Tuckerman, J. Hutter, M. Parrinello, The nature of the hydrated excess proton in water, Nature, 397 (1999) 601-604.

[124] N.S. Chang, S. Aldrett, M.T. Holtzapple, R.R. Davison, Kinetic studies of ketone hydrogenation over Raney nickel catalyst, Chem Eng Sci, 55 (2000) 5721-5732.

[125] U.K. Singh, M.A. Vannice, Kinetics of liquid-phase hydrogenation reactions over supported metal catalysts - a review, Appl Catal a-Gen, 213 (2001) 1-24.

[126] N.K. Sinha, M. Neurock, A first principles analysis of the hydrogenation of C-1-C-4 aldehydes and ketones over Ru(0001), J Catal, 295 (2012) 31-44.

[127] B.S. Akpa, C. D'Agostino, L.F. Gladden, K. Hindle, H. Manyar, J. McGregor, R. Li, M. Neurock, N. Sinha, E.H. Stitt, D. Weber, J.A. Zeitler, D.W. Rooney, Solvent effects in the hydrogenation of 2-butanone, J Catal, 289 (2012) 30-41.

[128] S.K. Desai, M. Neurock, First-principles study of the role of solvent in the dissociation of water over a Pt-Ru alloy, Phys Rev B, 68 (2003).

[129] D. Hibbitts, Q. Tan, M. Neurock, Acidity of Hydroxides on Alloys of Noble Metals and Oxophilic Oxide Promoters such as Rh-ReOx, J Catal, (to be published) (2014).

[130] S.F. Lincoln, D.T. Richens, A.G. Sykes, 1.25 - Metal Aqua Ions, in: J.A. McCleverty, T.J. Meyer (Eds.) Comprehensive Coordination Chemistry II, Pergamon, Oxford, 2003, pp. 515-555.

[131] L.A. Friedman, F. You, M. Sabat, W.D. Harman, Rhenium-Promoted Diastereo- and Enantioselective Cyclopentannulation Reactions: Furans as 1,3-Propene Dipoles, J. Am. Chem. Soc., 125 (2003) 14980-14981. 
[132] Y. Jiang, J. Hess, T. Fox, H. Berke, Rhenium Hydride/Boron Lewis Acid Cocatalysis of Alkene Hydrogenations: Activities Comparable to Those of Precious Metal Systems, J. Am. Chem. Soc., 132 (2010) 1823318247.

[133] Y. Jiang, W. Huang, H.W. Schmalle, O. Blacque, T. Fox, H. Berke, Efficient Lewis Acid Promoted Alkene Hydrogenations Using Dinitrosyl Rhenium(-I) Hydride Catalysts, Organometallics, 32 (2013) 7043-7052.

[134] S. Nagashima, S. Furukawa, S. Kamiguchi, R. Kajio, H. Nagashima, A. Yamaguchi, M. Shirai, H. Kurokawa, T. Chihara, Catalytic Activity of Molecular Rhenium Sulfide Clusters [Re6S8(OH)6-n(H2O)n](4-n)- $(\mathrm{n}=0,2,4,6)$ with Retention of the Octahedral Metal Frameworks: Dehydrogenation and Dehydration of 1,4-Butanediol, J. Cluster Sci., (2014) Ahead of Print.

[135] L.L. Ouh, T.E. Mueller, Y.K. Yan, Intramolecular hydroamination of 6-aminohex-1-yne catalyzed by Lewis acidic rhenium(I) carbonyl complexes, J. Organomet. Chem., 690 (2005) 3774-3782.

[136] G.A. Stark, J.A. Gladysz, Additions of carbon nucleophiles to acyclic imine complexes of the chiral rhenium Lewis acid $[(\eta 5-\mathrm{C} 5 \mathrm{H} 5) \operatorname{Re}(\mathrm{NO})(\mathrm{PPh} 3)]+$ : controlling factors in 1,3-asymmetric induction and syntheses of nonracemic organic amines, Inorg. Chim. Acta, 269 (1998) 167-180.

[137] F. You, L.A. Friedman, K.C. Bassett, Y. Lin, M. Sabat, W.D. Harman, Rhenium(I) ๆ2-Coordinated Furan Complexes: Converting Furan into a 1,3-Carbon Dipole, Organometallics, 24 (2005) 2903-2912.

[138] E.L. Kunkes, D.A. Simonetti, J.A. Dumesic, W.D. Pyrz, L.E. Murillo, J.G. Chen, D.J. Buttrey, The role of rhenium in the conversion of glycerol to synthesis gas over carbon supported platinum-rhenium catalysts, J. Catal., 260 (2008) 164-177.

[139] E.L. Kunkes, R.R. Soares, D.A. Simonetti, J.A. Dumesic, An integrated catalytic approach for the production of hydrogen by glycerol reforming coupled with water-gas shift, Appl. Catal., B, 90 (2009) 693-698.

[140] P.J. Dietrich, R.J. Lobo-Lapidus, T. Wu, A. Sumer, M.C. Akatay, B.R. Fingland, N. Guo, J.A. Dumesic, C.L. Marshall, E. Stach, J. Jellinek, W.N. Delgass, F.H. Ribeiro, J.T. Miller, Aqueous Phase Glycerol Reforming by PtMo Bimetallic Nano-Particle Catalyst: Product Selectivity and Structural Characterization, Top. Catal., 55 (2012) 53-69.

[141] P.J. Dietrich, T. Wu, A. Sumer, J.A. Dumesic, J. Jellinek, W.N. Delgass, F.H. Ribeiro, J.T. Miller, Aqueous Phase Glycerol Reforming with Pt and PtMo Bimetallic Nanoparticle Catalysts: The Role of the Mo Promoter, Top. Catal., 56 (2013) 1814-1828.

[142] H.G. Manyar, C. Paun, R. Pilus, D.W. Rooney, J.M. Thompson, C. Hardacre, Highly selective and efficient hydrogenation of carboxylic acids to alcohols using titania supported Pt catalysts, Chem Commun, 46 (2010) 62796281 .

[143] D.J. Braden, C.A. Henao, J. Heltzel, C.C. Maravelias, J.A. Dumesic, Production of liquid hydrocarbon fuels by catalytic conversion of biomass-derived levulinic acid, Green Chem., 13 (2011) 1755-1765.

[144] M. Brandle, J. Sauer, Acidity differences between inorganic solids induced by their framework structure. A combined quantum mechanics molecular mechanics ab initio study on zeolites, J Am Chem Soc, 120 (1998) 15561570 .

[145] Y. Sawama, Y. Ogata, K. Kawamoto, H. Satake, K. Shibata, Y. Monguchi, H. Sajiki, Y. Kita, Lewis AcidCatalyzed Ring-Opening Functionalizations of 1,4-Epoxy-1,4-dihydronaphthalenes, Adv Synth Catal, 355 (2013) 517-528.

[146] P.J. Linstrom, W.G. Mallard, NIST Chemistry WebBook, NIST Standard Reference Database Number 69, National Institute of Standards and Technology, Gaithersburg MD, 20899, http://webbook.nist.gov.

[147] A. Jentys, G. Warecka, M. Derewinski, J.A. Lercher, Adsorption of Water on Zsm5 Zeolites, J Phys Chem-Us, 93 (1989) 4837-4843.

[148] G.M. Brown, M.R. Noe-Spirlet, W.R. Busing, H.A. Levy, Dodecatungstophosphoric acid hexahydrate, $\left(\mathrm{H}_{5} \mathrm{O}_{2}{ }^{+}\right)_{3}\left(\mathrm{PW}_{12} \mathrm{O}_{40}{ }^{3-}\right)$. The true structure of Keggin's 'pentahydrate' from single-crystal X-ray and neutron diffraction data, Acta Crystallographica Section B, 33 (1977) 1038-1046.

[149] J.K. Norskov, T. Bligaard, A. Logadottir, J.R. Kitchin, J.G. Chen, S. Pandelov, J.K. Norskov, Trends in the exchange current for hydrogen evolution, J Electrochem Soc, 152 (2005) J23-J26.

[150] T. Bucko, L. Benco, J. Hafner, J.G. Angyan, Proton exchange of small hydrocarbons over acidic chabazite: Ab initio study of entropic effects, J Catal, 250 (2007) 171-183.

[151] J.P. Ryckaert, G. Ciccotti, H.J.C. Berendsen, Numerical-Integration of Cartesian Equations of Motion of a System with Constraints - Molecular-Dynamics of N-Alkanes, J Comput Phys, 23 (1977) 327-341.

[152] E.A. Carter, G. Ciccotti, J.T. Hynes, R. Kapral, Constrained Reaction Coordinate Dynamics for the Simulation of Rare Events, Chem Phys Lett, 156 (1989) 472-477.

[153] E. Darve, M.A. Wilson, A. Pohorille, Calculating free energies using a scaled-force molecular dynamics algorithm, Mol Simulat, 28 (2002) 113-144.

[154] W.K. Den Otter, W.J. Briels, Free energy from molecular dynamics with multiple constraints, Mol Phys, 98 
(2000) 773-781.

[155] P. Fleurat-Lessard, T. Ziegler, Tracing the minimum-energy path on the free-energy surface, J Chem Phys, 123 (2005).

[156] P. Atkens, Atkins' Physical Chemistry (8th edn W. H. Freeman), (2006).

[157] R.N. Goldberg, N. Kishore, R.M. Lennen, Thermodynamic Quantities for the Ionization Reactions of Buffers, Journal of Physical and Chemical Reference Data, 31 (2002) 231-370.

[158] W.D. Kumler, J.J. Eiler, The Acid Strength of Mono and Diesters of Phosphoric Acid. The n-Alkyl Esters from Methyl to Butyl, the Esters of Biological Importance, and the Natural Guanidine Phosphoric Acids, J Am Chem Soc, 65 (1943) 2355-2361.

[159] I.M. Kolthoff, P.J. EIving, Treatise on Analytical Chemistry, The Interscience Encyclopedia Inc., New York, (1959).

[160] J.-S. Filhol, M. Neurock, Elucidation of the Electrochemical Activation of Water over Pd by First Principles, Angewandte Chemie International Edition, 45 (2006) 402-406.

[161] A. Banerjee, J.O. Jensen, J. Simons, Translation-Rotation Invariance for N-Particle Systems - Internal Coordinates and Search for Stationary-Points in Reduced Spaces, J Chem Phys, 83 (1985) 3500-3506. 